Draft VERSion November 6, 2018

Preprint typeset using $\mathrm{L}^{A} \mathrm{~T}_{\mathrm{E}} \mathrm{X}$ style emulateapj v. 11/10/09

\title{
THE DISK POPULATION OF THE TAURUS STAR-FORMING REGION ${ }^{1}$
}

\author{
K. L. Luhman ${ }^{2,3}$, P. R. Allen $^{2}$, C. Espaillat ${ }^{4,5}$, L. Hartmann ${ }^{4}$, and N. Calvet ${ }^{4}$ \\ Draft version November 6, 2018
}

\begin{abstract}
We have analyzed nearly all images of the Taurus star-forming region at 3.6, 4.5, 5.8, 8.0, and $24 \mu \mathrm{m}$ that were obtained during the cryogenic mission of the Spitzer Space Telescope $\left(46 \mathrm{deg}^{2}\right)$ and have measured photometry for all known members of the region that are within these data, corresponding to 348 sources, or $99 \%$ of the known stellar population. By combining these measurements with previous observations with the Spitzer Infrared Spectrograph and other facilities, we have classified the members of Taurus according to whether they show evidence of circumstellar disks and envelopes (classes I, II, and III). Through these classifications, we find that the disk fraction in Taurus, N(II)/N(II+III), is $\sim 75 \%$ for solar-mass stars and declines to $\sim 45 \%$ for low-mass stars and brown dwarfs $(0.01-$ $\left.0.3 M_{\odot}\right)$. This dependence on stellar mass is similar to that measured for Chamaeleon I, although the disk fraction in Taurus is slightly higher overall, probably because of its younger age (1 Myr vs. 2-3 Myr). In comparison, the disk fraction for solar-mass stars is much lower $(\sim 20 \%)$ in IC 348 and $\sigma$ Ori, which are denser than Taurus and Chamaeleon I and are roughly coeval with the latter. These data indicate that disk lifetimes for solar-mass stars are longer in star-forming regions that have lower stellar densities. Through an analysis of multiple epochs of Spitzer photometry that are available for $\sim 200$ Taurus members, we find that stars with disks exhibit significantly greater mid-infrared variability than diskless stars, which agrees with the results of similar variability measurements for a smaller sample of stars in Chamaeleon I. The variability fraction for stars with disks is higher in Taurus than in Chamaeleon I, indicating that the IR variability of disks decreases with age. Finally, we have used our data in Taurus to refine the observational criteria for primordial, evolved, and transitional disks. The ratio of the number of evolved and transitional disks to the number of primordial disks in Taurus is 15/98 for spectral types of K5-M5, indicating a timescale of $0.15 \times \tau_{\text {primordial }} \sim 0.45 \mathrm{Myr}$ for the clearing of the inner regions of optically thick disks. After applying the same criteria to older clusters and associations (2-10 Myr) that have been observed with Spitzer, we find that the proportions of evolved and transitional disks in those populations are consistent with the measurements in Taurus when their star formation histories are properly taken into account.
\end{abstract}

ERRATUM: In Table 7, we inadvertently omitted the spectral type bins in which class II sources were placed in Table 8 based on their bolometric luminosities (applies only to stars that lack spectroscopic classifications). The bins were K6-M3.5 for FT Tau, DK Tau B, and IRAS 04370+2559, M3.5-M6 for IRAS 04200+2759, IT Tau B, and ITG 1, and M6-M8 for IRAS 04325+2402 C. In addition, the values of $K_{s}-$ [3.6] in Table 13 and Figure 26 for spectral types of M4-M9 are incorrect. We present corrected versions of Table 13 and Figure 26.

Subject headings: accretion disks — planetary systems: protoplanetary disks — stars: formation — stars: low-mass, brown dwarfs — stars: pre-main sequence

\section{INTRODUCTION}

Much of our knowledge of the processes of star and planet formation has been derived from observations of circumstellar disks around newborn stars. The Taurus complex of dark clouds is one of the principle sites for studies of disks. Taurus is among the nearest starforming regions to the $\operatorname{Sun}(d=140 \mathrm{pc})$ and contains more than 300 young stars and brown dwarfs (Kenyon et al. 2008). Because of the low stellar den-

\footnotetext{
${ }^{1}$ Based on observations performed with the Spitzer Space Telescope.

2 Department of Astronomy and Astrophysics, The Pennsylvania State University, University Park, PA 16802; kluhman@astro.psu.edu.

${ }^{3}$ Center for Exoplanets and Habitable Worlds, The Pennsylvania State University, University Park, PA 16802.

${ }^{4}$ Department of Astronomy, The University of Michigan, Ann Arbor, MI 48109.

${ }^{5}$ Current address: Harvard-Smithsonian Center for Astrophysics, Cambridge, MA 02138.
}

sity and the absence of photoionizing stars, most members of Taurus are not embedded within bright nebulae, and thus are not subject to high levels of background emission that would hinder infrared (IR) observations of disks. The low density of Taurus also permitted resolved photometry of individual stars with the low-resolution imaging that was offered by the first major far-IR telescope, the Infrared Astronomical Satellite (IRAS, Beichman et al. 1986; Kenvon et al. 1990, 1994). By combining 12-100 $\mu \mathrm{m}$ photometry from IRAS with 1-10 $\mu \mathrm{m}$ data from ground-based telescopes, Kenyon \& Hartmann (1995) performed the most comprehensive census of disks in a star-forming region at that time. The disk-bearing stars identified in Taurus have served as targets for a multitude of detailed observations of circumstellar disks (Kenvon et al. 2008, references therein).

The census of the stellar population of Taurus has expanded significantly since the disk study of 
Kenyon \& Hartmann (1995), particularly at masses below $0.5 M_{\odot}$. The sensitivities of IR telescopes also have improved dramatically, most notably with the deployment of the Spitzer Space Telescope (Werner et al. 2004), which is capable of detecting members of Taurus with masses below $0.01 M_{\odot}$. The Taurus stellar population has been imaged extensively by Spitzer through pointed observations of individual stars as well as widefield maps. These images have been used to measure photometry for known members of Taurus (Hartmann et al. 2005; Luhman et al. 2006; Guieu et al. 2007) and search for new disk-bearing members (Luhman et al. 2006, 2009a, b; Rebull et al. 2010).

With the recent completion of the cryogenic mission of Spitzer, we wish to present a study of disks in Taurus that makes use of all Spitzer images of the region, which cover a total area of $46 \mathrm{deg}^{2}$ and encompass $99 \%$ of the known stellar population. We begin by analyzing all Spitzer images of Taurus at 3.6-24 $\mu \mathrm{m}$ that are publicly available and measuring photometry for all known members that are detected in these data $(\S[2)$. We use these measurements in conjunction with previous observations by the Spitzer Infrared Spectrograph and other telescopes to classify the spectral energy distributions of all members of Taurus ( $\S$ 3). Through these classifications, we investigate how the prevalence of disks depends on stellar mass $(\S 4)$ and location $(\S 5)$. We then characterize the mid-IR variability of disks in Taurus with the multi-epoch photometry from Spitzer (§ 6). Finally, we use our mid-IR data in Taurus to refine the observational criteria for the advanced stages of disk evolution and we apply these criteria to Spitzer measurements in nearby clusters and associations with ages of 2-10 Myr ( $(7)$.

\section{INFRARED IMAGES}

\subsection{Observations}

For our census of the disk population in Taurus, we use images at 3.6, 4.5, 5.8, and $8.0 \mu \mathrm{m}$ obtained with Spitzer's Infrared Array Camera (IRAC; Fazio et al. 2004) and images at $24 \mu \mathrm{m}$ obtained with the Multiband Imaging Photometer for Spitzer (MIPS; Rieke et al. 2004). The fields of view are $5 ! 2 \times 5 ! 2$ and $5 ! 4 \times 5{ }^{\prime} 4$ for IRAC and the $24 \mu \mathrm{m}$ channel of MIPS, respectively. The cameras produced images with FWHM=1".6-1".9 from 3.6 to $8.0 \mu \mathrm{m}$ and $\mathrm{FWHM}=55^{\prime \prime} 9$ at $24 \mu \mathrm{m}$.

We consider all IRAC and MIPS $24 \mu \mathrm{m}$ observations that have been performed in the vicinity of the Taurus clouds with the exception of some of the data with program identifications (PIDs) of 50477 and 50584, which are not yet available to the public. The images from the latter programs encompass only a small number of known members of Taurus, as discussed later in this section. The available data were obtained through Guaranteed Time Observations for $\mathrm{PID}=6,36,37$ (G. Fazio), 53 (G. Rieke), 94 (C. Lawrence), 30540 (G. Fazio, J. Houck), and 40302 (J. Houck), Director's Discretionary Time for $\mathrm{PID}=462$ (L. Rebull), Legacy programs for $\mathrm{PID}=139$, 173 (N. Evans), and 30816 (D. Padgett), and General Observer programs for PID=3584 (D. Padgett), 20302 (P. André), 20386 (P. Myers), 20762 (J. Swift), 30384 (T. Bourke), 40844 (C. McCabe), and 50584 (D. Padgett). The IRAC and MIPS observations were performed through 180 and 137 Astronomical Observation Requests
(AORs), respectively. The characteristics of the resulting images are summarized in Tables 1 and 2. All AORs in Taurus with publicly available data are included in these observing logs, even those that do not contain known members and thus do not contribute photometry to our disk census. The boundaries of the IRAC and MIPS images are indicated in maps of the Taurus dark clouds in Figures 1 and 2, respectively. Each camera observed a total area of $46 \mathrm{deg}^{2}$. The fields covered by the two cameras closely overlap, as demonstrated in Figures 11 and 2 .

We adopt the census of the stellar population in Taurus that was described by Luhman et al. (2009b), which is an updated version of the one presented by Kenyon et al. (2008). This census consists of 352 and 341 sources that are resolvable by IRAC and MIPS $24 \mu \mathrm{m}$ images, respectively, 346 and 299 of which are within the available IRAC and MIPS images. Two of the members in the MIPS images were not observed by IRAC. Thus, a total of 348 resolved members appear within images from either IRAC or MIPS, corresponding to $99 \%$ of the known stellar population. All known members of Taurus are included in the tabulations of photometry in the next section. The stars that are outside of all available images for a given camera are indicated in those tables. The IRAC and MIPS images that are not yet available to the public encompass three and four known members, respectively. One and three of these stars are outside of the currently available IRAC and MIPS images, which consist of XEST 06-006 for IRAC and XEST 06-006, 2MASS J04163048+3037053, and 2MASS J04162725+2053091 for MIPS.

\subsection{Data Reduction}

Initial processing of the IRAC and MIPS images was performed by the Spitzer Science Center (SSC) pipeline. The pipeline versions were S14.0.0, S14.4.0, S15.3.0, S16.1.0, and S18.7.0 for IRAC and S16.0.1, S16.1.0, S16.1.1, and S17.2.0 for MIPS. The IRAC images produced by the SSC pipeline were combined into mosaics using R. Gutermuth's WCSmosaic IDL package (Gutermuth et al. 2008). We selected a plate scale of 0 .'86 pixel $^{-1}$ for these final images. For MIPS, we used the mosaics produced by the SSC pipeline, which had a plate scale of 2 .' 45 pixel $^{-1}$.

We measured photometry for the known members of Taurus with methods similar to those employed in our previous Spitzer studies of star-forming regions (Luhman et al. 2008a b). We used the IRAF task STARFIND to measure the positions of the Taurus members in the IRAC and MIPS images and we performed aperture photometry at those locations using the IRAF task PHOT. For MIPS sources, the radii of the apertures and inner and outer boundaries of the sky annuli were 3 , 3 , and 4 pixels, respectively. For a given IRAC source, we selected one of the apertures listed in Table 3 . The 4 pixel apertures were the default choices. We visually inspected the IRAC images of all stars to determine if smaller apertures were needed because of close proximity to other sources. Based on this inspection, we adopted aperture radii of 3 pixels and sky annuli extending from 3 to 4 pixels for the IRAC measurements of JH 112 and 2MASS J04324938+2253082. For sources that were very close to other stars, we attempted to remove the 
light from the latter by subtracting a scaled IRAC point spread function (PSF, Marengo et al. 2006). We performed this step prior to the measurement of photometry for UZ Tau Ba+Bb, DK Tau B, IT Tau B, HV Tau C, MHO 1, MHO 2, Haro 6-37 A and B, V710 Tau A and B, J1-4872 A and B, IRAS 04191+1523 B, HD 28867 A and B, and FU Tau B (Luhman et al. 2009a). We measured photometry from each PSF-subtracted image with aperture radii and inner and outer sky annuli of either 2 , 14 , and 15 pixels or 3,3 , and 4 pixels, respectively. We selected the aperture from these two options that minimized contamination by the residuals of the neighboring PSF-subtracted star.

Many of the protostars in Taurus are surrounded by extended emission in the IRAC images. We measured photometry for these sources using both the default 4-pixel apertures as well as larger apertures that encompass most of the scattered light. L1521F-IRS and IRAS $04166+2706$ exhibit only extended emission and no point sources at $3.6-5.8$ and $3.6 \mu \mathrm{m}$, respectively. Therefore, we did not apply the 4-pixel apertures to these images and measured photometry from only the large apertures. Because IRAS $04325+2402 \mathrm{C}$ is faint compared to its surrounding emission, we estimated its IRAC photometry through PSF fitting.

To calibrate the photometry, we adopted zero point magnitudes $(Z P)$ of $19.670,18.921,16.855,17.394$, and 15.119 in the $3.6,4.5,5.8,8.0$, and $24 \mu \mathrm{m}$ bands, where $M=-2.5 \log (D N /$ sec $)+Z P$ (Reach et al. 2005; Engelbracht et al. 2007). For each AOR that contained stars that were isolated, bright, and unsaturated, we used these stars to measure aperture corrections between our adopted apertures and the larger ones employed by Reach et al. (2005) and Engelbracht et al. (2007). For the MIPS data, we combined our aperture corrections with the one estimated by Engelbracht et al. (2007) between their aperture and an infinite one. These aperture corrections were then applied to our photometry for all other sources in that AOR. If an AOR did not contain stars that were suitable for estimating aperture corrections, we adopted the averages of the corrections measured among all of the AORs. The average aperture corrections for IRAC are given in Table 3. The spread in these measurements was less than \pm 0.01 mag for a given band and aperture. The corrections to an infinite aperture for MIPS ranged between 0.78-0.84 mag with an average value of $0.80 \mathrm{mag}$.

We present the IRAC and MIPS photometry for the known members of Taurus in Tables 4, 5, and 6. If an object is saturated or is not detected in all MIPS images of that position, it appears only once in Table 6 and the observing dates are omitted. Our quoted photometric errors include the Poisson errors in the source and background emission and the $2 \%$ and $4 \%$ uncertainties in the calibrations of IRAC and MIPS, respectively (Reach et al. 2005; Engelbracht et al. 2007). The errors do not include an additional error of $\pm 0.05 \mathrm{mag}$ due to location-dependent variations in the IRAC calibration.

\section{CLASSIFICATIONS OF SPECTRAL ENERGY DISTRIBUTIONS}

\subsection{Known Class 0 and Class I Sources}

Spectral energy distributions (SEDs) of young stars are sensitive to the presence of circumstellar dust, and thus can be used to constrain the evolutionary stages of the members of a young stellar population. These stages consist of a protostar surrounded by an accretion disk and an infalling envelope (classes 0 and I), a star with a disk but no envelope (class II), and a star that is no longer surrounded by primordial dust (class III, Lada \& Wilking 1984; Lada 1987; André et al. 1993; Greene et al. 1994). We wish to classify the members of Taurus with SEDs constructed from our Spitzer photometry. However, while class III stars are easily distinguished from less evolved systems, some class I and class II sources can exhibit similar SEDs in the Spitzer bands (Hartmann et al. 2005). The definitive identification of class I sources requires other observations that better constrain the presence of an envelope, such as mid-IR spectroscopy, far-IR and millimeter photometry, and high-resolution images. Therefore, before classifying the Spitzer SEDs in Taurus, we assign classes 0 and I to all members of Taurus that exhibit evidence of envelopes in previous data. These stars consist of all targets from Watson et al. (2004) and Furlan et al. (2008)6, IRAS 04108+2803 A (Zasowski et al. 2009), L1551NE (Moriarty-Schieven et al. 1995), IRAS 04191+1523 A (Tamura et al. 1991; Moriarty-Schieven et al. 1992), IRAS 04191+1523 B (Motte 1998; Duchêne et al. 2004; Dunham et al. 2006), IRAM 04191+1522 (André et al. 1999; Dunham et al. 2006), L1521F-IRS (Bourke et al. 2006), IRAS 04111+2800G (Prusti et al. 1992), and IRAS 04166+2706 (Kenyon et al. 1990; Bontemps et al. 1996; Motte \& André 2001). Haro 6-5B also is a class I source according to unpublished data from the Spitzer Infrared Spectrograph (IRS, D. Watson, private communication). Among these protostars, IRAS 04368+2557 and IRAM $04191+1522$ are the clearest examples of class 0 sources based on their low bolometric temperatures $\left(T_{\text {bol }}<70 \mathrm{~K}\right.$, Chen et al. 1995; André et al. 1999; Motte \& André 2001). We adopt class I designations for the remaining stars. A few of these sources appear to be in transition between classes 0 and I (L1551NE, Chen et al. 1995) or between classes I and II (IRAS 04154+2823, Furlan et al. 2008). The latter stage has been referred to as flat-spectrum or class I-II in some studies, but we denote them as class I for the purposes of this work since they show evidence of envelopes (albeit tenuous ones).

The stars described in this section that exhibit evidence of envelopes in previous observations comprise all class 0 and I sources in Table 7 . Sources that have class I SEDs but lack supporting observations that are sensitive to the presence of envelopes are listed as "class I?" (\$3.3).

\subsection{Infrared Color-Color $\&$ Color-Magnitude Diagrams}

To provide an initial demonstration of how stars with disks are identified with Spitzer photometry, we can construct color-color and color-magnitude diagrams from our Spitzer data for Taurus. These diagrams are frequently applied to young stellar populations because they effectively discriminate between stars with and without

\footnotetext{
6 The IRS data from Furlan et al. (2008) that were attributed to IRAS $04166+2706$ actually apply to IRAS $04166+2708$. This misidentification of IRAS $04166+2706$ with the counterpart of IRAS $04166+2708$ also appears in Luhman (2006), Luhman et al. $(2006)$, and Kenyon et al. (2008), and probably originated in the former two papers.
} 
disks and yet they do not require knowledge of the individual stellar properties, such as spectral types and extinctions (Allen et al. 2004; Megeath et al. 2004).

If multiple measurements are available in a given band for a star, we adopt the mean of those measurements weighted by the inverse square of their flux errors in the color-color and color-magnitude diagrams. Some Taurus members lack photometry in one or more bands and thus are absent from a given diagram because they are unresolved from brighter sources, outside the field of view of the Spitzer images, saturated, detected only as extended emission, or below the detection limit (only applies to the $24 \mu \mathrm{m}$ data). If a binary is resolved by IRAC but not by MIPS, we plot the total system magnitudes when data from both cameras are used in a diagram.

In Figure 3, we present two IRAC color-color diagrams for the members of Taurus that have been measured in all of the IRAC bands. Some of the stars reside in a tight group near the origin while others form a broad distribution of redder sources, which correspond to stellar photospheres and stars with disks, respectively. As expected, the known class 0/I sources exhibit the reddest colors since they are surrounded by the both circumstellar disks and envelope, although a few protostars have relatively blue colors because they appear nearly edgeon from our point of view (e.g., IRAS 04302+2247).

In Figure 4, we plot the members of Taurus in a colorcolor diagram that combines IRAC and MIPS data and an IRAC color-magnitude diagram. As in the IRAC color-color diagrams, two distinct populations of diskless and disk-bearing stars are apparent in both diagrams. The IRAC/MIPS color-color diagram is useful for identifying stars that exhibit excess emission at $24 \mu \mathrm{m}$ but not in the IRAC bands, which is a signature of a disk with an inner hole. Disks of this kind are discussed in more detail in $\S 7$ Meanwhile, the color-magnitude diagram in Figure 4 illustrates the dependence of mid-IR colors on magnitude, which acts as a proxy for luminosity, spectral type, and stellar mass. At fainter magnitudes, the sequence of diskless stars becomes slightly redder, reflecting a variation of the photospheric colors with spectral type, while the average excess at $8 \mu \mathrm{m}$ of the sources with disks becomes smaller. The same trends have been found in previous IRAC measurements for Taurus (Luhman et al. 2006).

\subsection{Classification with Spectral Slopes}

Most members of Taurus are easily classified as either class III or class 0/I/II with Spitzer color-color diagrams. However, the color-color diagrams do not offer a straightforward means of dividing disk-bearing stars among classes 0, I, and II. In addition, some stars have ambiguous colors that are neither neutral nor very red, which can arise from diskless stars that are highly reddened or very cool, or stars that harbor disks in advanced stages of evolution. We can perform more refined and quantitative classifications by characterizing the SED of each star in terms of spectral slopes that are defined as $\alpha=d \log \left(\lambda F_{\lambda}\right) / d \log (\lambda)$ Adams et al. 1987; Lada 1987; Greene et al. 1994), correcting the slopes for reddening, and comparing the resulting values to the typical slopes of stellar photospheres near the spectral type in question.

As done in Chamaeleon I (Luhman et al. 2008a), we have computed slopes in Taurus between four pairs of bands, $K_{s} /[8.0], K_{s} /[24],[3.6] /[8.0]$, and $[3.6] /[24]$. For most stars, we use measurements of $K_{s}(2.2 \mu \mathrm{m})$ from the Point Source Catalog of the Two-Micron All-Sky Survey (2MASS, Skrutskie et al. 2006). We adopt photometry from multiplicity surveys for a few systems that are marginally resolved by $2 \mathrm{MASS}$. As in the colorcolor diagrams, we have used the weighted average in a given band if an object has been observed at multiple epochs. For binaries that are resolved in $K_{s}$ and the IRAC bands but not at $24 \mu \mathrm{m}$, we computed the $24 \mu \mathrm{m}$ slopes with the total system fluxes at shorter wavelengths. These systems are noted in Table 7 We have dereddened the observed slopes using the extinctions described by Luhman et al. (2009b) and the reddening law from Flaherty et al. (2007), except for members that lack reliable estimates of extinctions. The observed and dereddened values of $\alpha_{2-8}, \alpha_{2-24}, \alpha_{3.6-8}$, and $\alpha_{3.6-24}$ are presented in Table[7. All known members of Taurus are included in Table[7. even those for which none of these slopes could be computed. The classifications for the latter sources are determined from other data, as discussed at the end of this section.

Histograms of the dereddened spectral slopes from Table 7 are shown in Figure [5. The observed slopes are used for stars that lack extinction estimates. Like the data in the color-color diagrams, the slopes form a narrow group of blue sources and a broader distribution of redder objects, corresponding to stellar photospheres and stars with disks, respectively. To better distinguish between these two populations, we plot the slopes as a function of spectral type in Figure 6 . Our adopted spectral types are listed with the spectral slopes in Table 7. (Cowlev 1972; Hartigan et al. 1994; Strom \& Strom 1994; Kenvon \& Hartmann 1995; Torres et al. 1995; Wichmann et al. 1996; Briceño et al. 1998, 2002; Kenyon et al. 1998; Luhman \& Rieke 1998; Malfait et al. 1998; Duchêne et al. 1999; White et al. 1999; Martín 2000; Martín et al. 2001; Steffen et al. 2001; White \& Ghez 2001; Hartigan \& Kenvon 2003; Muzerolle et al.|2003; Walter et al.|2003; White \& Basri 2003; Luhman 2004b, 2006; Luhman et al. 2003a, 2006, 2009a.b; Guieu et al. 2006; Slesnick et al. 2006; Beck 2007; Prato et al. 2009). The sequences of class III stars are narrow and well-separated from the redder population of class $0 / \mathrm{I} / \mathrm{II}$ sources. The class III sequence is tighter at $8 \mu \mathrm{m}$ while the separations from the disk-bearing stars are larger at $24 \mu \mathrm{m}$. The photospheric slopes also vary with spectral type, particularly for $\alpha_{2-8}$. All of these characteristics apply to the data for Chamaeleon I from Luhman et al. (2008a). Indeed, we find that the photospheric sequences for Taurus and Chamaeleon I are very similar. As a result, we adopt the same thresholds for distinguishing between classes II and III that were defined by Luhman et al. (2008a), except that we have used the colors of young field $\mathrm{L}$ dwarfs from Luhman et al. (2009b) to revise the boundaries at L0.

We have used the spectral slopes in Figure 6 to classify the Taurus members that were not already assigned to classes 0 and I in $\S 3.1$ based on previous detections of envelopes. Stars below the thresholds in Figure 6 are classified as class III while redder stars with $\alpha \leq 0$ are designated as class II (Lada 1987). Sources with $\alpha>0$ are labeled as "class I?" if no other data are available to verify the presence of an envelope. Unlike in our study 
of Chamaeleon I, we do not employ the "flat-spectrum" class $(-0.3 \leq \alpha \leq 0.3$, Greene et al. 1994) because most Taurus members within this range of slopes have been observed with mid-IR spectroscopy, which is sensitive to the presence of envelopes and thus discriminates fairly reliably between classes I and II (Furlan et al. 2006, 2008). If a star exhibits excess emission at $24 \mu \mathrm{m}$ but not at $8 \mu \mathrm{m}$, we classify it as class II. A few objects that are slightly bluer than the II/III thresholds at $24 \mu \mathrm{m}$ and are labeled as class III may have small amounts of excess emission. The SEDs of these stars are discussed in $\S \mathbf{7}$.

We discuss in more detail the classifications of several sources, particularly ones for which the four spectral slopes disagree with each other or with classifications derived from previous mid-IR spectroscopy. Although IRAS $04385+2550$, IRAS 04301+2608, IRAS 04260+2642, CZ Tau, CoKu Tau/4, and 2MASS J04210795+2702204 have $\alpha_{3.6-24}>0$, we classify them as class II rather than class I based on the absence of signatures of envelopes (e.g., strong silicate and ice absorption features) in IRS spectra from D'Alessio et al. (2005), Furlan et al. (2006), and unpublished observations in Spitzer programs PID $=2,3303$, and 30765 (D. Watson, private communication). The 8 and $24 \mu \mathrm{m}$ slopes for 2MASS J04381486+2611399 imply class I and class II, respectively. It is probably a class II object since high-resolution images and mid-IR spectroscopy do not show any evidence of an envelope, indicating that it is not a class I source (Luhman et al. 2007a). Those data do reveal an edge-on disk, which is the cause of its red SED. Although the dereddened $24 \mu \mathrm{m}$ slopes of 2MASS J04194657+2712552 are slightly below the I/II threshold of $\alpha=0$, we list it as "class I?" since it has the reddest SED of any known member of Taurus with a measured spectral type later than M6 (excluding the edge-on system 2MASS J04381486+2611399). For IRAS $04187+1927, \alpha_{2-8}$ and $\alpha_{3.6-8}$ produce different classifications (II vs. I). Slopes extended to $24 \mu \mathrm{m}$ are unavailable since this star was not observed by MIPS. We have assigned it to class II based on IRS data (Furlan et al. 2006). J1-4872 A and B are class II based on $\alpha_{2-8}$ but are class III according to the other slopes. We adopt the latter classification for these stars because of the possibility of variability between the dates of the $K_{s}$ and Spitzer observations. For the same reason, we classify CIDA-9 using $\alpha_{3.6-8}$ (class II) rather than with $\alpha_{2-8}$ (class I). Although 2MASS J04373705+2331080 is slightly redder in $\alpha_{2-8}$ than the II/III threshold, this color excess is not reliable given the large uncertainty in the measurement of $K_{s}$. When compared to $3.6 \mu \mathrm{m}$, the 5.8 and $8.0 \mu \mathrm{m}$ measurements for this object do not exhibit significant excess emission relative to stellar photospheres (Luhman et al. 2009b), indicating that it is a class III source. Similarly, V710 Tau B has a small excess at $8 \mu \mathrm{m}$, but the significance of this excess is marginal since the uncertainty in the $8 \mu \mathrm{m}$ measurement is large. We classify this star as "class II?". IRAS $04173+2812$ is class I and class II according to the 8 and $24 \mu \mathrm{m}$ slopes, respectively. We adopt the $24 \mu \mathrm{m}$ classification since the presence of an envelope is more easily detected at longer wavelengths.

Finally, we discuss the classifications of the Taurus members for which none of the four spectral slopes in Table 7 could be computed because the necessary Spitzer photometry is not available. We could not measure photometry at 8 and $24 \mu \mathrm{m}$ for V892 Tau, RY Tau, T Tau N, and $\mathrm{AB}$ Aur because they are either saturated or outside of the images. We assign these stars to class II based on the mid-IR spectroscopy from Furlan et al. (2006). 2MASS J04390637+2334179 was observed at 3.6 and $5.8 \mu \mathrm{m}$ but not at 4.5 and $8.0 \mu \mathrm{m}$. The photometry in the former two bands and a non-detection at $24 \mu \mathrm{m}$ indicate that this star is class III. Six known members of Taurus were not observed by IRAC in any of its bands. Two of these stars, 2MASS J04381630+2326402 and 2MASS J04385871+2323595, were within the field of view of MIPS images, but were not detected. The detection limits at $24 \mu \mathrm{m}$ are sufficiently faint to demonstrate that these stars are class III. One star, MWC 480 , is known to be a class II source from previous observations (Mannings et al. 1997). The remaining three sources have weak $\mathrm{H} \alpha$ emission (Luhman 2004b, 2006; Luhman et al. 2009b; Slesnick et al. 2006), suggesting that they are not undergoing significant accretion. We classify these stars as "class III?".

The SED classifications for all known members of Taurus are presented in Table 7, For multiple systems that are unresolved by IRAC, these classifications apply to the component that dominates at mid-IR wavelengths. However, it is possible for different components to dominate at different IR wavelength ranges, as in the case of T Tau N and S (Furlan et al. 2006). For that system, the designation of class II in Table 7 refers to T Tau N, whereas the southern component may be a class I source (Furlan et al. 2006).

\section{DISK FRACTION}

We would like to use our tabulation of SED classifications in Taurus to measure the fraction of members that have disks as a function of stellar mass. However, some of the known members of Taurus were originally discovered because they exhibited evidence of disks. As a result, the census of Taurus could be biased in favor of stars with disks, in which case the disk fraction computed from all known members would not be representative of the stellar population. To address this possibility, we have measured a disk fraction from a sample of members that is likely to be unbiased in terms of disks. For this sample, we have selected all known members within the fields observed during the XMM-Newton Extended Survey of the Taurus Molecular Cloud (XEST, Güdel et al. 2007). These fields have been searched extensively for new members with a variety of methods and offer relatively well-defined completeness limits (Luhman et al. 2009b). As done in Chamaeleon I (Luhman et al. 2008a), we compute the disk fraction as a function of spectral type, which acts as a proxy for stellar mass. For both the XEST fields and all of Taurus, we list in Table 8 the number of members as a function of SED class and spectral type. For members that lack measured spectral types, most of which are class I sources, we estimated the spectral type bins in which they belong by combining their luminosities (e.g., Furlan et al. 2008) with the values predicted by the evolutionary models for an age of 1 Myr. The two class 0 sources are excluded from Table 8

We now consider the question of how to define the disk fraction in a young population. The ultimate objective 
of studies of disk fractions is the measurement of the average lifetime of disks by comparing disk fractions among clusters with different ages (Haisch, Lada, \& Lada 2001). In these comparisons, the ages that are typically adopted for clusters apply only to the class II and III members since the class I sources usually lack the temperature and luminosity measurements that are needed for isochronal ages. If class I sources are younger than stars in classes II and III (e.g., §5), then it would be inappropriate to include them in disk fractions when estimating disk lifetimes. Therefore, we define the disk fraction as $\mathrm{N}(\mathrm{II}) / \mathrm{N}(\mathrm{II}+\mathrm{III})$ in our analysis of Taurus. In comparison, many previous studies have included both classes I and II in their disk fractions. Because the frequency of class I sources decreases rapidly with cluster age, disk fractions for clusters older than Taurus $(\tau>1 \mathrm{Myr})$ do not depend significantly on the treatment of these objects. However, the adopted definition does have a noticeable effect on the disk fractions in the youngest regions.

The values of N(II)/N(II+III) for Taurus from Table 8 are plotted versus spectral type in Figure 7 . The boundaries of the spectral type bins in Figure 7 have been converted to stellar masses by combining the temperature scale of Luhman et al. (2003b) and the evolutionary models of Baraffe et al. (1998) and Chabrier et al. (2000). As shown in Figure 7 the disk fractions for the known members in the XEST fields and across all of Taurus are similar. If the true disk fraction is the same within and outside of the XEST fields, then the agreement in these disk fractions suggests that the total census of Taurus is not strongly biased for or against stars with disks. These disk fractions in Taurus exhibit a dependence on spectral type and stellar mass, declining steadily from $\sim$ $75 \%$ for solar-mass stars to $\sim 45 \%$ for low-mass stars and brown brown dwarfs. For comparison, we have included in Figure 7 the disk fractions measured from Spitzer observations of Chamaeleon I and IC 348 (Lada et al. 2006; Muench et al. 2007; Luhman et al. 2005, 2008a). The fractions for Taurus and Chamaeleon I vary with spectral type in a similar manner while an opposite dependence on spectral type is present in the data for IC 348. Like Taurus and Chamaeleon I, roughly half of its low-mass members have disks, but the disk fraction is much lower among its solar-mass stars. The $\sigma$ Ori cluster closely resembles IC 348 in this respect (Hernández et al. 2007a; Luhman et al. 2008b). Given that Chamaeleon I, IC 348, and $\sigma$ Ori have similar ages (2-3 Myr, Luhman 2007; Luhman et al. 2008b), these differences in disk fractions are presumably tied to the star-forming conditions of these regions. For instance, Luhman et al. (2008a) suggested that the low disk fraction at higher masses in IC 348 relative to Chamaeleon I might be related to the higher stellar density of IC 348 combined with the segregation of the more massive stars toward the center of the cluster. The high and low disk fractions for solar-mass stars in Taurus and $\sigma$ Ori, respectively, provide further support for this hypothesis since the former is even more sparse than Chamaeleon I while the latter is comparable in density to IC 348. Thus, it appears that the disk lifetimes for stars more massive than $0.5 M_{\odot}$ may be shorter in denser clusters.

Finally, we note that the ratio of the number of class II sources to the number of class I sources in Taurus $(\sim 4.4)$ is similar to the values measured in previous studies of Taurus Kenvon \& Hartmann 1995) and in Spitzer surveys of other nearby regions of ongoing star formation $(\tau<5$ Myr, Evans et al. 2009; Gutermuth et al. 2009). This ratio is typically interpreted as the ratio of the lifetimes of these stages. For instance, if an average lifetime of $3 \mathrm{Myr}$ is adopted for class II sources, then the measurement of $\mathrm{N}(\mathrm{I}) / \mathrm{N}(\mathrm{II})$ in Taurus implies a lifetime of 0.7 Myr for class I objects.

\section{SPATIAL DISTRIBUTIONS OF SED CLASSES}

In addition to measuring the disk fraction as a function of stellar mass in Taurus, we can also use our SED classifications to compare the spatial distributions of the SED classes. To arrive at meaningful results in this comparison, the completeness of the stellar census should not vary significantly with SED class. The images of Taurus from Spitzer have demonstrated that the current census has a high level of completeness for classes I and II across most of the cloud complex (Luhman et al. 2006, 2009b). The completeness for class III stars is not well-defined outside of the denser stellar aggregates and may be lower than that of the class I/II sources in those outer areas, but correcting for such incompleteness would reinforce the primary result regarding the class III sources that we are about to describe, which is that they are more widely distributed than the less evolved stars.

The locations of Taurus members in classes I, II, and III are shown on maps of the cloud complex in Figures 8 and 9. The class I/II sources tend to appear near the dense gas, as found previously in Taurus and other starforming regions (Hartmann 2002; Gutermuth et al. 2009, references therein), while the class III stars are more frequently scattered far from the dark clouds. To perform a quantitative comparison of these spatial distributions, we have computed for each star the distance to the nearest known member from any SED class. The median of these distances is $2.2,3.3$, and 5.5 for classes I, II, and III, respectively, which corresponds to $0.09,0.13$, and $0.22 \mathrm{pc}$ at the distance of Taurus. Histograms of the nearest neighbor distances for classes I+II and III are presented in Figure 10. According to a two-sided Kolmogorov-Smirnov test, the probability that those two samples are drawn from the same parent distribution is $0.3 \%$. The probability that classes I and II are drawn from the same distribution is $10 \%$, which does not represent a significant difference.

Spitzer has been used to measure the spatial distributions of young stars in dozens of other star-forming regions (Gutermuth et al. 2009). Because most of those clusters have not been searched extensively for members prior to the Spitzer imaging, and only the disk-bearing members can be identified with mid-IR photometry, the Spitzer surveys have primarily measured the spatial distributions of classes I and II. For instance, the nearest neighbor distances from Gutermuth et al. (2009) were computed among class I/II sources without the inclusion of class III stars. To enable a comparison of our results to those of Gutermuth et al. (2009), we have computed nearest neighbor distances in Taurus in the same manner. We find that the median of the distance to the nearest member in classes I or II is 2.2, 4.1, and 3'.6 for classes I, II, and I+II, respectively, or $0.09,0.17$, and $0.15 \mathrm{pc}$ at the distance of Taurus. The class II sources again ap- 
pear to be more widely distributed than the protostars. The probability that the nearest neighbor distances for classes I and II are drawn from the same distribution is $2 \%$. Thus, the exclusion of the class III stars has enhanced the difference between the class I and II distributions. A marginally significant difference of this kind also was found by Gutermuth et al. (2009) in their analysis of 36 young clusters.

The median of the nearest neighbor distances for the class I+II sample in Taurus, $0.15 \mathrm{pc}$, is twice as large as the average median for the clusters from Gutermuth et al. (2009), which is consistent with the measurement of larger protostellar envelopes in Taurus than in denser clusters like Ophiuchus (Motte et al. 1998). For several clusters, Gutermuth et al. (2009) found that the distributions of nearest neighbor distances among classes I and II peaked near $0.02-0.05$ pc, while the distribution in Taurus reaches a sharp maximum in the lowest bin below $15^{\prime \prime}$, or $0.01 \mathrm{pc}$, as shown in the top panel of Figure 10. This difference is likely a reflection of the close proximity of Taurus relative to the clusters from Gutermuth et al. (2009, $d=140-1700 \mathrm{pc})$, which allows higher spatial resolution. To give the Taurus sample a resolution that is comparable to that from Gutermuth et al. (2009), we can count neighboring members in Taurus that are within $10^{\prime \prime}$ of each other as single sources. By doing so, the maximum in the Taurus distribution moves out to $0.02-0.03 \mathrm{pc}$ and the median increases by $\sim 20 \%$.

Guieu et al. (2007) measured Spitzer photometry for 23 late-type (M5.5-M9.5) members of Taurus and used these data to check for spatial variations in the disk fraction of brown dwarfs. They found that the disk fraction for low-mass objects in their sample was lower in the southern filaments than in northern ones. In comparison, the more massive members did not appear to exhibit the same trend. Guieu et al. (2007) suggested that the spatial dependence of the disk fraction for low-mass sources could result from an older age for southern filaments combined with shorter disk lifetimes for brown dwarfs. We have investigated this issue with our SED classifications. The northern and southern filaments compared by Guieu et al. (2007) can be separated by a declination of $\delta=25^{\circ} 10^{\prime}$ (J2000). We have computed disk fractions on each side of this boundary for low- and highmass members, arriving at $128 / 175=0.73 \pm 0.03$ for $\leq \mathrm{M} 6$ and $15 / 30=0.50 \pm 0.09$ for $>\mathrm{M} 6$ in the north and $75 / 121=0.62 \pm 0.04$ for $\leq \mathrm{M} 6$ and $5 / 22=0.23_{-0.06}^{+0.11}$ for $>\mathrm{M} 6$ in the south 7 . The ratio of southern to northern disk fractions is $0.85 \pm 0.07$ and $0.46_{-0.14}^{+0.23}$ for $\leq \mathrm{M} 6$ and $>$ M6, respectively. Since these ratios differ by only slightly more than $1 \sigma$, we find that there is no significant evidence to indicate that the disk fraction of brown dwarfs varies with position in a different manner than the disk fraction of stars. The suggestion by Guieu et al. (2007) that disk lifetimes are shorter for brown dwarfs also is not supported by the disk fractions measured in older clusters like Chamaeleon I, IC 348, and $\sigma$ Ori that were described in the previous section.

\section{DISK VARIABILITY}

\footnotetext{
7 The statistical errors in the disk fractions are computed in the manner described by Burgasser et al. (2003).
}

A majority of the members of Taurus have been observed more than once with the cameras on board Spitzer. We can use these multi-epoch measurements to measure the mid-IR variability of disks in Taurus.

We have measured photometry at multiple epochs for 201, 206, 214, and 207 members of Taurus at 3.6, 4.5, 5.8 , and $8.0 \mu \mathrm{m}$, respectively. As done in Chamaeleon I by Luhman et al. (2008a), we have quantified the variability of the data in Taurus by computing the difference between each magnitude and the average magnitude for a given object and wavelength $(\Delta m)$. In Figure 11, we present histograms of $\Delta m$ for class III stars and for sources that are class 0, I, or II. Each measurement of $\Delta m$ is weighted by the inverse of the number of measurements so that all members contribute equally to the distributions. The data in Figure11 demonstrate that stars with disks exhibit significantly greater levels of mid-IR variability than diskless stars, which agrees with variability measurements in Chamaeleon I (Luhman et al. 2008a). Among stars that have been observed at multiple epochs in at least two bands, 92 of 144 class $0 / \mathrm{I} / \mathrm{II}$ sources and 16 of 75 class III stars have $\Delta m>0.05$ in one or more bands. If we require $\Delta m>0.05$ in at least two bands, then these fractions become $64 / 144$ and $2 / 75$ respectively. Thus, the difference in the variability fractions is more pronounced when variability in two bands is required, which is a reflection of the fact that the different IRAC bands usually vary simultaneously for stars with disks but not for class III stars.

It is useful to compare our variability statistics for Taurus to those in Chamaeleon I. We have updated the variability measurements from Luhman et al. (2008a) by combining the IRAC photometry from that study with additional data from Luhman \& Muench (2008). Multiple epochs of photometry are available for $49,52,88$, and 76 members of Chamaeleon I at 3.6, 4.5, 5.8, and $8.0 \mu \mathrm{m}$, respectively. For sources that have been observed more than once in at least two bands, 15 of 57 class $0 / \mathrm{I} / \mathrm{II}$ sources and 2 of 48 class III stars exhibit $\Delta m>0.05$ in two or more bands. Thus, the variability fraction for stars with disks is higher in Taurus than in Chamaeleon I $\left(64 / 144=0.44 \pm 0.04\right.$ vs. $\left.15 / 57=0.26_{-0.05}^{+0.07}\right)$. Since Chamaeleon I is older than Taurus, this comparison indicates that the variability of disks decreases with age.

\section{ADVANCED STAGES OF DISK EVOLUTION}

Taurus offers a unique opportunity for studying the advanced stages of disk evolution. Because Taurus is nearby and does not suffer from crowding or bright nebular emission, mid-IR photometry can be measured more accurately for its stellar population than for most other star-forming regions. Furthermore, since most members of Taurus have been classified spectroscopically, we can reliably estimate the SEDs of their underlying stellar photospheres. The high quality of both the observed and photospheric SEDs makes it possible to detect the small levels of IR excess emission that arise from the most evolved disks, even those surrounding low-mass stars and brown dwarfs. Because the stellar population in Taurus is large and contains significant numbers of both protostars and diskless stars, the intervening stages of disk evolution should be sampled with the best available uniformity and number statistics. For these reasons, Taurus was the site of much of the earliest work on 
the advanced stages of disk evolution (Strom et al. 1989; Skrutskie et al. 1990) and is the one region to which most other young clusters are compared.

In this section, we use our Spitzer data in Taurus to refine the classification of the evolutionary phases of disks and to identify the Taurus members that harbor the most evolved disks. We then apply our classification criteria to other young populations that have been observed with Spitzer so that we can constrain the timescale of disk clearing.

\subsection{Terminology}

A variety of names, definitions, and observational criteria for the stages of disk evolution have been employed in previous studies. We describe our adopted terms in this section and develop observational criteria for them in $\oint 7.2$

We define primordial disks as disks that are optically thick at IR wavelengths and have not experienced inner clearing. Observationally, primordial disks in Taurus form a large, continuous population in terms of mid-IR color excesses, as demonstrated in $\S 7.2$. There are several possible mechanisms that may drive the evolution of primordial disks, including giant planet formation and the growth and settling of dust grains (Najita et al. 2007, references therein).

Pre-transitional disks are primordial disks that have formed gaps in which dust is optically thin or absent. These disks exhibit less excess emission at $\lambda \lesssim 10 \mu \mathrm{m}$ than the average primordial disk but still retain strong disk emission at longer wavelengths. Because they are not distinct from the main population of disks in terms of their mid-IR excess emission ( $\S$ 7.2), we treat pretransitional disks as a subset of primordial disks. Examples of pre-transitional disks in Taurus include UX Tau A and LkCa 15 (Furlan et al. 2006; Espaillat et al. 2007b, 2008a).

The IR excesses from members of Taurus do not decrease monotonically from primordial disks to stellar photospheres. Instead, stars in Taurus are found predominantly in two groups that are well-separated from each other in terms of their IR colors (Skrutskie et al. 1990). The gap between these populations does contain a few stars, including those with transitional disks and evolved disks (Skrutskie et al. 1990; Hernández et al. 2007a). Transitional disks have large inner holes, and thus produce little or no emission at shorter IR wavelengths followed by a sudden onset of strong emission at longer wavelengths. Taurus members that are known to harbor transitional disks include CoKu Tau/4, GM Aur, and DM Tau (Rice et al. 2003; Forrest et al. 2004; Quillen et al. 2004; Calvet et al. 2005; D'Alessio et al. 2005), although the inner hole in the disk of CoKu Tau/4 was probably produced by a stellar companion rather than processes associated with disk evolution and planet formation (Ireland \& Kraus 2008). Meanwhile, evolved disks are full disks without inner holes that are in the process of becoming optically thin. As a result, they exhibit weak emission at all mid-IR wavelengths and their spectral slopes do not show the abrupt increase that

\footnotetext{
8 Alternatively, primordial disks can be defined to include the transitional and evolved stages, i.e., any disks that are less evolved than debris disks.
}

is observed in transitional disks. Other terms used for these disks include anemic, thin, weak, and homologously depleted (Lada et al. 2006; Barrado y Navascués et al. 2007; Dahm \& Hillenbrand 2007; Currie et al. 2009a).

An optically thin disk that contains an inner hole could arise when an evolved disk is inwardly truncated or a transitional disk becomes optically thin. We refer to these disks as evolved transitional disks. They produce little or no excess emission at $\lambda<$ $10 \mu \mathrm{m}$ and weak emission at longer wavelengths. The SEDs of evolved transitional disks closely resemble those of debris disks, which are disks of second-generation dust that is generated by collisions among planetesimals (Kenvon \& Bromlev 2005; Rieke et al. 2005; Hernández et al. 2006; Currie et al. 2008; Carpenter et al. 2009).

\subsection{Classification of Evolutionary Stages}

The stages of disk evolution described in the previous section differ from each other in terms of the strength of mid-IR excess emission as a function of wavelength. We can characterize these differences by examining excesses at multiple wavelengths for all members of Taurus that have been observed by Spitzer. In a transitional disk, the wavelength beyond which excess emission appears depends on the size of the inner hole. Therefore, we consider a range of wavelengths covered by Spitzer, namely $4.5,5.8,8.0$, and $24 \mu \mathrm{m}$. To measure the excess emission in each band, we subtract the Spitzer data from photometry at $K_{s}(2.2 \mu \mathrm{m})$, which is short enough in wavelength that the stellar photosphere usually dominates the total flux while red enough that extinction is low. These colors were corrected for extinction in the manner described for the spectral slopes in $\S 3.3$. We plot $K_{s}-[8.0], K_{s}-[5.8]$, and $K_{s}-[4.5]$ versus $K_{s}-[24]$ for class II and III members of Taurus in Figures 12, 13, and 14, respectively. Since both the average excess emission and the photospheric colors vary with spectral type (Figure 6. Luhman et al. 2008a), we divide the data into four ranges of spectral types $(s p)$, consisting of $s p \leq \mathrm{K} 4.5, \mathrm{~K} 4.5<s p \leq \mathrm{M} 2.5$, $\mathrm{M} 2.5<s p \leq \mathrm{M} 5.75$, and $s p \geq \mathrm{M} 6$. We exclude members that lack measured spectral types and that have photometric uncertainties greater than 0.1 mag. The positions of the known pre-transitional and transitional systems UX Tau A, LkCa 15, GM Aur, DM Tau, and $\mathrm{CoKu} \mathrm{Tau} / 4$ are indicated in the color-color diagrams. Since DM Tau was not observed by MIPS, we have substituted the $25 \mu \mathrm{m}$ measurement from IRAS.

As found in previous studies of Taurus (Skrutskie et al. 1990), the IR colors in Figures 12, 13, and 14 exhibit two distinct populations. The gap between them is larger for bands at longer wavelengths and for earlier spectral types, and is nearly absent in $K_{s}-$ [4.5] for late-type stars. We define the large, continuous population of red sources as primordial disks and objects within the gaps as evolved disks, transitional disks, and evolved transitional or debris disks. The approximate locations of these disks within a gap are indicated in Figure 12. The colors in which a transitional disk can be distinguished from the primordial population depends on the size of its inner hole. For instance, a disk that is primordial according to its $4.5-24 \mu \mathrm{m}$ excesses could have a small hole that produces a deficit of excess emission only at shorter wavelengths. 


\subsection{Models of Settled Disks}

We have performed modeling of accretion disks with large degrees of dust settling to assess whether the sources near the bottom of the primordial populations in Figures 12 14 can be explained in terms of optically thick disks. For these calculations, we have employed models of irradiated accretion disks from D'Alessio et al. (2005, 2006). For comparison to the K5-M2 and M3-M5 populations in Taurus, we consider models for two stars that have spectral types of M0 and M4. We adopt stellar masses of 0.7 and $0.2 M_{\odot}$, effective temperatures of 3850 and $3240 \mathrm{~K}$, and stellar radii of 2.3 and $1.4 R_{\odot}$, respectively. The model disks are composed of amorphous silicates and graphite grains with opacities and dust-togas mass ratios from Draine \& Lee (1984). The grain size distribution follows the form $a^{-3.5}$ where $a$ varies between $a_{\min }$ and $a_{\max }$. The upper disk layers contain grains that are similar in size to those in the interstellar medium (i.e., $a_{\min }=0.005 \mu \mathrm{m}$ and $a_{\max }=0.25 \mu \mathrm{m}$ ). In the midplane, the maximum grain size is $1 \mathrm{~mm}$. We adopt an outer disk radius of $300 \mathrm{AU}$ and the inner disk edge or "wall" is located at the radius where the dust sublimation temperature $(1400 \mathrm{~K})$ is reached. The disks are given accretion rates of $10^{-10} M_{\odot} \mathrm{yr}^{-1}$, a viscosity parameter $(\alpha)$ of 0.01 , and a settling parameter $(\epsilon)$ of 0.001 , where $\epsilon$ is the dust-to-gas mass ratio in the upper disk layers relative to the standard dust-to-gas mass ratio. We selected values for these parameters, particularly $\epsilon$, that are likely to minimize the IR excess emission for comparison to the bluest primordial disks in Taurus.

The IR colors produced by our two models are shown with the data for K5-M2 and M3-M5 members of Taurus in Figures 12 14. For both ranges of spectral types, the model colors appear near the upper boundary of the gaps, indicating that the objects in the main population of disks above the gap can be interpreted as optically thick disks. Thus, the observational criteria defined in the previous section (i.e., gap boundaries) appear to be physically meaningful and consistent with the theoretical distinction between primordial disks and the later stages of disk evolution ( $\S$ 7.1). In addition, our comparison of the observed and model colors demonstrates that Taurus contains a substantial number of optically thick disks that are highly settled, which is not surprising given that $\sim 1 / 3$ of the members of Taurus have fully cleared their inner disks (Table 8).

\subsection{New Transitional and Evolved Disks in Taurus}

The SEDs of Taurus members that have transitional, evolved, and evolved transitional/debris disks according to their 4.5-24 $\mu \mathrm{m}$ colors are presented in Figures 15, 16. and 17. For reference, we include the pre-transitional systems UX Tau A and LkCa 15 in Figure 15. These SEDs consist of our 3.6-24 $\mu \mathrm{m}$ photometry from Spitzer and $J H K_{s}$ data $(1.2-2.2 \mu \mathrm{m})$ from the 2MASS Point Source Catalog. Since DM Tau was not observed at $24 \mu \mathrm{m}$ by MIPS, we have used the $25 \mu \mathrm{m}$ measurement from IRAS in its SED. The sizes of the solid points that comprise the SEDs are equivalent to $\pm 0.12 \mathrm{mag}$. All of the measurements in these SEDs have uncertainties less than this value. To characterize the excess emission in each SED, we compare it to an estimate of the SED of the stellar photosphere, which is based on the average colors of class III stars near the spectral type in question ( $\S \mathrm{A}$ ). We have reddened the photospheric SEDs by combining the extinction estimates described by Luhman et al. $(2009 \mathrm{~b})$ with the reddening laws from Rieke \& Lebofsky (1985) and Flaherty et al. (2007).

Evidence of evolved and transitional disks has been previously presented for several of the stars in Figures [15]17. CoKu Tau/4, GM Aur, and DM Tau were observed with IRS spectroscopy and the resulting data were successfully modeled in terms of disks with inner holes (D'Alessio et al. 2005; Calvet et al. 2005; Furlan et al. 2006). Similar analysis was applied to UX Tau A and LkCa 15, which show evidence of disk gaps (Furlan et al. 2006; Espaillat et al. 2007b). Furlan et al. (2006, 2009) detected excess emission longward of $12 \mu \mathrm{m}$ in an IRS spectrum of V819 Tau. Furlan et al. (2006) also reported a tentative detection of excess emission beyond $20 \mu \mathrm{m}$ in an IRS spectrum of V410 X-ray 3, but that excess is no longer present in a new reduction of those observations (Furlan et al. 2009). Nevertheless, we do find that V410 X-ray 3 exhibits a small excess of $\sim 0.4 \mathrm{mag}$ at $24 \mu \mathrm{m}$, which is below the detection limit of the IRS data (Furlan et al. 2009). Andrews \& Williams (2005) suggested that FW Tau could be a transitional disk based on a detection at submillimeter wavelengths. The spatial resolution of those observations is insufficient to determine whether the source of the submillimeter emission is the 0.08 binary FW Tau $\mathrm{A}+\mathrm{B}$ or their 2 .' 3 companion FW Tau $\mathrm{C}$, which is known to have a disk (White \& Ghez 2001). Although MIPS cannot fully resolve a pair with this separation, the $24 \mu \mathrm{m}$ emission does appear to be centered on the former rather than the latter, indicating that FW Tau A+B indeed may be responsible for the submillimeter emission. If so, the disk around $\mathrm{FW}$ Tau $\mathrm{A}+\mathrm{B}$ is an evolved transitional disk or debris disk rather than a transitional disk based on the small size of the $24 \mu \mathrm{m}$ excess. The SED of IRAS 04125+2902 in Figure 15] was also shown in the study that confirmed its membership in Taurus (Luhman et al. 2009b). The remaining stars in Figures 15] 17 have not been previously identified as having disks in advanced stages of evolution. We note that one star in Figure 17 (XEST 17-036) and the stars in Figure 17 have $24 \mu \mathrm{m}$ excesses that are too small to be classified as class II with the II/III thresholds that were adopted in Figure 6.

Additional data are needed to definitively assess the nature of our new candidates for transitional and evolved disks. For instance, the SEDs in Figure 17 could arise from both evolved transitional disks and debris disks. When SEDs of this kind are observed for early-type stars, they are cited as convincing evidence of debris disks since the dust in an (optically thin) evolved transitional disk would be removed very quickly. However, a source of dust replenishment may not be necessary to explain the SEDs in Figure 17 since the dust removal timescale is roughly comparable to the age of Taurus for low-mass stars (Backman \& Paresce 1993; Currie et al. 2009b). Because debris disks are depleted of gas, measurements that trace gas can help distinguish between debris disks and evolved transitional disks. The strengths of the $\mathrm{H} \alpha$ emission lines from the candidate evolved transitional/debris systems do not indicate the presence of active accretion, and thus allow 
for the possibility of gas depletion (Strom et al. 1989; Kenvon et al. 1998; White \& Ghez 2001; Muzerolle et al. 2003; Luhman 2004b; Luhman et al. 2009b).

Binarity also is relevant to the interpretation of our candidates. Excess emission that appears only at longer wavelengths can arise from the disk of an unresolved lowmass companion rather than from a disk with an inner hole. Even if the latter is present, the inner truncation of the disk may be caused by interactions with a stellar companion rather than processes associated with disk evolution (Guenther et al. 2007; Ireland \& Kraus 2008). Multiplicity measurements are required to address these possibilities. Finally, millimeter and submillimeter observations would be valuable for verifying the presence of disks for the stars with the smallest $24 \mu \mathrm{m}$ excesses (Andrews \& Williams 2005) and for resolving inner holes (Brown et al. 2008; Dutrey et al. 2008; Hughes et al. 2007, 2009) while mid-IR spectroscopy would provide the high-resolution SEDs that are necessary for detailed modeling of the disks (Calvet et al. 2005; D'Alessio et al. 2005; Espaillat et al. 2007b).

As with V819 Tau and V410 X-ray 3, Furlan et al. (2006) found excess emission at long wavelengths in their IRS spectrum of HBC 427. However, they suggested that a star at a distance of $15^{\prime \prime}$ from HBC 427 may have contributed light into the IRS slit during the observations of $\mathrm{HBC} 427$, resulting in a spurious excess. To assess this possibility, we have inspected the MIPS $24 \mu \mathrm{m}$ images of HBC 427 . We find that the nearby star cited by Furlan et al. (2006) is sufficiently faint and well-resolved that it probably did not contaminate the IRS spectrum of HBC 427. However, another object that is $9^{\prime \prime}$ from HBC 427, corresponding to HBC $427 / 1$ from Massarotti et al. (2005), is $\sim 1.3 \mathrm{mag}$ brighter than $\mathrm{HBC} 427$ at $24 \mu \mathrm{m}$ and very likely is the source of the apparent excess emission in the IRS data. This conclusion is supported by the fact that our $24 \mu \mathrm{m}$ photometry of HBC 427 does not exhibit excess emission. The contaminating source is probably a galaxy based on its near- and mid-IR colors. Furlan et al. (2009) arrived at the same conclusion through analysis of the MIPS data as well as peak-up images from IRS.

\subsection{Comparison to Clusters at 2-10 Myr}

Imaging with the Spitzer Space Telescope has been widely used to classify the evolutionary stages of disks in nearby clusters and associations. We can apply the observational criteria developed for Taurus to these populations to provide the uniformly-derived classifications that are necessary for meaningful comparisons among them. We have selected clusters and associations that were observed with both IRAC and MIPS down to spectral types of $\sim \mathrm{M} 2\left(M \sim 0.5 M_{\odot}\right)$ and that are older than Taurus but within the era of primordial disks $(\tau \sim 2$ $10 \mathrm{Myr})$. For each region, we will plot $K_{s}-$ [5.8] and $K_{s}-$ [8.0] versus $K_{s}-$ [24] for spectral types of K5-M2 and M3-M5. The Spitzer data for a few clusters do not reach the latter range of types. We begin by showing the colors for the class II and class III sources in Taurus in the same format in Figure 18. We have defined boundaries in Figure 18 that follow the upper edges of the color gaps in Taurus, which will be used for identifying transitional and evolved disks in the other clusters. We find that the same boundaries are adequate for the two ranges of spectral types. These boundaries are defined as lines connecting $\left(K_{s}-[5.8], K_{s}-[8.0]\right.$, $\left.K_{s}-[24]\right)=(1,1.55,3.4),(0.7,1.15,4.1)$, and $(0.7,1.15,6)$.

\subsubsection{Chamaeleon I}

The dereddened values of $K_{s}-[5.8], K_{s}-$ [8.0], and $K_{s}-[24]$ for K5-M2 and M3-M5 members of Chamaeleon I ( $\tau \sim 2-3$ Myr, Luhman 2007) are plotted in Figure 19. The Spitzer data are from Luhman et al. (2008a) and Luhman \& Muench (2008) and the extinction estimates that were used for dereddening the colors are from Luhman (2007) and Luhman \& Muench (2008). We adopt the $K_{s}$ data from 2MASS and Luhman (2007). As shown in Figure 19, the distribution of colors in Chamaeleon I closely resembles that in Taurus. The same gap in colors is present in both regions. Several stars below the boundaries in Figure 19 have been previously recognized as possible transitional systems, consisting of CS Cha, T35, C7-1, CHXR 22E, CHXR 71, and CHXR 76 (Takami et al. 2003; Damianov et al. 2007; Espaillat et al. 2007a; Luhman et al. 2008a; Kim et al. 2009). Using our terminology ( $\S$ 7.1, Figure 12) and the colors in Figure 19, we classify CS Cha, T35, and CHXR 22E as transitional disks, C7-1 and CHXR 71 as evolved disks, and CHXR 76 as an evolved transitional disk or a debris disk. In addition, we identify CHSM 9484 as a new candidate for an evolved disk. The ratio of the number of transitional and evolved disks to the number of primordial disks in Figure 19 is $7 / 56$, which is similar to the value of 15/98 in Taurus for K5-M5. SZ Cha, T21, T25, T54, and T56 also exhibit evidence of transitional disks (Kim et al. 2009), but are absent from Figure 19 because measurements with IRAC or MIPS are unavailable, or they have spectral types earlier than K5.

\subsection{2. $I C 348$}

The dereddened colors for K5-M2 and M3-M5 members of IC $348(\tau \sim 2-3$ Myr, Luhman et al. 2003b) are shown in Figure 20. The Spitzer measurements and extinction estimates are from Lada et al. (2006), Muench et al. (2007), and Currie et al. (2009b) and the $K_{s}$ data are from 2MASS. The sensitivity of the $24 \mu \mathrm{m}$ images of IC 348 is lower than that in Taurus and Chamaeleon I because of brighter background emission. As a result, those images do not detect most of the stellar photospheres for spectral types later than K5, making it more difficult to characterize the distribution of colors. However, the available data that are shown in Figure 20] are consistent with the presence of a gap in colors that is similar to the ones observed in Taurus and Chamaeleon I. According to Figure 20, sources 67, 72, 133, and 190 from Luhman et al. (2003b) have transitional disks and source 176 from that study has an evolved disk. Source 72 was previously noted as a possible transitional disk by Lada et al. (2006). Several additional stars appear below the boundaries in Figure 20. but their candidacy as evolved disks or transitional disks is questionable since the uncertainties in their $24 \mu \mathrm{m}$ data are large (0.1-0.5 mag, Currie et al. 2009b). Currie et al. $(2009 \mathrm{~b})$ suggested that the frequency of transitional and evolved disks is higher in IC 348 than in Taurus. However, we find that the frequencies are consistent with each other when we apply the same observational criteria to both regions. For instance, even if the IC 348 members 
with uncertain $24 \mu \mathrm{m}$ photometry are included, the ratio of the number of transitional and evolved disks to the number of primordial disks is 15/98 in Taurus and $20 / 83$ in IC 348 for spectral types of K5-M5 based on the thresholds adopted in Figure 20.

$$
\text { 7.5.3. } \sigma \text { Ori }
$$

For the $\sigma$ Ori cluster $(\tau \sim 3 \mathrm{Myr}$, Hernández et al. 2007a, references therein), we have adopted the membership list that was compiled by Luhman et al. (2008b). The colors measured in that study and by 2MASS for K5-M2 and M3-M5 stars are plotted in Figure 21 We have not corrected the colors for extinction since the cluster exhibits little reddening. Since many members of $\sigma$ Ori have not been spectroscopically classified, we assume that stars with $2.1<V-J<3.5$ and $3.5 \leq$ $V-J<5.7$ have types of K5-M2 and M3-M5, respectively. For stars that lack $V$-band measurements, we apply criteria of $J-K_{s}>0.7$ and $J<11.7$ for K5-M2 and $J-K_{s}>0.7$ and $11.7 \leq J<14.5$ for M3-M5. We adopt measurements of $V$ and $J$ from Hernández et al. (2007a) and 2MASS, respectively.

The distribution of colors in Figure 21 for $\sigma$ Ori is similar to that of Taurus, showing a prominent gap between stellar photospheres and the main population of disks. Our classifications of transitional and evolved disks differ from those of Hernández et al. (2007a) in only a few cases. Hernández et al. (2007a) classified sources 540 and 1156 from their study as class II while we identify them as possible transitional disks based on Figure 21. Inspection of the SEDs of these stars from Hernández et al. (2007a) tends to confirm our designations. Sources 615, 818, and 1267 appear above our adopted thresholds in Figure 21 but were listed as evolved or transitional systems by Hernández et al. (2007a). The latter two stars exhibit color excesses in all of the IRAC bands relative to the $K_{s}$ but not in [3.6] - [4.5] and [4.5] - [5.8], which can be explained by variability between the $2 \mathrm{MASS}$ and Spitzer observations. Thus, these two stars probably do have transitional disks as found by Hernández et al. (2007a).

\subsection{4. $\operatorname{Tr} 37$}

For Trumpler $37(\operatorname{Tr} 37, \tau \sim 4$ Myr, Sicilia-Aguilar et al. 2005), we have adopted the membership list, extinction estimates, and spectral types that were presented by Sicilia-Aguilar et al. (2005), the Spitzer photometry measured by Sicilia-Aguilar et al. (2006), and the $K_{s}$ data from 2MASS. The dereddened colors for K5-M2 members of $\operatorname{Tr} 37$ are shown in Figure 22. We have omitted stars for which extinctions were not estimated by Sicilia-Aguilar et al. (2005).

The data in Figure 22 are incomplete at bluer colors because of the detection limit of the $24 \mu \mathrm{m}$ image, which does not reach stellar photospheres between K5 and M2. Nevertheless, the available colors are consistent with a gap between primordial disks and photospheres that is similar to the one in Taurus. Sicilia-Aguilar et al. (2006) suggested that the median SED for stars with disks in Tr 37 exhibits less excess emission than the median SED in Taurus. However, we find that the median SEDs do not differ significantly when we utilize our photometry in Taurus. For instance, the dereddened colors for class II K5-M2 stars have median values of $K_{s}-[5.8]=1.38$ and
$1.50, K_{s}-[8.0]=2.20$ and 2.06 , and $K_{s}-[24]=5.16$ and 5.11 for Taurus and $\operatorname{Tr} 37$, respectively.

Sicilia-Aguilar et al. (2006) defined transitional objects as stars that have photospheric colors at $\lambda \leq 4.5 \mu \mathrm{m}$ and excess emission at longer wavelengths. They identified as many as 14 sources of this kind in $\operatorname{Tr} 37$. Eleven of these stars were not detected at $24 \mu \mathrm{m}$ and one additional star is outside of the range of spectral types that we are considering. As a result, these 12 sources are absent from Figure 22. Among the remaining two candidate transitional disks from Sicilia-Aguilar et al. (2006), we classify one as a primordial disk (14-11) and the other as a transitional disk (13-52) based on our adopted criteria.

\subsection{5. $N G C$ 2362}

For NGC $2362(\tau \sim 5$ Myr, Balona \& Lanev 1996; Moitinho et al. 2001; Dahm 2005; Mayne \& Naylor 2008), we have used the spectral types measured by Dahm (2005) and estimated from colors by Currie et al. (2009a), the IRAC and MIPS photometry from Currie et al. (2009a), and the $K_{s}$ photometry from 2MASS. The IRAC data from Currie et al. (2009a) are similar to those measured from the same images by Dahm \& Hillenbrand (2007). Before using these data for our color-color diagrams, we inspected the $24 \mu \mathrm{m}$ images from MIPS for the faintest detections that were presented by Currie et al. (2009a). In contrast to Currie et al. (2009a), we find that source 1091 from Irwin et al. (2008) was not detected in the MIPS images. The nearest intensity peak in the MIPS data is $\sim 3 . \prime 5$ from the optical and IRAC coordinates of 1091, which is too far to correspond to a $24 \mu \mathrm{m}$ counterpart. We also find that source 663 from Irwin et al. (2008) was not detected by MIPS. Sources 809 and 931 from Irwin et al. (2008) and sources 41 and 63 from Dahm \& Hillenbrand (2007) were detected with signal-to-noise ratios of $\lesssim 2-3$, which is too low for useful photometry. We exclude from our analysis the $24 \mu \mathrm{m}$ measurements for these six stars that were reported by Currie et al. (2009a). After doing so, there remain 29 stars that were found to have disks by Dahm \& Hillenbrand (2007) and Currie et al. (2009a) and that have $24 \mu \mathrm{m}$ photometry. The colors of the 25 stars between K5 and M2 are plotted in Figure 22. We have not corrected the colors for reddening since little extinction is present in NGC $2362\left(A_{V}<1\right.$, Moitinho et al. 2001). The four disk-bearing stars outside of the range K5-M2 consist of one K3 star and three M3 stars. We classify the former as a transitional disk and the latter stars as primordial disks with our criteria developed for Taurus. We include the classifications of these four stars with those of the K5-M2 stars in the statistics that we are about to describe.

Sixteen of the 47 disk-bearing stars that were identified by Dahm \& Hillenbrand (2007) have $24 \mu \mathrm{m}$ photometry. These MIPS-detected sources consist of 11 primordial disks and five weak (evolved) disks according to the criteria adopted by Dahm \& Hillenbrand (2007). Currie et al. (2009a) classified only four of the 16 disks as primordial, suggesting that Dahm \& Hillenbrand (2007) overestimated the number of primordial disks. In comparison, we classify 15 of these disks as primordial since they appear above the boundaries indicated in Figure 22 and thus fall within the region inhabited by the main 
population of disks in Taurus.

Currie et al. (2009a) presented a sample of 35 stars with MIPS photometry and detections of disks, which they classified as six primordial disks, 17 homologouslydepleted (evolved) disks, and 12 transitional disks. Among the latter two categories, we find that six stars have unreliable MIPS photometry (see above) and nine stars appear above both of our thresholds for primordial disks in Figure 22. Currie et al. (2009a) underestimated the number of primordial disks because they defined these disks to be close to the median SED of disks in Taurus without accounting for the spread in the colors of the Taurus disks.

As seen in a comparison of Figures 18 and 22 , the primordial disk population in NGC 2362 is weighted more heavily toward bluer colors, or flatter disks, than the primordial disks in Taurus. This feature suggests the youngest members of NGC 2362 are old enough that most of them have already evolved from flared disks to settled disks. In other words, if the reddest (and youngest) primordial disks in Taurus were removed from Figure 18, the resulting distribution of colors would resemble the distribution in NGC 2362. The similarity in those distributions of colors extends to the evolved and transitional regime. For instance, the ratio of the number of evolved and transitional disks to the number of flat primordial disks $\left(K_{s}-[8.0]<2, K_{s}-[5.8]<1.4\right)$ in NGC $2362(7 / 16)$ does not differ significantly from the value in Taurus $(7 / 22)$ for the range of spectral types in the NGC 2362 sample of disks (K4.5-M3).

Currie et al. (2009a) concluded that the ratio of evolved and transitional disks to all primordial disks is much higher in NGC 2362 than in Taurus, which they interpreted as evidence for a long timescale for the evolved/transitional phases $(\S(7.6)$. However, that ratio is only meaningful if the rate of star formation has been roughly constant from the earliest point at which stars enter the class II stage (reddest and most flared disks) through the evolved and transitional stages. In other words, without ongoing star formation in a cluster, the ratio of evolved/transitional disks to primordial disks will naturally increase over time as the supply of primordial disks is exhausted. It is clear that star formation is not continuing at a constant rate in NGC 2362 based on the paucity of red primordial disks in Figure 22 and the positions of cluster members on the color-magnitude diagram from Moitinho et al. (2001). The appropriate metric for comparing two clusters with different star formation histories is the number ratio of evolved/transitional disks to stars in the stage immediately preceding stage, i.e., primordial disks that have experienced large degrees of dust settling, which is the ratio that we employed in the previous paragraph. In addition, Currie et al. (2009a) counted as evolved/transitional disks the six stars that we excluded because of questionable MIPS detections, and they did not apply the same observational criteria for evolved, transitional, and primordial disks to both NGC 2362 and Taurus, making their comparison of the two populations less reliable.

\subsection{6. $\gamma$ Velorum, 25 Ori, and Orion OB1b}

For the $\gamma$ Velorum, 25 Ori, and Orion OB1b stellar populations $(\tau \sim 5,7-10$, and $5 \mathrm{Myr}$, Briceño et al. 2007; Hernández et al. 2008), we have adopted the Spitzer pho- tometry from Hernández et al. (2007b, 2008) and the $K_{s}$ data from 2MASS. We have used the spectral types measured by Briceño et al. (2005, 2007) and Downes et al. (2008) when possible, and otherwise estimated them from $V-J$ using $V$ data from Hernández et al. (2007b, 2008) and $J$ photometry from 2MASS. The colors for K5-M2 and M3-M5 members of the three populations are presented in Figures 23 and 24. We have not dereddened the colors since these stars exhibit little extinction $\left(A_{V}<1\right.$, Pozzo et al. 2000; Briceño et al. 2005; Hernández et al. 2006).

Like NGC 2362, the primordial disks in $\gamma$ Velorum are dominated by bluer, flatter disks. The number ratio of transitional and evolved disks to those flatter disks $(4 / 7)$ is consistent with the one described in $\S 7.5 .5$ for Taurus. The classifications based on our adopted criteria agree with those of Hernández et al. (2008) with the exception of sources 115 and 414 from that study. These stars appear on or above the lower boundaries for primordial disks in Figure 23 while Hernández et al. (2008) classified them as evolved disks.

The samples of disks in 25 Ori and OB1b are too small for quantitative comparisons to Taurus. In both samples, a majority of the disks are primordial. We classify source 905 from Hernández et al. (2007b) as a primordial disk since it is slightly above the boundaries in Figure 23 while Hernández et al. (2007b) classified it as an evolved disk. Similarly, source 585 was listed as a transitional disk by Hernández et al. (2007b), but its $5.8 \mu \mathrm{m}$ excess is slightly too large to satisfy our adopted criteria for this category. Our remaining classifications in 25 Ori and OB1b agree with those of Hernández et al. $(2007 \mathrm{~b})$. One of the transitional disks in 25 Ori, source 1200 from Hernández et al. (2007b), has been studied in detail through IRS and $\mathrm{H} \alpha$ spectroscopy and a comparison of its SED to the predictions of disk models (Espaillat et al. 2008b).

$$
\text { 7.5.7. } \eta \text { Cha }
$$

For the $\eta$ Cha association $(\tau \sim 6 \mathrm{Myr}$, Mamaiek et al. 1999; Lawson et al. 2001; Luhman \& Steeghs 2004), we have adopted the spectral types from Luhman \& Steeghs (2004), the IRAC photometry from Megeath et al. (2005), and the $K_{s}$ photometry from 2MASS. Gautier et al. (2008) and Sicilia-Aguilar et al. (2009) presented $24 \mu \mathrm{m}$ photometry based on the same MIPS images of $\eta$ Cha. Sicilia-Aguilar et al. (2009) noted a systematic difference between the data from the two studies. After reducing those images with the methods that were employed for Taurus, we arrive at photometry that is an average of $0.1 \mathrm{mag}$ brighter and $0.05 \mathrm{mag}$ fainter than the measurements from Gautier et al. (2008) and Sicilia-Aguilar et al. (2009), respectively. In addition, we have measured photometry from all other MIPS images of $\eta$ Cha, which were obtained through Spitzer programs $\mathrm{PID}=40,84,173$, and 50316. We present our $24 \mu \mathrm{m}$ measurements in $\S \mathrm{A}$ and adopt them for our analysis of $\eta$ Cha, using the weighed average for each star that was observed more than once. The colors for K5-M2 and M3-M5 members of the association are shown in Figure 24. No correction for reddening has been applied to these data $\left(A_{V}<1\right.$, Luhman \& Steeghs 2004). Because of their close proximity to the Sun $(d \sim 100 \mathrm{pc}$, Mamajek et al. 1999), all of the members of $\eta$ Cha that 
were within the $24 \mu \mathrm{m}$ images were detected in those data. As a result, the distributions of colors in Figure 24 do not suffer from incompleteness among the stellar photospheres, although they are poorly sampled because of the small size of the association.

Through analysis of their IRAC data, Megeath et al. (2005) found that six members of $\eta$ Cha have excess emission at $8 \mu \mathrm{m}$. Four of these stars (M4-M5.75) exhibit little or no excess at $\lambda<6 \mu \mathrm{m}$, which Megeath et al. (2005) interpreted as evidence of inner holes. The resulting number ratio of transitional disks to all disks with $8 \mu \mathrm{m}$ excesses (4/6) appeared to differ significantly from that in Taurus. However, through our full census of disks in Taurus, we find that three of the four stars cited as transitional disks in $\eta$ Cha have colors that fall within the main population of disks in Taurus, as demonstrated by a comparison of Figures 18 and 24, and that can be explained in terms of optically thick disks with large degrees of dust settling ( $\S$ 7.3, Ercolano et al. 2009). Only one of these stars, RECX-5, qualifies as a transitional disk based on our adopted criteria.

Sicilia-Aguilar et al. (2009) studied the disk population in $\eta$ Cha by combining the IRAC data with MIPS photometry and IRS spectroscopy. They identified four stars (M1.75-M4.5) that have photospheric colors at $\lambda<6 \mu \mathrm{m}$ and excess emission at longer wavelengths. Two of these disks were classified as transitional by Megeath et al. (2005) while the remaining two stars lack excesses in any of the IRAC bands. For low-mass stars, Sicilia-Aguilar et al. (2009) suggested that the absence of excess emission at $\lambda<6 \mu \mathrm{m}$ indicates that the disk has an inner hole, or perhaps is relatively flat (Ercolano et al. 2009). In either case, Sicilia-Aguilar et al. (2009) advocated for the classification of these disks as transitional. The resulting number ratio of transitional disks to all disks with $24 \mu \mathrm{m}$ excesses (4/8) appeared to be much larger than the value in Taurus. As a result, Sicilia-Aguilar et al. (2009) concluded that the transitional phase is not rapid relative to the lifetimes of primordial disks.

As discussed in $\S$ 7.1, transitional objects were originally defined as stars whose colors appear within a gap between stellar photospheres and most stars with disks in Taurus. These sources were interpreted as disks in which the dust in the inner regions is optically thin or completely cleared. The criterion for transitional disks proposed by Sicilia-Aguilar et al. (2009) for low-mass stars - an absence of excess emission at $\lambda<6 \mu \mathrm{m}$ - does not satisfy either of these observational and theoretical definitions since it encompasses stars that are within the main population of disks in Taurus in terms of their IR colors, and since those colors can be explained with optically disks, as shown in $\S \mathbf{7 . 3}$ and Figures 12 14. Furthermore, because the definition of transitional disks from Sicilia-Aguilar et al. (2009) included flat optically thick disks, it does not provide a measurement of the timescale of inner disk clearing.

When we apply our classification criteria to the data for $\eta$ Cha in Figure 24, we find that the association contains five primordial disks, one transitional disk, and two stars that have weak excess emission at $24 \mu \mathrm{m}$, indicating the presence of either evolved transitional disks or debris disks. As done for NGC 2362 ( $\$$ 7.5.5), we compare $\eta$ Cha to Taurus in terms of their number ratios of evolved and transitional disks to the bluest and flattest of the primordial disks. For spectral types of K5 to M5, this ratio is (1-3)/ 4 for $\eta$ Cha and (11-15)/43 for Taurus, where the range of values for each numerator corresponds to the uncertainty in the nature of the disks with weak $24 \mu \mathrm{m}$ emission. These ratios are consistent with each other within the statistical uncertainties.

\subsubsection{Upper Sco}

For the Upper Sco association $(\tau \sim 5 \mathrm{Myr}$, Preibisch \& Mamajek 2008), we have adopted the Spitzer photometry from Carpenter et al. (2006, 2009), the $K_{s}$ data from 2MASS, and the compilation of spectral types and extinctions from Carpenter et al. (2009). The Spitzer observations were performed at 4.5, 8.0, and $24 \mu \mathrm{m}$ and did not include 3.6 and $4.5 \mu \mathrm{m}$. The dereddened values of $K_{s}-[8.0]$ and $K_{s}-[24]$ for K5-M2 and M3-M5 members of Upper Sco are plotted in Figure 25. We have omitted sources that have questionable $24 \mu \mathrm{m}$ data according to Carpenter et al. (2009).

Because of the close proximity of Upper Sco $(d \sim$ 145 pc, Preibisch \& Mamajek 2008), the $24 \mu \mathrm{m}$ images were able to detect the stellar photospheres of a large number of low-mass stars. Like Taurus and the other young populations that we have examined, Upper Sco exhibits a distinct gap between stellar photospheres and redder sources. Our classification criteria suggest that the following seven stars between K5 and M5 have disks that are evolved or transitional: [PBB2002] USco J155729.9-225843, [PBB2002] USco J160525.5-203539, [PBB2002] USco J160600.6-195711, [PBB2002] USco J160622.8-201124, [PBB2002] USco J160643.8-190805, [PBB2002] USco J160827.5-194904, and ScoPMS 31. Carpenter et al. (2009) identified 10 additional K5-M5 stars with smaller $24 \mu \mathrm{m}$ excesses that are candidates for debris disks (see Figure 25). Given that the excesses in these latter sources could also arise from evolved transitional disks, the ratio of the number of evolved and transitional disks to the number of relatively flat primordial disks $\left(K_{s}-[8.0]<2, \S[7.5 .5)\right.$ is $7-17 / 12$. This ratio is higher than the value of $11-15 / 43$ for Taurus, although they are consistent within the statistical errors. A higher ratio in Upper Sco could be explained by a star formation history that is not uniform across the phases represented in the numerator and denominator $\left(\oint \frac{1.5 .5}{17}\right)$. In other words, if the youngest members of Upper Sco are old enough to have begun clearing their disks, then one would expect a ratio of transitional/evolved disks to primordial disks that is larger than that in Taurus.

\subsection{Timescale of Disk Clearing}

The frequency of evolved and transitional disks provides a constraint on the timescale of the clearing of optically thick disks. Based on the small number of transitional disks in Taurus, Skrutskie et al. (1990) concluded that this process occurs rapidly. By combining a value of $3 / 30$ for the number ratio of transitional disks to primordial disks with an average lifetime of $\sim 3$ Myr for primordial disks, they estimated a timescale of $\sim 0.3$ Myr for the transitional phase. Other studies of Taurus have arrived at even shorter timescales $(\tau \lesssim 0.1 \mathrm{Myr}$, Simon \& Prato 1995; Wolk \& Walter 1996). Using our classifications of the Spitzer data in Taurus, we find that the number ratio of evolved and transitional disks to primordial disks 
is 15/98 for spectral types of K5-M5. Thus, we derive a somewhat longer timescale of $\sim 0.45 \mathrm{Myr}$, assuming a primordial disk lifetime of $3 \mathrm{Myr}$. We have overestimated this timescale if some of the stars with weak $24 \mu \mathrm{m}$ emission have debris disks rather than evolved transitional disks, or if stellar companions are responsible for the inner holes in some of the transitional disks (CoKu Tau/4, Ireland \& Kraus 2008).

The timescale of disk clearing has been investigated further through the Spitzer observations of older clusters that were described in $\S 7.5$. Some of those studies have concluded that the frequency of evolved and transitional disks is much higher in older clusters than in Taurus, implying a timescale for this phase that is longer than previous estimates (Currie et al. 2009a; Sicilia-Aguilar et al. 2009). However, in our analysis of the two clusters in question, NGC 2362 and $\eta$ Cha $(\S$ 7.5.5, $\S$ 7.5.7), we found that Currie et al. (2009a) and Sicilia-Aguilar et al. (2009) overestimated the number of evolved and transitional disks because they adopted criteria that encompassed optically thick disks that are relatively flat. In addition, when computing the frequencies of evolved and transitional disks in NGC 2362 and $\eta$ Cha for comparison to Taurus, Currie et al. (2009a) and Sicilia-Aguilar et al. (2009) divided by the number of all primordial disks, whereas only the flatter primordial disks should have been counted given that star formation is no longer occurring in these clusters. In $\oint$ 7.5.5 and $\S$ 7.5.7 we addressed these issues by applying classification criteria that exclude flat optically thick disks from the transitional stage, and by comparing the populations in terms of the ratio of evolved and transitional disks to flatter primordial disks. By doing so, we found that the frequencies of evolved and transitional disks in NGC 2362 and $\eta$ Cha do not differ significantly from the value in Taurus. The other clusters that were examined in $\S 7.5$ also exhibit distributions of color excesses that are consistent with the same timescale of disk clearing that is implied by the data in Taurus.

Based on the large frequency of evolved and transitional disks that they measured in NGC 2362, Currie et al. (2009a) concluded that the timescale of these phases may be comparable to the lifetime of primordial disks. Currie et al. (2009a) attempted to explain how the low number of evolved and transitional disks in Taurus (i.e., the gap in IR colors) is consistent with such a long lifetime for those disks. They suggested that Taurus contains few evolved and transitional disks because most disks in Taurus are too young to have evolved beyond the primordial stage. However, that hypothesis is inconsistent with the fact that a large fraction of the stellar population in Taurus (40\%) has already fully cleared their disks and become class III stars (Table 8). If Taurus is old enough to have a significant number of diskless stars, then the evolved and transitional phases should be heavily populated as well if they have a long timescale.

To account for the existence of class III stars in Taurus, Currie et al. (2009a) contended that the bulk of these stars may have lost their disks through interactions with stellar companions rather than mechanisms associated with the dispersal of disks during the evolved and transitional phases. However, if disruption by companions has been the dominant mechanism for disk removal in Taurus, then the disk fraction as a function of stellar mass should be anti-correlated with binary frequency, but this is not observed in Taurus (Figure 7. Kraus, White, \& Hillenbrand 2006; Luhman et al. $2007 \mathrm{~b})$. In addition, according to the scenario proposed by Currie et al. (2009a), class III stars should have the same ages as class II sources in Taurus. However, class III stars are more widely distributed than members with disks $(\S 5)$, which indicates that they are older on average. In other words, if the fundamental difference between class II and III sources is the absence or presence of tight binaries, then they should share the same spatial distribution. Overall, the suggestion that the lifetime of evolved and transitional disks is comparable to that of primordial disks is incompatible with the observed properties of stars and disks in Taurus. The same conclusion applies to other clusters that also exhibit clear evidence of a paucity of evolved and transitional disks (e.g., Chamaeleon I).

\section{CONCLUSIONS}

We have performed a census of the circumstellar disk population of the Taurus star-forming region $(\tau \sim 1 \mathrm{Myr})$ using mid-IR images obtained with the Spitzer Space Telescope. The results of this study are summarized as follows:

1. We have analyzed nearly all images of the Taurus cloud complex at 3.6, 4.5, 5.8, 8.0, and $24 \mu \mathrm{m}$ that were collected by Spitzer during its cryogen mission. The IRAC and MIPS cameras on board Spitzer each covered a total area $46 \mathrm{deg}^{2}$ and encompassed 346 and 299 members of Taurus, respectively, corresponding to $99 \%$ of the known stellar population. We have presented photometry for all members that were detected in these images.

2. We have used our mid-IR photometry in conjunction with other available observations to determine the likely evolutionary stages of all members of Taurus (classes 0-III). Stars that exhibit evidence of circumstellar envelopes in previous measurements (e.g., IRS spectra) are assigned to classes 0 and I. We have classified the remaining members of Taurus using their mid-IR SEDs, or $\mathrm{H} \alpha$ emission for the few stars that lack Spitzer data.

3. Based on our classifications, the disk fraction in Taurus, N(II)/N(II+III), is $\sim 75 \%$ for solar-mass stars and declines to $\sim 45 \%$ for low-mass stars and brown dwarfs $\left(0.01-0.3 M_{\odot}\right)$. A similar dependence on stellar mass has been observed in Chamaeleon I (Luhman et al. 2008a). In contrast to Taurus and Chamaeleon I, IC 348 exhibits a disk fraction of only $\sim 20 \%$ for solarmass stars (Lada et al. 2006; Muench et al. 2007; Luhman et al. 2005). Given that IC 348 is roughly coeval with Chamaeleon I ( $\tau \sim 2-3 \mathrm{Myr})$, the comparison of these three regions suggests that disk lifetimes for solar-mass stars are longer in starforming regions like Taurus and Chamaeleon I that have lower stellar densities.

4. As previously observed in Taurus (Hartmann 2002), we find that the positions of the class I and II members closely follow the distribution of 
dense gas while the class III stars are more widely distributed. An analysis of the nearest neighbor distances also suggests that class II sources may have a wider distribution than class I sources, although this difference is only marginally significant. The median of the nearest neighbor distances for classes I and II is $0.15 \mathrm{pc}$, which is twice the average value in nearby star-forming clusters (Gutermuth et al. 2009).

5. Our study has produced multiple epochs of mid-IR photometry for $\sim 200$ members of Taurus. In these data, the mid-IR variability of class I/II sources is much greater than that of class III stars, which agrees with similar measurements with Spitzer in Chamaeleon I (Luhman et al. 2008a). The fraction of disk-bearing stars that are variable is higher in Taurus than in Chamaeleon I, indicating that the variability of disks decreases with age.

6. We have used our Spitzer photometry for the disk population in Taurus to refine the observational criteria for the evolutionary phases of disks. When plotted in terms of $K_{s}-[5.8], K_{s}-$ [8.0], and $K_{s}-[24]$, the members of Taurus appear predominantly within two distinct groups that are wellseparated from each other, as found in early midIR studies of Taurus (Skrutskie et al. 1990). The colors of the stars in the bluer group are consistent with stellar photospheres. We have defined the large, continuous population of much redder sources as primordial disks. Using our models of accretion disks, we have demonstrated that the colors of these primordial disks can be explained in terms of optically thick disks. The sources that fall within the gap between primordial disks and stellar photospheres are defined as evolved disks (weak excess in all bands), transitional disks (weak or no excess at $\lambda<10 \mu \mathrm{m}$, large excess at longer $\lambda$ ), and evolved transitional disks or debris disks (only weak $24 \mu \mathrm{m}$ excess). We have identified 19 members of
Taurus that are candidates for disks in these later stages of disk evolution, 11 of which have not been previously recognized as such.

7. We have applied our classification criteria for disks to nearby clusters and associations with ages of 2 10 Myr that have been observed with Spitzer. We find that the number of evolved and transitional disks in those regions is consistent with the paucity of such disks in Taurus. Some of the sources that have been classified as evolved and transitional disks in previous Spitzer studies have colors that are similar to those of the bluer primordial disks in Taurus (i.e., flatter optically thick disks). The number ratio of evolved and transitional disks to primordial disks in Taurus is 15/98 for spectral types of K5-M5, indicating a timescale of inner disk clearing that is $\sim 15 \%$ of the lifetime of primordial disks $(\sim 3 \mathrm{Myr})$. The data in Taurus and the older populations that we have examined are inconsistent with notion that the lifetime of the evolved and transitional phases (i.e., disk clearing timescale) is comparable to that of primordial disks.

K. L. was supported by grant AST-0544588 from the National Science Foundation. C. E. and N. C. were supported by grant NNX08AH94G from NASA and grant 1344183 from the Jet Propulsion Laboratory. We thank Dan Watson and Melissa McClure for their analysis of unpublished IRS spectra. We are grateful to Jesus Hernández for helpful comments. This work makes use of data from the Spitzer Space Telescope and 2MASS. Spitzer is operated by the Jet Propulsion Laboratory, California Institute of Technology under a contract with NASA. 2MASS is a joint project of the University of Massachusetts and the Infrared Processing and Analysis Center/California Institute of Technology, funded by NASA and the NSF. The Center for Exoplanets and Habitable Worlds is supported by the Pennsylvania State University, the Eberly College of Science, and the Pennsylvania Space Grant Consortium.

\section{APPENDIX}

\section{INFRARED COLORS OF YOUNG STELLAR PHOTOSPHERES}

We have estimated the intrinsic IR colors of young stellar photospheres as a function of spectral type from K4 to L0 $\left(M \sim 0.01-1 M_{\odot}\right)$. For this analysis, we examined the colors of probable members of the $\eta$ Cha, $\epsilon$ Cha, and TW Hya associations (TWA, Mamaiek et al. 1999; Webb et al. 1999; Lawson et al. 2002; Gizis 2002; Feigelson et al. 2003; Luhman \& Steeghs 2004; Luhman|2004a; Lyo et al.|2004; Song et al.|2004; Zuckerman \& Song 2004; Mamajek 2005; Scholz et al. 2005; Looper et al. 2007; Luhman et al.|2008a; Kastner et al. 2008) and young late-type dwarfs in the solar neighborhood (Kirkpatrick et al. 2006, 2008; Cruz et al. 2007, 2009). These sources should have negligible extinction $\left(A_{V}<1\right)$ since they are relatively nearby $(d \lesssim 100 \mathrm{pc})$ and are not associated with molecular clouds. As a result, their observed colors should not depart from the intrinsic photospheric values unless emission from circumstellar disks is present. Although the members of embedded clusters are subject to both reddening and excess emission from disks, the bluest sources provide useful constraints on the intrinsic colors of photospheres. Taurus and Chamaeleon I are the best star-forming regions for this purpose since accurate spectral types and Spitzer photometry have been measured for most of their members and the census of each population is large and reaches the end of the M spectral sequence (Luhman 2007; Luhman \& Muench 2008; Luhman et al. 2009b, references therein).

We have adopted measurements of $J, H$, and $K_{s}$ from the 2MASS Point Source Catalog when they are available. For the faintest members of Chamaeleon I, we used photometry measured from deeper near-IR images that were calibrated with 2MASS sources (Luhman 2007). In the Spitzer bands between 3.6 and $24 \mu$ m, we have used our photometry in Taurus and our previous measurements for Chamaeleon I (Luhman et al. 2008a; Luhman \& Muench 2008), $\epsilon$ Cha (Luhman et al. 2008a), and young late-type dwarfs (Luhman et al. 2009b). We have analyzed all Spitzer images of $\eta$ Cha, TWA, and additional members of $\epsilon$ Cha with the same methods that we have applied to Taurus. The resulting photometry for the members of these associations is presented in Tables 9] 12, Stars that were not observed by either 
IRAC or MIPS are excluded from these tabulations.

The IR colors for Taurus, Chamaeleon I, $\eta$ Cha, $\epsilon$ Cha, TWA, and young dwarfs are plotted as a function of spectral type in Figure 26. The limits of these diagrams encompass only the bluest colors since we wish to examine the colors of the stellar photospheres. As a result, many of the stars with disks are too red to appear in Figure 26. particularly in the colors at longer wavelengths. The spectral types that we have measured in TWA from unpublished spectra are a few subclasses later than some of the classifications from previous studies. Therefore, we have plotted only the TWA members for which we have measured types, or that have been classified with similar methods (Looper et al. 2007; Herczeg et al. 2009). We also have excluded measurements that have photometric uncertainties greater than 0.1 mag. Few of the class III sources at the latest types have accurate $24 \mu \mathrm{m}$ data. To help constrain the photospheric colors at those types, we have measured IRAC and MIPS photometry for field dwarfs from M9-L0 and have included them in the diagram for [8.0] - [24]. These stars consist of BRI 0021-0214, LHS 2065, LP944-20, LHS 2924, and 2MASS J07464256+2000321. The [8.0] - [24] color of the TWA brown dwarf 2MASSW J1139511-315921 (M8.5) is similar to colors of these fields dwarfs, which illustrates the absence of $24 \mu \mathrm{m}$ excess emission from this object that was discussed by Morrow et al. (2008).

For each IR color, we have estimated the photospheric values as a function of spectral type by performing a fit to the sequence of class III objects from the young associations and the solar neighborhood. This fit was also constrained to agree with the blue envelope of colors from Taurus and Chamaeleon I. These color relations are plotted in Figure 26 and are presented in Table 13.

\section{REFERENCES}

Adams, F. C., Lada, C. J., \& Shu, F. H. 1987, ApJ, 571, 378

Allen, L. E., et al. 2004, ApJS, 154, 363

André, P., Motte, F., Bacmann, A. 1999, ApJ, 513, L57

André, P., Ward-Thompson, D., \& Barsony, M. 1993, ApJ, 406, 122

Andrews, S. M., \& Williams, J. P. 2005, ApJ, 631, 1134

Backman, D., \& Paresce, F. 1993, Protostars and Planets III, ed. E. H. Levy, J. I. Lunine (Tucson, AZ: Univ. Arizona Press), 1253

Balona, L. A., \& Laney, C. D. 1996, MNRAS, 281, 1341

Baraffe, I., Chabrier, G., Allard, F., \& Hauschildt, P. H. 1998, A\&A, 337, 403

Barrado y Navascués, D., et al. 2007, ApJ, 664, 481

Beck, T. L. 2007, AJ, 133, 1673

Beichman, C. A., Myers, P. C., Emerson, J. P., Harris, S., Mathieu, R., Benson, P. J., \& Jennings, R. E. 1986, ApJ, 307, 337

Bontemps, S., André, P., Terebey, S., \& Cabrit, S. 1996, A\&A, 311,858

Bourke, T. L., et al. 2006, ApJ, 649, L37

Briceño, C., Calvet, N., Hernández, J., Vivas, A. K., Hartmann, L., Downes, J. J., \& Berlind P. 2005, AJ, 129, 907

Briceño, C., Hartmann, L., Hernandez, J., Calvet, N., Katherina Vivas, A., Furesz, G., \& Szentgyorgyi, A. 2007, ApJ, 661, 1119

Briceño, C., Hartmann, L., Stauffer, J., \& Martín, E. L., 1998, AJ, 115, 2074

Briceño, C., Luhman, K. L., Hartmann, L., Stauffer, J. R., \& Kirkpatrick, J. D. 2002, ApJ, 580, 317

Brown, J. M., Blake, G. A., Qi, C., Dullemond, C. P., \& Wilner, D. J. 2008, ApJ, 675, L109

Burgasser, A. J., Kirkpatrick, J. D., Reid, I. N., Brown, M. E., Miskey, C. L., \& Gizis, J. E. 2003, ApJ, 586, 512

Calvet, N., et al. 2005, ApJ, 630, L185

Carpenter, J. M., Mamajek, E. E., Hillenbrand, L. A., \& Meyer, M. R. 2006, ApJ, 651, L49

Carpenter, J. M., Mamajek, E. E., Hillenbrand, L. A., \& Meyer, M. R. 2009, ApJ, 705, 1646

Chabrier, G., Baraffe, I., Allard, F., \& Hauschildt, P. 2000, ApJ, 542, L119

Chen, H., Myers, P. C., Ladd, E. F., \& Wood, D. O. S. 1995, ApJ, 445, 377

Covino, E., Alcalá, J. M., Allain, S., Bouvier, J., Terranegra, L., \& Krautter, J. 1997, A\&A, 328, 187

Cowley, A. 1972, AJ, 77, 750

Cruz, K. L., Kirkpatrick, J. D., \& Burgasser, A. J. 2009, AJ, 137, 3345

Cruz, K. L., et al. 2007, AJ, 133, 439

Currie, T., \& Kenyon, S. J. 2009b, AJ, 138, 703

Currie, T., Kenyon, S. J., Balog, Z., Rieke, G., Bragg, A., \& Bromley, B. 2008, ApJ, 672, 558

Currie, T., Lada, C. J., Plavchan, P., Robitaille, T. P., Irwin, J., \& Kenyon, S. J. 2009a, ApJ, 698, 1
Dahm, S. E. 2005, AJ, 130, 1805

Dahm, S. E., \& Hillenbrand, L. A. 2007, AJ, 133, 2072

D'Alessio, P., Calvet, N., Hartmann, L., Franco-Hernández, R., \& Servín, H. 2006, ApJ, 638, 314

D'Alessio, P., et al. 2005, ApJ, 621, 461

Damjanov, I., Jayawardhana, R., Scholz, A., Ahmic, M., Nguyen, D. C., et al. 2007, ApJ, 670, 1337

Dobashi, K., Uehara, H., Kandori, R., Sakurai, T., Kaiden, M., Umemoto, T., \& Sato, F. 2005, PASJ, 57, 1

Downes, J. J., Briceño, C., Hernández, J., Calvet, N., Hartmann, L., \& Balaguer, E. P. 2008, AJ, 136, 51

Draine, B. T. \& Lee, H. M. 1984, ApJ, 285, 89

Duchêne, G., Bouvier, J., Bontemps, S., André, P., \& Motte, F. 2004, A\&A, 427, 651

Duchêne, G., Monin, J.-L., Bouvier, J., \& Ménard, F. 1999, A\&A, 351,954

Dunham, M. M., et al. 2006, ApJ, 651, 945

Dutrey, A., et al. 2008, A\&A, 490, L15

Engelbracht, C. W., et al. 2007, PASP, 119, 994

Ercolano, B., Clarke, C. J., \& Robitaille, T. P. 2009, MNRAS, 394, L141

Espaillat, C., Calvet, N., Luhman, K. L., Muzerolle, J., \& D'Alessio, P. 2008a, ApJ, 682, L125

Espaillat, C., et al. 2007a, ApJ, 664, L111

Espaillat, C., et al. 2007b, ApJ, 670, L135

Espaillat, C., et al. 2008b, ApJ, 689, L145

Evans, N. J., II, et al. 2009, ApJS, 181, 321

Fazio, G. G., et al. 2004, ApJS, 154, 10

Feigelson, E. D., Lawson, W. A., \& Garmire, G. P. 2003, ApJ, 599,1207

Flaherty, K. M., Pipher, J. L., Megeath, S. T., Winston, E. M., Gutermuth, R. A., Muzerolle, J., Allen, L. E., \& Fazio, G. G. 2007, ApJ, 663, 1069

Forrest, W. J., et al. 2004, ApJS, 154, 443

Furlan, E., et al. 2006, ApJS, 165, 568

Furlan, E., et al. 2008, ApJS, 176, 184

Furlan, E., et al. 2009, ApJ, 706, 1194

Gautier, T. N., III, Rebull, L. M., Stapelfeldt, K. R., \& Mainzer, A. 2008, ApJ, 683, 813

Gizis, J. E. 2002, ApJ, 575, 484

Greene, T. P., Wilking, B. A., André, P., Young, E. T., \& Lada, C. J. 1994, ApJ, 434, 614

Güdel, M., et al. 2007, A\&A, 468, 353

Guenther, E., Esposito, M., Mundt, R., Covino, E., Alcalá, J. M., et al. 2007, A\&A, 467, 1147

Guieu, S., Dougados, C., Monin, J.-L., Magnier, E. \& Martín, E. L. 2006, A\&A, 446, 485

Guieu, S., et al. 2007, A\&A, 465, 855

Gutermuth, R. A., et al. 2008, ApJ, 674, 336

Gutermuth, R. A., et al. 2009, ApJS, 184, 18

Haisch, K. E., Lada, E. A., \& Lada, C. J. 2001, ApJ, 553, L153

Hartigan, P., \& Kenyon, S. J. 2003, ApJ, 583, 334 
Hartigan, P., Strom, K. M., \& Strom, S. E. 1994, ApJ, 427, 961 Hartmann, L. 2002, ApJ, 578, 914

Hartmann, L., Megeath, S. T., Allen, L., Luhman, K., Calvet, N., D'Alessio, P., Franco-Hernandez, R., \& Fazio, G. 2005, ApJ, 629,881

Herczeg, G. J., Cruz, K. L., \& Hillenbrand, L. A. 2009, ApJ, 696, 1589

Hernández, J., Briceño, C., Calvet, N., Hartmann, L., Muzerolle, J., \& Quintero, A. 2006, ApJ, 652, 472

Hernández, J., et al. 2007a, ApJ, 662, 1067

Hernández, J., et al. 2007b, ApJ, 671, 1784

Hernández, J., Hartmann, L., Calvet, N., Jeffries, R. D., Gutermuth, R., Muzerolle, J., \& Stauffer, J. 2008, ApJ, 686, 1195

Hughes, A. M., Wilner, D. J., Calvet, N., D'Alessio, P., Claussen, M. J., \& Hogerheijde, M. R. 2007, ApJ, 664, 536

Hughes, A. M., et al. 2009, ApJ, 698, 131

Ireland, M. J., \& Kraus, A. L. 2008, ApJ, 678, L59

Irwin, J., et al. 2008, MNRAS, 384, 675

Kastner, J. H., Zuckerman, B., \& Bessell, M. 2008, A\&A, 491, 829

Kenyon, S. J., \& Bromley, B. C. 2005, ApJ, 130, 269

Kenyon, S. J., Brown, D. I., Tout, C. A., \& Berlind, P. 1998, AJ, 115,2491

Kenyon, S. J., Gómez, M., Marzke, R. O., \& Hartmann, L. 1994, AJ, 108, 251

Kenyon, S. J., Gómez, M., \& Whitney, B. A. 2008, in Handbook of Star Forming Regions, Vol. 1, The Northern Sky, ASP Monograph Series 4, ed. B. Reipurth (San Francisco, CA: ASP), 405

Kenyon, S. J., \& Hartmann, L. 1995, ApJS, 101, 117

Kenyon, S. J., Hartmann, L. W., Strom, K. M., \& Strom, S. E. 1990, AJ, 99, 869

Kim, K. H., et al. 2009, ApJ, 700, 1017

Kirkpatrick, J. D., et al. 2006, ApJ, 639, 1120

Kirkpatrick, J. D., et al. 2008, ApJ, 689, 1295

Kraus, A. L., White, R. J., \& Hillenbrand, L. A. 2006, ApJ, 649, 306

Lada, C. J. 1987, in IAU Symp. 115, Star Forming Regions, ed. M. Peimbert \& J. Jugaku (Dordrecht: Reidel), 1

Lada, C. J., \& Wilking, B. A. 1984, ApJ, 287, 610

Lada, C. J., et al. 2006, AJ, 131, 1574

Lawson, W. A., Crause, L. A., Mamajek, E. E., \& Feigelson, E. D. 2001, MNRAS, 321, 57

Lawson, W. A., Crause, L. A., Mamajek, E. E., \& Feigelson, E. D. 2002, MNRAS, 329, L29

Looper, D. L., Burgasser, A. J., Kirkpatrick, J. D., \& Swift, B. J. 2007, ApJ, 669, L97

Luhman, K. L. 2004a, ApJ, 616, 1033

Luhman, K. L. 2004b, ApJ, 617, 1216

Luhman, K. L. 2006, ApJ, 645, 676

Luhman, K. L. 2007, ApJS, 173, 104

Luhman, K. L., Briceño, C., Stauffer, J. R., Hartmann, L., Barrado y Navascués, D., \& Nelson, C. 2003a, ApJ, 590, 348

Luhman, K. L., Hernández, J., Downes, J. J., Hartmann, L., \& Briceño, C. 2008b, ApJ, 688, 362

Luhman, K. L., Joergens, V., Lada, C., Muzerolle, J., Pascucci, I., \& White, R. 2007b, Protostars and Planets V, B. Reipurth, D. Jewitt, and K. Keil (eds.), University of Arizona Press, Tucson, 443

Luhman, K. L., Mamajek, E. E., Allen, P. R., Muench, A. A., \& Finkbeiner, D. P. 2009a, ApJ, 691, 1265

Luhman, K. L., Mamajek, E. E., Cruz, K. L., \& Allen, P. R. 2009b, ApJ, 703, 399

Luhman, K. L., \& Muench, A. A. 2008, ApJ, 684, 654

Luhman, K. L., \& Rieke, G. H. 1998, ApJ, 497, 354

Luhman, K. L., Stauffer, J. R., Muench, A. A., Rieke, G. H., Lada, E. A., Bouvier, J., \& Lada, C. J. 2003b, ApJ, 593, 1093

Luhman, K. L., \& Steeghs, D. 2004, ApJ, 609, 917

Luhman, K. L., Whitney, B. A., Meade, M. R., Babler, B. L., Indebetouw, R., Bracker, S., \& Churchwell, E. B. 2006, ApJ, 647,1180

Luhman, K. L., et al. 2005, ApJ, 631, L69

Luhman, K. L., et al. 2007a, ApJ, 666, 1219

Luhman, K. L., et al. 2008a, ApJ, 675, 1375

Lyo, A.-R, Lawson, W. A., Feigelson, E. D., \& Crause, L. A. 2004, MNRAS, 347, 246

Malfait, K., Bogaert, E., \& Waelkens, C. 1998, A\&A, 331, 211
Mamajek, E. E. 2005, ApJ, 634, 1385

Mamajek, E., Lawson, W. A., \& Feigelson, E. D. 1999, ApJ, 516, 77

Mannings, V., Koerner, D. W., \& Sargent, A. J. 1997, Nature, 388,555

Mayne, N. J., \& Naylor, T. 2008, MNRAS, 386, 261

Marengo, M., Megeath, S. T., Fazio, G. G., Stapelfeldt, K. R.,

Werner, M. W., Backman, D. E. 2006, ApJ, 647, 1437

Martín, E. L. 2000, AJ, 120, 2114

Martín, E. L., Dougados, C., Magnier, E., Ménard, F., Magazzù, A., Cuilandre, J.-C., \& Delfosse, X. 2001, ApJ, 561, L195

Massarotti, A., Latham, D. W., Torres, G., Brown, R. A., \& Oppenheimer, B. D. 2005, AJ, 129, 2294

Megeath, S. T., Hartmann, L., Luhman, K. L., \& Fazio, G. G. 2005, ApJ, 634, L113

Megeath, S. T., et al. 2004, ApJS, 154, 367

Moitinho, A., Alves, J., Huelamo, N., \& Lada, C. J. 2001, ApJ, $563, \mathrm{~L} 73$

Moriarty-Schieven, G. H., Butner, H. M., \& Wannier, P. G. 1995, ApJ, 445, L55

Moriarty-Schieven, G. H., Wannier, P. G., Tamura, M., \& Keene, J. 1992, ApJ, 400, 260

Morrow, A. L., et al. 2008, ApJ, 676, L143

Motte, F. 1998, Ph.D. Thesis, Univeristy Paris XI

Motte, F., \& André, P. 2001, A\&A, 365, 440

Motte, F., André, P., \& Neri, R. 1998, A\&A, 336, 150

Muench, A. A., Lada, C. J., Luhman, K. L., Muzerolle, J., \& Young, E. 2007, AJ, 134, 411

Muzerolle, J., Hillenbrand, L., Calvet, N., Briceño, C., \&

Hartmann, L. 2003, ApJ, 592, 266

Najita, J. R., Strom, S. E., \& Muzerolle, J. 2007, MNRAS, 378, 369

Pozzo, M., Jeffries, R. D., Naylor, T., Totten, E. J., Harmer, S., \& Kenyon, M. 2000, MNRAS, 313, L23

Prato, L., Lockhart, K. E., Johns-Krull, C. M., \& Rayner, J. T. 2009, AJ, 137, 3931

Preibisch, T., Brown, A. G. A., Bridges, T., Guenther, E., \& Zinnecker, H., 2002, AJ, 124, 404

Preibisch, T., \& Mamajek, E. 2008, in Handbook of Star Forming Regions, Vol. 2, The Southern Sky, ASP Monograph Series 5, ed. B. Reipurth (San Francisco, CA: ASP), 235

Prusti, T., Clark, F. O., Laureijs, R. J., Wakker, B. P., \& Wesselius, P. R. 1992, A\&A, 259, 537

Quillen, A. C., Blackman, E. G., Frank, A., \& Varni'ere, P., 2004, ApJ, 612, L137

Rebull, L. M., et al. 2010, ApJS, in press

Reach, W. T., et al. 2005, PASP, 117, 978

Rice, W. K. M., Wood, K., Armitage, P. J., Whitney, B. A., \& Bjorkman, J. E. 2003, MNRAS, 342, 79

Rieke, G. H., \& Lebofsky, M. J. 1985, ApJ, 288, 618

Rieke, G. H., et al. 2004, ApJS, 154, 25

Rieke, G. H., et al. 2005, ApJ, 620, 1010

Scholz, R.-D., McCaughrean, M. J., Zinnecker, H., \& Lodieu, N. 2005, A\&A, 430, L49

Skrutskie, M. F., Dutkevitch, D., Strom, S. E., Edwards, S., Strom, K. M., \& Shure, M. A. 1990, AJ, 99, 1187

Skrutskie, M., et al. 2006, AJ, 131, 1163

Slesnick, C. L., Carpenter, J. M., Hillenbrand, L. A., \& Mamajek, E. E. 2006, AJ, 132, 2665

Sicilia-Aguilar, A., Hartmann, L. W., Hernández, J., Briceño, C., \& Calvet, N. 2005, AJ, 130, 188

Sicilia-Aguilar, A., et al. 2006, ApJ, 638, 897

Sicilia-Aguilar, A., et al. 2009, ApJ, 701, 1188

Simon, M., \& Prato, L. 1995, ApJ, 450, 824

Song, I., Zuckerman, B., \& Bessell, M. S. 2004, ApJ, 600, 1016

Steffen, A. T., et al. 2001, AJ, 122, 997

Strom, K. M., \& Strom, S. E. 1994, ApJ, 424, 237

Strom, K. M., Strom, S. E., Edwards, S., Cabrit, S., \& Skrutskie, M. F. 1989, AJ, 97, 1451

Takami, M., Bailey, J., \& Chrysostomou, A. 2003, A\&A, 397, 675

Tamura, M., Gatley, I., Waller, W., \& Werner, M. W. 1991, ApJ, 374, L25

Torres, C. A. O., Quast, G., de La Reza, R., Gregorio-Hetem, J., Lepine, J. R. D. 1995, AJ, 109, 2146

Walter, F. M., Beck, T. L., Morse, J. A., \& Wolk, S. J. 2003, AJ, 125,2123

Watson, D. M., et al. 2004, ApJS, 154, 391 
Webb, R. A., Zuckerman, B., Platais, I., Patience, J., White, R. J., Schwartz, M. J., \& McCarthy, C. 1999, ApJ, 512, L63

Werner, M. W., et al. 2004, ApJS, 154, 1

White, R. J., \& Basri, G. 2003, ApJ, 582, 1109

White, R. J., \& Ghez, A. M. 2001, ApJ, 556, 265

White, R. J., Ghez, A. M., Reid, I. N., \& Schultz, G. 1999, ApJ, 520,811
Wichmann, R., et al. 1996, A\&A, 312, 439

Wolk, S. J., \& Walter, F. M. 1996, AJ, 111, 2066

Zasowski, G., Kemper, F., Watson, D. M., Furlan, E., Bohac, C. J., Hull, C., \& Green, J. D. 2009, ApJ, 694, 459

Zuckerman, B., \& Song, I. 2004, ARA\&A, 42, 685 
TABLE 1

IRAC OBSERVING LOG

\begin{tabular}{|c|c|c|c|c|c|c|c|}
\hline AOR & PID & $\begin{array}{l}\text { Date } \\
\text { (UT) }\end{array}$ & $\begin{array}{c}\text { 3.6/5.8 Center } \\
(\mathrm{J} 2000)\end{array}$ & $\begin{array}{c}\text { 4.5/8.0 Center } \\
(\mathrm{J} 2000)\end{array}$ & $\begin{array}{l}\text { Dimensions } \\
(\operatorname{arcmin})\end{array}$ & $\begin{array}{c}\text { Angle }^{\mathrm{a}} \\
(\mathrm{deg})\end{array}$ & $\operatorname{Exp}_{(\mathrm{s})} \operatorname{Time}^{\mathrm{b}}$ \\
\hline \multirow[t]{20}{*}{3963904} & 37 & 2004 Feb 9 & $044642.3+245858$ & $044642.3+245858$ & $5.3 \times 5.3$ & 85.0 & $31.2 \mathrm{HDR}$ \\
\hline & & & $044646.6+245221$ & $044638.5+250543$ & $5.3 \times 5.3$ & 85.0 & $31.2 \mathrm{HDR}$ \\
\hline & & & $044431.0+251221$ & $044431.0+251221$ & $5.3 \times 5.3$ & 85.0 & $31.2 \mathrm{HDR}$ \\
\hline & & & $044434.9+250550$ & $044427.2+251906$ & $5.3 \times 5.3$ & 85.0 & $31.2 \mathrm{HDR}$ \\
\hline & & & $044303.4+252010$ & $044303.4+252010$ & $5.3 \times 5.3$ & 85.0 & $31.2 \mathrm{HDR}$ \\
\hline & & & $044307.2+251344$ & $044300.0+252656$ & $5.3 \times 5.3$ & 85.0 & $31.2 \mathrm{HDR}$ \\
\hline & & & $044238.0+251529$ & $044238.0+251529$ & $5.3 \times 5.3$ & 85.0 & $31.2 \mathrm{HDR}$ \\
\hline & & & $044241.4+250854$ & $044234.1+252214$ & $5.3 \times 5.3$ & 85.0 & $31.2 \mathrm{HDR}$ \\
\hline & & & $044221.5+252026$ & $044221.5+252026$ & $5.3 \times 5.3$ & 85.0 & $31.2 \mathrm{HDR}$ \\
\hline & & & $044224.8+251353$ & $044217.7+252711$ & $5.3 \times 5.3$ & 85.0 & $31.2 \mathrm{HDR}$ \\
\hline & & & $044207.6+252253$ & $044207.6+252253$ & $5.3 \times 5.3$ & 85.0 & $31.2 \mathrm{HDR}$ \\
\hline & & & $044211.2+251621$ & $044203.8+252938$ & $5.3 \times 5.3$ & 85.0 & $31.2 \mathrm{HDR}$ \\
\hline & & & $044104.5+245102$ & $044104.5+245102$ & $5.3 \times 5.3$ & 85.0 & $31.2 \mathrm{HDR}$ \\
\hline & & & $044108.7+244422$ & $044100.5+245746$ & $5.3 \times 5.3$ & 85.0 & $31.2 \mathrm{HDR}$ \\
\hline & & & $044139.7+255617$ & $044139.7+255617$ & $5.3 \times 5.3$ & 85.0 & $31.2 \mathrm{HDR}$ \\
\hline & & & $044142.4+254948$ & $044135.9+260301$ & $5.3 \times 5.3$ & 85.0 & $31.2 \mathrm{HDR}$ \\
\hline & & & $044112.8+254638$ & $044112.8+254638$ & $5.3 \times 5.3$ & 85.0 & $31.2 \mathrm{HDR}$ \\
\hline & & & $044117.0+253951$ & $044109.0+255322$ & $5.3 \times 5.3$ & 85.0 & $31.2 \mathrm{HDR}$ \\
\hline & & & $044050.0+255118$ & $044050.0+255118$ & $5.3 \times 5.3$ & 85.0 & $31.2 \mathrm{HDR}$ \\
\hline & & & $044053.4+254436$ & $044046.1+255802$ & $5.3 \times 5.3$ & 85.0 & $31.2 \mathrm{HDR}$ \\
\hline \multirow[t]{6}{*}{3965440} & 37 & 2004 Feb 9 & $044749.0+292509$ & $044749.0+292509$ & $5.3 \times 5.3$ & 87.0 & $31.2 \mathrm{HDR}$ \\
\hline & & & $044751.2+291826$ & $044746.6+293156$ & $5.3 \times 5.3$ & 87.0 & $31.2 \mathrm{HDR}$ \\
\hline & & & $044320.4+294008$ & $044320.4+294008$ & $5.3 \times 5.3$ & 87.0 & $31.2 \mathrm{HDR}$ \\
\hline & & & $044323.6+293319$ & $044317.6+294654$ & $5.3 \times 5.3$ & 87.0 & $31.2 \mathrm{HDR}$ \\
\hline & & & $044117.7+283950$ & $044117.7+283950$ & $5.3 \times 5.3$ & 87.0 & $31.2 \mathrm{HDR}$ \\
\hline & & & $044119.7+283318$ & $0441 \quad 14.5+284637$ & $5.3 \times 5.3$ & 87.0 & $31.2 \mathrm{HDR}$ \\
\hline \multirow{2}{*}{3967488} & 37 & 2004 Feb 10 & $041447.7+264722$ & $041447.7+264722$ & $5.3 \times 5.3$ & 82.0 & $31.2 \mathrm{HDR}$ \\
\hline & & & $041453.2+264038$ & $041442.6+265405$ & $5.3 \times 5.3$ & 82.0 & $31.2 \mathrm{HDR}$ \\
\hline \multirow[t]{2}{*}{3967232} & 37 & 2004 Feb 11 & $040439.4+215822$ & $040439.4+215822$ & $5.3 \times 5.3$ & 80.0 & $31.2 \mathrm{HDR}$ \\
\hline & & & $040445.9+215141$ & $040433.4+220500$ & $5.3 \times 5.3$ & 80.0 & $31.2 \mathrm{HDR}$ \\
\hline \multirow[t]{2}{*}{3966464} & 37 & 2004 Feb 12 & $050749.7+302407$ & $050749.7+302407$ & $5.3 \times 5.3$ & 89.5 & $31.2 \mathrm{HDR}$ \\
\hline & & & $050751.3+301717$ & $050748.5+303055$ & $5.3 \times 5.3$ & 89.5 & $31.2 \mathrm{HDR}$ \\
\hline \multirow[t]{12}{*}{3964928} & 37 & 2004 Feb 14 & $050755.1+250003$ & $050755.1+250003$ & $5.3 \times 5.3$ & 86.0 & $31.2 \mathrm{HDR}$ \\
\hline & & & $050757.7+245331$ & $050752.4+250651$ & $5.3 \times 5.3$ & 86.0 & $31.2 \mathrm{HDR}$ \\
\hline & & & $0507 \quad 12.4+243708$ & $050712.4+243708$ & $5.3 \times 5.3$ & 86.0 & $31.2 \mathrm{HDR}$ \\
\hline & & & $050715.2+243040$ & $050709.5+244354$ & $5.3 \times 5.3$ & 86.0 & $31.2 \mathrm{HDR}$ \\
\hline & & & $050623.8+243217$ & $050623.8+243217$ & $5.3 \times 5.3$ & 86.0 & $31.2 \mathrm{HDR}$ \\
\hline & & & $050626.4+242535$ & $050620.9+243904$ & $5.3 \times 5.3$ & 86.0 & $31.2 \mathrm{HDR}$ \\
\hline & & & $050617.3+244602$ & $050617.3+244602$ & $5.3 \times 5.3$ & 86.0 & $31.2 \mathrm{HDR}$ \\
\hline & & & $050619.6+243927$ & $050614.4+245248$ & $5.3 \times 5.3$ & 86.0 & $31.2 \mathrm{HDR}$ \\
\hline & & & $050523.0+253134$ & $050523.0+253134$ & $5.3 \times 5.3$ & 86.0 & $31.2 \mathrm{HDR}$ \\
\hline & & & $050526.1+252445$ & $050520.3+253821$ & $5.3 \times 5.3$ & 86.0 & $31.2 \mathrm{HDR}$ \\
\hline & & & $050442.2+250944$ & $050442.2+250944$ & $5.3 \times 5.3$ & 86.0 & $31.2 \mathrm{HDR}$ \\
\hline & & & $050444.0+250313$ & $050439.4+251630$ & $5.3 \times 5.3$ & 86.0 & $31.2 \mathrm{HDR}$ \\
\hline \multirow[t]{14}{*}{3965696} & 37 & 2004 Feb 14 & $045600.0+303350$ & $045600.0+303350$ & $5.3 \times 5.3$ & 88.0 & $31.2 \mathrm{HDR}$ \\
\hline & & & $045601.5+302717$ & $045557.6+304037$ & $5.3 \times 5.3$ & 88.0 & $31.2 \mathrm{HDR}$ \\
\hline & & & $045546.3+303303$ & $045546.3+303303$ & $5.3 \times 5.3$ & 88.0 & $31.2 \mathrm{HDR}$ \\
\hline & & & $045547.9+302619$ & $045544.1+303951$ & $5.3 \times 5.3$ & 88.0 & $31.2 \mathrm{HDR}$ \\
\hline & & & $045602.3+302056$ & $045602.3+302056$ & $5.3 \times 5.3$ & 88.0 & $31.2 \mathrm{HDR}$ \\
\hline & & & $045603.9+301419$ & $045600.2+302744$ & $5.3 \times 5.3$ & 88.0 & $31.2 \mathrm{HDR}$ \\
\hline & & & $045537.3+301747$ & $045537.3+301747$ & $5.3 \times 5.3$ & 88.0 & $31.2 \mathrm{HDR}$ \\
\hline & & & $045539.6+301118$ & $045535.2+302435$ & $5.3 \times 5.3$ & 88.0 & $31.2 \mathrm{HDR}$ \\
\hline & & & $045511.5+302150$ & $045511.5+302150$ & $5.3 \times 5.3$ & 88.0 & $31.2 \mathrm{HDR}$ \\
\hline & & & $045513.2+301515$ & $045509.4+302838$ & $5.3 \times 5.3$ & 88.0 & $31.2 \mathrm{HDR}$ \\
\hline & & & $045206.7+304720$ & $045206.7+304720$ & $5.3 \times 5.3$ & 88.0 & $31.2 \mathrm{HDR}$ \\
\hline & & & $045209.3+304029$ & $045204.6+305407$ & $5.3 \times 5.3$ & 88.0 & $31.2 \mathrm{HDR}$ \\
\hline & & & $045148.0+304703$ & $045148.0+304703$ & $5.3 \times 5.3$ & 88.0 & $31.2 \mathrm{HDR}$ \\
\hline & & & $045149.5+304031$ & $045145.8+305350$ & $5.3 \times 5.3$ & 88.0 & $31.2 \mathrm{HDR}$ \\
\hline 4915200 & 94 & 2004 Feb 14 & $045503.5+303126$ & $045501.3+303813$ & $14.3 \times 19.0$ & 177.5 & $52 \mathrm{HDR}$ \\
\hline 4916992 & 94 & 2004 Feb 14 & $050424.7+250816$ & $050421.9+251502$ & $10.3 \times 15.0$ & 176.0 & $52 \mathrm{HDR}$ \\
\hline 4915456 & 94 & 2004 Feb 15 & $045503.5+303126$ & $045501.3+303813$ & $14.3 \times 19.0$ & 177.5 & $52 \mathrm{HDR}$ \\
\hline 4917248 & 94 & 2004 Feb 16 & $050424.7+250816$ & $050421.9+251502$ & $10.3 \times 15.0$ & 176.0 & $52 \mathrm{HDR}$ \\
\hline \multirow[t]{2}{*}{3966720} & 37 & 2004 Mar 6 & $044659.6+170042$ & $044659.6+170042$ & $5.3 \times 5.3$ & 83.5 & $31.2 \mathrm{HDR}$ \\
\hline & & & $044704.0+165356$ & $044655.6+170727$ & $5.3 \times 5.3$ & 83.5 & $31.2 \mathrm{HDR}$ \\
\hline 3963392 & 37 & 2004 Mar 7 & $043057.0+255637$ & $043057.0+255637$ & $5.3 \times 5.3$ & 82.0 & $31.2 \mathrm{HDR}$ \\
\hline & & & $043102.2+254957$ & $043051.9+260318$ & $5.3 \times 5.3$ & 82.0 & $31.2 \mathrm{HDR}$ \\
\hline & & & $043044.2+260123$ & $043044.2+260123$ & $5.3 \times 5.3$ & 82.0 & $31.2 \mathrm{HDR}$ \\
\hline & & & $043049.5+255453$ & $043039.2+260805$ & $5.3 \times 5.3$ & 82.0 & $31.2 \mathrm{HDR}$ \\
\hline & & & $043007.4+260823$ & $043007.4+260823$ & $5.3 \times 5.3$ & 82.0 & $31.2 \mathrm{HDR}$ \\
\hline & & & $043012.7+260134$ & $043002.2+261505$ & $5.3 \times 5.3$ & 82.0 & $31.2 \mathrm{HDR}$ \\
\hline & & & $042951.4+260641$ & $042951.4+260641$ & $5.3 \times 5.3$ & 82.0 & $31.2 \mathrm{HDR}$ \\
\hline & & & $042956.7+260002$ & $042946.1+261323$ & $5.3 \times 5.3$ & 82.0 & $31.2 \mathrm{HDR}$ \\
\hline & & & $042930.1+261645$ & $042930.1+261645$ & $5.3 \times 5.3$ & 82.0 & $31.2 \mathrm{HDR}$ \\
\hline & & & $042935.2+261020$ & $042924.9+262326$ & $5.3 \times 5.3$ & 82.0 & $31.2 \mathrm{HDR}$ \\
\hline
\end{tabular}


TABLE 1 - Continued

\begin{tabular}{|c|c|c|c|c|c|c|c|}
\hline AOR & PID & $\begin{array}{l}\text { Date } \\
\text { (UT) }\end{array}$ & $\begin{array}{c}\text { 3.6/5.8 Center } \\
(\mathrm{J} 2000)\end{array}$ & $\begin{array}{c}\text { 4.5/8.0 Center } \\
(\mathrm{J} 2000)\end{array}$ & $\begin{array}{l}\text { Dimensions } \\
\text { (arcmin) }\end{array}$ & $\begin{array}{c}\text { Angle }^{\mathrm{a}} \\
(\mathrm{deg})\end{array}$ & $\operatorname{Exp}_{(\mathrm{s})} \operatorname{Time}^{\mathrm{b}}$ \\
\hline \multirow{50}{*}{3963648} & \multirow{50}{*}{37} & \multirow{50}{*}{2004 Mar 7} & $042944.1+263144$ & $042944.1+263144$ & $5.3 \times 5.3$ & 82.0 & $31.2 \mathrm{HDR}$ \\
\hline & & & $042948.8+262513$ & $042938.9+263827$ & $5.3 \times 5.3$ & 82.0 & $31.2 \mathrm{HDR}$ \\
\hline & & & $042920.9+263339$ & $042920.9+263339$ & $5.3 \times 5.3$ & 82.0 & $31.2 \mathrm{HDR}$ \\
\hline & & & $042925.5+262700$ & $042915.7+264020$ & $5.3 \times 5.3$ & 82.0 & $31.2 \mathrm{HDR}$ \\
\hline & & & $042905.1+264910$ & $042905.1+264910$ & $5.3 \times 5.3$ & 82.0 & $31.2 \mathrm{HDR}$ \\
\hline & & & $042910.7+264225$ & $042900.0+265552$ & $5.3 \times 5.3$ & 82.0 & $31.2 \mathrm{HDR}$ \\
\hline & & & $0431 \quad 14.6+27 \quad 1021$ & $043114.6+271021$ & $5.3 \times 5.3$ & 82.0 & $31.2 \mathrm{HDR}$ \\
\hline & & & $043119.7+270337$ & $043109.5+271705$ & $5.3 \times 5.3$ & 82.0 & $31.2 \mathrm{HDR}$ \\
\hline & & & $042703.1+254215$ & $042703.1+254215$ & $5.3 \times 5.3$ & 82.0 & $31.2 \mathrm{HDR}$ \\
\hline & & & $042707.9+253543$ & $042657.8+254858$ & $5.3 \times 5.3$ & 82.0 & $31.2 \mathrm{HDR}$ \\
\hline & & & $042659.3+260624$ & $042659.3+260624$ & $5.3 \times 5.3$ & 82.0 & $31.2 \mathrm{HDR}$ \\
\hline & & & $042704.2+255957$ & $042653.9+261307$ & $5.3 \times 5.3$ & 82.0 & $31.2 \mathrm{HDR}$ \\
\hline & & & $042727.7+261202$ & $042727.7+261202$ & $5.3 \times 5.3$ & 82.0 & $31.2 \mathrm{HDR}$ \\
\hline & & & $042733.4+260526$ & $042722.4+261846$ & $5.3 \times 5.3$ & 82.0 & $31.2 \mathrm{HDR}$ \\
\hline & & & $042758.2+261910$ & $042758.2+261910$ & $5.3 \times 5.3$ & 82.0 & $31.2 \mathrm{HDR}$ \\
\hline & & & $042802.2+261241$ & $042753.0+262552$ & $5.3 \times 5.3$ & 82.0 & $31.2 \mathrm{HDR}$ \\
\hline & & & $042629.4+262404$ & $042629.4+262404$ & $5.3 \times 5.3$ & 82.0 & $31.2 \mathrm{HDR}$ \\
\hline & & & $042634.7+261736$ & $042624.0+263048$ & $5.3 \times 5.3$ & 82.0 & $31.2 \mathrm{HDR}$ \\
\hline & & & $042517.3+261745$ & $042517.3+261745$ & $5.3 \times 5.3$ & 82.0 & $31.2 \mathrm{HDR}$ \\
\hline & & & $042523.3+261112$ & $042511.9+262426$ & $5.3 \times 5.3$ & 82.0 & $31.2 \mathrm{HDR}$ \\
\hline & & & $042445.1+261012$ & $042445.1+261012$ & $5.3 \times 5.3$ & 82.0 & $31.2 \mathrm{HDR}$ \\
\hline & & & $042450.1+260334$ & $042439.5+261657$ & $5.3 \times 5.3$ & 82.0 & $31.2 \mathrm{HDR}$ \\
\hline & & & $044009.7+260515$ & $044009.7+260515$ & $5.3 \times 5.3$ & 82.5 & $31.2 \mathrm{HDR}$ \\
\hline & & & $044013.8+255845$ & $044005.2+261158$ & $5.3 \times 5.3$ & 82.5 & $31.2 \mathrm{HDR}$ \\
\hline & & & $043950.6+260225$ & $043950.6+260225$ & $5.3 \times 5.3$ & 82.5 & $31.2 \mathrm{HDR}$ \\
\hline & & & $043955.6+255540$ & $043946.0+260908$ & $5.3 \times 5.3$ & 82.5 & $31.2 \mathrm{HDR}$ \\
\hline & & & $043913.7+255317$ & $043913.7+255317$ & $5.3 \times 5.3$ & 82.5 & $31.2 \mathrm{HDR}$ \\
\hline & & & $043918.4+254638$ & $043909.1+260000$ & $5.3 \times 5.3$ & 82.5 & $31.2 \mathrm{HDR}$ \\
\hline & & & $043921.4+254453$ & $043921.4+254453$ & $5.3 \times 5.3$ & 82.5 & $31.2 \mathrm{HDR}$ \\
\hline & & & $043925.5+253821$ & $043916.9+255136$ & $5.3 \times 5.3$ & 82.5 & $31.2 \mathrm{HDR}$ \\
\hline & & & $043936.0+254136$ & $043936.0+254136$ & $5.3 \times 5.3$ & 82.5 & $31.2 \mathrm{HDR}$ \\
\hline & & & $043939.4+253506$ & $043931.5+254818$ & $5.3 \times 5.3$ & 82.5 & $31.2 \mathrm{HDR}$ \\
\hline & & & $043956.0+254454$ & $043956.0+254454$ & $5.3 \times 5.3$ & 82.5 & $31.2 \mathrm{HDR}$ \\
\hline & & & $044000.6+253828$ & $043951.5+255137$ & $5.3 \times 5.3$ & 82.5 & $31.2 \mathrm{HDR}$ \\
\hline & & & $043828.7+261000$ & $043828.7+261000$ & $5.3 \times 5.3$ & 82.5 & $31.2 \mathrm{HDR}$ \\
\hline & & & $043833.0+260320$ & $043824.1+261644$ & $5.3 \times 5.3$ & 82.5 & $31.2 \mathrm{HDR}$ \\
\hline & & & $043619.3+254251$ & $043619.3+254251$ & $5.3 \times 5.3$ & 82.5 & $31.2 \mathrm{HDR}$ \\
\hline & & & $043623.6+253619$ & $043614.6+254933$ & $5.3 \times 5.3$ & 82.5 & $31.2 \mathrm{HDR}$ \\
\hline & & & $043339.0+252030$ & $043339.0+252030$ & $5.3 \times 5.3$ & 82.5 & $31.2 \mathrm{HDR}$ \\
\hline & & & $043343.9+251358$ & $043334.2+252713$ & $5.3 \times 5.3$ & 82.5 & $31.2 \mathrm{HDR}$ \\
\hline & & & $043354.7+261325$ & $043354.7+261325$ & $5.3 \times 5.3$ & 82.5 & $31.2 \mathrm{HDR}$ \\
\hline & & & $043400.0+260655$ & $043349.8+262006$ & $5.3 \times 5.3$ & 82.5 & $31.2 \mathrm{HDR}$ \\
\hline & & & $043336.5+260943$ & $043336.5+260943$ & $5.3 \times 5.3$ & 82.5 & $31.2 \mathrm{HDR}$ \\
\hline & & & $043341.8+260309$ & $043331.4+261626$ & $5.3 \times 5.3$ & 82.5 & $31.2 \mathrm{HDR}$ \\
\hline & & & $043314.2+261420$ & $043314.2+261420$ & $5.3 \times 5.3$ & 82.5 & $31.2 \mathrm{HDR}$ \\
\hline & & & $043319.3+260740$ & $043309.2+262102$ & $5.3 \times 5.3$ & 82.5 & $31.2 \mathrm{HDR}$ \\
\hline & & & $043243.2+255230$ & $043243.2+255230$ & $5.3 \times 5.3$ & 82.5 & $31.2 \mathrm{HDR}$ \\
\hline & & & $043247.6+254549$ & $043238.2+255912$ & $5.3 \times 5.3$ & 82.5 & $31.2 \mathrm{HDR}$ \\
\hline & & & $043158.1+254326$ & $043158.1+254326$ & $5.3 \times 5.3$ & 82.5 & $31.2 \mathrm{HDR}$ \\
\hline & & & $043203.5+253650$ & $043153.1+255008$ & $5.3 \times 5.3$ & 82.5 & $31.2 \mathrm{HDR}$ \\
\hline \multirow[t]{4}{*}{3966208} & \multirow[t]{4}{*}{37} & \multirow[t]{4}{*}{2004 Mar 8} & $042052.8+174643$ & $042052.8+174643$ & $5.3 \times 5.3$ & 81.5 & $31.2 \mathrm{HDR}$ \\
\hline & & & $042058.1+173959$ & $042047.8+175326$ & $5.3 \times 5.3$ & 81.5 & $31.2 \mathrm{HDR}$ \\
\hline & & & $041822.3+165835$ & $041822.3+165835$ & $5.3 \times 5.3$ & 81.5 & $31.2 \mathrm{HDR}$ \\
\hline & & & $041826.1+165208$ & $041817.3+170517$ & $5.3 \times 5.3$ & 81.5 & $31.2 \mathrm{HDR}$ \\
\hline \multirow[t]{2}{*}{3967744} & 37 & 2004 Mar 9 & $043916.0+303210$ & $043916.0+303210$ & $5.3 \times 5.3$ & 83.0 & $31.2 \mathrm{HDR}$ \\
\hline & & & $043921.4+302526$ & $043911.0+303853$ & $5.3 \times 5.3$ & 83.0 & $31.2 \mathrm{HDR}$ \\
\hline 4913152 & 94 & 2004 Mar 9 & $043409.3+240627$ & $043404.5+241309$ & $10.3 \times 19.0$ & 172.3 & $52 \mathrm{HDR}$ \\
\hline 4913664 & 94 & 2004 Mar 9 & $043614.5+244047$ & $043609.8+244730$ & $10.3 \times 15.0$ & 172.4 & $52 \mathrm{HDR}$ \\
\hline 4914176 & 94 & 2004 Mar 9 & $044041.0+295028$ & $044036.1+295712$ & $6.3 \times 15.0$ & 172.5 & $52 \mathrm{HDR}$ \\
\hline 4914688 & 94 & 2004 Mar 9 & $044332.7+293643$ & $044328.0+294325$ & $6.3 \times 15.0$ & 173.0 & $52 \mathrm{HDR}$ \\
\hline 4913920 & 94 & 2004 Mar 11 & $043614.5+244047$ & $043609.8+244730$ & $10.3 \times 15.0$ & 172.4 & $52 \mathrm{HDR}$ \\
\hline 4914432 & 94 & 2004 Mar 11 & $044041.0+295028$ & $044036.1+295712$ & $6.3 \times 15.0$ & 172.5 & $52 \mathrm{HDR}$ \\
\hline 4914944 & 94 & 2004 Mar 11 & $044332.7+293643$ & $044328.0+294325$ & $6.3 \times 15.0$ & 173.0 & $52 \mathrm{HDR}$ \\
\hline 4913408 & 94 & 2004 Mar 12 & $043409.3+240627$ & $043409.3+240627$ & $10.3 \times 19.0$ & 172.3 & $52 \mathrm{HDR}$ \\
\hline 5075712 & 139 & 2004 Sep 7 & $042835.6+265546$ & $042840.7+264903$ & $5.3 \times 15.0$ & 172.0 & 20.8 \\
\hline 5076736 & 139 & 2004 Sep 7 & $042934.7+265855$ & $042934.7+265855$ & $5.3 \times 5.3$ & 82.0 & 20.8 \\
\hline & & & $042930.4+270534$ & $042939.9+265212$ & $5.3 \times 5.3$ & 82.0 & 20.8 \\
\hline 5086464 & 139 & 2004 Sep 7 & $044236.0+294712$ & $044240.5+294028$ & $5.3 \times 19.7$ & 173.3 & 20.8 \\
\hline 5657856 & 173 & 2004 Sep 7 & $040922.3+171638$ & $040915.6+171540$ & $5.6 \times 5.6$ & 80.5 & $20.8 \mathrm{HDR}$ \\
\hline & & & $040910.4+172222$ & $040927.5+170957$ & & 80.5 & $20.8 \mathrm{HDR}$ \\
\hline 5658368 & 173 & 2004 Sep 7 & $041856.7+172345$ & $041850.1+172248$ & $5.6 \times 5.6$ & 81.5 & $20.8 \mathrm{HDR}$ \\
\hline & & & $041845.0+172930$ & $041901.8+171703$ & $5.6 \times 5.6$ & 81.5 & $20.8 \mathrm{HDR}$ \\
\hline 5658624 & 173 & 2004 Sep 7 & $042029.9+312355$ & $042022.7+312256$ & $5.6 \times 5.6$ & 81.0 & $20.8 \mathrm{HDR}$ \\
\hline & & & $042016.7+312937$ & $042035.9+311713$ & $5.6 \times 5.6$ & 81.0 & $20.8 \mathrm{HDR}$ \\
\hline 5658880 & 173 & 2004 Sep 7 & $042454.1+264342$ & $042447.1+264245$ & $5.6 \times 5.6$ & 82.0 & $20.8 \mathrm{HDR}$ \\
\hline
\end{tabular}


TABLE 1 - Continued

\begin{tabular}{|c|c|c|c|c|c|c|c|}
\hline AOR & PID & $\begin{array}{l}\text { Date } \\
\text { (UT) }\end{array}$ & $\begin{array}{c}\text { 3.6/5.8 Center } \\
(\mathrm{J} 2000)\end{array}$ & $\begin{array}{c}\text { 4.5/8.0 Center } \\
(\mathrm{J} 2000)\end{array}$ & $\begin{array}{l}\text { Dimensions } \\
\text { (arcmin) }\end{array}$ & $\begin{array}{c}\text { Angle }^{\mathrm{a}} \\
(\mathrm{deg})\end{array}$ & $\begin{array}{c}\text { Exp } \operatorname{Time}^{\mathrm{b}} \\
(\mathrm{s})\end{array}$ \\
\hline \multirow{7}{*}{5659136} & \multirow{7}{*}{173} & \multirow{6}{*}{2004 Sep 7} & $042441.6+264927$ & $042459.5+263702$ & $5.6 \times 5.6$ & 82.0 & $20.8 \mathrm{HDR}$ \\
\hline & & & $043440.0+250120$ & $043439.4+250031$ & $5.6 \times 5.6$ & 82.0 & $20.8 \mathrm{HDR}$ \\
\hline & & & $043434.8+250715$ & $043444.8+245438$ & $5.6 \times 5.6$ & 82.0 & $20.8 \mathrm{HDR}$ \\
\hline & & & $043601.9+235233$ & $043555.3+235138$ & $5.6 \times 5.6$ & 82.0 & $20.8 \mathrm{HDR}$ \\
\hline & & & $043550.8+235823$ & $043606.8+234547$ & $5.6 \times 5.6$ & 82.0 & $20.8 \mathrm{HDR}$ \\
\hline & & & $042926.6+243951$ & $042928.9+244016$ & $5.6 \times 5.6$ & 82.0 & $20.8 \mathrm{HDR}$ \\
\hline & & & $042924.0+244658$ & $042931.8+243309$ & $5.6 \times 5.6$ & 82.0 & $20.8 \mathrm{HDR}$ \\
\hline \multirow[t]{4}{*}{5659392} & \multirow[t]{4}{*}{173} & \multirow[t]{4}{*}{2004 Sep 7} & $043813.7+202259$ & $043813.5+202210$ & $5.6 \times 5.6$ & 83.0 & $20.8 \mathrm{HDR}$ \\
\hline & & & $043808.9+202855$ & $043818.0+201628$ & $5.6 \times 5.6$ & 83.0 & $20.8 \mathrm{HDR}$ \\
\hline & & & $043732.2+185154$ & $043725.4+185100$ & $5.6 \times 5.6$ & 83.0 & $20.8 \mathrm{HDR}$ \\
\hline & & & $043720.8+185742$ & $043736.3+184511$ & $5.6 \times 5.6$ & 83.0 & $20.8 \mathrm{HDR}$ \\
\hline \multirow{4}{*}{5659648} & 173 & 2004 Sep 7 & $044552.0+155604$ & $044551.6+155521$ & $5.6 \times 5.6$ & 83.5 & $20.8 \mathrm{HDR}$ \\
\hline & & & $044547.5+160207$ & $044555.9+154929$ & $5.6 \times 5.6$ & 83.5 & $20.8 \mathrm{HDR}$ \\
\hline & & & $043843.9+154641$ & $043837.5+154546$ & $5.6 \times 5.6$ & 83.5 & $20.8 \mathrm{HDR}$ \\
\hline & & & $043833.2+155230$ & $043848.2+153959$ & $5.6 \times 5.6$ & 83.5 & $20.8 \mathrm{HDR}$ \\
\hline 5660416 & 173 & 2004 Sep 7 & $045235.9+173050$ & $045229.3+173000$ & $5.6 \times 5.6$ & 84.0 & $20.8 \mathrm{HDR}$ \\
\hline & & & $045225.4+173645$ & $045239.9+172405$ & $5.6 \times 5.6$ & 84.0 & $20.8 \mathrm{HDR}$ \\
\hline & & & $045250.9+162231$ & $045250.2+162142$ & $5.6 \times 5.6$ & 84.0 & $20.8 \mathrm{HDR}$ \\
\hline & & & $045246.4+162821$ & $045254.7+161549$ & $5.6 \times 5.6$ & 84.0 & $20.8 \mathrm{HDR}$ \\
\hline 5660928 & 173 & 2004 Sep 7 & $045840.7+204652$ & $045840.0+204615$ & $5.6 \times 5.6$ & 84.5 & $20.8 \mathrm{HDR}$ \\
\hline & & & $045836.4+205301$ & $045844.0+204016$ & $5.6 \times 5.6$ & 84.5 & $20.8 \mathrm{HDR}$ \\
\hline & & & $045735.7+201455$ & $045729.0+201403$ & $5.6 \times 5.6$ & 84.5 & $20.8 \mathrm{HDR}$ \\
\hline & & & $045725.4+202048$ & $045739.4+200809$ & $5.6 \times 5.6$ & 84.5 & $20.8 \mathrm{HDR}$ \\
\hline 5661184 & 173 & 2004 Sep 7 & $050312.1+252344$ & $050304.9+252255$ & $5.6 \times 5.6$ & 85.5 & $20.8 \mathrm{HDR}$ \\
\hline & & & $050301.7+252940$ & $050315.2+251700$ & $5.6 \times 5.6$ & 85.5 & $20.8 \mathrm{HDR}$ \\
\hline 5672960 & 173 & 2004 Sep 7 & $041251.2+193727$ & $041251.1+193631$ & $5.6 \times 5.6$ & 81.0 & $20.8 \mathrm{HDR}$ \\
\hline & & & $041245.7+194303$ & $041256.7+193048$ & $5.6 \times 5.6$ & 81.0 & $20.8 \mathrm{HDR}$ \\
\hline & & & $040524.7+200953$ & $040518.2+200858$ & $5.6 \times 5.6$ & 81.0 & $20.8 \mathrm{HDR}$ \\
\hline & & & $040512.4+201537$ & $040530.4+200317$ & $5.6 \times 5.6$ & 81.0 & $20.8 \mathrm{HDR}$ \\
\hline 5710336 & 173 & 2004 Sep 7 & $043258.3+173601$ & $043251.7+173508$ & $5.6 \times 5.6$ & 82.5 & $20.8 \mathrm{HDR}$ \\
\hline & & & $043247.1+174150$ & $043303.0+172919$ & $5.6 \times 5.6$ & 82.5 & $20.8 \mathrm{HDR}$ \\
\hline 5073920 & 139 & 2004 Sep 8 & $042156.4+152949$ & $042156.4+152949$ & $5.3 \times 5.3$ & 82.0 & $20.8 \mathrm{HDR}$ \\
\hline & & & $042152.4+153627$ & $042201.1+152306$ & $5.3 \times 5.3$ & 82.0 & $20.8 \mathrm{HDR}$ \\
\hline 5658112 & 173 & 2004 Sep 8 & $040956.4+244652$ & $040949.7+244555$ & $5.6 \times 5.6$ & 80.0 & $20.8 \mathrm{HDR}$ \\
\hline & & & $040943.6+245234$ & $041002.6+244014$ & $5.6 \times 5.6$ & 80.0 & $20.8 \mathrm{HDR}$ \\
\hline 5660160 & 173 & 2004 Sep 8 & $044113.9+255635$ & $044106.8+255540$ & $5.6 \times 5.6$ & 83.5 & $20.8 \mathrm{HDR}$ \\
\hline & & & $044102.5+260227$ & $044118.4+254950$ & $5.6 \times 5.6$ & 83.5 & $20.8 \mathrm{HDR}$ \\
\hline 5076224 & 139 & 2004 Sep 9 & $042835.6+265546$ & $042840.7+264903$ & $5.3 \times 15.0$ & 172.0 & 20.8 \\
\hline 5077248 & 139 & 2004 Sep 9 & $042934.7+265855$ & $042934.7+265855$ & $5.3 \times 5.3$ & 82.0 & 20.8 \\
\hline & & & $042930.4+270534$ & $042939.9+265212$ & $5.3 \times 5.3$ & 82.0 & 20.8 \\
\hline 5074432 & 139 & 2004 Sep 10 & $042156.4+152949$ & $042156.4+152949$ & $5.3 \times 5.3$ & 82.0 & $20.8 \mathrm{HDR}$ \\
\hline & & & $042152.4+153627$ & $042201.1+152306$ & $5.3 \times 5.3$ & 82.0 & $20.8 \mathrm{HDR}$ \\
\hline 5086976 & 139 & 2004 Sep 10 & $044236.0+294712$ & $044240.5+294028$ & $5.3 \times 19.7$ & 173.3 & 20.8 \\
\hline 5074688 & 139 & 2004 Oct 5 & $042336.4+264007$ & $042336.4+264007$ & $5.3 \times 5.3$ & 79.5 & 20.8 \\
\hline & & & $042330.6+264646$ & $042342.9+263329$ & $5.3 \times 5.3$ & 79.5 & 20.8 \\
\hline 5077760 & 139 & 2004 Oct 5 & $043006.4+242555$ & $043006.4+242555$ & $5.3 \times 5.3$ & 81.5 & 20.8 \\
\hline & & & $043001.7+243234$ & $043011.8+241913$ & $5.3 \times 5.3$ & 81.5 & 20.8 \\
\hline 5078784 & 139 & 2004 Oct 5 & $043153.3+243611$ & $043158.4+242928$ & $10.0 \times 15.0$ & 171.5 & 20.8 \\
\hline 5079808 & 139 & 2004 Oct 5 & $043236.1+245205$ & $043236.1+245205$ & $5.3 \times 5.3$ & 81.5 & 20.8 \\
\hline & & & $043231.5+245845$ & $043241.5+244524$ & $5.3 \times 5.3$ & 81.5 & 20.8 \\
\hline 5080832 & 139 & 2004 Oct 5 & $043245.6+242738$ & $043250.9+242055$ & $10.0 \times 15.0$ & 171.5 & 20.8 \\
\hline 5081856 & 139 & 2004 Oct 5 & $043552.4+240933$ & $043553.2+240930$ & $5.3 \times 5.3$ & 82.0 & 20.8 \\
\hline & & & $043548.2+241612$ & $043557.3+240251$ & $5.3 \times 5.3$ & 82.0 & 20.8 \\
\hline 3653120 & 6 & 2004 Oct 7 & $043138.0+181420$ & $043141.3+180735$ & $28.5 \times 33.2$ & 175.0 & $20.8 \mathrm{HDR}$ \\
\hline 5075200 & 139 & 2004 Oct 7 & $042336.4+264007$ & $042336.4+264007$ & $5.3 \times 5.3$ & 79.5 & 20.8 \\
\hline & & & $042330.6+264646$ & $042342.9+263329$ & $5.3 \times 5.3$ & 79.5 & 20.8 \\
\hline 5078272 & 139 & 2004 Oct 7 & $043006.4+242555$ & $043006.4+242555$ & $5.3 \times 5.3$ & 81.5 & 20.8 \\
\hline & & & $043001.7+243234$ & $043011.8+241913$ & $5.3 \times 5.3$ & 81.5 & 20.8 \\
\hline 5079296 & 139 & 2004 Oct 7 & $043153.3+243611$ & $043158.4+242928$ & $10.0 \times 15.0$ & 171.5 & 20.8 \\
\hline 5080320 & 139 & 2004 Oct 7 & $043236.1+245205$ & $043236.1+245205$ & $5.3 \times 5.3$ & 81.5 & 20.8 \\
\hline & & & $043231.5+245845$ & $043241.5+244524$ & $5.3 \times 5.3$ & 81.5 & 20.8 \\
\hline 5081344 & 139 & 2004 Oct 7 & $043245.6+242738$ & $043250.9+242055$ & $10.0 \times 15.0$ & 171.5 & 20.8 \\
\hline 5082368 & 139 & 2004 Oct 7 & $043552.4+240933$ & $043553.2+240930$ & $5.3 \times 5.3$ & 82.0 & 20.8 \\
\hline & & & $043548.2+241612$ & $043557.3+240251$ & $5.3 \times 5.3$ & 82.0 & 20.8 \\
\hline 5082880 & 139 & 2004 Oct 8 & $044054.8+261324$ & $044059.8+260644$ & $10.0 \times 19.5$ & 171.5 & 20.8 \\
\hline 5083392 & 139 & 2004 Oct 8 & $044054.8+261324$ & $044059.8+260644$ & $10.0 \times 19.5$ & 171.5 & 20.8 \\
\hline 5084416 & 139 & 2004 Oct 8 & $044129.8+260412$ & $044134.8+255728$ & $10.0 \times 24.0$ & 171.6 & $20.8 \mathrm{HDR}$ \\
\hline 5084928 & 139 & 2004 Oct 8 & $044129.8+260412$ & $044134.8+255728$ & $10.0 \times 24.0$ & 171.6 & $20.8 \mathrm{HDR}$ \\
\hline 5085440 & 139 & 2004 Oct 8 & $044141.0+254547$ & $044146.1+253905$ & $10.0 \times 19.5$ & 171.8 & 20.8 \\
\hline 5085952 & 139 & 2004 Oct 8 & $044141.0+254547$ & $044146.1+253905$ & $10.0 \times 19.5$ & 171.8 & 20.8 \\
\hline 6634752 & 139 & 2004 Oct 8 & $044057.1+255727$ & $044102.1+255046$ & $10.0 \times 19.5$ & 171.6 & 20.8 \\
\hline 6635008 & 139 & 2004 Oct 8 & $044057.1+255727$ & $044102.1+255046$ & $10.0 \times 19.5$ & 171.6 & 20.8 \\
\hline 4912640 & 94 & 2005 Feb 18 & $041055.7+250543$ & $041050.1+251224$ & $10.4 \times 23.0$ & 170.9 & $52 \mathrm{HDR}$ \\
\hline 3653888 & 6 & 2005 Feb 19 & $041428.7+281219$ & $041423.8+281902$ & $28.6 \times 33.2$ & 172.3 & $20.8 \mathrm{HDR}$ \\
\hline 3965184 & 37 & 2005 Feb 19 & $043127.5+170624$ & $043127.5+170624$ & $5.3 \times 5.3$ & 80.0 & $31.2 \mathrm{HDR}$ \\
\hline
\end{tabular}


TABLE 1 - Continued

\begin{tabular}{|c|c|c|c|c|c|c|c|}
\hline AOR & PID & $\begin{array}{l}\text { Date } \\
\text { (UT) }\end{array}$ & $\begin{array}{c}\text { 3.6/5.8 Center } \\
(\mathrm{J} 2000)\end{array}$ & $\begin{array}{c}\text { 4.5/8.0 Center } \\
(\mathrm{J} 2000)\end{array}$ & $\begin{array}{l}\text { Dimensions } \\
\text { (arcmin) }\end{array}$ & $\begin{array}{c}\text { Angle }^{\mathrm{a}} \\
(\mathrm{deg})\end{array}$ & $\operatorname{Exp}_{(\mathrm{s})} \operatorname{Time}^{\mathrm{b}}$ \\
\hline & & & $043132.3+165943$ & $043121.9+171304$ & $5.3 \times 5.3$ & 80.0 & $31.2 \mathrm{HDR}$ \\
\hline & & & $043230.4+173138$ & $043230.4+173138$ & $5.3 \times 5.3$ & 80.0 & $31.2 \mathrm{HDR}$ \\
\hline & & & $043236.0+172453$ & $043224.8+173818$ & $5.3 \times 5.3$ & 80.0 & $31.2 \mathrm{HDR}$ \\
\hline & & & $043339.5+175148$ & $043339.5+175148$ & $5.3 \times 5.3$ & 80.0 & $31.2 \mathrm{HDR}$ \\
\hline & & & $043345.1+174514$ & $043334.0+175829$ & $5.3 \times 5.3$ & 80.0 & $31.2 \mathrm{HDR}$ \\
\hline & & & $043524.3+175138$ & $043524.3+175138$ & $5.3 \times 5.3$ & 80.0 & $31.2 \mathrm{HDR}$ \\
\hline & & & $043530.0+174505$ & $043518.9+175819$ & $5.3 \times 5.3$ & 80.0 & $31.2 \mathrm{HDR}$ \\
\hline & & & $043209.4+175719$ & $043209.4+175719$ & $5.3 \times 5.3$ & 80.0 & $31.2 \mathrm{HDR}$ \\
\hline & & & $043214.4+175044$ & $043203.9+180359$ & $5.3 \times 5.3$ & 80.0 & $31.2 \mathrm{HDR}$ \\
\hline & & & $043244.0+180303$ & $043244.0+180303$ & $5.3 \times 5.3$ & 80.0 & $31.2 \mathrm{HDR}$ \\
\hline & & & $043249.0+175617$ & $043238.5+180944$ & $5.3 \times 5.3$ & 80.0 & $31.2 \mathrm{HDR}$ \\
\hline & & & $043348.6+181009$ & $043348.6+181009$ & $5.3 \times 5.3$ & 80.0 & $31.2 \mathrm{HDR}$ \\
\hline & & & $043354.9+180323$ & $043343.1+181651$ & $5.3 \times 5.3$ & 80.0 & $31.2 \mathrm{HDR}$ \\
\hline & & & $043417.9+183002$ & $043417.9+183002$ & $5.3 \times 5.3$ & 80.0 & $31.2 \mathrm{HDR}$ \\
\hline & & & $043423.6+182322$ & $043412.6+183644$ & $5.3 \times 5.3$ & 80.0 & $31.2 \mathrm{HDR}$ \\
\hline & & & $043355.8+183844$ & $043355.8+183844$ & $5.3 \times 5.3$ & 80.0 & $31.2 \mathrm{HDR}$ \\
\hline & & & $043401.1+183154$ & $043350.6+184525$ & $5.3 \times 5.3$ & 80.0 & $31.2 \mathrm{HDR}$ \\
\hline & & & $043222.2+182732$ & $043222.2+182732$ & $5.3 \times 5.3$ & 80.0 & $31.2 \mathrm{HDR}$ \\
\hline & & & $043227.9+182052$ & $043216.8+183414$ & $5.3 \times 5.3$ & 80.0 & $31.2 \mathrm{HDR}$ \\
\hline & & & $043004.6+181345$ & $043004.6+181345$ & $5.3 \times 5.3$ & 80.0 & $31.2 \mathrm{HDR}$ \\
\hline & & & $043009.5+180715$ & $042959.1+182025$ & $5.3 \times 5.3$ & 80.0 & $31.2 \mathrm{HDR}$ \\
\hline & & & $042710.7+175040$ & $042710.7+175040$ & $5.3 \times 5.3$ & 80.0 & $31.2 \mathrm{HDR}$ \\
\hline & & & $042716.1+174358$ & $042705.0+175721$ & $5.3 \times 5.3$ & 80.0 & $31.2 \mathrm{HDR}$ \\
\hline \multirow[t]{16}{*}{3962880} & 37 & 2005 Feb 20 & $041901.4+280244$ & $041901.4+280244$ & $5.3 \times 5.3$ & 83.0 & $31.2 \mathrm{HDR}$ \\
\hline & & & $041905.7+275607$ & $041856.5+280928$ & $5.3 \times 5.3$ & 83.0 & $31.2 \mathrm{HDR}$ \\
\hline & & & $041738.9+283259$ & $041738.9+283259$ & $5.3 \times 5.3$ & 83.0 & $31.2 \mathrm{HDR}$ \\
\hline & & & $041744.2+282613$ & $041734.1+283941$ & $5.3 \times 5.3$ & 83.0 & $31.2 \mathrm{HDR}$ \\
\hline & & & $041915.7+290627$ & $041915.7+290627$ & $5.3 \times 5.3$ & 83.0 & $31.2 \mathrm{HDR}$ \\
\hline & & & $041921.2+285938$ & $041911.0+291310$ & $5.3 \times 5.3$ & 83.0 & $31.2 \mathrm{HDR}$ \\
\hline & & & $041514.6+280008$ & $041514.6+280008$ & $5.3 \times 5.3$ & 83.0 & $31.2 \mathrm{HDR}$ \\
\hline & & & $041519.9+275323$ & $041509.5+280651$ & $5.3 \times 5.3$ & 83.0 & $31.2 \mathrm{HDR}$ \\
\hline & & & $041448.4+275240$ & $041448.4+275240$ & $5.3 \times 5.3$ & 83.0 & $31.2 \mathrm{HDR}$ \\
\hline & & & $041453.2+274548$ & $041443.3+275924$ & $5.3 \times 5.3$ & 83.0 & $31.2 \mathrm{HDR}$ \\
\hline & & & $041327.3+281623$ & $041327.3+281623$ & $5.3 \times 5.3$ & 83.0 & $31.2 \mathrm{HDR}$ \\
\hline & & & $041332.4+280937$ & $041322.3+282306$ & $5.3 \times 5.3$ & 83.0 & $31.2 \mathrm{HDR}$ \\
\hline & & & $041314.3+281909$ & $041314.3+281909$ & $5.3 \times 5.3$ & 83.0 & $31.2 \mathrm{HDR}$ \\
\hline & & & $041319.5+281233$ & $041309.2+282552$ & $5.3 \times 5.3$ & 83.0 & $31.2 \mathrm{HDR}$ \\
\hline & & & $041358.1+291816$ & $041358.1+291816$ & $5.3 \times 5.3$ & 83.0 & $31.2 \mathrm{HDR}$ \\
\hline & & & $041402.4+291143$ & $041353.1+292458$ & $5.3 \times 5.3$ & 83.0 & $31.2 \mathrm{HDR}$ \\
\hline \multirow[t]{22}{*}{3963136} & 37 & 2005 Feb 20 & $042444.8+270141$ & $042444.8+270141$ & $5.3 \times 5.3$ & 83.5 & $31.2 \mathrm{HDR}$ \\
\hline & & & $042449.8+265503$ & $042440.2+270825$ & $5.3 \times 5.3$ & 83.5 & $31.2 \mathrm{HDR}$ \\
\hline & & & $042457.0+271154$ & $042457.0+271154$ & $5.3 \times 5.3$ & 83.5 & $31.2 \mathrm{HDR}$ \\
\hline & & & $042501.9+270510$ & $042452.4+271838$ & $5.3 \times 5.3$ & 83.5 & $31.2 \mathrm{HDR}$ \\
\hline & & & $042202.6+265736$ & $042202.6+265736$ & $5.3 \times 5.3$ & 83.5 & $31.2 \mathrm{HDR}$ \\
\hline & & & $042207.1+265044$ & $042157.8+270420$ & $5.3 \times 5.3$ & 83.5 & $31.2 \mathrm{HDR}$ \\
\hline & & & $042110.2+270139$ & $042110.2+270139$ & $5.3 \times 5.3$ & 83.5 & $31.2 \mathrm{HDR}$ \\
\hline & & & $042115.2+265502$ & $042105.4+270822$ & $5.3 \times 5.3$ & 83.5 & $31.2 \mathrm{HDR}$ \\
\hline & & & $041958.2+270958$ & $041958.2+270958$ & $5.3 \times 5.3$ & 83.5 & $31.2 \mathrm{HDR}$ \\
\hline & & & $042004.5+270309$ & $041953.4+271639$ & $5.3 \times 5.3$ & 83.5 & $31.2 \mathrm{HDR}$ \\
\hline & & & $041943.6+271330$ & $041943.6+271330$ & $5.3 \times 5.3$ & 83.5 & $31.2 \mathrm{HDR}$ \\
\hline & & & $041947.8+270659$ & $041938.8+272014$ & $5.3 \times 5.3$ & 83.5 & $31.2 \mathrm{HDR}$ \\
\hline & & & $041941.5+274948$ & $041941.5+274948$ & $5.3 \times 5.3$ & 83.5 & $31.2 \mathrm{HDR}$ \\
\hline & & & $041945.6+274304$ & $041936.8+275629$ & $5.3 \times 5.3$ & 83.5 & $31.2 \mathrm{HDR}$ \\
\hline & & & $042155.7+275503$ & $042155.7+275503$ & $5.3 \times 5.3$ & 83.5 & $31.2 \mathrm{HDR}$ \\
\hline & & & $042200.4+274817$ & $042151.0+280148$ & $5.3 \times 5.3$ & 83.5 & $31.2 \mathrm{HDR}$ \\
\hline & & & $042311.7+280559$ & $042311.7+280559$ & $5.3 \times 5.3$ & 83.5 & $31.2 \mathrm{HDR}$ \\
\hline & & & $042316.6+275914$ & $042307.1+281244$ & $5.3 \times 5.3$ & 83.5 & $31.2 \mathrm{HDR}$ \\
\hline & & & $042159.1+281813$ & $042159.1+281813$ & $5.3 \times 5.3$ & 83.5 & $31.2 \mathrm{HDR}$ \\
\hline & & & $042203.4+281127$ & $042154.4+282458$ & $5.3 \times 5.3$ & 83.5 & $31.2 \mathrm{HDR}$ \\
\hline & & & $042200.4+282604$ & $042200.4+282604$ & $5.3 \times 5.3$ & 83.5 & $31.2 \mathrm{HDR}$ \\
\hline & & & $042204.6+281926$ & $042155.8+283249$ & $5.3 \times 5.3$ & 83.5 & $31.2 \mathrm{HDR}$ \\
\hline \multirow{14}{*}{3964160} & 37 & 2005 Feb 20 & $043917.9+222058$ & $043917.9+222058$ & $5.3 \times 5.3$ & 83.0 & $31.2 \mathrm{HDR}$ \\
\hline & & & $043922.0+221423$ & $043913.3+222742$ & $5.3 \times 5.3$ & 83.0 & $31.2 \mathrm{HDR}$ \\
\hline & & & $043917.8+224745$ & $043917.8+224745$ & $5.3 \times 5.3$ & 83.0 & $31.2 \mathrm{HDR}$ \\
\hline & & & $043921.9+224119$ & $043913.3+225428$ & $5.3 \times 5.3$ & 83.0 & $31.2 \mathrm{HDR}$ \\
\hline & & & $043520.3+223210$ & $043520.3+223210$ & $5.3 \times 5.3$ & 83.0 & $31.2 \mathrm{HDR}$ \\
\hline & & & $043524.7+222531$ & $043515.6+223853$ & $5.3 \times 5.3$ & 83.0 & $31.2 \mathrm{HDR}$ \\
\hline & & & $043542.2+223409$ & $043542.2+223409$ & $5.3 \times 5.3$ & 83.0 & $31.2 \mathrm{HDR}$ \\
\hline & & & $043546.1+222728$ & $043537.4+224054$ & $5.3 \times 5.3$ & 83.0 & $31.2 \mathrm{HDR}$ \\
\hline & & & $043639.1+225810$ & $043639.1+225810$ & $5.3 \times 5.3$ & 83.0 & $31.2 \mathrm{HDR}$ \\
\hline & & & $043643.1+225130$ & $043634.6+230454$ & $5.3 \times 5.3$ & 83.0 & $31.2 \mathrm{HDR}$ \\
\hline & & & $043610.4+225956$ & $043610.4+225956$ & $5.3 \times 5.3$ & 83.0 & $31.2 \mathrm{HDR}$ \\
\hline & & & $043614.9+225310$ & $043605.8+230637$ & $5.3 \times 5.3$ & 83.0 & $31.2 \mathrm{HDR}$ \\
\hline & & & $043552.7+225421$ & $043552.7+225421$ & $5.3 \times 5.3$ & 83.0 & $31.2 \mathrm{HDR}$ \\
\hline & & & $043557.5+224737$ & $043548.1+230104$ & $5.3 \times 5.3$ & 83.0 & $31.2 \mathrm{HDR}$ \\
\hline
\end{tabular}


TABLE 1 - Continued

\begin{tabular}{|c|c|c|c|c|c|c|c|}
\hline AOR & PID & $\begin{array}{l}\text { Date } \\
\text { (UT) }\end{array}$ & $\begin{array}{c}\text { 3.6/5.8 Center } \\
(\mathrm{J} 2000)\end{array}$ & $\begin{array}{l}\text { 4.5/8.0 Center } \\
(\mathrm{J} 2000)\end{array}$ & $\begin{array}{l}\text { Dimensions } \\
\text { (arcmin) }\end{array}$ & $\begin{array}{c}\text { Angle }^{\mathrm{a}} \\
(\mathrm{deg})\end{array}$ & $\operatorname{Exp}_{(\mathrm{s})} \operatorname{Time}^{\mathrm{b}}$ \\
\hline \multirow{46}{*}{3964416} & \multirow{46}{*}{37} & \multirow{46}{*}{2005 Feb 20} & $043551.1+224909$ & $043551.1+224909$ & $5.3 \times 5.3$ & 83.0 & $31.2 \mathrm{HDR}$ \\
\hline & & & $043556.2+224231$ & $043546.6+225552$ & $5.3 \times 5.3$ & 83.0 & $31.2 \mathrm{HDR}$ \\
\hline & & & $043521.1+225423$ & $043521.1+225423$ & $5.3 \times 5.3$ & 83.0 & $31.2 \mathrm{HDR}$ \\
\hline & & & $043525.9+224744$ & $043516.4+230105$ & $5.3 \times 5.3$ & 83.0 & $31.2 \mathrm{HDR}$ \\
\hline & & & $043413.4+225113$ & $043413.4+225113$ & $5.3 \times 5.3$ & 83.0 & $31.2 \mathrm{HDR}$ \\
\hline & & & $043418.1+224427$ & $043408.6+225756$ & $5.3 \times 5.3$ & 83.0 & $31.2 \mathrm{HDR}$ \\
\hline & & & $043352.5+225036$ & $043352.5+225036$ & $5.3 \times 5.3$ & 83.0 & $31.2 \mathrm{HDR}$ \\
\hline & & & $043356.9+224343$ & $043347.6+225718$ & $5.3 \times 5.3$ & 83.0 & $31.2 \mathrm{HDR}$ \\
\hline & & & $043316.2+225323$ & $043316.2+225323$ & $5.3 \times 5.3$ & 83.0 & $31.2 \mathrm{HDR}$ \\
\hline & & & $043321.7+224631$ & $043311.6+230004$ & $5.3 \times 5.3$ & 83.0 & $31.2 \mathrm{HDR}$ \\
\hline & & & $043319.1+224636$ & $043319.1+224636$ & $5.3 \times 5.3$ & 83.0 & $31.2 \mathrm{HDR}$ \\
\hline & & & $043324.2+223946$ & $043314.5+225315$ & $5.3 \times 5.3$ & 83.0 & $31.2 \mathrm{HDR}$ \\
\hline & & & $043249.2+225301$ & $043249.2+225301$ & $5.3 \times 5.3$ & 83.0 & $31.2 \mathrm{HDR}$ \\
\hline & & & $043254.0+224613$ & $043245.7+225949$ & $5.3 \times 5.3$ & 83.0 & $31.2 \mathrm{HDR}$ \\
\hline & & & $043232.6+225723$ & $043232.6+225723$ & $5.3 \times 5.3$ & 83.0 & $31.2 \mathrm{HDR}$ \\
\hline & & & $043236.8+225051$ & $043227.9+230406$ & $5.3 \times 5.3$ & 83.0 & $31.2 \mathrm{HDR}$ \\
\hline & & & $043050.4+225957$ & $043050.4+225957$ & $5.3 \times 5.3$ & 82.5 & $31.2 \mathrm{HDR}$ \\
\hline & & & $043054.9+225331$ & $043045.4+230641$ & $5.3 \times 5.3$ & 82.5 & $31.2 \mathrm{HDR}$ \\
\hline & & & $043540.7+241105$ & $043540.7+241105$ & $5.3 \times 5.3$ & 82.5 & $31.2 \mathrm{HDR}$ \\
\hline & & & $043545.4+240428$ & $043536.2+241749$ & $5.3 \times 5.3$ & 82.5 & $31.2 \mathrm{HDR}$ \\
\hline & & & $043536.0+240812$ & $043536.0+240812$ & $5.3 \times 5.3$ & 82.5 & $31.2 \mathrm{HDR}$ \\
\hline & & & $043539.9+240144$ & $043531.5+241456$ & $5.3 \times 5.3$ & 82.5 & $31.2 \mathrm{HDR}$ \\
\hline & & & $043527.7+241457$ & $043527.7+241457$ & $5.3 \times 5.3$ & 82.5 & $31.2 \mathrm{HDR}$ \\
\hline & & & $043531.5+240818$ & $043523.2+242140$ & $5.3 \times 5.3$ & 82.5 & $31.2 \mathrm{HDR}$ \\
\hline & & & $043455.7+242900$ & $043455.7+242900$ & $5.3 \times 5.3$ & 82.5 & $31.2 \mathrm{HDR}$ \\
\hline & & & $043500.0+242211$ & $043451.2+243545$ & $5.3 \times 5.3$ & 82.5 & $31.2 \mathrm{HDR}$ \\
\hline & & & $043306.4+240930$ & $043306.4+240930$ & $5.3 \times 5.3$ & 82.5 & $31.2 \mathrm{HDR}$ \\
\hline & & & $043310.5+240252$ & $043301.8+241611$ & $5.3 \times 5.3$ & 82.5 & $31.2 \mathrm{HDR}$ \\
\hline & & & $043310.1+243343$ & $043310.1+243343$ & $5.3 \times 5.3$ & 82.5 & $31.2 \mathrm{HDR}$ \\
\hline & & & $043314.4+242657$ & $043305.6+244025$ & $5.3 \times 5.3$ & 82.5 & $31.2 \mathrm{HDR}$ \\
\hline & & & $043334.5+242103$ & $043334.5+242103$ & $5.3 \times 5.3$ & 82.5 & $31.2 \mathrm{HDR}$ \\
\hline & & & $043339.3+241419$ & $043330.1+242747$ & $5.3 \times 5.3$ & 82.5 & $31.2 \mathrm{HDR}$ \\
\hline & & & $043302.4+242106$ & $043302.4+242106$ & $5.3 \times 5.3$ & 82.5 & $31.2 \mathrm{HDR}$ \\
\hline & & & $043306.7+241413$ & $043257.9+242748$ & $5.3 \times 5.3$ & 82.5 & $31.2 \mathrm{HDR}$ \\
\hline & & & $043231.9+241958$ & $043231.9+241958$ & $5.3 \times 5.3$ & 82.5 & $31.2 \mathrm{HDR}$ \\
\hline & & & $043236.7+241315$ & $043227.5+242643$ & $5.3 \times 5.3$ & 82.5 & $31.2 \mathrm{HDR}$ \\
\hline & & & $043215.7+242858$ & $043215.7+242858$ & $5.3 \times 5.3$ & 82.5 & $31.2 \mathrm{HDR}$ \\
\hline & & & $043220.0+242218$ & $043211.0+243540$ & $5.3 \times 5.3$ & 82.5 & $31.2 \mathrm{HDR}$ \\
\hline & & & $043218.7+242226$ & $043218.7+242226$ & $5.3 \times 5.3$ & 82.5 & $31.2 \mathrm{HDR}$ \\
\hline & & & $043222.9+241544$ & $043214.2+242909$ & $5.3 \times 5.3$ & 82.5 & $31.2 \mathrm{HDR}$ \\
\hline & & & $043150.5+242419$ & $043150.5+242419$ & $5.3 \times 5.3$ & 82.5 & $31.2 \mathrm{HDR}$ \\
\hline & & & $043155.9+241731$ & $043145.9+243104$ & $5.3 \times 5.3$ & 82.5 & $31.2 \mathrm{HDR}$ \\
\hline & & & $043123.9+241049$ & $043123.9+241049$ & $5.3 \times 5.3$ & 82.5 & $31.2 \mathrm{HDR}$ \\
\hline & & & $043128.1+240411$ & $043119.2+241731$ & $5.3 \times 5.3$ & 82.5 & $31.2 \mathrm{HDR}$ \\
\hline & & & $043029.8+242643$ & $043029.8+242643$ & $5.3 \times 5.3$ & 82.5 & $31.2 \mathrm{HDR}$ \\
\hline & & & $043034.4+241955$ & $043025.1+243325$ & $5.3 \times 5.3$ & 82.5 & $31.2 \mathrm{HDR}$ \\
\hline \multirow{4}{*}{3965952} & \multirow[t]{4}{*}{37} & \multirow{4}{*}{2005 Feb 20} & $042200.1+193202$ & $042200.1+193202$ & $5.3 \times 5.3$ & 80.5 & $31.2 \mathrm{HDR}$ \\
\hline & & & $042205.0+192533$ & $042154.5+193842$ & $5.3 \times 5.3$ & 80.5 & $31.2 \mathrm{HDR}$ \\
\hline & & & $042143.0+193414$ & $042143.0+193414$ & $5.3 \times 5.3$ & 80.5 & $31.2 \mathrm{HDR}$ \\
\hline & & & $042149.5+192729$ & $042137.5+194054$ & $5.3 \times 5.3$ & 80.5 & $31.2 \mathrm{HDR}$ \\
\hline \multirow[t]{6}{*}{3966976} & 37 & 2005 Feb 20 & $040443.6+261852$ & $040443.6+261852$ & $5.3 \times 5.3$ & 80.5 & $31.2 \mathrm{HDR}$ \\
\hline & & & $040448.6+261223$ & $040437.8+262534$ & $5.3 \times 5.3$ & 80.5 & $31.2 \mathrm{HDR}$ \\
\hline & & & $040350.7+261056$ & $040350.7+261056$ & $5.3 \times 5.3$ & 80.5 & $31.2 \mathrm{HDR}$ \\
\hline & & & $040357.4+260410$ & $040344.9+261735$ & $5.3 \times 5.3$ & 80.5 & $31.2 \mathrm{HDR}$ \\
\hline & & & $040314.1+255258$ & $040314.1+255258$ & $5.3 \times 5.3$ & 80.5 & $31.2 \mathrm{HDR}$ \\
\hline & & & $040320.1+254623$ & $040308.2+255937$ & $5.3 \times 5.3$ & 80.5 & $31.2 \mathrm{HDR}$ \\
\hline 4912896 & 94 & 2005 Feb 20 & $041055.7+250543$ & $041050.1+251224$ & $10.4 \times 23.0$ & 170.9 & $52 \mathrm{HDR}$ \\
\hline 11233024 & 3584 & 2005 Feb 20 & $042807.7+241406$ & $042803.2+242049$ & $34.2 \times 184.2$ & 172.8 & 10.4 HDR \\
\hline 11233536 & 3584 & 2005 Feb 20 & $042534.4+240936$ & $042529.4+241619$ & $34.2 \times 184.0$ & 172.6 & $10.4 \mathrm{HDR}$ \\
\hline 11236864 & 3584 & 2005 Feb 20 & $042807.7+241406$ & $042803.2+242049$ & $34.2 \times 184.2$ & 172.8 & $10.4 \mathrm{HDR}$ \\
\hline 11237376 & 3584 & 2005 Feb 20 & $042534.4+240936$ & $042529.4+241619$ & $34.2 \times 184.0$ & 172.6 & $10.4 \mathrm{HDR}$ \\
\hline 11237632 & 3584 & 2005 Feb 20 & $041922.5+274724$ & $041917.9+275411$ & $63.3 \times 82.5$ & 172.4 & $10.4 \mathrm{HDR}$ \\
\hline 12663552 & 37 & 2005 Feb 20 & $045748.9+301517$ & $045748.9+301517$ & $5.3 \times 5.3$ & 88.0 & 31.2 \\
\hline & & & $045751.2+300828$ & $045746.8+302204$ & $5.3 \times 5.3$ & 88.0 & 31.2 \\
\hline & & & $045552.7+300651$ & $045552.7+300651$ & $5.3 \times 5.3$ & 88.0 & 31.2 \\
\hline & & & $045555.8+300000$ & $045550.3+301339$ & $5.3 \times 5.3$ & 88.0 & 31.2 \\
\hline & & & $045545.3+301935$ & $045545.3+301935$ & $5.3 \times 5.3$ & 88.0 & 31.2 \\
\hline & & & $045548.1+301247$ & $045543.3+302619$ & $5.3 \times 5.3$ & 88.0 & 31.2 \\
\hline & & & $045548.7+302801$ & $045548.7+302801$ & $5.3 \times 5.3$ & 88.0 & 31.2 \\
\hline & & & $045550.2+302126$ & $045546.6+303446$ & $5.3 \times 5.3$ & 88.0 & 31.2 \\
\hline & & & $045523.5+302732$ & $045523.5+302732$ & $5.3 \times 5.3$ & 88.0 & 31.2 \\
\hline & & & $045525.4+302042$ & $045521.4+303421$ & $5.3 \times 5.3$ & 88.0 & 31.2 \\
\hline & & & $045540.7+303904$ & $045540.7+303904$ & $5.3 \times 5.3$ & 88.0 & 31.2 \\
\hline & & & $045542.9+303225$ & $045538.6+304550$ & $5.3 \times 5.3$ & 88.0 & 31.2 \\
\hline & & & $045556.8+304941$ & $045556.8+304941$ & $5.3 \times 5.3$ & 88.0 & 31.2 \\
\hline
\end{tabular}


TABLE 1 - Continued

\begin{tabular}{|c|c|c|c|c|c|c|c|}
\hline AOR & PID & $\begin{array}{l}\text { Date } \\
\text { (UT) }\end{array}$ & $\begin{array}{c}\text { 3.6/5.8 Center } \\
(\mathrm{J} 2000)\end{array}$ & $\begin{array}{c}\text { 4.5/8.0 Center } \\
(\mathrm{J} 2000)\end{array}$ & $\begin{array}{l}\text { Dimensions } \\
\text { (arcmin) }\end{array}$ & $\begin{array}{c}\text { Angle }^{\mathrm{a}} \\
(\mathrm{deg})\end{array}$ & $\operatorname{Exp}_{(\mathrm{s})} \operatorname{Time}^{\mathrm{b}}$ \\
\hline & & & $045558.7+304245$ & $045554.8+305629$ & $5.3 \times 5.3$ & 88.0 & 31.2 \\
\hline 12914944 & 3584 & 2005 Feb 20 & $043309.7+285614$ & $043305.8+290257$ & $53.7 \times 121.3$ & 174.4 & 10.4 HDR \\
\hline 12915712 & 3584 & 2005 Feb 20 & $043309.7+285614$ & $043305.8+290257$ & $53.7 \times 121.3$ & 174.4 & $10.4 \mathrm{HDR}$ \\
\hline 11231488 & 3584 & 2005 Feb 21 & $043621.8+240529$ & $043618.2+241208$ & $39.1 \times 150.4$ & 173.4 & $10.4 \mathrm{HDR}$ \\
\hline 11232000 & 3584 & 2005 Feb 21 & $043315.1+242230$ & $043310.8+242915$ & $39.0 \times 184.2$ & 173.0 & $10.4 \mathrm{HDR}$ \\
\hline 11233280 & 3584 & 2005 Feb 21 & $042349.0+270742$ & $042343.6+271424$ & $34.2 \times 184.0$ & 172.1 & $10.4 \mathrm{HDR}$ \\
\hline 11233792 & 3584 & 2005 Feb 21 & $041922.5+274724$ & $041917.9+275411$ & $63.3 \times 82.5$ & 172.4 & 10.4 HDR \\
\hline 11235328 & 3584 & 2005 Feb 21 & $043621.8+240529$ & $043618.2+241208$ & $39.1 \times 150.4$ & 173.4 & 10.4 HDR \\
\hline 11235840 & 3584 & 2005 Feb 21 & $043315.1+242230$ & $043310.8+242915$ & $39.0 \times 184.2$ & 173.0 & 10.4 HDR \\
\hline 11237120 & 3584 & 2005 Feb 21 & $042349.0+270742$ & $042343.6+271424$ & $34.2 \times 184.0$ & 172.1 & $10.4 \mathrm{HDR}$ \\
\hline 3653376 & 6 & 2005 Feb 22 & $041840.7+281642$ & $041835.9+282324$ & $28.6 \times 33.2$ & 171.8 & $20.8 \mathrm{HDR}$ \\
\hline 3653632 & 6 & 2005 Feb 22 & $041636.8+281427$ & $041631.7+282109$ & $28.6 \times 33.2$ & 171.8 & $20.8 \mathrm{HDR}$ \\
\hline \multirow{2}{*}{5657600} & 173 & 2005 Feb 22 & $040525.7+215040$ & $040532.3+215139$ & $5.6 \times 5.6$ & 80.0 & $20.8 \mathrm{HDR}$ \\
\hline & & & $040538.2+214457$ & $040519.6+215721$ & $5.6 \times 5.6$ & 80.0 & $20.8 \mathrm{HDR}$ \\
\hline 11230720 & 3584 & 2005 Feb 22 & $043918.8+243141$ & $043914.7+243823$ & $48.7 \times 121.4$ & 173.3 & $10.4 \mathrm{HDR}$ \\
\hline 11231232 & 3584 & 2005 Feb 22 & $043507.1+263429$ & $043502.8+264111$ & $39.1 \times 155.1$ & 173.2 & $10.4 \mathrm{HDR}$ \\
\hline 11232768 & 3584 & 2005 Feb 22 & $042625.5+271214$ & $042620.6+271854$ & $34.2 \times 184.2$ & 172.4 & $10.4 \mathrm{HDR}$ \\
\hline 11234560 & 3584 & 2005 Feb 22 & $043918.8+243141$ & $043914.7+243823$ & $48.7 \times 121.4$ & 173.3 & $10.4 \mathrm{HDR}$ \\
\hline 11235072 & 3584 & 2005 Feb 22 & $043507.1+263429$ & $043502.8+264111$ & $39.1 \times 155.1$ & 173.2 & $10.4 \mathrm{HDR}$ \\
\hline 11236608 & 3584 & 2005 Feb 22 & $042625.5+271214$ & $042620.6+271854$ & $34.2 \times 184.2$ & 172.4 & 10.4 HDR \\
\hline \multirow{10}{*}{3964672} & 37 & 2005 Feb 23 & $043051.5+244220$ & $043051.5+244220$ & $5.3 \times 5.3$ & 82.0 & $31.2 \mathrm{HDR}$ \\
\hline & & & $043056.4+243542$ & $043046.9+244903$ & $5.3 \times 5.3$ & 82.0 & $31.2 \mathrm{HDR}$ \\
\hline & & & $042907.5+244349$ & $042907.5+244349$ & $5.3 \times 5.3$ & 82.0 & $31.2 \mathrm{HDR}$ \\
\hline & & & $042913.2+243701$ & $042902.8+245032$ & $5.3 \times 5.3$ & 82.0 & $31.2 \mathrm{HDR}$ \\
\hline & & & $042924.4+243256$ & $042924.4+243256$ & $5.3 \times 5.3$ & 82.0 & $31.2 \mathrm{HDR}$ \\
\hline & & & $042928.4+242624$ & $042919.6+243938$ & $5.3 \times 5.3$ & 82.0 & $31.2 \mathrm{HDR}$ \\
\hline & & & $042656.4+244334$ & $042656.4+244334$ & $5.3 \times 5.3$ & 82.0 & $31.2 \mathrm{HDR}$ \\
\hline & & & $042701.2+243647$ & $042651.6+245016$ & $5.3 \times 5.3$ & 82.0 & $31.2 \mathrm{HDR}$ \\
\hline & & & $042339.3+245613$ & $042339.3+245613$ & $5.3 \times 5.3$ & 82.0 & $31.2 \mathrm{HDR}$ \\
\hline & & & $042344.5+244927$ & $042334.3+250255$ & $5.3 \times 5.3$ & 82.0 & $31.2 \mathrm{HDR}$ \\
\hline 11230208 & 3584 & 2005 Feb 23 & $044759.0+245848$ & $044755.6+250532$ & $63.3 \times 174.3$ & 174.6 & 10.4 HDR \\
\hline 11230464 & 3584 & 2005 Feb 23 & $044318.2+250731$ & $044314.5+251413$ & $58.5 \times 174.4$ & 174.0 & $10.4 \mathrm{HDR}$ \\
\hline 11230976 & 3584 & 2005 Feb 23 & $043821.2+263059$ & $043817.1+263742$ & $48.7 \times 121.3$ & 173.1 & $10.4 \mathrm{HDR}$ \\
\hline 11232256 & 3584 & 2005 Feb 23 & $042901.8+271637$ & $042857.2+272325$ & $34.2 \times 189.0$ & 172.6 & $10.4 \mathrm{HDR}$ \\
\hline 11234304 & 3584 & 2005 Feb 23 & $044318.2+250731$ & $044314.5+251413$ & $58.5 \times 174.4$ & 174.0 & $10.4 \mathrm{HDR}$ \\
\hline 11234816 & 3584 & 2005 Feb 23 & $043821.2+263059$ & $043817.1+263742$ & $48.7 \times 121.3$ & 173.1 & $10.4 \mathrm{HDR}$ \\
\hline 11231744 & 3584 & 2005 Feb 24 & $043139.4+272058$ & $043134.8+272743$ & $39.1 \times 198.8$ & 172.6 & $10.4 \mathrm{HDR}$ \\
\hline 11232512 & 3584 & 2005 Feb 24 & $043043.1+241804$ & $043038.4+242447$ & $34.4 \times 184.2$ & 172.9 & $10.4 \mathrm{HDR}$ \\
\hline 11234048 & 3584 & 2005 Feb 24 & $044759.0+245848$ & $044755.6+250532$ & $63.3 \times 174.3$ & 174.6 & $10.4 \mathrm{HDR}$ \\
\hline 11235584 & 3584 & 2005 Feb 24 & $043139.4+272058$ & $043134.8+272743$ & $39.1 \times 198.8$ & 172.6 & $10.4 \mathrm{HDR}$ \\
\hline 11236096 & 3584 & 2005 Feb 24 & $042901.8+271637$ & $042857.2+272325$ & $34.2 \times 189.0$ & 172.6 & $10.4 \mathrm{HDR}$ \\
\hline 11236352 & 3584 & 2005 Feb 24 & $043043.1+241804$ & $043038.4+242447$ & $34.4 \times 184.2$ & 172.9 & $10.4 \mathrm{HDR}$ \\
\hline \multirow{2}{*}{14604544} & 20386 & 2005 Sep 16 & $042336.8+264012$ & $042336.8+264012$ & $5.3 \times 5.3$ & 81.0 & $214.4 \mathrm{HDR}$ \\
\hline & & & $042331.1+264651$ & $042342.3+263331$ & $5.3 \times 5.3$ & 81.0 & $214.4 \mathrm{HDR}$ \\
\hline \multirow[t]{2}{*}{14604800} & 20386 & 2005 Sep 17 & $042336.8+264012$ & $042336.8+264012$ & $5.3 \times 5.3$ & 81.0 & $214.4 \mathrm{HDR}$ \\
\hline & & & $042331.1+264651$ & $042342.3+263331$ & $5.3 \times 5.3$ & 81.0 & $214.4 \mathrm{HDR}$ \\
\hline \multirow[t]{2}{*}{14617856} & 20386 & 2005 Sep 17 & $042156.7+152954$ & $042156.7+152954$ & $5.3 \times 5.3$ & 82.0 & $214.4 \mathrm{HDR}$ \\
\hline & & & $042152.0+153633$ & $042201.4+152309$ & & 82.0 & $214.4 \mathrm{HDR}$ \\
\hline \multirow[t]{2}{*}{14618112} & 20386 & 2005 Sep 17 & $042156.7+152954$ & $042156.7+152954$ & $5.3 \times 5.3$ & 82.0 & $214.4 \mathrm{HDR}$ \\
\hline & & & $042152.0+153633$ & $042201.4+152309$ & $5.3 \times 5.3$ & 82.0 & $214.4 \mathrm{HDR}$ \\
\hline \multirow[t]{2}{*}{15123456} & 20762 & 2006 Mar 23 & $043110.0+181236$ & $043110.0+181236$ & $5.5 \times 5.5$ & 82.7 & 241.2 \\
\hline & & & $043114.5+180557$ & $043105.7+181919$ & $5.5 \times 5.5$ & 82.7 & 241.2 \\
\hline 14605568 & 20386 & 2006 Mar 24 & $042839.2+265139$ & $042839.2+265139$ & $5.3 \times 5.3$ & 81.5 & $214.4 \mathrm{HDR}$ \\
\hline & & & $042844.6+264501$ & $042833.8+265822$ & $5.3 \times 5.3$ & 81.5 & $214.4 \mathrm{HDR}$ \\
\hline 14609920 & 20386 & 2006 Mar 24 & $042921.3+261425$ & $042921.3+261425$ & $5.3 \times 5.3$ & 81.5 & $214.4 \mathrm{HDR}$ \\
\hline & & & $042926.5+260746$ & $042916.0+262107$ & $5.3 \times 5.3$ & 81.5 & $214.4 \mathrm{HDR}$ \\
\hline 14511616 & 20302 & 2006 Mar 25 & $043509.5+240544$ & $043504.7+241228$ & $20.3 \times 32.6$ & 172.7 & 72.8 \\
\hline 14605824 & 20386 & 2006 Mar 25 & $042839.2+265139$ & $042839.2+265139$ & $5.3 \times 5.3$ & 81.5 & $214.4 \mathrm{HDR}$ \\
\hline & & & $042844.6+264501$ & $042833.8+265822$ & $5.3 \times 5.3$ & 81.5 & $214.4 \mathrm{HDR}$ \\
\hline 14610176 & 20386 & 2006 Mar 25 & $042921.3+261425$ & $042921.3+261425$ & $5.3 \times 5.3$ & 81.5 & $214.4 \mathrm{HDR}$ \\
\hline & & & $042926.5+260746$ & $042916.0+262107$ & $5.3 \times 5.3$ & 81.5 & $214.4 \mathrm{HDR}$ \\
\hline 14611968 & 20386 & 2006 Mar 25 & $043249.0+242518$ & $043249.0+242518$ & $5.3 \times 5.3$ & 82.4 & $214.4 \mathrm{HDR}$ \\
\hline & & & $043253.8+241835$ & $043244.0+243159$ & $5.3 \times 5.3$ & 82.4 & $214.4 \mathrm{HDR}$ \\
\hline 14612224 & 20386 & 2006 Mar 26 & $043249.0+242518$ & $043249.0+242518$ & $5.3 \times 5.3$ & 82.4 & $214.4 \mathrm{HDR}$ \\
\hline & & & $043253.8+241835$ & $043244.0+243159$ & & 82.4 & $214.4 \mathrm{HDR}$ \\
\hline 14610432 & 20386 & 2006 Mar 29 & $050416.8+251053$ & $050416.8+251053$ & $5.3 \times 5.3$ & 85.5 & $214.4 \mathrm{HDR}$ \\
\hline & & & $050420.1+250409$ & $050413.6+251740$ & $5.3 \times 5.3$ & 85.5 & $214.4 \mathrm{HDR}$ \\
\hline 14610688 & 20386 & 2006 Mar 29 & $050416.8+251053$ & $050416.8+251053$ & $5.3 \times 5.3$ & 85.5 & $214.4 \mathrm{HDR}$ \\
\hline & & & $050420.1+250409$ & $050413.6+251740$ & $5.3 \times 5.3$ & 85.5 & $214.4 \mathrm{HDR}$ \\
\hline 18365440 & 30540 & 2006 Sep 28 & $042216.0+254913$ & $042216.0+254913$ & $5.5 \times 5.5$ & 81.0 & 52 \\
\hline & & & $042210.5+255549$ & $042221.6+254233$ & $5.5 \times 5.5$ & 81.0 & 52 \\
\hline 18365696 & 30540 & 2006 Sep 28 & $041523.7+291046$ & $041523.7+291046$ & $5.5 \times 5.5$ & 79.5 & 134 \\
\hline & & & $041517.3+291719$ & $041530.1+290405$ & $5.5 \times 5.5$ & 79.5 & 134 \\
\hline 18363648 & 30540 & 2006 Oct 26 & $045748.5+301520$ & $045748.5+301520$ & $5.5 \times 5.5$ & 81.5 & 134 \\
\hline & & & $045743.3+302200$ & $045753.9+300840$ & $5.5 \times 5.5$ & 81.5 & 134 \\
\hline
\end{tabular}


TABLE 1 - Continued

\begin{tabular}{|c|c|c|c|c|c|c|c|}
\hline AOR & PID & $\begin{array}{l}\text { Date } \\
\text { (UT) }\end{array}$ & $\begin{array}{c}\text { 3.6/5.8 Center } \\
(\mathrm{J} 2000)\end{array}$ & $\begin{array}{c}\text { 4.5/8.0 Center } \\
(\mathrm{J} 2000)\end{array}$ & $\begin{array}{l}\text { Dimensions } \\
(\text { arcmin })\end{array}$ & $\begin{array}{c}\text { Angle }^{\mathrm{a}} \\
(\mathrm{deg})\end{array}$ & $\operatorname{Exp}_{(\mathrm{s})} \operatorname{Time}^{\mathrm{b}}$ \\
\hline \multirow[t]{2}{*}{18365952} & 30540 & 2006 Oct 26 & $044841.5+170341$ & $044841.5+170341$ & $5.5 \times 5.5$ & 87.5 & 134 \\
\hline & & & $044839.5+171022$ & $044843.5+165650$ & $5.5 \times 5.5$ & 87.5 & 134 \\
\hline \multirow{2}{*}{18363904} & 30540 & 2007 Mar 28 & $042630.8+244359$ & $042630.8+244359$ & $5.5 \times 5.5$ & 81.5 & 134 \\
\hline & & & $042636.1+243718$ & $042625.8+245036$ & $5.5 \times 5.5$ & 81.5 & 134 \\
\hline \multirow[t]{2}{*}{18364160} & 30540 & 2007 Mar 28 & $044145.2+230150$ & $044145.2+230150$ & $5.5 \times 5.5$ & 83.3 & 134 \\
\hline & & & $044149.5+225513$ & $044140.9+230836$ & $5.5 \times 5.5$ & 83.3 & 134 \\
\hline \multirow[t]{2}{*}{18364416} & 30540 & 2007 Mar 28 & $042154.9+265234$ & $042154.9+265234$ & $5.5 \times 5.5$ & 81.0 & 134 \\
\hline & & & $042200.5+264556$ & $042149.3+265912$ & $5.5 \times 5.5$ & 81.0 & 134 \\
\hline \multirow[t]{2}{*}{18364672} & 30540 & 2007 Mar 28 & $043610.7+215936$ & $043610.7+215936$ & $5.5 \times 5.5$ & 82.5 & 134 \\
\hline & & & $043615.2+215257$ & $043606.2+220618$ & $5.5 \times 5.5$ & 82.5 & 134 \\
\hline \multirow[t]{2}{*}{18364928} & 30540 & 2007 Mar 28 & $041433.5+303341$ & $041433.5+303341$ & $5.5 \times 5.5$ & 80.0 & 134 \\
\hline & & & $041439.8+302708$ & $041427.0+304020$ & $5.5 \times 5.5$ & 80.0 & 134 \\
\hline 19030528 & 30816 & 2007 Mar 28 & $041849.9+250250$ & $041843.8+250931$ & $36.7 \times 185.0$ & 170.3 & 10.4 HDR \\
\hline \multirow[t]{2}{*}{18365184} & 30540 & 2007 Mar 29 & $042213.7+193442$ & $042213.7+193442$ & $5.5 \times 5.5$ & 81.5 & 52 \\
\hline & & & $042218.7+192803$ & $042208.8+194122$ & $5.5 \times 5.5$ & 81.5 & 52 \\
\hline \multirow[t]{2}{*}{18366208} & 30540 & 2007 Mar 29 & $045523.7+302738$ & $045523.7+302738$ & $5.5 \times 5.5$ & 85.0 & 52 \\
\hline & & & $045527.3+302056$ & $045520.1+303423$ & $5.5 \times 5.5$ & 85.0 & 52 \\
\hline 18366464 & 30540 & 2007 Mar 29 & $043309.9+224649$ & $043309.9+224649$ & $5.5 \times 5.5$ & 82.5 & 52 \\
\hline & & & $043314.4+224012$ & $043305.1+225333$ & $5.5 \times 5.5$ & 82.5 & 52 \\
\hline 19027712 & 30816 & 2007 Mar 29 & $041908.8+290747$ & $041902.9+291426$ & $51.2 \times 80.2$ & 170.2 & 10.4 HDR \\
\hline 19027968 & 30816 & 2007 Mar 29 & $041908.8+290747$ & $041902.9+291426$ & $51.2 \times 80.2$ & 170.2 & $10.4 \mathrm{HDR}$ \\
\hline 19028736 & 30816 & 2007 Mar 29 & $042303.9+233420$ & $042258.6+234101$ & $51.2 \times 118.3$ & 170.9 & $10.4 \mathrm{HDR}$ \\
\hline 19030784 & 30816 & 2007 Mar 29 & $041346.3+281103$ & $041339.8+281742$ & $36.7 \times 186.7$ & 169.9 & $10.4 \mathrm{HDR}$ \\
\hline 19031040 & 30816 & 2007 Mar 29 & $041605.7+251253$ & $041600.0+251934$ & $36.7 \times 185.0$ & 170.2 & $10.4 \mathrm{HDR}$ \\
\hline 19034112 & 30816 & 2007 Mar 29 & $041849.9+250250$ & $041843.8+250931$ & $36.7 \times 185.0$ & 170.3 & $10.4 \mathrm{HDR}$ \\
\hline 19034368 & 30816 & 2007 Mar 29 & $041346.3+281103$ & $041339.8+281742$ & $36.7 \times 186.7$ & 169.9 & $10.4 \mathrm{HDR}$ \\
\hline 19028224 & 30816 & 2007 Mar 30 & $042142.1+253221$ & $042136.6+253901$ & $51.2 \times 117.3$ & 170.8 & $10.4 \mathrm{HDR}$ \\
\hline 19028480 & 30816 & 2007 Mar 30 & $042142.1+253221$ & $042136.6+253901$ & $51.2 \times 117.3$ & 170.8 & $10.4 \mathrm{HDR}$ \\
\hline 19028992 & 30816 & 2007 Mar 30 & $042303.9+233420$ & $042258.6+234101$ & $51.2 \times 118.3$ & 170.9 & $10.4 \mathrm{HDR}$ \\
\hline 19030272 & 30816 & 2007 Mar 30 & $041635.2+280108$ & $041628.8+280750$ & $36.7 \times 186.7$ & 169.9 & $10.4 \mathrm{HDR}$ \\
\hline 19034624 & 30816 & 2007 Mar 30 & $041605.7+251253$ & $041600.0+251934$ & $36.7 \times 185.0$ & 170.2 & $10.4 \mathrm{HDR}$ \\
\hline 19033856 & 30816 & 2007 Mar 31 & $041635.2+280108$ & $041628.8+280750$ & $36.7 \times 186.7$ & 169.9 & $10.4 \mathrm{HDR}$ \\
\hline 19032576 & 30816 & 2007 Apr 2 & $043536.6+221456$ & $043532.2+222140$ & $94.7 \times 109.3$ & 82.2 & $10.4 \mathrm{HDR}$ \\
\hline 19036160 & 30816 & 2007 Apr 3 & $043536.6+221456$ & $043532.2+222140$ & $94.7 \times 109.3$ & 82.2 & $10.4 \mathrm{HDR}$ \\
\hline 21916416 & 40302 & 2007 Oct 16 & $042024.9+270038$ & $042024.9+270038$ & $5.5 \times 5.5$ & 79.5 & 52 \\
\hline & & & $042018.8+270713$ & $042031.3+265358$ & $5.5 \times 5.5$ & 79.5 & 52 \\
\hline 24242688 & 462 & 2007 Oct 16 & $042216.8+243325$ & $042222.4+242644$ & $5.2 \times 51.0$ & 81.0 & $20.8 \mathrm{HDR}$ \\
\hline 24243200 & 462 & 2007 Oct 16 & $042042.5+265559$ & $042049.0+264922$ & $56.0 \times 56.0$ & 79.9 & $10.4 \mathrm{HDR}$ \\
\hline 24243456 & 462 & 2007 Oct 16 & $042042.5+265559$ & $042049.0+264922$ & $56.0 \times 56.0$ & 79.9 & $10.4 \mathrm{HDR}$ \\
\hline 24243712 & 462 & 2007 Oct 16 & $044527.0+251343$ & $044531.3+250700$ & $5.2 \times 162.4$ & 173.0 & $20.8 \mathrm{HDR}$ \\
\hline 24243968 & 462 & 2007 Oct 16 & $044113.5+243310$ & $044118.1+242627$ & $5.2 \times 87.3$ & 172.8 & $20.8 \mathrm{HDR}$ \\
\hline 24244224 & 462 & 2007 Oct 16 & $044033.0+260009$ & $044037.7+255327$ & $5.2 \times 87.3$ & 172.3 & $20.8 \mathrm{HDR}$ \\
\hline 24244480 & 462 & 2007 Oct 16 & $043410.9+280723$ & $043416.4+280042$ & $10.0 \times 41.4$ & 80.9 & $20.8 \mathrm{HDR}$ \\
\hline 24244736 & 462 & 2007 Oct 16 & $043439.0+242925$ & $043444.0+242243$ & $5.2 \times 152.7$ & 172.0 & $20.8 \mathrm{HDR}$ \\
\hline 24244992 & 462 & 2007 Oct 16 & $043322.5+265539$ & $043327.8+264858$ & $5.2 \times 152.7$ & 171.2 & $20.8 \mathrm{HDR}$ \\
\hline 24245248 & 462 & 2007 Oct 16 & $042917.2+242751$ & $042922.5+242110$ & $5.2 \times 181.7$ & 171.4 & $20.8 \mathrm{HDR}$ \\
\hline 24245504 & 462 & 2007 Oct 16 & $042739.0+272010$ & $042744.8+271330$ & $5.2 \times 181.7$ & 170.4 & $20.8 \mathrm{HDR}$ \\
\hline 24245760 & 462 & 2007 Oct 16 & $042641.6+242458$ & $042647.0+241817$ & $5.2 \times 181.7$ & 171.1 & $20.8 \mathrm{HDR}$ \\
\hline 24246016 & 462 & 2007 Oct 16 & $042457.8+272229$ & $042504.0+271549$ & $5.2 \times 181.7$ & 170.0 & $20.8 \mathrm{HDR}$ \\
\hline 24246272 & 462 & 2007 Oct 16 & $042157.7+273952$ & $042204.0+273311$ & $5.2 \times 46.2$ & 169.7 & $20.8 \mathrm{HDR}$ \\
\hline 24246528 & 462 & 2007 Oct 16 & $042132.2+282439$ & $042138.8+281800$ & $5.2 \times 46.2$ & 169.3 & $20.8 \mathrm{HDR}$ \\
\hline 24246784 & 462 & 2007 Oct 16 & $043418.8+275356$ & $043424.4+274714$ & $10.0 \times 41.4$ & 81.1 & $20.8 \mathrm{HDR}$ \\
\hline 24242944 & 462 & 2007 Oct 23 & $042207.1+244719$ & $042212.9+244038$ & $5.2 \times 51.0$ & 80.5 & $20.8 \mathrm{HDR}$ \\
\hline 26472448 & 50584 & 2008 Oct 31 & $041627.1+205257$ & $041627.1+205257$ & $6.0 \times 6.0$ & 81.3 & 41.6 HDR \\
\hline & & & $041623.9+205957$ & $041632.3+204620$ & $6.0 \times 6.0$ & 81.3 & $41.6 \mathrm{HDR}$ \\
\hline 26475008 & 50584 & 2008 Oct 31 & $042707.2+221455$ & $042707.2+221455$ & $6.0 \times 6.0$ & 82.0 & 41.6 HDR \\
\hline & & & $042700.0+222155$ & $042712.4+220810$ & $6.0 \times 6.0$ & 82.0 & 41.6 HDR \\
\hline & & & $042644.9+213152$ & $042644.9+213152$ & $6.0 \times 6.0$ & 82.0 & 41.6 HDR \\
\hline & & & $042712.4+220810$ & $042649.6+212510$ & $6.0 \times 6.0$ & 82.0 & $41.6 \mathrm{HDR}$ \\
\hline 26476800 & 50584 & 2008 Oct 31 & $050237.4+215418$ & $050237.4+215418$ & $6.0 \times 6.0$ & 86.0 & $41.6 \mathrm{HDR}$ \\
\hline & & & $050236.6+220056$ & $050240.5+214728$ & $6.0 \times 6.0$ & 86.0 & 41.6 HDR \\
\hline & & & $050646.5+210420$ & $050646.5+210420$ & $6.0 \times 6.0$ & 86.0 & 41.6 HDR \\
\hline & & & $050641.6+211139$ & $050649.4+205724$ & $6.0 \times 6.0$ & 86.0 & 41.6 HDR \\
\hline 26476544 & 50584 & 2008 Nov 1 & $045545.1+303610$ & $045549.7+302938$ & $18.0 \times 9.5$ & 82.5 & 41.6 HDR \\
\hline 26472960 & 50584 & 2008 Nov 2 & $041655.8+314049$ & $041655.8+314049$ & $6.0 \times 6.0$ & 77.0 & $41.6 \mathrm{HDR}$ \\
\hline & & & $041647.4+314710$ & $041704.2+313416$ & $6.0 \times 6.0$ & 77.0 & $41.6 \mathrm{HDR}$ \\
\hline & & & $042427.7+310340$ & $042427.7+310340$ & $6.0 \times 6.0$ & 77.0 & 41.6 HDR \\
\hline & & & $042421.2+311009$ & $042435.5+305708$ & $6.0 \times 6.0$ & 77.0 & $41.6 \mathrm{HDR}$ \\
\hline & & & $042117.3+305150$ & $042117.3+305150$ & $6.0 \times 6.0$ & 77.0 & $41.6 \mathrm{HDR}$ \\
\hline & & & $042435.5+305708$ & $042125.1+304523$ & $6.0 \times 6.0$ & 77.0 & 41.6 HDR \\
\hline & & & $041630.5+303715$ & $041630.5+303715$ & $6.0 \times 6.0$ & 77.0 & $41.6 \mathrm{HDR}$ \\
\hline & & & $042125.1+304523$ & $041638.3+303042$ & $6.0 \times 6.0$ & 77.0 & 41.6 HDR \\
\hline 26475520 & 50584 & 2008 Nov 2 & $044053.4+205544$ & $044053.4+205544$ & $6.0 \times 6.0$ & 84.0 & $41.6 \mathrm{HDR}$ \\
\hline & & & $044051.6+210239$ & $044057.0+204904$ & $6.0 \times 6.0$ & 84.0 & $41.6 \mathrm{HDR}$ \\
\hline
\end{tabular}


TABLE 1 - Continued

\begin{tabular}{|c|c|c|c|c|c|c|c|}
\hline AOR & PID & $\begin{array}{l}\text { Date } \\
\text { (UT) }\end{array}$ & $\begin{array}{c}\text { 3.6/5.8 Center } \\
(\mathrm{J} 2000)\end{array}$ & $\begin{array}{l}\text { 4.5/8.0 Center } \\
\text { (J2000) }\end{array}$ & $\begin{array}{l}\text { Dimensions } \\
\text { (arcmin) }\end{array}$ & $\begin{array}{l}\text { Angle } \\
(\mathrm{deg})\end{array}$ & $\operatorname{Exp}_{(\mathrm{s})} \operatorname{Time}^{\mathrm{b}}$ \\
\hline
\end{tabular}

a Position angle of the long axis of the map.

b Total exposure time for each position and filter. "HDR" indicates that $0.4 \mathrm{~s}$ exposures were also obtained.

TABLE 2

MIPS $24 \mu \mathrm{M}$ OBSERVING LOG

\begin{tabular}{|c|c|c|c|c|c|c|}
\hline AOR & PID & $\begin{array}{l}\text { Date } \\
\text { (UT) }\end{array}$ & $\begin{array}{l}\text { Center } \\
(\mathrm{J} 2000)\end{array}$ & $\begin{array}{l}\text { Dimensions } \\
(\text { arcmin })\end{array}$ & $\begin{array}{c}\text { Angle }^{\mathrm{a}} \\
(\mathrm{deg})\end{array}$ & $\begin{array}{l}\text { Exp Time } \\
(\mathrm{s})\end{array}$ \\
\hline 3662080 & 6 & 2004 Feb 20 & $043140.6+180845$ & $30.5 \times 54$ & 170.7 & 40 \\
\hline \multirow{2}{*}{5675776} & 173 & 2004 Sep 18 & $042024.3+312326$ & $7.4 \times 8.2$ & 167.9 & 277 \\
\hline & & & $042007.3+313521$ & $5.6 \times 8$ & 167.9 & 357 \\
\hline \multirow[t]{2}{*}{5680384} & 173 & 2004 Sep 18 & $043334.6+242109$ & $7.4 \times 8.2$ & 171.0 & 36 \\
\hline & & & $043321.6+243315$ & $5.6 \times 8$ & 171.0 & 31 \\
\hline \multirow[t]{2}{*}{5675264} & 173 & 2004 Sep 19 & $041739.0+283303$ & $7.4 \times 8.2$ & 168.2 & 73 \\
\hline & & & $041722.8+284500$ & $5.6 \times 8$ & 168.2 & 357 \\
\hline \multirow[t]{2}{*}{5676032} & 173 & 2004 Sep 19 & $042158.9+281807$ & $7.4 \times 8.2$ & 168.8 & 36 \\
\hline & & & $042143.3+283006$ & $5.6 \times 8$ & 168.8 & 344 \\
\hline \multirow{2}{*}{5676288} & 173 & 2004 Sep 19 & $042203.2+282540$ & $7.4 \times 8.2$ & 168.8 & 34 \\
\hline & & & $042147.6+283739$ & $5.6 \times 8$ & 168.8 & 357 \\
\hline \multirow[t]{2}{*}{9426432} & 139 & 2004 Sep 19 & $042334.7+263732$ & $5.6 \times 10.8$ & 169.3 & 26 \\
\hline & & & $042317.5+265159$ & $6 \times 10$ & 169.3 & 80 \\
\hline \multirow[t]{2}{*}{5673728} & 173 & 2004 Sep 22 & $040519.7+200926$ & $7.4 \times 8.2$ & 169.3 & 36 \\
\hline & & & $040505.5+202127$ & $5.6 \times 8$ & 169.3 & 357 \\
\hline \multirow[t]{2}{*}{5674240} & 173 & 2004 Sep 22 & $040917.2+171610$ & $7.4 \times 8.2$ & 170.7 & 73 \\
\hline & & & $040904.5+172814$ & $5.6 \times 8$ & 170.7 & 357 \\
\hline \multirow[t]{2}{*}{9422848} & 139 & 2004 Sep 22 & $042343.5+263748$ & $5.6 \times 10.8$ & 169.2 & 26 \\
\hline & & & $042326.1+265215$ & $6 \times 10$ & 169.2 & 80 \\
\hline 3662336 & 6 & 2004 Sep 23 & $041837.0+282213$ & $32.5 \times 56$ & 168.5 & 80 \\
\hline 3662592 & 6 & 2004 Sep 23 & $041632.9+281958$ & $32.5 \times 54$ & 168.3 & 80 \\
\hline \multirow[t]{4}{*}{5678848} & 173 & 2004 Sep 23 & $043209.4+175724$ & $7.4 \times 8.2$ & 172.6 & 110 \\
\hline & & & $043158.3+180935$ & $5.6 \times 8$ & 172.6 & 357 \\
\hline & & & $043534.2+175142$ & $7.4 \times 8.2$ & 172.9 & 110 \\
\hline & & & $043523.5+180354$ & $5.6 \times 8$ & 172.9 & 357 \\
\hline 12026368 & 53 & 2004 Sep 23 & $044327.5+294254$ & $13.4 \times 55.0$ & 171.4 & 161 \\
\hline 12026624 & 53 & 2004 Sep 23 & $041049.6+251153$ & $16.0 \times 55.0$ & 168.5 & 161 \\
\hline \multirow[t]{2}{*}{9409536} & 139 & 2004 Sep 24 & $042932.9+265619$ & $5.6 \times 10.8$ & 169.6 & 26 \\
\hline & & & $042916.0+271047$ & $6 \times 10$ & 169.6 & 80 \\
\hline \multirow[t]{2}{*}{9415168} & 139 & 2004 Sep 24 & $044053.6+260903$ & $7.4 \times 18.2$ & 171.2 & 36 \\
\hline & & & $044040.6+262110$ & $5.6 \times 13$ & 171.2 & 200 \\
\hline \multirow[t]{2}{*}{9416448} & 139 & 2004 Sep 24 & $044238.0+294510$ & $7.4 \times 18.2$ & 170.6 & 36 \\
\hline & & & $044223.9+295714$ & $5.6 \times 13$ & 170.6 & 200 \\
\hline 9431040 & 139 & 2004 Sep 24 & $042834.9+265215$ & $7.4 \times 18.2$ & 169.5 & 36 \\
\hline & & & $042820.2+270416$ & $5.6 \times 13$ & 169.5 & 200 \\
\hline 5673984 & 173 & 2004 Sep 25 & $040531.0+215112$ & $7.4 \times 8.2$ & 168.7 & 415 \\
\hline & & & $040516.1+220310$ & $5.6 \times 8$ & 168.7 & 357 \\
\hline 5674752 & 173 & 2004 Sep 25 & $041250.8+193659$ & $7.4 \times 8.2$ & 170.4 & 110 \\
\hline & & & $041237.6+194903$ & $5.6 \times 8$ & 170.4 & 357 \\
\hline 5675520 & 173 & 2004 Sep 25 & $041851.7+172316$ & $7.4 \times 8.2$ & 171.8 & 110 \\
\hline & & & $041840.0+173525$ & $5.6 \times 8$ & 171.8 & 357 \\
\hline 5676544 & 173 & 2004 Sep 25 & $042448.7+264314$ & $7.4 \times 8.2$ & 169.1 & 36 \\
\hline & & & $042433.6+265514$ & $5.6 \times 8$ & 169.1 & 119 \\
\hline 5676800 & 173 & 2004 Sep 25 & $042710.7+175043$ & $7.4 \times 8.2$ & 172.4 & 36 \\
\hline & & & $042659.4+180253$ & $5.6 \times 8$ & 172.4 & 357 \\
\hline 5677056 & 173 & 2004 Sep 25 & $042942.1+263256$ & $7.4 \times 8.2$ & 169.8 & 36 \\
\hline & & & $042927.7+264457$ & $5.6 \times 8$ & 169.8 & 119 \\
\hline 5677312 & 173 & 2004 Sep 25 & $043004.1+181350$ & $7.4 \times 8.2$ & 172.5 & 36 \\
\hline & & & $042952.9+182600$ & $5.6 \times 8$ & 172.5 & 357 \\
\hline 5677824 & 173 & 2004 Sep 25 & $043051.5+244224$ & $7.4 \times 8.2$ & 170.5 & 73 \\
\hline & & & $043037.9+245428$ & $5.6 \times 8$ & 170.5 & 94 \\
\hline 5678080 & 173 & 2004 Sep 25 & $043122.6+241056$ & $7.4 \times 8.2$ & 170.7 & 73 \\
\hline & & & $043109.3+242300$ & $5.6 \times 8$ & 170.7 & 357 \\
\hline 5678336 & 173 & 2004 Sep 25 & $043127.3+170626$ & $7.4 \times 8.2$ & 173.0 & 277 \\
\hline & & & $043116.6+171838$ & $5.6 \times 8$ & 173.0 & 357 \\
\hline 5678592 & 173 & 2004 Sep 25 & $043157.8+182138$ & $7.4 \times 8.2$ & 172.6 & 219 \\
\hline & & & $043150.7+182357$ & $5.6 \times 8$ & 172.6 & 312 \\
\hline 5679104 & 173 & 2004 Sep 25 & $043218.9+242229$ & $7.4 \times 8.2$ & 170.7 & 36 \\
\hline & & & $043205.6+243434$ & $5.6 \times 8$ & 170.7 & 357 \\
\hline 5679360 & 173 & 2004 Sep 25 & $043243.9+180259$ & $7.4 \times 8.2$ & 172.8 & 139 \\
\hline & & & $043233.0+181510$ & $5.6 \times 8$ & 172.8 & 357 \\
\hline 5679616 & 173 & 2004 Sep 25 & $043253.4+173535$ & $7.4 \times 8.2$ & 172.9 & 73 \\
\hline & & & $043242.7+174747$ & $5.6 \times 8$ & 172.9 & 357 \\
\hline 5680896 & 173 & 2004 Sep 25 & $043619.2+254300$ & $7.4 \times 8.2$ & 170.8 & 73 \\
\hline & & & $043605.8+255506$ & $5.6 \times 8$ & 170.8 & 357 \\
\hline
\end{tabular}


TABLE $2-$ Continued

\begin{tabular}{|c|c|c|c|c|c|c|}
\hline AOR & PID & $\begin{array}{l}\text { Date } \\
\text { (UT) }\end{array}$ & $\begin{array}{c}\text { Center } \\
(\mathrm{J} 2000)\end{array}$ & $\begin{array}{c}\text { Dimensions } \\
(\text { arcmin })\end{array}$ & $\begin{array}{c}\text { Angle }^{\mathrm{a}} \\
(\mathrm{deg})\end{array}$ & $\begin{array}{l}\text { Exp Time } \\
(\mathrm{s})\end{array}$ \\
\hline \multirow[t]{2}{*}{5682432} & 173 & 2004 Sep 25 & $044205.6+252258$ & $7.4 \times 8.2$ & 171.6 & 36 \\
\hline & & & $044153.0+253506$ & $5.6 \times 8$ & 171.6 & 357 \\
\hline \multirow[t]{2}{*}{5683200} & 173 & 2004 Sep 25 & $045602.3+302104$ & $7.4 \times 8.2$ & 172.1 & 36 \\
\hline & & & $045549.6+303313$ & $5.6 \times 8$ & 172.1 & 357 \\
\hline \multirow[t]{2}{*}{5684480} & 173 & 2004 Sep 25 & $050306.7+252321$ & $7.4 \times 8.2$ & 173.9 & 73 \\
\hline & & & $050256.3+253535$ & $5.6 \times 8$ & 173.9 & 357 \\
\hline \multirow[t]{2}{*}{9413376} & 139 & 2004 Sep 25 & $044136.6+260129$ & $7.4 \times 18.2$ & 171.3 & 36 \\
\hline & & & $044123.7+261336$ & $5.6 \times 13$ & 171.3 & 200 \\
\hline \multirow[t]{2}{*}{9413888} & 139 & 2004 Sep 25 & $042941.7+265635$ & $5.6 \times 10.8$ & 169.6 & 26 \\
\hline & & & $042924.8+271103$ & $6 \times 10$ & 169.6 & 80 \\
\hline \multirow{2}{*}{9414144} & 139 & 2004 Sep 25 & $043242.9+244947$ & $5.6 \times 10.8$ & 170.6 & 26 \\
\hline & & & $043227.4+250419$ & $6 \times 10$ & 170.6 & 80 \\
\hline \multirow[t]{2}{*}{9415424} & 139 & 2004 Sep 25 & $044129.6+260118$ & $7.4 \times 18.2$ & 171.3 & 36 \\
\hline & & & $044116.7+261324$ & $5.6 \times 13$ & 171.3 & 200 \\
\hline 9417984 & 139 & 2004 Sep 25 & $044147.6+254229$ & $7.4 \times 18.2$ & 171.4 & 36 \\
\hline & & & $044134.8+255436$ & $5.6 \times 13$ & 171.4 & 200 \\
\hline 9419776 & 139 & 2004 Sep 25 & $042842.1+265228$ & $7.4 \times 18.2$ & 169.5 & 36 \\
\hline & & & $042827.4+270429$ & $5.6 \times 13$ & 169.5 & 200 \\
\hline 9425152 & 139 & 2004 Sep 25 & $044100.6+260916$ & $7.4 \times 18.2$ & 171.2 & 36 \\
\hline & & & $044047.6+262122$ & $5.6 \times 13$ & 171.2 & 200 \\
\hline 9425920 & 139 & 2004 Sep 25 & $043004.4+242318$ & $5.6 \times 10.8$ & 170.5 & 26 \\
\hline & & & $042948.8+243750$ & $6 \times 10$ & 170.5 & 80 \\
\hline 9427712 & 139 & 2004 Sep 25 & $043245.3+242407$ & $7.4 \times 18.2$ & 170.8 & 36 \\
\hline 9428992 & 139 & 2004 Sep 25 & $044113.6+254935$ & $7.4 \times 18.2$ & 171.3 & 36 \\
\hline & & & $044100.7+260142$ & $5.6 \times 13$ & 171.3 & 200 \\
\hline 9430528 & 139 & 2004 Sep 25 & $044239.4+294223$ & $7.4 \times 18.2$ & 170.5 & 36 \\
\hline & & & $044225.2+295427$ & $5.6 \times 13$ & 170.5 & 200 \\
\hline 9432832 & 139 & 2004 Sep 25 & $043252.3+242420$ & $7.4 \times 18.2$ & 170.8 & 36 \\
\hline & & & $043239.1+243625$ & $5.6 \times 13$ & 170.8 & 200 \\
\hline 9434624 & 139 & 2004 Sep 25 & $044106.6+254924$ & $7.4 \times 18.2$ & 171.3 & 36 \\
\hline & & & $044053.7+260130$ & $5.6 \times 13$ & 171.3 & 200 \\
\hline 9434880 & 139 & 2004 Sep 25 & $043234.1+244930$ & $5.6 \times 10.8$ & 170.6 & 26 \\
\hline & & & $043218.6+250402$ & $6 \times 10$ & & 80 \\
\hline 9435136 & 139 & 2004 Sep 25 & $044140.6+254218$ & $7.4 \times 18.2$ & 171.4 & 36 \\
\hline & & & $044127.8+255425$ & $5.6 \times 13$ & 171.4 & 200 \\
\hline 9435392 & 139 & 2004 Sep 25 & $043152.8+243239$ & $7.4 \times 18.2$ & 170.6 & 36 \\
\hline & & & $043139.4+244443$ & $5.6 \times 13$ & 170.6 & 200 \\
\hline 9439488 & 139 & 2004 Sep 25 & $043159.8+243252$ & $7.4 \times 18.2$ & 170.6 & 36 \\
\hline & & & $043146.4+244456$ & $5.6 \times 13$ & 170.6 & 200 \\
\hline 9410816 & 139 & 2004 Sep 26 & $043013.2+242335$ & $5.6 \times 10.8$ & 170.5 & 26 \\
\hline & & & $042957.6+243806$ & $6 \times 10$ & 170.5 & 80 \\
\hline 5682944 & 173 & 2004 Oct 12 & $045537.1+301756$ & $7.4 \times 8.2$ & 169.9 & 36 \\
\hline & & & $045522.3+302958$ & $5.6 \times 8$ & 169.9 & 357 \\
\hline 5683712 & 173 & 2004 Oct 13 & $045730.7+201430$ & $7.4 \times 8.2$ & 175.6 & 73 \\
\hline & & & $045722.2+202648$ & $5.6 \times 8$ & 175.6 & 357 \\
\hline 5683968 & 173 & 2004 Oct 13 & $045839.9+204645$ & $7.4 \times 8.2$ & 175.4 & 400 \\
\hline & & & $045831.2+205902$ & & 175.4 & 357 \\
\hline 5681152 & 173 & 2005 Feb 25 & $043726.7+185124$ & $7.4 \times 8.2$ & 170.6 & 73 \\
\hline & & & $043739.6+183920$ & & 170.6 & 357 \\
\hline 5705984 & 173 & 2005 Feb 25 & $043812.9+202245$ & $7.4 \times 8.2$ & 171.0 & 139 \\
\hline & & & $043825.5+201040$ & $5.6 \times 8$ & 171.0 & 357 \\
\hline 5673472 & 173 & 2005 Feb 26 & $040349.1+261052$ & $7.4 \times 8.2$ & 168.9 & 139 \\
\hline & & & $040404.2+255854$ & $5.6 \times 8$ & 168.9 & 357 \\
\hline 5675008 & 173 & 2005 Feb 26 & $041447.9+275233$ & $7.4 \times 8.2$ & 170.4 & 36 \\
\hline & & & $041501.9+274030$ & $5.6 \times 8$ & 170.4 & 357 \\
\hline & & & $041314.0+281909$ & $7.4 \times 8.2$ & 170.3 & 36 \\
\hline & & & $041328.2+280706$ & $5.6 \times 8$ & 170.3 & 357 \\
\hline & & & $041941.2+274938$ & $7.4 \times 8.2$ & 170.9 & 36 \\
\hline & & & $041954.7+273732$ & $5.6 \times 8$ & 170.9 & 357 \\
\hline 5677568 & 173 & 2005 Feb 26 & $043029.5+242644$ & $7.4 \times 8.2$ & 171.3 & 292 \\
\hline & & & $043037.2+242428$ & & & 312 \\
\hline 5679872 & 173 & 2005 Feb 26 & $043306.5+240944$ & $7.4 \times 8.2$ & 171.5 & 36 \\
\hline & & & $043319.0+235736$ & $5.6 \times 8$ & 171.5 & 357 \\
\hline 5680128 & 173 & 2005 Feb 26 & $043309.9+243342$ & $7.4 \times 8.2$ & 171.6 & 36 \\
\hline & & & $043322.4+242134$ & $5.6 \times 8$ & 171.6 & 357 \\
\hline & & & $043439.2+250100$ & & & 36 \\
\hline & & & $043451.5+244851$ & $5.6 \times 8$ & 171.9 & 357 \\
\hline 5680640 & 173 & 2005 Feb 26 & $043556.8+235204$ & $7.4 \times 8.2$ & 171.7 & 73 \\
\hline & & & $043609.1+233956$ & & 171.7 & 357 \\
\hline 5681920 & 173 & 2005 Feb 26 & $044104.6+245106$ & $7.4 \times 8.2$ & 172.5 & 36 \\
\hline & & & $044116.3+243856$ & & 172.5 & 357 \\
\hline 5682176 & 173 & 2005 Feb 26 & $044108.3+255606$ & $7.4 \times 8.2$ & 172.7 & 73 \\
\hline & & & $044119.8+254355$ & & & 110 \\
\hline & & & $043835.1+261038$ & $7.4 \times 8.2$ & 172.5 & 73 \\
\hline & & & $043846.9+255827$ & $5.6 \times 8$ & 172.5 & 120 \\
\hline 5682688 & 173 & 2005 Feb 26 & $045230.7+173025$ & $7.4 \times 8.2$ & 171.3 & 36 \\
\hline
\end{tabular}


TABLE $2-$ Continued

\begin{tabular}{|c|c|c|c|c|c|c|}
\hline AOR & PID & $\begin{array}{l}\text { Date } \\
\text { (UT) }\end{array}$ & $\begin{array}{l}\text { Center } \\
(\mathrm{J} 2000)\end{array}$ & $\begin{array}{l}\text { Dimensions } \\
(\text { arcmin })\end{array}$ & $\begin{array}{c}\text { Angle }^{\mathrm{a}} \\
(\mathrm{deg})\end{array}$ & $\begin{array}{l}\text { Exp Time } \\
(\mathrm{s})\end{array}$ \\
\hline & & & $045242.8+171818$ & $5.6 \times 8$ & 171.3 & 357 \\
\hline & & & $045250.1+162209$ & $7.4 \times 8.2$ & 171.0 & 36 \\
\hline & & & $045302.4+161003$ & $5.6 \times 8$ & 171.0 & 357 \\
\hline \multirow[t]{2}{*}{9412096} & 139 & 2005 Feb 26 & $043555.7+241207$ & $5.6 \times 10.8$ & 171.8 & 26 \\
\hline & & & $043609.8+235731$ & $6 \times 10$ & 171.8 & 80 \\
\hline \multirow[t]{2}{*}{9433856} & 139 & 2005 Feb 26 & $043547.1+241151$ & $5.6 \times 10.8$ & 171.8 & 26 \\
\hline & & & $043601.2+235715$ & $6 \times 10$ & 171.8 & 80 \\
\hline 11229184 & 3584 & 2005 Feb 27 & $042444.8+253718$ & $35.6 \times 385.3$ & 171.7 & 15.5 \\
\hline 11229440 & 3584 & 2005 Feb 27 & $042433.3+253636$ & $35.6 \times 385.3$ & 171.7 & 15.5 \\
\hline 12915200 & 3584 & 2005 Feb 27 & $043311.0+285712$ & $55.8 \times 85.2$ & 173.3 & 15.5 \\
\hline 12915456 & 3584 & 2005 Feb 27 & $043256.3+290313$ & $55.8 \times 85.2$ & 173.3 & 15.5 \\
\hline 11225600 & 3584 & 2005 Feb 28 & $044800.4+250129$ & $65.8 \times 186.3$ & 174.0 & 15.5 \\
\hline 11225856 & 3584 & 2005 Feb 28 & $044750.6+250050$ & $65.8 \times 186.3$ & 174.0 & 15.5 \\
\hline 11226112 & 3584 & 2005 Feb 28 & $044318.9+250917$ & $65.8 \times 186.3$ & 173.5 & 15.5 \\
\hline 11226368 & 3584 & 2005 Feb 28 & $044309.5+250903$ & $65.8 \times 186.3$ & 173.5 & 15.5 \\
\hline 11228672 & 3584 & 2005 Feb 28 & $042718.4+254206$ & $35.6 \times 385.3$ & 171.9 & 15.5 \\
\hline 11229696 & 3584 & 2005 Feb 28 & $041946.7+274916$ & $60.7 \times 85.2$ & 171.5 & 15.5 \\
\hline 11229952 & 3584 & 2005 Feb 28 & $041957.8+274922$ & $60.7 \times 85.2$ & 171.5 & 15.5 \\
\hline 3662848 & 6 & 2005 Mar 1 & $041428.6+281309$ & $32.5 \times 54$ & 170.8 & 80 \\
\hline 11227136 & 3584 & 2005 Mar 1 & $043539.5+252026$ & $40.6 \times 311.3$ & 172.7 & 15.5 \\
\hline 11227392 & 3584 & 2005 Mar 1 & $043528.4+251950$ & $40.6 \times 311.3$ & 172.7 & 15.5 \\
\hline 11227648 & 3584 & 2005 Mar 1 & $043227.8+255148$ & $35.6 \times 385.3$ & 172.4 & 15.5 \\
\hline 11228928 & 3584 & 2005 Mar 1 & $042708.0+254127$ & $35.6 \times 385.3$ & 171.9 & 15.5 \\
\hline 11227904 & 3584 & 2005 Mar 2 & $043217.7+255107$ & $35.6 \times 385.3$ & 172.4 & 15.5 \\
\hline 11228160 & 3584 & 2005 Mar 2 & $042953.7+254657$ & $35.6 \times 385.3$ & 172.1 & 15.5 \\
\hline 11228416 & 3584 & 2005 Mar 2 & $042943.2+254617$ & $35.6 \times 385.3$ & 172.1 & 15.5 \\
\hline \multirow[t]{4}{*}{5681408} & 173 & 2005 Mar 3 & $043838.9+154613$ & $7.4 \times 8.2$ & 170.6 & 34 \\
\hline & & & $043851.6+153408$ & & & 357 \\
\hline & & & $044551.2+155549$ & $7.4 \times 8.2$ & 171.1 & 36 \\
\hline & & & $044603.5+154343$ & $5.6 \times 8$ & 171.1 & 357 \\
\hline 11226624 & 3584 & 2005 Mar 4 & $043851.4+253239$ & $50.7 \times 253.8$ & 172.9 & 15.5 \\
\hline 11226880 & 3584 & 2005 Mar 5 & $043840.0+253202$ & $50.7 \times 253.8$ & 172.9 & 15.5 \\
\hline 12662784 & 37 & 2005 Mar & $045535.8+303505$ & $17.8 \times 56.0$ & 175.5 & 80 \\
\hline \multirow[t]{6}{*}{12663296} & 37 & 2005 Mar 6 & $040442.9+261853$ & & & 36 \\
\hline & & & $040458.4+260656$ & & & 31 \\
\hline & & & $040350.8+261050$ & $7.4 \times 8.2$ & 168.4 & 36 \\
\hline & & & $040406.5+255855$ & $5.6 \times 8.0$ & 168.4 & 31 \\
\hline & & & $040313.9+255256$ & $7.4 \times$ & & 36 \\
\hline & & & $040329.6+254101$ & $5.6 \times 8.0$ & 168 & 31 \\
\hline 14614016 & 20386 & 2005 Sep 25 & $043008.8+242329$ & $5.6 \times 1$ & 0.6 & 292 \\
\hline 14617344 & 20386 & 2005 Sep 25 & $043250.4+242247$ & $5.6 \times 1$ & 171.0 & 292 \\
\hline 14613760 & 20386 & 2005 Sep 26 & $042339.1+263742$ & $5.6 \times 1$ & 169.5 & 292 \\
\hline 14618368 & 20386 & 2005 Sep 26 & $042158.4+152720$ & 5.6 & 172.0 & 297 \\
\hline 14618624 & 20386 & 2005 Sep 26 & $042158.4+152730$ & $5.6 \times 10.8$ & 172.0 & 297 \\
\hline 12615168 & & 2006 Feb 19 & $043140.9+180844$ & $32.5 x$ & 169.3 & 80 \\
\hline 14511872 & 20302 & 2006 Feb 26 & $043509.4+240646$ & $25.7 \times 56.0$ & 172.5 & 200 \\
\hline 18966528 & 30765 & 2006 Oct 9 & $043137.3+243623$ & $5.6 \times 8.0$ & & 950 \\
\hline 19026944 & 30816 & 2007 Feb 23 & $041913.5+290736$ & $50.7 \times 85.2$ & 174.2 & 15.5 \\
\hline 19027456 & 30816 & 2007 Feb 23 & $041902.3+290705$ & $50.7 \times 85.2$ & 174.1 & 15.5 \\
\hline 19029248 & 30816 & 2007 Feb 23 & $041748.1+263211$ & $35.6>$ & 172.6 & 15.5 \\
\hline 19032832 & 30816 & 2007 Feb 23 & $041737.2+263136$ & $35.6 \times 385.3$ & 172.5 & 15.5 \\
\hline 14510592 & 20302 & 2007 Feb 24 & $042916.9+261141$ & $25.7 \times 56.0$ & 173.4 & 200 \\
\hline 19029504 & 30816 & 2007 Feb 26 & $041501.2+264211$ & $35.6 \times 385.3$ & 172.1 & 15.5 \\
\hline 19032320 & & 2007 Feb 26 & $043541.8+221449$ & & 171.7 & 15.5 \\
\hline 19033088 & 30816 & $2007 \mathrm{Fe}$ & $041450.3+264135$ & 35.6 & 172.0 & 15.5 \\
\hline 19035904 & 30816 & 2007 Feb 27 & $043531.3+221412$ & $111 \times 85.2$ & 171.7 & 15.5 \\
\hline 19026688 & 30816 & 2007 Feb 28 & $042220.6+244329$ & $50.7 \times 253.8$ & 171.9 & 15.5 \\
\hline 19027200 & 30816 & 2007 Feb 28 & $042203.6+245249$ & $50.7 \times 253.8$ & 171.8 & 15.5 \\
\hline 18152960 & 30384 & 2007 Sep 23 & $042155.3+153211$ & $14.7 \times 56.0$ & 170.4 & 161 \\
\hline 53728 & 30384 & $2007 \mathrm{Se}$ & $042335.3+264231$ & $14.7 \times 56.0$ & 171.3 & 161 \\
\hline 18153984 & 30384 & $2007 \mathrm{Se}$ & $043004.2+242738$ & & 171.8 & 161 \\
\hline 18154240 & 30384 & 2007 Sep 23 & $043156.2+243453$ & $14.7 \times 5$ & 172.0 & 161 \\
\hline 18154496 & 30384 & 2007 Sep 24 & $043237.6+242552$ & $14.7 \times 56.0$ & 172.0 & 161 \\
\hline 18155264 & 30384 & 2007 Sep 24 & $044108.8+254555$ & & 173.0 & 161 \\
\hline 18153216 & 30384 & 2007 Sep 25 & $042838.4+265400$ & $14.7 \times 56.0$ & 171.7 & 161 \\
\hline 18155008 & 30384 & 2007 Sep 25 & $044151.4+255015$ & $14.7 \times 56.0$ & 173.1 & 161 \\
\hline 18155520 & 30384 & 2007 Sep 25 & $044237.4+294610$ & $14.7 \times 56.0$ & & 161 \\
\hline 24248064 & 462 & 2007 Sep 27 & $043401.6+280012$ & $40.6 \times 85.2$ & 172.3 & 15.5 \\
\hline 24248320 & 462 & 2007 Sep 27 & $043412.8+280017$ & & 172.3 & 15.5 \\
\hline 24247552 & 462 & 2007 Sep 29 & $042006.0+245609$ & $10.5 \times 186.3$ & 170.6 & 15.5 \\
\hline 24247808 & 462 & 2007 Sep 29 & $042006.2+245553$ & $10.5 \times 186.3$ & 170.6 & 15.5 \\
\hline 24248576 & 462 & 2007 Oct 27 & $043357.1+250921$ & $7.8 \times 253.8$ & 171.0 & 15.5 \\
\hline 24247040 & 462 & 2007 Oct 28 & $042029.2+271522$ & $60.7 \times 85.2$ & 168.1 & 15.5 \\
\hline 24247296 & 462 & 2007 Oct 28 & $042040.3+271536$ & $60.7 \times 85.2$ & 168.1 & 15.5 \\
\hline 23273728 & 40844 & 2007 Oct 29 & $041958.6+283324$ & $5.6 \times$ & 167.7 & 594 \\
\hline 23273984 & 40844 & 2007 Oct 29 & $042008.3+283115$ & $5.6 \times 8.0$ & 166.7 & 475 \\
\hline
\end{tabular}


TABLE $2-$ Continued

\begin{tabular}{ccccccr}
\hline \hline AOR & PID & $\begin{array}{c}\text { Date } \\
\text { (UT) }\end{array}$ & $\begin{array}{c}\text { Center } \\
(\mathrm{J} 2000)\end{array}$ & $\begin{array}{c}\text { Dimensions } \\
(\operatorname{arcmin})\end{array}$ & $\begin{array}{c}\text { Angle }^{\mathrm{a}} \\
(\mathrm{deg})\end{array}$ & $\begin{array}{c}\text { Exp Time } \\
(\mathrm{s})\end{array}$ \\
\hline 23274240 & 40844 & 2007 Oct 29 & $042008.8+281601$ & $5.6 \times 8.0$ & 166.8 & 475 \\
23276544 & 40844 & 2007 Oct 29 & $042516.3+283318$ & $5.6 \times 8.0$ & 167.4 & 594 \\
23277056 & 40844 & 2007 Oct 29 & $042618.3+282740$ & $5.6 \times 8.0$ & 167.6 & 594 \\
18155776 & 30384 & 2008 Apr 17 & $050417.4+250826$ & $14.7 \times 56.0$ & 174.9 & 161 \\
& & & & & & \\
\hline
\end{tabular}

a Position angle of the long axis of the map.

TABLE 3

IRAC APERTURe Corrections

\begin{tabular}{lllll}
\hline \hline Band & $2 / 14 / 15^{\mathrm{a}}$ & $3 / 3 / 4^{\mathrm{a}}$ & $4 / 4 / 5^{\mathrm{a}}$ & $4 / 4 / 10^{\mathrm{a}}$ \\
\hline$[3.6]$ & 0.450 & 0.270 & $0.180^{\mathrm{b}}$ & $\cdots$ \\
{$[4.5]$} & 0.485 & 0.300 & $0.170^{\mathrm{b}}$ & $\cdots$ \\
{$[5.8]$} & 0.645 & 0.515 & $\cdots$ & $0.165^{\mathrm{b}}$ \\
{$[8.0]$} & 0.750 & 0.735 & $\cdots$ & $0.260^{\mathrm{b}}$ \\
& & & & \\
\hline
\end{tabular}

${ }^{a}$ Aperture radius and inner and outer radii of the sky annulus in pixels ( 1 pixel $=0$.' 86 ).

b Default aperture and sky annulus.

TABLE 4

IRAC Photometry for Members of Taurus

\begin{tabular}{|c|c|c|c|c|c|c|}
\hline $2 \mathrm{MASS}^{\mathrm{a}}$ & Name & {$[3.6]$} & {$[4.5]$} & {$[5.8]$} & {$[8.0]$} & Date \\
\hline J04034930+2610520 & HBC $358 \mathrm{~A}+\mathrm{B}+\mathrm{C}$ & $9.15 \pm 0.02$ & $9.11 \pm 0.02$ & $9.05 \pm 0.03$ & $9.05 \pm 0.03$ & 2005 Feb 20 \\
\hline J04034997+2620382 & XEST 06-006 & out & out & out & out & \\
\hline J04035084+2610531 & HBC 359 & $9.33 \pm 0.02$ & $9.26 \pm 0.02$ & $9.23 \pm 0.03$ & $9.23 \pm 0.03$ & 2005 Feb 20 \\
\hline J04043936+2158186 & HBC 360 & $9.71 \pm 0.02$ & $9.69 \pm 0.02$ & $9.62 \pm 0.03$ & $9.62 \pm 0.03$ & $2004 \mathrm{Feb} 11$ \\
\hline J04043984+2158215 & HBC 361 & $9.87 \pm 0.02$ & $9.81 \pm 0.02$ & $9.76 \pm 0.03$ & $9.76 \pm 0.03$ & 2004 Feb 11 \\
\hline J04044307+2618563 & IRAS $04016+2610$ & $6.76 \pm 0.02$ & $5.46 \pm 0.02$ & $4.42 \pm 0.03$ & $3.56 \pm 0.03$ & 2005 Feb 20 \\
\hline J04053087+2151106 & HBC 362 & $9.83 \pm 0.02$ & $9.82 \pm 0.02$ & $9.76 \pm 0.03$ & $9.75 \pm 0.03$ & 2005 Feb 22 \\
\hline J04080782+2807280 & & out & out & out & out & \\
\hline \multirow[t]{2}{*}{ J04131414+2819108 } & $\mathrm{LkCa} 1$ & $8.47 \pm 0.02$ & $8.47 \pm 0.02$ & $8.40 \pm 0.03$ & $8.40 \pm 0.03$ & 2005 Feb 20 \\
\hline & & $8.52 \pm 0.02$ & $8.45 \pm 0.02$ & $8.39 \pm 0.03$ & $8.44 \pm 0.03$ & 2007 Mar 29 \\
\hline \multirow{3}{*}{ J04132722+2816247 } & Anon 1 & $7.18 \pm 0.02$ & $7.16 \pm 0.02$ & $7.13 \pm 0.03$ & $7.07 \pm 0.03$ & 2005 Feb 19 \\
\hline & & $7.23 \pm 0.02$ & $7.18 \pm 0.02$ & $7.10 \pm 0.03$ & $7.07 \pm 0.03$ & 2005 Feb 20 \\
\hline & & $7.22 \pm 0.02$ & $7.18 \pm 0.02$ & $7.10 \pm 0.03$ & $7.07 \pm 0.03$ & 2007 Mar 29 \\
\hline \multirow{2}{*}{ J04135328+2811233 } & IRAS $04108+2803 \mathrm{~A}$ & $8.98 \pm 0.02$ & $8.34 \pm 0.02$ & $7.75 \pm 0.03$ & $6.70 \pm 0.03$ & 2005 Feb 19 \\
\hline & & $8.97 \pm 0.02$ & $8.34 \pm 0.02$ & $7.62 \pm 0.03$ & $6.52 \pm 0.03$ & 2007 Mar 29 \\
\hline \multirow[t]{2}{*}{$\mathrm{J} 04135471+2811328$} & IRAS $04108+2803 \mathrm{~B}$ & $9.28 \pm 0.02$ & $7.94 \pm 0.02$ & $6.81 \pm 0.03$ & $5.81 \pm 0.03$ & 2005 Feb 19 \\
\hline & & $9.32 \pm 0.02$ & $8.02 \pm 0.02$ & $7.08 \pm 0.03$ & $5.87 \pm 0.03$ & 2007 Mar 29 \\
\hline \multirow[t]{2}{*}{ J04135737+2918193 } & IRAS $04108+2910$ & $7.90 \pm 0.02$ & $7.26 \pm 0.02$ & $6.70 \pm 0.03$ & $5.96 \pm 0.03$ & 2005 Feb 20 \\
\hline & & $7.46 \pm 0.02$ & $6.74 \pm 0.02$ & $6.22 \pm 0.03$ & $5.49 \pm 0.03$ & 2007 Mar 29 \\
\hline \multirow[t]{2}{*}{ J04141188+2811535 } & $\ldots$ & $10.82 \pm 0.02$ & $10.27 \pm 0.02$ & $9.80 \pm 0.03$ & $8.89 \pm 0.03$ & 2005 Feb 19 \\
\hline & & & $10.35 \pm 0.02$ & $9.88 \pm 0.03$ & $8.99 \pm 0.03$ & 2007 Mar 29 \\
\hline \multirow[t]{2}{*}{$\cdots$} & IRAS $04111+2800 \mathrm{G}$ & $13.02 \pm 0.02$ & $11.82 \pm 0.02$ & $11.20 \pm 0.03$ & $10.49 \pm 0.03$ & 2005 Feb 19 \\
\hline & & $13.15 \pm 0.02$ & $11.84 \pm 0.02$ & $11.10 \pm 0.03$ & $10.36 \pm 0.03$ & 2007 Mar 29 \\
\hline \multirow{2}{*}{ J04141291+2812124 } & V773 Tau A+B & $6.00 \pm 0.02$ & $5.53 \pm 0.02$ & $5.08 \pm 0.03$ & $4.41 \pm 0.03$ & 2005 Feb 19 \\
\hline & & $6.00 \pm 0.02$ & $5.55 \pm 0.02$ & $5.05 \pm 0.03$ & $4.36 \pm 0.03$ & 2007 Mar 29 \\
\hline \multirow{2}{*}{ J04141358+2812492 } & FM Tau & $7.82 \pm 0.02$ & $7.40 \pm 0.02$ & $7.13 \pm 0.03$ & $6.22 \pm 0.03$ & 2005 Feb 19 \\
\hline & & $8.10 \pm 0.02$ & $7.63 \pm 0.02$ & $7.28 \pm 0.03$ & $6.39 \pm 0.03$ & 2007 Mar 29 \\
\hline \multirow{2}{*}{ J04141458+2827580 } & FN Tau & $7.46 \pm 0.02$ & $6.97 \pm 0.02$ & $6.53 \pm 0.03$ & $5.57 \pm 0.03$ & 2005 Feb 19 \\
\hline & & $7.55 \pm 0.02$ & $7.11 \pm 0.02$ & $6.64 \pm 0.03$ & $5.84 \pm 0.03$ & 2007 Mar 29 \\
\hline \multirow[t]{2}{*}{ J04141700+2810578 } & CW Tau & sat & $5.23 \pm 0.02$ & $4.79 \pm 0.03$ & $4.31 \pm 0.03$ & 2005 Feb 19 \\
\hline & & $5.86 \pm 0.02$ & & $4.98 \pm 0.03$ & $4.39 \pm 0.03$ & 2007 Mar 29 \\
\hline \multirow[t]{2}{*}{ J04141760+2806096 } & CIDA 1 & $8.48 \pm 0.02$ & $7.84 \pm 0.02$ & $7.40 \pm 0.03$ & $6.53 \pm 0.03$ & 2005 Feb 19 \\
\hline & & $8.64 \pm 0.02$ & $8.10 \pm 0.02$ & $7.59 \pm 0.03$ & $6.57 \pm 0.03$ & 2007 Mar 29 \\
\hline \multirow[t]{2}{*}{ J04142626+2806032 } & MHO 1 & $6.63 \pm 0.05$ & $5.78 \pm 0.05$ & $5.20 \pm 0.05$ & $4.52 \pm 0.05$ & 2005 Feb 19 \\
\hline & & $6.00 \pm 0.05$ & & & $3.90 \pm 0.05$ & 2007 Mar 29 \\
\hline \multirow[t]{2}{*}{ J04142639+2805597 } & MHO 2 & $7.21 \pm 0.05$ & $6.49 \pm 0.05$ & $5.87 \pm 0.05$ & $5.02 \pm 0.05$ & 2005 Feb 19 \\
\hline & & $7.50 \pm 0.05$ & $6.61 \pm 0.05$ & $5.92 \pm 0.05$ & $4.99 \pm 0.05$ & 2007 Mar 29 \\
\hline \multirow[t]{2}{*}{ J04143054+2805147 } & MHO 3 & $7.40 \pm 0.02$ & $6.84 \pm 0.02$ & $6.26 \pm 0.03$ & $5.11 \pm 0.03$ & 2005 Feb 19 \\
\hline & & $7.21 \pm 0.02$ & $6.44 \pm 0.02$ & $5.69 \pm 0.03$ & $4.57 \pm 0.03$ & 2007 Mar 29 \\
\hline \multirow{2}{*}{ J04144730+2646264 } & FP Tau & $8.47 \pm 0.02$ & $8.25 \pm 0.02$ & $7.86 \pm 0.03$ & $7.25 \pm 0.03$ & 2004 Feb 10 \\
\hline & & $8.07 \pm 0.02$ & $7.78 \pm 0.02$ & $7.58 \pm 0.03$ & $7.28 \pm 0.03$ & 2007 Mar 29 \\
\hline \multirow[t]{2}{*}{ J04144739+2803055 } & XEST 20-066 & $9.78 \pm 0.02$ & $9.55 \pm 0.02$ & $9.59 \pm 0.03$ & $9.53 \pm 0.03$ & 2005 Feb 19 \\
\hline & & $9.61 \pm 0.02$ & $9.58 \pm 0.02$ & $9.50 \pm 0.03$ & $9.50 \pm 0.03$ & 2007 Mar 29 \\
\hline \multirow{2}{*}{ J04144786+2648110 } & CX Tau & $8.41 \pm 0.02$ & $8.06 \pm 0.02$ & $7.62 \pm 0.03$ & $6.64 \pm 0.03$ & 2004 Feb 10 \\
\hline & & $8.51 \pm 0.02$ & $8.01 \pm 0.02$ & $7.64 \pm 0.03$ & $6.62 \pm 0.03$ & 2007 Mar 29 \\
\hline
\end{tabular}


TABLE 4 - Continued

\begin{tabular}{|c|c|c|c|c|c|c|}
\hline $2 \mathrm{MASS}^{\mathrm{a}}$ & Name & {$[3.6]$} & {$[4.5]$} & {$[5.8]$} & {$[8.0]$} & Date \\
\hline \multirow[t]{2}{*}{ J04144797+2752346 } & $\mathrm{LkCa} 3 \mathrm{~A}+\mathrm{B}$ & $7.35 \pm 0.02$ & $7.28 \pm 0.02$ & $7.24 \pm 0.03$ & $7.23 \pm 0.03$ & 2005 Feb 20 \\
\hline & & $7.26 \pm 0.02$ & $7.28 \pm 0.02$ & $7.22 \pm 0.03$ & $7.22 \pm 0.03$ & 2007 Mar 29 \\
\hline \multirow{2}{*}{ J04144928+2812305 } & FO Tau A+B & $7.66 \pm 0.02$ & $7.12 \pm 0.02$ & $6.81 \pm 0.03$ & $5.84 \pm 0.03$ & 2005 Feb 19 \\
\hline & & $7.47 \pm 0.02$ & $7.09 \pm 0.02$ & $6.72 \pm 0.03$ & $5.88 \pm 0.03$ & 2007 Mar 29 \\
\hline \multirow[t]{2}{*}{ J04145234+2805598 } & XEST 20-071 & $7.41 \pm 0.02$ & $7.34 \pm 0.02$ & $7.28 \pm 0.03$ & $7.24 \pm 0.03$ & 2005 Feb 19 \\
\hline & & $7.40 \pm 0.02$ & $7.36 \pm 0.02$ & $7.24 \pm 0.03$ & $7.21 \pm 0.03$ & 2007 Mar 29 \\
\hline \multirow[t]{3}{*}{ J04150515+2808462 } & CIDA 2 & $8.83 \pm 0.02$ & $8.74 \pm 0.02$ & $8.68 \pm 0.03$ & $8.70 \pm 0.03$ & 2005 Feb 19 \\
\hline & & out & $8.73 \pm 0.02$ & out & $8.64 \pm 0.03$ & 2005 Feb 20 \\
\hline & & $8.83 \pm 0.02$ & $8.69 \pm 0.02$ & $8.67 \pm 0.03$ & $8.78 \pm 0.03$ & 2007 Mar 29 \\
\hline \multirow[t]{3}{*}{ J04151471+2800096 } & KPNO 1 & $13.21 \pm 0.02$ & out & $13.03 \pm 0.05$ & out & 2005 Feb 19 \\
\hline & & $13.19 \pm 0.02$ & $13.06 \pm 0.02$ & $13.10 \pm 0.04$ & $12.81 \pm 0.06$ & 2005 Feb 20 \\
\hline & & $13.18 \pm 0.02$ & $13.04 \pm 0.02$ & $13.09 \pm 0.05$ & $13.09 \pm 0.07$ & 2007 Mar 29 \\
\hline \multirow[t]{2}{*}{ J04152409+2910434 } & $\cdots$ & $11.85 \pm 0.02$ & $11.71 \pm 0.02$ & $11.65 \pm 0.03$ & $11.60 \pm 0.03$ & 2006 Sep 28 \\
\hline & & $11.81 \pm 0.02$ & $11.71 \pm 0.02$ & $11.62 \pm 0.03$ & $11.50 \pm 0.04$ & 2007 Mar 29 \\
\hline \multirow[t]{2}{*}{ J04153916+2818586 } & $\cdots$ & $8.67 \pm 0.02$ & $8.38 \pm 0.02$ & $8.45 \pm 0.03$ & $7.53 \pm 0.03$ & 2005 Feb 19 \\
\hline & & $8.73 \pm 0.02$ & $8.43 \pm 0.02$ & $8.23 \pm 0.03$ & $7.53 \pm 0.03$ & 2007 Mar 29 \\
\hline J04154278+2909597 & IRAS $04125+2902$ & $9.09 \pm 0.02$ & $9.06 \pm 0.02$ & $8.97 \pm 0.03$ & $9.05 \pm 0.03$ & 2007 Mar 29 \\
\hline J04155799+2746175 & $\ldots$ & $9.71 \pm 0.02$ & $9.37 \pm 0.02$ & $9.04 \pm 0.03$ & $8.36 \pm 0.03$ & 2007 Mar 29 \\
\hline J04161210+2756385 & $\cdots$ & $9.35 \pm 0.02$ & $8.99 \pm 0.02$ & $8.69 \pm 0.03$ & $8.29 \pm 0.03$ & 2007 Mar 29 \\
\hline J04161885+2752155 & $\cdots$ & $10.92 \pm 0.02$ & $10.74 \pm 0.02$ & $10.64 \pm 0.03$ & $10.68 \pm 0.03$ & 2007 Mar 29 \\
\hline J04162725+2053091 & $\ldots$ & $10.77 \pm 0.02$ & $10.67 \pm 0.02$ & $10.65 \pm 0.03$ & $10.59 \pm 0.03$ & 2008 Oct 31 \\
\hline J04162810+2807358 & $\mathrm{LkCa} 4$ & $8.26 \pm 0.02$ & $8.06 \pm 0.02$ & $8.04 \pm 0.03$ & $7.97 \pm 0.03$ & 2005 Feb 19 \\
\hline & & $8.19 \pm 0.02$ & $8.17 \pm 0.02$ & $7.99 \pm 0.03$ & $8.03 \pm 0.03$ & 2007 Mar 29 \\
\hline J04163048+3037053 & $\ldots$ & $12.29 \pm 0.02$ & $12.17 \pm 0.02$ & $12.16 \pm 0.03$ & $12.23 \pm 0.04$ & 2008 Nov 2 \\
\hline $\mathrm{J} 04163911+2858491$ & $\ldots$ & $10.46 \pm 0.02$ & $10.07 \pm 0.02$ & $9.82 \pm 0.03$ & $9.41 \pm 0.03$ & 2007 Mar 29 \\
\hline J04173372+2820468 & CY Tau & $7.96 \pm 0.02$ & $7.62 \pm 0.02$ & $7.30 \pm 0.03$ & $6.74 \pm 0.03$ & 2005 Feb 19 \\
\hline & & $7.94 \pm 0.02$ & $7.71 \pm 0.02$ & $7.25 \pm 0.03$ & $6.69 \pm 0.03$ & 2005 Feb 21 \\
\hline & & $7.63 \pm 0.02$ & $7.25 \pm 0.02$ & $7.02 \pm 0.03$ & $6.48 \pm 0.03$ & 2007 Mar 29 \\
\hline J04173893+2833005 & LkCa 5 & out & $8.85 \pm 0.02$ & out & $8.76 \pm 0.03$ & 2005 Feb 19 \\
\hline & & $8.88 \pm 0.02$ & $8.84 \pm 0.02$ & $8.80 \pm 0.03$ & $8.76 \pm 0.03$ & 2005 Feb 20 \\
\hline & & $8.90 \pm 0.02$ & out & $8.78 \pm 0.03$ & out & 2007 Mar 29 \\
\hline J04174955+2813318 & KPNO 10 & $10.79 \pm 0.02$ & $10.32 \pm 0.02$ & $9.80 \pm 0.03$ & $8.84 \pm 0.03$ & 2005 Feb 19 \\
\hline & & $10.79 \pm 0.02$ & $10.29 \pm 0.02$ & $9.88 \pm 0.03$ & $8.81 \pm 0.03$ & 2005 Feb 21 \\
\hline & & $10.80 \pm 0.02$ & out & out & out & 2007 Mar 29 \\
\hline J04174965+2829362 & V410 X-ray 1 & $8.17 \pm 0.02$ & $7.73 \pm 0.02$ & $7.25 \pm 0.03$ & $6.42 \pm 0.03$ & 2005 Feb 19 \\
\hline & & out & $7.76 \pm 0.02$ & out & $6.42 \pm 0.03$ & 2005 Feb 21 \\
\hline & & $8.41 \pm 0.02$ & out & $7.39 \pm 0.03$ & out & 2007 Mar 29 \\
\hline J04180796+2826036 & V410 X-ray 3 & $9.99 \pm 0.02$ & $9.85 \pm 0.02$ & $9.86 \pm 0.03$ & $9.82 \pm 0.03$ & 2005 Feb 19 \\
\hline & & $9.96 \pm 0.02$ & $9.91 \pm 0.02$ & $9.88 \pm 0.03$ & $9.81 \pm 0.03$ & 2005 Feb 21 \\
\hline & & $9.99 \pm 0.02$ & out & $9.78 \pm 0.03$ & out & 2007 Mar 29 \\
\hline J04181078+2519574 & V409 Tau & $8.05 \pm 0.02$ & $7.70 \pm 0.02$ & $7.25 \pm 0.03$ & $6.30 \pm 0.03$ & 2007 Mar 30 \\
\hline $\mathrm{J} 04181710+2828419$ & V410 Anon 13 & $10.24 \pm 0.02$ & $9.81 \pm 0.02$ & $9.46 \pm 0.03$ & $8.81 \pm 0.03$ & 2005 Feb 19 \\
\hline & & out & $9.92 \pm 0.02$ & out & $8.80 \pm 0.03$ & 2005 Feb 21 \\
\hline & & $10.20 \pm 0.02$ & out & $9.47 \pm 0.03$ & out & 2007 Mar 29 \\
\hline J04182147+1658470 & HBC 372 & $10.39 \pm 0.02$ & $10.39 \pm 0.02$ & $10.40 \pm 0.03$ & $10.33 \pm 0.03$ & 2004 Mar 8 \\
\hline J04182909+2826191 & V410 Anon 25 & $8.87 \pm 0.02$ & $8.58 \pm 0.02$ & $8.38 \pm 0.03$ & $8.40 \pm 0.03$ & 2005 Feb 19 \\
\hline & & $8.89 \pm 0.02$ & $8.56 \pm 0.02$ & $8.41 \pm 0.03$ & $8.37 \pm 0.03$ & 2005 Feb 21 \\
\hline & & $8.92 \pm 0.02$ & out & $8.31 \pm 0.03$ & out & 2007 Mar 29 \\
\hline J04183030+2743208 & KPNO 11 & $10.66 \pm 0.02$ & $10.54 \pm 0.02$ & $10.55 \pm 0.03$ & $10.49 \pm 0.03$ & 2005 Feb 21 \\
\hline J04183110+2827162 & V410 Tau A+B+C & $7.40 \pm 0.02$ & $7.29 \pm 0.02$ & $7.31 \pm 0.03$ & $7.20 \pm 0.03$ & 2005 Feb 19 \\
\hline & & $7.31 \pm 0.02$ & $7.31 \pm 0.02$ & $7.34 \pm 0.03$ & $7.24 \pm 0.03$ & 2005 Feb 21 \\
\hline & & $7.37 \pm 0.02$ & out & $7.29 \pm 0.03$ & out & 2007 Mar 29 \\
\hline J04183112+2816290 & DD Tau A+B & $6.54 \pm 0.02$ & $5.72 \pm 0.02$ & $5.19 \pm 0.03$ & $4.41 \pm 0.03$ & 2005 Feb 19 \\
\hline & & $6.45 \pm 0.02$ & $5.78 \pm 0.02$ & $5.23 \pm 0.03$ & $4.42 \pm 0.03$ & 2005 Feb 21 \\
\hline J04183158+2816585 & CZ Tau A+B & $8.47 \pm 0.02$ & $7.60 \pm 0.02$ & $6.57 \pm 0.03$ & $5.00 \pm 0.03$ & 2005 Feb 19 \\
\hline & & $8.46 \pm 0.02$ & $7.55 \pm 0.02$ & $6.63 \pm 0.03$ & $4.95 \pm 0.03$ & 2005 Feb 21 \\
\hline J04183203+2831153 & IRAS $04154+2823$ & $7.89 \pm 0.02$ & $7.03 \pm 0.02$ & $6.31 \pm 0.03$ & $5.51 \pm 0.03$ & 2005 Feb 19 \\
\hline & & out & $6.99 \pm 0.02$ & out & $5.47 \pm 0.03$ & 2005 Feb 21 \\
\hline & & $7.55 \pm 0.02$ & out & $6.06 \pm 0.03$ & out & 2007 Mar 29 \\
\hline J04183444+2830302 & V410 X-ray 2 & $8.22 \pm 0.02$ & $8.06 \pm 0.02$ & $7.84 \pm 0.03$ & $7.50 \pm 0.03$ & 2005 Feb 19 \\
\hline & & out & $8.04 \pm 0.02$ & out & $7.66 \pm 0.03$ & 2005 Feb 21 \\
\hline & & $8.30 \pm 0.02$ & out & $7.77 \pm 0.03$ & out & 2007 Mar 29 \\
\hline J04184023+2824245 & V410 X-ray 4 & $8.83 \pm 0.02$ & $8.64 \pm 0.02$ & $8.47 \pm 0.03$ & $8.41 \pm 0.03$ & 2005 Feb 19 \\
\hline & & $8.85 \pm 0.02$ & $8.58 \pm 0.02$ & $8.55 \pm 0.03$ & $8.41 \pm 0.03$ & 2005 Feb 21 \\
\hline J04184061+2819155 & V892 Tau & sat & sat & $3.56 \pm 0.03$ & sat & 2005 Feb 19 \\
\hline & & & & $3.56 \pm 0.03$ & & 2005 Feb 21 \\
\hline J04184133+2827250 & LR1 & $9.38 \pm 0.02$ & $8.86 \pm 0.02$ & $8.40 \pm 0.03$ & $7.95 \pm 0.03$ & 2005 Feb 19 \\
\hline & & $9.45 \pm 0.02$ & $8.82 \pm 0.02$ & $8.42 \pm 0.03$ & $7.97 \pm 0.03$ & 2005 Feb 21 \\
\hline & & $9.38 \pm 0.02$ & out & $8.35 \pm 0.03$ & out & 2007 Mar 29 \\
\hline J04184250+2818498 & V410 X-ray 7 & $8.74 \pm 0.02$ & $8.59 \pm 0.02$ & $8.38 \pm 0.03$ & $8.08 \pm 0.04$ & 2005 Feb 19 \\
\hline & & & & & $8.04 \pm 0.04$ & 2005 Feb 21 \\
\hline J04184703+2820073 & Hubble 4 & $7.16 \pm 0.02$ & $7.08 \pm 0.02$ & $6.94 \pm 0.03$ & $6.92 \pm 0.03$ & 2005 Feb 19 \\
\hline & & & $7.01 \pm 0.02$ & $6.98 \pm 0.03$ & $6.94 \pm 0.03$ & 2005 Feb 21 \\
\hline J04185115+2814332 & KPNO 2 & $12.19 \pm 0.02$ & $12.09 \pm 0.02$ & $12.04 \pm 0.04$ & $12.02 \pm 0.04$ & 2005 Feb 19 \\
\hline & & $12.21 \pm 0.02$ & $12.07 \pm 0.02$ & $11.96 \pm 0.04$ & $11.76 \pm 0.04$ & 2005 Feb 21 \\
\hline J04185147+2820264 & CoKu Tau/1 & $10.07 \pm 0.02$ & $8.85 \pm 0.02$ & $7.56 \pm 0.03$ & $5.71 \pm 0.03$ & 2005 Feb 19 \\
\hline & & $10.19 \pm 0.02$ & $8.96 \pm 0.02$ & $7.72 \pm 0.03$ & $5.84 \pm 0.03$ & 2005 Feb 21 \\
\hline
\end{tabular}


TABLE 4 - Continued

\begin{tabular}{|c|c|c|c|c|c|c|}
\hline $2 \mathrm{MASS}^{\mathrm{a}}$ & Name & {$[3.6]$} & {$[4.5]$} & {$[5.8]$} & {$[8.0]$} & Date \\
\hline J04185170+1723165 & HBC 376 & $9.24 \pm 0.02$ & $9.20 \pm 0.02$ & $9.16 \pm 0.03$ & $9.13 \pm 0.03$ & 2004 Sep 7 \\
\hline \multirow{2}{*}{ J04185813+2812234 } & IRAS $04158+2805$ & $9.21 \pm 0.02$ & $8.46 \pm 0.02$ & $7.74 \pm 0.03$ & $6.83 \pm 0.03$ & 2005 Feb 19 \\
\hline & & $9.26 \pm 0.02$ & $8.43 \pm 0.02$ & $7.78 \pm 0.03$ & $6.92 \pm 0.03$ & 2005 Feb 21 \\
\hline \multirow[t]{2}{*}{ J04190110+2819420 } & V410 X-ray 6 & $8.73 \pm 0.02$ & $8.60 \pm 0.02$ & $8.53 \pm 0.03$ & $8.25 \pm 0.03$ & 2005 Feb 19 \\
\hline & & $8.85 \pm 0.02$ & $8.59 \pm 0.02$ & $8.49 \pm 0.03$ & $8.25 \pm 0.03$ & 2005 Feb 21 \\
\hline \multirow[t]{3}{*}{ J04190126+2802487 } & KPNO 12 & $13.89 \pm 0.02$ & out & $13.05 \pm 0.05$ & out & 2005 Feb 19 \\
\hline & & $13.94 \pm 0.02$ & $13.49 \pm 0.02$ & $13.11 \pm 0.04$ & $12.56 \pm 0.05$ & 2005 Feb 20 \\
\hline & & $13.93 \pm 0.02$ & $13.54 \pm 0.03$ & $13.17 \pm 0.05$ & $12.66 \pm 0.05$ & 2005 Feb 21 \\
\hline \multirow[t]{2}{*}{ J04190197+2822332 } & V410 X-ray 5a & $9.64 \pm 0.02$ & $9.50 \pm 0.02$ & $9.48 \pm 0.03$ & $9.40 \pm 0.03$ & 2005 Feb 19 \\
\hline & & $9.65 \pm 0.02$ & $9.58 \pm 0.02$ & bad & $9.48 \pm 0.03$ & 2005 Feb 21 \\
\hline \multirow[t]{3}{*}{ J04191281+2829330 } & FQ Tau A+B & $8.69 \pm 0.02$ & $8.34 \pm 0.02$ & $8.03 \pm 0.03$ & $7.39 \pm 0.03$ & 2005 Feb 19 \\
\hline & & out & $8.35 \pm 0.02$ & out & $7.42 \pm 0.03$ & 2005 Feb 21 \\
\hline & & $8.72 \pm 0.02$ & $\begin{array}{l}\text { out } \\
\text { out }\end{array}$ & $8.09 \pm 0.03$ & out & 2007 Mar 29 \\
\hline \multirow[t]{2}{*}{ J04191583+2906269 } & BP Tau & $7.33 \pm 0.02$ & $6.97 \pm 0.02$ & $6.67 \pm 0.03$ & $5.66 \pm 0.03$ & 2005 Feb 20 \\
\hline & & $7.23 \pm 0.02$ & $6.84 \pm 0.02$ & $6.61 \pm 0.03$ & $5.66 \pm 0.03$ & 2007 Mar 29 \\
\hline \multirow[t]{2}{*}{ J04192625+2826142 } & V819 Tau & $8.19 \pm 0.02$ & $8.16 \pm 0.02$ & $8.06 \pm 0.03$ & $8.06 \pm 0.03$ & 2005 Feb 19 \\
\hline & & $8.20 \pm 0.02$ & $8.24 \pm 0.02$ & $8.13 \pm 0.03$ & $8.04 \pm 0.03$ & 2005 Feb 21 \\
\hline J04193545+2827218 & FR Tau & $9.39 \pm 0.02$ & $8.84 \pm 0.02$ & $8.23 \pm 0.03$ & $7.25 \pm 0.03$ & 2005 Feb 19 \\
\hline & & $9.43 \pm 0.02$ & $8.87 \pm 0.02$ & $8.32 \pm 0.03$ & $7.26 \pm 0.03$ & 2005 Feb 21 \\
\hline J04194127+2749484 & $\mathrm{LkCa} 7 \mathrm{~A}+\mathrm{B}$ & $8.06 \pm 0.02$ & $8.03 \pm 0.02$ & $7.99 \pm 0.03$ & $7.94 \pm 0.03$ & 2005 Feb 20 \\
\hline & & $8.05 \pm 0.02$ & $8.05 \pm 0.02$ & $8.01 \pm 0.03$ & $7.96 \pm 0.03$ & 2005 Feb 21 \\
\hline J04194148+2716070 & IRAS $04166+2708$ & $11.13 \pm 0.02$ & $10.31 \pm 0.02$ & $9.91 \pm 0.03$ & $9.24 \pm 0.03$ & 2005 Feb 20 \\
\hline & & $11.25 \pm 0.02$ & $10.36 \pm 0.02$ & $9.92 \pm$ & $9.31 \pm 0.03$ & 2005 Feb 21 \\
\hline & & $11.19 \pm 0.02$ & out & $9.75 \pm 0.03$ & out & 2007 Oct 17 \\
\hline & IRAS $04166+2706$ & & $11.01 \pm 0.03$ & $10.22 \pm 0.03$ & $9.59 \pm 0.03$ & 2005 Feb 20 \\
\hline & & $\ldots$ & $11.03 \pm 0.03$ & $10.40 \pm 0.03$ & $9.67 \pm 0.03$ & 2005 Feb 21 \\
\hline & & & $11.26 \pm 0.03$ & $10.43=$ & $9.90 \pm 0.03$ & 2007 Oct 17 \\
\hline J04194657+2712552 & [GKH94] 41 & $10.03 \pm 0.02$ & $9.20 \pm 0.02$ & $8.60 \pm 0.03$ & $8.03 \pm 0.03$ & 2005 Feb 20 \\
\hline & & $9.99 \pm 0.02$ & out & $8.60 \pm 0.03$ & out & 2005 Feb 21 \\
\hline & & $10.27=$ & $9.40 \pm 0.02$ & 0.03 & $8.16 \pm 0.03$ & 2007 Oct 17 \\
\hline J04195844+2709570 & IRAS $04169+2702$ & $8.45 \pm 0.02$ & $7.20 \pm 0.02$ & \pm 0.03 & $5.36 \pm 0.03$ & 2005 Feb 20 \\
\hline & & $8.43 \pm 0.02$ & out & $6.32 \pm 0.03$ & out & 2005 Feb 21 \\
\hline & & $8.31 \pm 0.02$ & $7.08 \pm 0.02$ & 0.03 & $5.28 \pm 0.03$ & 2007 Oct 17 \\
\hline $\mathrm{J} 04201611+2821325$ & $\ldots$ & $11.81 \pm 0.02$ & $11.40 \pm 0.02$ & 11.11 & $10.56 \pm 0.03$ & 2005 Feb 21 \\
\hline J042021 & & $12.71 \pm$ & $12.29 \pm 0.02$ & 0.04 & $11.56 \pm 0.04$ & 2005 Feb 21 \\
\hline J04202555+2700355 & & $10.95 \pm 0.02$ & $10.71 \pm 0.02$ & $10.44 \pm 0.03$ & $9.73 \pm 0.03$ & 2007 Oct 16 \\
\hline & & 10.93 & $10.68 \pm 0.02$ & 10.43 & $9.75=$ & 2007 Oct 17 \\
\hline J04202583+2819237 & IRAS $04173+2812$ & $9.18 \pm 0.02$ & $8.40 \pm 0.02$ & 0.03 & $7.13 \pm 0.03$ & 2005 Feb 21 \\
\hline & & $9.29=$ & 0.02 & & $7.03 \pm 0.03$ & $2005 \mathrm{Fe}$ \\
\hline & XEST 16-045 & $9.36=$ & $9.44 \pm 0.02$ & 0.03 & $9.31 \pm 0.03$ & 2005 Feb 21 \\
\hline & & $9.40 \pm 0.02$ & out & $9.31 \pm 0.03$ & out & 2007 Oct 17 \\
\hline J04205273+1746415 & $\mathrm{J} 2-157$ & $10.44 \pm 0.02$ & $10.35 \pm 0.02$ & 10.31 & $10.28 \pm 0.03$ & 2004 Mar 8 \\
\hline J04210795+2702204 & & $8.10=$ & $7.28 \pm 0.02$ & $6.63=$ & $5.54 \pm 0.03$ & 2005 Feb 20 \\
\hline & & 7.58 & 0.02 & & 5.12 & $2007 \mathrm{O}$ \\
\hline J04210934+2750368 & & $10.00 \pm 0.02$ & $9.72 \pm 0.02$ & $=0.03$ & $9.20 \pm 0.03$ & 2005 Feb 21 \\
\hline J04211038+2701372 & IRAS $04181+2654 \mathrm{~B}$ & 9.0 & 0.02 & 7.6 & $6.92=$ & 2005 Feb 20 \\
\hline & & & 0.02 & 7.60 & 6.76 & 2007 Oct 17 \\
\hline J04211146+2701094 & IRAS $04181+2654 \mathrm{~A}$ & $8.39 \pm 0.02$ & $7.36 \pm 0.02$ & $6.48 \pm 0.03$ & $5.46 \pm 0.03$ & 2005 Feb 20 \\
\hline & & & 0.02 & & $5.67 \pm 0.03$ & 2007 Oct 17 \\
\hline J04213459+2701388 & & $9.91 \pm 0.02$ & $9.61 \pm 0.02$ & $=0.03$ & $8.98 \pm 0.03$ & 2007 Oct 17 \\
\hline & XEST & $10.63 \pm 0.02$ & $10.58 \pm 0.02$ & 10.54 & $10.52 \pm 0.03$ & 2007 Oct 16 \\
\hline J04214323+1934133 & IRA & $6.96 \pm 0.02$ & $6.29 \pm 0.02$ & $5.53 \pm 0.03$ & $4.36=$ & 2005 Feb 20 \\
\hline J04214631+2659296 & & out & $11.24 \pm 0.02$ & out & $10.42 \pm 0.03$ & 2007 Mar 28 \\
\hline & & $3 \pm 0.02$ & $11.27 \pm 0.02$ & \pm 0.03 & $10.58 \pm 0.03$ & 2007 Oct 17 \\
\hline J04215450+2652315 & $\cdots$ & \pm 0.02 & out & \pm 0.05 & out & 2005 Feb 20 \\
\hline & & & $5 \pm 0.02$ & & $12.94 \pm 0.04$ & 2007 Mar 28 \\
\hline & & \pm 0.02 & $13.07 \pm 0.02$ & $12.88 \pm 0.04$ & $12.84 \pm 0.06$ & 2007 Oct 17 \\
\hline J04215563+2755060 & DE Tau & $7.02 \pm 0.02$ & $6.67 \pm 0.02$ & $6.38 \pm 0.03$ & $5.71 \pm 0.03$ & 2005 Feb 20 \\
\hline & & & $6.67 \pm 0.02$ & & $5.67 \pm 0.03$ & 2007 Oct 16 \\
\hline & IRAM 04191+1522 & $14.90 \pm 0.04$ & $13.28 \pm 0.04$ & $12.66 \pm 0.04$ & $12.49 \pm 0.05$ & 2004 Sep 10 \\
\hline & & $15.32 \pm 0.05$ & $13.81 \pm 0.03$ & $13.23 \pm 0.04$ & $12.90 \pm 0.04$ & 2005 Sep 17 \\
\hline J04215740+2826355 & RY Tau & sat & sat & $3.48 \pm 0.03$ & sat & 2005 Feb 20 \\
\hline & & sat & sat & & sat & 2005 Feb 21 \\
\hline J04215884+2818066 & HD 283572 & $6.82 \pm 0.02$ & $6.79 \pm 0.02$ & $6.76 \pm 0.03$ & $6.74 \pm 0.03$ & 2005 Feb 20 \\
\hline & & $6.83 \pm 0.02$ & $6.85 \pm 0.02$ & $6.76 \pm 0.03$ & $6.76 \pm 0.03$ & 2005 Feb 21 \\
\hline J042159 & $\mathrm{T}$ Tau N+S & sat & sat & $2.65 \pm 0.03$ & sat & 2005 Feb 20 \\
\hline J04220007+1530248 & IRAS $04191+1523 \mathrm{~B}$ & $12.18 \pm 0.03$ & $11.17 \pm 0.03$ & $10.68 \pm 0.04$ & $9.75 \pm 0.04$ & 2004 Sep 10 \\
\hline & & & & & $9.84 \pm 0.06$ & 2005 Sep 17 \\
\hline J04220043+1530212 & IRAS $04191+1523 \mathrm{~A}$ & $9.48 \pm 0.02$ & $8.30 \pm 0.02$ & $7.38 \pm 0.03$ & $6.41 \pm 0.03$ & 2004 Sep 10 \\
\hline & & $9.56 \pm 0.02$ & $8.29 \pm 0.02$ & $7.31 \pm 0.03$ & $6.26 \pm 0.03$ & 2005 Sep 17 \\
\hline J04220069+2657324 & Haro 6-5B & $9.47 \pm 0.02$ & $8.31 \pm 0.02$ & $7.23 \pm 0.03$ & $5.93 \pm 0.03$ & 2005 Feb 20 \\
\hline & & $9.63 \pm 0.02$ & $8.35 \pm 0.02$ & $7.16 \pm 0.03$ & $5.86 \pm 0.03$ & 2007 Oct 17 \\
\hline J04220217+2657304 & FS Tau A+B & $6.88 \pm 0.02$ & $6.35 \pm 0.02$ & $5.93 \pm 0.03$ & $5.14 \pm 0.03$ & 2005 Feb 20 \\
\hline & & $6.76 \pm 0.02$ & $6.37 \pm 0.02$ & $5.76 \pm 0.03$ & $4.89 \pm 0.03$ & 2007 Oct 17 \\
\hline J04220313+2825389 & $\mathrm{LkCa} 21$ & $8.23 \pm 0.02$ & $8.14 \pm 0.02$ & $8.12 \pm 0.03$ & $8.09 \pm 0.03$ & 2005 Feb 20 \\
\hline & & $8.22 \pm 0.02$ & $8.17 \pm 0.02$ & $8.13 \pm 0.03$ & $8.10 \pm 0.03$ & 2005 Feb 21 \\
\hline J04221332+1934392 & $\ldots$ & $11.02 \pm 0.02$ & $10.89 \pm 0.02$ & $10.82 \pm 0.03$ & $10.59 \pm 0.03$ & 2007 Mar 29 \\
\hline
\end{tabular}


TABLE $4-$ Continued

\begin{tabular}{|c|c|c|c|c|c|c|}
\hline $2 \mathrm{MASS}^{\mathrm{a}}$ & Name & {$[3.6]$} & {$[4.5]$} & {$[5.8]$} & {$[8.0]$} & Date \\
\hline J04221568+2657060 & XEST 11-078 & $11.05 \pm 0.02$ & $10.49 \pm 0.02$ & $10.20 \pm 0.03$ & $9.73 \pm 0.03$ & 2007 Oct 17 \\
\hline \multirow[t]{2}{*}{ J04221644+2549118 } & & $11.43 \pm 0.02$ & $11.33 \pm 0.02$ & $11.27 \pm 0.03$ & $11.26 \pm 0.03$ & 2006 Sep 28 \\
\hline & & $11.45 \pm 0.02$ & $11.26 \pm 0.02$ & $11.21 \pm 0.03$ & $11.22 \pm 0.03$ & 2007 Mar 30 \\
\hline J04221675+2654570 & & $7.71 \pm 0.02$ & $7.23 \pm 0.02$ & $6.79 \pm 0.03$ & $6.18 \pm 0.03$ & 2007 Oct 17 \\
\hline J04222404+2646258 & XEST 11-087 & $9.45 \pm 0.02$ & $9.36 \pm 0.02$ & $9.30 \pm 0.03$ & $9.33 \pm 0.03$ & 2007 Oct 17 \\
\hline \multirow{2}{*}{ J04224786+2645530 } & IRAS $04196+2638$ & $8.06 \pm 0.02$ & $7.49 \pm 0.02$ & $7.09 \pm 0.03$ & $6.24 \pm 0.03$ & 2005 Feb 21 \\
\hline & & $8.11 \pm 0.02$ & $7.55 \pm 0.02$ & $7.02 \pm 0.03$ & $6.27 \pm 0.03$ & 2007 Oct 17 \\
\hline \multirow{2}{*}{ J04230607+2801194 } & $\cdots$ & $10.60 \pm 0.02$ & out & $9.98 \pm 0.03$ & out & 2005 Feb 20 \\
\hline & & $10.58 \pm 0.02$ & $10.22 \pm 0.02$ & $9.93 \pm 0.03$ & $9.35 \pm 0.03$ & 2005 Feb 21 \\
\hline \multirow[t]{2}{*}{ J04230776+2805573 } & IRAS $04200+2759$ & $8.44 \pm 0.02$ & $7.86 \pm 0.02$ & $7.36 \pm 0.03$ & $6.49 \pm 0.03$ & 2005 Feb 20 \\
\hline & & $8.42 \pm 0.02$ & $7.74 \pm 0.02$ & $7.30 \pm 0.03$ & $6.41 \pm 0.03$ & 2005 Feb 21 \\
\hline J04231822+2641156 & & $9.39 \pm 0.02$ & $9.15 \pm 0.02$ & $8.76 \pm 0.03$ & $8.12 \pm 0.03$ & 2005 Feb 21 \\
\hline \multirow{2}{*}{ J04233539+2503026 } & FU Tau A & out & $7.68 \pm 0.02$ & out & $6.44 \pm 0.03$ & 2005 Feb 23 \\
\hline & & $8.34 \pm 0.02$ & $7.87 \pm 0.02$ & $7.33 \pm 0.03$ & $6.71 \pm 0.03$ & 2007 Mar 30 \\
\hline \multirow[t]{2}{*}{ J04233573+2502596 } & FU Tau B & out & $11.90 \pm 0.10$ & out & $10.77 \pm 0.10$ & 2005 Feb 23 \\
\hline & & $12.54 \pm 0.10$ & $11.93 \pm 0.10$ & $11.46 \pm 0.10$ & $10.87 \pm 0.10$ & 2007 Mar 30 \\
\hline \multirow[t]{2}{*}{ J04233919+2456141 } & FT Tau & $7.64 \pm 0.02$ & $7.12 \pm 0.02$ & $6.81 \pm 0.03$ & $5.95 \pm 0.03$ & 2005 Feb 23 \\
\hline & & $7.89 \pm 0.02$ & $7.44 \pm 0.02$ & $7.12 \pm 0.03$ & $6.27 \pm 0.03$ & 2007 Mar 30 \\
\hline J04242090+2630511 & & $11.80 \pm 0.02$ & $11.41 \pm 0.02$ & $11.00 \pm 0.03$ & $10.37 \pm 0.03$ & 2005 Feb 21 \\
\hline $\mathrm{J} 04242646+2649503$ & & $11.11 \pm 0.02$ & $10.80 \pm 0.02$ & $10.48 \pm 0.03$ & $9.85 \pm 0.03$ & 2005 Feb 21 \\
\hline J04244457+2610141 & IRAS $04216+2603$ & $7.80 \pm 0.02$ & $7.24 \pm 0.02$ & $6.78 \pm 0.03$ & $6.00 \pm 0.03$ & 2004 Mar 7 \\
\hline & & $8.07 \pm 0.02$ & $7.56 \pm 0.02$ & $7.07 \pm 0.03$ & $6.25 \pm 0.03$ & 2005 Feb 21 \\
\hline J04244506+2701447 & $\mathrm{J} 1-4423$ & $10.18 \pm 0.02$ & $10.11 \pm 0.02$ & $10.08 \pm 0.03$ & $10.04 \pm 0.03$ & 2005 Feb 20 \\
\hline & & $10.18 \pm 0.02$ & $10.14 \pm 0.02$ & $10.02 \pm 0.03$ & $10.06 \pm 0.03$ & 2005 Feb 21 \\
\hline J04245708+2711565 & IP Tau & $7.70 \pm 0.02$ & $7.43 \pm 0.02$ & $7.20 \pm 0.03$ & $6.53 \pm 0.03$ & 2005 Feb 20 \\
\hline & & $7.76 \pm 0.02$ & $7.42 \pm 0.02$ & $7.22 \pm 0.03$ & $6.60 \pm 0.03$ & 2005 Feb 21 \\
\hline & & $7.67 \pm 0.02$ & $7.38 \pm 0.02$ & $7.20 \pm 0.03$ & $6.61 \pm 0.03$ & 2007 Oct 16 \\
\hline J04251767+2617504 & J1-4872 B & $9.06 \pm 0.04$ & $8.94 \pm 0.04$ & $8.88 \pm 0.04$ & $8.86 \pm 0.04$ & 2004 Mar 7 \\
\hline & & $9.02 \pm 0.04$ & $8.95 \pm 0.04$ & $8.82 \pm 0.04$ & $8.88 \pm 0.04$ & 2005 Feb 21 \\
\hline J04251767+2617504 & J1-4872 A & $8.33 \pm 0.04$ & $8.32 \pm 0.04$ & $8.25 \pm 0.04$ & $8.22 \pm 0.04$ & 2004 Mar 7 \\
\hline & & $8.32 \pm 0.04$ & $8.24 \pm 0.04$ & $8.30 \pm 0.04$ & $8.18 \pm 0.04$ & 2005 Feb 21 \\
\hline J04262939+2624137 & KPNO 3 & $11.36 \pm 0.02$ & $10.92 \pm 0.02$ & $10.51 \pm 0.03$ & $9.66 \pm 0.03$ & 2004 Mar 7 \\
\hline & & $11.39 \pm 0.02$ & $10.97 \pm 0.02$ & $10.45 \pm 0.03$ & $9.70 \pm 0.03$ & 2005 Feb 22 \\
\hline J04263055+2443558 & $\cdots$ & $12.58 \pm 0.02$ & $12.20 \pm 0.02$ & $11.79 \pm 0.03$ & $11.06 \pm 0.03$ & 2007 Mar 28 \\
\hline & & $12.56 \pm 0.02$ & $12.15 \pm 0.02$ & $11.76 \pm 0.03$ & $11.06 \pm 0.03$ & 2007 Oct 16 \\
\hline J04265352+2606543 & FV Tau A+B & $6.12 \pm 0.02$ & $5.49 \pm 0.02$ & $4.88 \pm 0.03$ & $4.08 \pm 0.03$ & 2004 Mar 7 \\
\hline & & $6.32 \pm 0.02$ & $5.79 \pm 0.02$ & $5.20 \pm 0.03$ & $4.42 \pm 0.03$ & 2005 Feb 22 \\
\hline J04265440+2606510 & FV Tau/c A+B & $8.09 \pm 0.02$ & $7.66 \pm 0.02$ & $7.10 \pm 0.03$ & $6.30 \pm 0.03$ & 2004 Mar 7 \\
\hline & & $8.03 \pm 0.02$ & $7.51 \pm 0.02$ & $6.99 \pm 0.03$ & $6.28 \pm 0.03$ & 2005 Feb 22 \\
\hline J04265629+2443353 & IRAS $04239+2436$ & $7.62 \pm 0.02$ & $6.25 \pm 0.02$ & $5.29 \pm 0.03$ & $4.45 \pm 0.03$ & 2005 Feb 20 \\
\hline & & $7.57 \pm 0.02$ & $6.27 \pm 0.02$ & $5.34 \pm 0.03$ & $4.49 \pm 0.03$ & 2005 Feb 23 \\
\hline J04265732+2606284 & KPNO 13 & $8.62 \pm 0.02$ & $8.22 \pm 0.02$ & \pm 0.03 & $7.04 \pm 0.03$ & 2004 Mar 7 \\
\hline & & $8.75 \pm 0.02$ & $8.32 \pm 0.02$ & $7.91 \pm 0.03$ & $7.37 \pm 0.03$ & 2005 Feb 22 \\
\hline J04270266+2605304 & DG Tau B & $8.86 \pm 0.02$ & $7.21 \pm 0.02$ & $5.85 \pm 0.03$ & $4.83 \pm 0.03$ & 2004 Mar 7 \\
\hline & & $8.78 \pm 0.02$ & $7.07 \pm 0.02$ & $5.72 \pm 0.03$ & $4.62 \pm 0.03$ & 2005 Feb 22 \\
\hline J04270280+2542223 & DF Tau A+B & $6.03 \pm 0.02$ & $5.60 \pm 0.02$ & $5.21 \pm 0.03$ & $4.61 \pm 0.03$ & 2004 Mar 7 \\
\hline & & sat & $5.36 \pm 0.02$ & $4.99 \pm 0.03$ & $4.42 \pm 0.03$ & 2005 Feb 20 \\
\hline & & sat & out & $4.99 \pm 0.03$ & out & 2005 Feb 22 \\
\hline J04270469+2606163 & DG Tau & sat & sat & $=0.03$ & sat & 2004 Mar 7 \\
\hline & & sat & $5.05 \pm 0.02$ & $=0.03$ & $3.57 \pm 0.03$ & 2005 Feb 22 \\
\hline J04270739+2215037 & & $10.90 \pm 0.02$ & $10.78 \pm 0.02$ & $10.74 \pm 0.03$ & $10.73 \pm 0.03$ & 2008 Oct 31 \\
\hline J04272799+2612052 & KPNO 4 & $12.56 \pm 0.02$ & $12.35 \pm 0.02$ & $12.23 \pm 0.04$ & $12.11 \pm 0.04$ & 2004 Mar 7 \\
\hline & & $12.54 \pm 0.02$ & $12.32 \pm 0.02$ & $12.23 \pm 0.04$ & $12.13 \pm 0.05$ & 2005 Feb 22 \\
\hline $8+2357243$ & & $13.20 \pm 0.02$ & $13.05 \pm 0.02$ & $12.98 \pm 0.04$ & $13.00 \pm 0.07$ & 2005 Feb 20 \\
\hline J0427573 & IRAS $04248+2612$ & $9.54 \pm 0.03$ & $8.69 \pm 0.02$ & $7.88 \pm 0.03$ & $6.85 \pm 0.03$ & 2004 Mar 7 \\
\hline & & $9.78 \pm 0.03$ & $9.02 \pm 0.02$ & $8.26 \pm 0.03$ & $7.11 \pm 0.03$ & 2005 Feb 22 \\
\hline$\cdots$ & L1521F-IRS & & 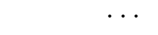 & & $12.47 \pm 0.04$ & 2004 Sep 9 \\
\hline & & & $\cdots$ & $\cdots$ & $12.55 \pm 0.06$ & 2005 Feb 24 \\
\hline & & & & & $12.37 \pm 0.03$ & 2006 Mar 25 \\
\hline J04284263+2714039 & $\cdots$ & $9.69 \pm 0.02$ & $9.45 \pm 0.02$ & $9.19 \pm 0.03$ & $8.83 \pm 0$ & 2005 Feb 24 \\
\hline & & $12.25 \pm 0.02$ & $11.95 \pm 0.02$ & \pm 0.03 & $10.96 \pm 0.03$ & 2005 Feb 24 \\
\hline J04290498+2649073 & IRAS $04260+2642$ & $10.01 \pm 0.02$ & $9.37 \pm 0.02$ & $8.82 \pm 0.03$ & $8.03 \pm 0.03$ & 2004 Mar 7 \\
\hline & & $10.03 \pm 0.02$ & $9.34 \pm 0.02$ & $8.86 \pm 0.03$ & $8.08 \pm 0.03$ & 2005 Feb 24 \\
\hline J04292071+2633406 & $\mathrm{J} 1-507$ & $8.54 \pm 0.02$ & $8.47 \pm 0.02$ & $8.44 \pm 0.03$ & $8.40 \pm 0.03$ & 2004 Mar 7 \\
\hline & & $8.51 \pm 0.02$ & $8.52 \pm 0.02$ & $8.45 \pm 0.03$ & $8.48 \pm 0.03$ & 2005 Feb 24 \\
\hline J04292165+2701259 & IRAS $04263+2654$ & $8.07 \pm 0.02$ & $7.64 \pm 0.02$ & $7.27 \pm 0.03$ & $6.64 \pm 0.03$ & 2005 Feb 24 \\
\hline & GV Tau A+B & sat & sat & $2.55 \pm 0.03$ & sat & 2005 Feb 23 \\
\hline & & sat & sat & $2.57 \pm 0.03$ & sat & 2005 Feb 24 \\
\hline & & sat & sat & $2.64 \pm 0.03$ & sat & 2007 Oct 16 \\
\hline J04292971+2616532 & $\mathrm{FW}$ Tau $\mathrm{A}+\mathrm{B}+\mathrm{C}$ & $9.03 \pm 0.02$ & $8.92 \pm 0.02$ & $8.89 \pm 0.03$ & $8.86 \pm 0.03$ & 2004 Mar 7 \\
\hline & & & $8.93 \pm 0.02$ & $8.90 \pm 0.03$ & $8.88 \pm 0.03$ & 2005 Feb 24 \\
\hline & & $9.07 \pm 0.02$ & $8.90 \pm 0.02$ & $8.90 \pm 0.03$ & $8.87 \pm 0.03$ & 2006 Mar 25 \\
\hline J04293008+2439550 & IRAS $04264+2433$ & $10.19 \pm 0.02$ & $9.48 \pm 0.02$ & $8.79 \pm 0.03$ & $6.97 \pm 0.03$ & 2004 Sep 7 \\
\hline & & out & $9.13 \pm 0.02$ & out & $6.44 \pm 0.03$ & 2005 Feb 23 \\
\hline & & $10.16 \pm 0.02$ & $9.40 \pm 0.02$ & $8.55 \pm 0.03$ & $6.70 \pm 0.03$ & 2005 Feb 24 \\
\hline J04293209+2430597 & & out & $9.20 \pm 0.02$ & out & $7.26 \pm 0.03$ & 2004 Sep 7 \\
\hline & & $10.42 \pm 0.02$ & $9.20 \pm 0.02$ & $8.24 \pm 0.03$ & $7.35 \pm 0.03$ & 2005 Feb 23 \\
\hline
\end{tabular}


TABLE $4-$ Continued

\begin{tabular}{|c|c|c|c|c|c|c|}
\hline $2 \mathrm{MASS}^{\mathrm{a}}$ & Name & {$[3.6]$} & {$[4.5]$} & {$[5.8]$} & {$[8.0]$} & Date \\
\hline \multirow{3}{*}{ J04293606+2435556 } & \multirow{3}{*}{ XEST 13-010 } & $10.36 \pm 0.02$ & $9.22 \pm 0.02$ & $8.29 \pm 0.03$ & $7.31 \pm 0.03$ & 2005 Feb 24 \\
\hline & & out & $7.73 \pm 0.02$ & out & $7.04 \pm 0.03$ & 2004 Sep 7 \\
\hline & & $7.99 \pm 0.02$ & $7.71 \pm 0.02$ & $7.42 \pm 0.03$ & $6.96 \pm 0.03$ & 2005 Feb 24 \\
\hline \multirow[t]{2}{*}{ J04294155+2632582 } & \multirow[t]{2}{*}{$\mathrm{DH}$ Tau A+B } & $7.47 \pm 0.02$ & $7.19 \pm 0.02$ & $7.11 \pm 0.03$ & $6.74 \pm 0.03$ & 2004 Mar 7 \\
\hline & & $7.60 \pm 0.02$ & $7.41 \pm 0.02$ & $7.19 \pm 0.03$ & $6.81 \pm 0.03$ & 2005 Feb 24 \\
\hline \multirow[t]{2}{*}{ J04294247+2632493 } & \multirow[t]{2}{*}{ DI Tau A+B } & $8.16 \pm 0.02$ & $8.25 \pm 0.02$ & $8.16 \pm 0.03$ & $8.11 \pm 0.03$ & 2004 Mar 7 \\
\hline & & $8.22 \pm 0.02$ & $8.20 \pm 0.02$ & $8.11 \pm 0.03$ & $8.10 \pm 0.03$ & 2005 Feb 24 \\
\hline \multirow{2}{*}{ J04294568+2630468 } & KPNO 5 & $11.05 \pm 0.02$ & $10.92 \pm 0.02$ & $10.81 \pm 0.03$ & $10.85 \pm 0.03$ & 2004 Mar 7 \\
\hline & & $11.04 \pm 0.02$ & $10.93 \pm 0.02$ & $10.89 \pm 0.03$ & $10.84 \pm 0.03$ & 2005 Feb 24 \\
\hline J04295156+2606448 & IQ Tau & $6.94 \pm 0.02$ & $6.49 \pm 0.02$ & $6.12 \pm 0.03$ & $5.57 \pm 0.03$ & 2004 Mar 7 \\
\hline & & $6.73 \pm 0.02$ & $6.32 \pm 0.02$ & $6.02 \pm 0.03$ & $5.46 \pm 0.03$ & 2005 Feb 24 \\
\hline J04295422+1754041 & $\ldots$ & out & out & out & out & \\
\hline J04295950+2433078 & $\cdots$ & sat & out & $8.28 \pm 0.03$ & out & 2004 Oct 7 \\
\hline & & $9.03 \pm 0.02$ & $8.56 \pm 0.02$ & $8.31 \pm 0.03$ & $7.80 \pm 0.03$ & 2005 Feb 24 \\
\hline J04300357+1813494 & UX Tau B & $8.75 \pm 0.04$ & $8.75 \pm 0.04$ & $8.69 \pm 0.04$ & $8.66 \pm 0.04$ & 2005 Feb 19 \\
\hline J04300399+1813493 & UX Tau A+C & $6.66 \pm 0.02$ & $6.37 \pm 0.02$ & $6.16 \pm 0.03$ & $5.93 \pm 0.03$ & 2005 Feb 19 \\
\hline J04300724+2608207 & KPNO 6 & $13.08 \pm 0.02$ & $12.77 \pm 0.02$ & $12.41 \pm 0.04$ & $11.82 \pm 0.04$ & 2004 Mar 7 \\
\hline & & $13.09 \pm 0.02$ & $12.73 \pm 0.02$ & $12.41 \pm 0.04$ & $11.54 \pm 0.04$ & 2005 Feb 24 \\
\hline J04302365+2359129 & & $13.21 \pm 0.02$ & $13.11 \pm 0.02$ & $13.03 \pm 0.05$ & $13.02 \pm 0.06$ & 2005 Feb 24 \\
\hline J04302961+2426450 & FX Tau A+B & $7.21 \pm 0.02$ & $6.92 \pm 0.02$ & $6.62 \pm 0.03$ & $5.90 \pm 0.03$ & 2005 Feb 20 \\
\hline & & $7.17 \pm 0.02$ & $6.87 \pm 0.02$ & $6.66 \pm 0.03$ & $5.94 \pm 0.03$ & 2005 Feb 24 \\
\hline J04304425+2601244 & DK Tau A & $5.88 \pm 0.02$ & $5.49 \pm 0.02$ & $5.24 \pm 0.03$ & $4.58 \pm 0.03$ & 2004 Mar 7 \\
\hline & & $6.12 \pm 0.02$ & $5.71 \pm 0.02$ & $5.39 \pm 0.03$ & $4.66 \pm 0.03$ & 2005 Feb 24 \\
\hline J04304425+2601244 & DK Tau B & $8.02 \pm 0.10$ & $7.30 \pm 0.10$ & $7.24 \pm 0.10$ & $6.60 \pm 0.10$ & 2004 Mar 7 \\
\hline & & $7.77 \pm 0.10$ & $7.41 \pm 0.10$ & $7.39 \pm 0.10$ & $6.63 \pm 0.10$ & 2005 Feb 24 \\
\hline J04305028+2300088 & IRAS $04278+2253 \mathrm{~A}+\mathrm{B}$ & sat & sat & $3.21 \pm 0.03$ & sat & 2005 Feb 20 \\
\hline & & sat & sat & $3.33 \pm 0.03$ & sat & 2005 Feb 24 \\
\hline J04305137+2442222 & ZZ Tau & $8.10 \pm 0.02$ & $7.87 \pm 0.02$ & $7.59 \pm 0.03$ & $6.96 \pm 0.03$ & 2005 Feb 23 \\
\hline & & $8.05 \pm 0.02$ & $7.87 \pm 0.02$ & $7.64 \pm 0.03$ & $6.99 \pm 0.03$ & 2005 Feb 24 \\
\hline J04305171+2441475 & ZZ Tau IRS & $8.15 \pm 0.02$ & $7.35 \pm 0.02$ & $6.67 \pm 0.03$ & $5.75 \pm 0.03$ & 2005 Feb 23 \\
\hline & & $8.06 \pm 0.02$ & $7.32 \pm 0.02$ & $6.66 \pm 0.03$ & $5.72 \pm 0.03$ & 2005 Feb 24 \\
\hline J04305718+2556394 & KPNO 7 & $12.60 \pm 0.02$ & $12.27 \pm 0.02$ & $11.93 \pm 0.03$ & $11.26 \pm 0.03$ & 2004 Mar 7 \\
\hline & & $12.58 \pm 0.02$ & $12.22 \pm 0.02$ & $12.00 \pm 0.04$ & $11.25 \pm 0.03$ & 2005 Feb 24 \\
\hline J04311444+2710179 & JH 56 & $8.77 \pm 0.02$ & $8.73 \pm 0.02$ & $8.69 \pm 0.03$ & $8.64 \pm 0.03$ & 2004 Mar 7 \\
\hline & & $8.70 \pm 0.02$ & $8.72 \pm 0.02$ & $8.70 \pm 0.03$ & $8.60 \pm 0.03$ & 2005 Feb 24 \\
\hline J04311578+1820072 & MHO 9 & $10.04 \pm 0.02$ & $9.96 \pm 0.02$ & $9.86 \pm 0.03$ & $9.94 \pm 0.03$ & 2004 Oct 7 \\
\hline & & out & $9.93 \pm 0.02$ & out & $9.88 \pm 0.03$ & 2006 Mar 23 \\
\hline J04311907+23 & & $11.65 \pm 0.02$ & $11.52 \pm 0.02$ & $11.53 \pm 0.03$ & $11.47 \pm 0.04$ & 2005 Feb 24 \\
\hline J04312382 & V927 Tau A+B & $8.52 \pm 0.02$ & $8.45 \pm 0.02$ & $8.41 \pm 0.03$ & $8.39 \pm 0.03$ & 2005 Feb 20 \\
\hline & & $8.52 \pm 0.02$ & $8.40 \pm 0.02$ & $8.37 \pm 0.03$ & $8.38 \pm 0.03$ & 2005 Feb 24 \\
\hline J04312405+1800215 & MHO 4 & $10.12 \pm 0.02$ & $9.99 \pm 0.02$ & $9.94 \pm 0.03$ & $9.93 \pm 0.03$ & 2004 Oct 7 \\
\hline & & $12.85 \pm 0.02$ & out & $12.73 \pm 0.04$ & out & 2004 Mar 7 \\
\hline & & $12.88 \pm 0.02$ & $12.69 \pm 0.02$ & $12.68 \pm 0.04$ & $12.68 \pm 0.04$ & 2005 Feb 24 \\
\hline J04313407+1808049 & L1551/IRS5 & $7.03 \pm 0.02$ & $5.54 \pm 0.02$ & $4.16 \pm 0.03$ & $2.78 \pm 0.03$ & 2004 Oct 7 \\
\hline & LkHa 358 & $8.45 \pm 0.02$ & $7.72 \pm 0.02$ & $7.12 \pm 0.03$ & $6.43 \pm 0.03$ & 2004 Oct 7 \\
\hline J04 & HH 30 & $12.38 \pm 0.02$ & $11.81 \pm 0.02$ & $11.64 \pm 0.03$ & $11.25 \pm 0$ & 2004 Oct 7 \\
\hline J04313843+1813576 & HL Tau & sat & sat & $3.57 \pm 0.03$ & $2.93 \pm 0.03$ & 2004 Oct 7 \\
\hline J04314007+1813571 & XZ Tau A+B & sat & $5.04 \pm 0.02$ & $4.40 \pm 0.03$ & $3.57 \pm 0.03$ & 2004 Oct 7 \\
\hline J04314444+1808315 & L1551NE & $8.66 \pm 0.02$ & $7.06 \pm 0.02$ & $5.98 \pm 0.03$ & $5.03 \pm 0.03$ & 2004 Oct 7 \\
\hline J04315056+2424180 & HK Tau A+B & out & sat & out & $6.61 \pm 0.03$ & 2004 Oct 7 \\
\hline & & $7.78 \pm 0.02$ & $7.39 \pm 0.02$ & $7.08 \pm 0.03$ & & 2005 Feb 20 \\
\hline & & $7.68 \pm 0.02$ & $7.30 \pm$ & $7.06 \pm 0.03$ & $6.55 \pm 0.03$ & 2005 Feb 24 \\
\hline J04315779+1821380 & V710 Tau A & $7.95 \pm 0.04$ & $7.55 \pm 0.04$ & $7.16 \pm 0.04$ & $6.36 \pm 0.04$ & 2004 Oct 7 \\
\hline & V710 T & $8.34 \pm 0.04$ & $8.13 \pm 0.05$ & $8.11 \pm 0.07$ & $7.91 \pm 0.10$ & 2004 Oct 7 \\
\hline J04315844+2543299 & $\mathrm{J} 1-665$ & $9.33 \pm 0.02$ & $9.25 \pm 0.02$ & $9.22 \pm 0.03$ & $9.21 \pm 0.03$ & 2004 Mar 7 \\
\hline & & $9.29 \pm 0.02$ & $9.24 \pm 0.02$ & $9.23 \pm 0.03$ & $9.27 \pm 0.03$ & 2005 Feb 24 \\
\hline J04315968+1821305 & LkHa 267 & $8.78 \pm 0.02$ & $8.24 \pm 0.02$ & $7.82 \pm 0.03$ & $7.31 \pm 0.03$ & 2004 Oct 7 \\
\hline & & $10.27 \pm 0.02$ & $10.14 \pm 0.02$ & $10.09 \pm 0.03$ & $10.09 \pm 0.03$ & 2005 Feb 24 \\
\hline J04320926+1757227 & L1551-51 & out & $8.69 \pm 0.02$ & out & $8.64 \pm 0.03$ & 2004 Oct 7 \\
\hline & & $8.79 \pm 0.02$ & $8.72 \pm 0.02$ & $8.71 \pm 0.03$ & $8.67 \pm 0.03$ & 2005 Feb 19 \\
\hline J04321456+1820147 & V827 Tau & $8.12 \pm 0.02$ & $8.05 \pm 0.02$ & $8.01 \pm 0.03$ & $8.02 \pm 0.03$ & 2004 Oct 7 \\
\hline J04321540+2428597 & Haro $6-13$ & $6.23 \pm 0.02$ & $5.72 \pm 0.02$ & $5.34 \pm 0.03$ & $4.66 \pm 0.03$ & 2005 Feb 20 \\
\hline & & $6.30 \pm 0.02$ & $5.80 \pm 0.02$ & $5.39 \pm 0.03$ & $4.74 \pm 0.03$ & 2005 Feb 24 \\
\hline J04321583+1801387 & V826 Tau A+B & $8.04 \pm 0.02$ & $8.08 \pm 0.02$ & $8.03 \pm 0.03$ & $7.97 \pm 0.03$ & 2004 Oct 7 \\
\hline J04321606+1812464 & MHO 5 & $9.30 \pm 0.02$ & $8.95 \pm 0.02$ & $8.56 \pm 0.03$ & $7.85 \pm 0.03$ & 2004 Oct 7 \\
\hline J04321786+2422149 & & $9.91 \pm 0.02$ & $9.79 \pm 0.02$ & $9.74 \pm 0.03$ & $9.72 \pm 0.03$ & 2005 Feb 20 \\
\hline & & $9.96 \pm 0.02$ & $9.81 \pm 0.02$ & $9.73 \pm 0.03$ & $9.72 \pm 0.03$ & 2005 Feb 24 \\
\hline J04321885+2422271 & V928 Tau A+B & $7.88 \pm 0.02$ & $7.83 \pm 0.02$ & $7.74 \pm 0.03$ & $7.65 \pm 0.03$ & 2005 Feb 20 \\
\hline & & $7.80 \pm 0.02$ & $7.75 \pm 0.02$ & $7.70 \pm 0.03$ & $7.64 \pm 0.03$ & 2005 Feb 24 \\
\hline J04322210+1827426 & MHO 6 & $10.10 \pm 0.02$ & out & $9.61 \pm 0.03$ & out & 2004 Oct 7 \\
\hline & & $10.01 \pm 0.02$ & $9.74 \pm 0.02$ & $9.53 \pm 0.03$ & $9.06 \pm 0.03$ & 2005 Feb 19 \\
\hline $\mathrm{J} 04322329+24$ & $\ldots$ & $10.92 \pm 0.02$ & $10.78 \pm 0.02$ & $10.84 \pm 0.03$ & $10.69 \pm 0.03$ & 2005 Feb 24 \\
\hline J04322415+2251083 & & $9.97 \pm 0.02$ & out & $9.26 \pm 0.03$ & out & 2005 Feb 24 \\
\hline & & $10.03 \pm 0.02$ & $9.64 \pm 0.02$ & $9.36 \pm 0.03$ & $8.47 \pm 0.03$ & 2007 Apr 3 \\
\hline J04322627+1827521 & MHO 7 & $9.87 \pm 0.02$ & out & $9.78 \pm 0.03$ & out & 2004 Oct 7 \\
\hline & & $9.87 \pm 0.02$ & $9.79 \pm 0.02$ & $9.74 \pm 0.03$ & $9.74 \pm 0.03$ & 2005 Feb 19 \\
\hline J04323028+1731303 & GG Tau $\mathrm{Ba}+\mathrm{Bb}$ & $9.20 \pm 0.02$ & $8.80 \pm 0.02$ & $8.40 \pm 0.03$ & $7.61 \pm 0.03$ & 2005 Feb 19 \\
\hline
\end{tabular}


TABLE 4 - Continued

\begin{tabular}{|c|c|c|c|c|c|c|}
\hline $2 \mathrm{MASS}^{\mathrm{a}}$ & Name & {$[3.6]$} & {$[4.5]$} & {$[5.8]$} & {$[8.0]$} & Date \\
\hline J04323034+1731406 & GG Tau $\mathrm{Aa}+\mathrm{Ab}$ & $6.62 \pm 0.02$ & $6.26 \pm 0.02$ & $5.90 \pm 0.03$ & $5.03 \pm 0.03$ & 2005 Feb 19 \\
\hline \multirow{3}{*}{ J04323058+2419572 } & FY Tau & sat & sat & $6.46 \pm 0.03$ & sat & 2004 Oct 7 \\
\hline & & $7.20 \pm 0.02$ & $6.82 \pm 0.02$ & $6.50 \pm 0.03$ & $6.02 \pm 0.03$ & 2005 Feb 20 \\
\hline & & $7.12 \pm 0.02$ & $6.72 \pm 0.02$ & $6.45 \pm 0.03$ & $5.97 \pm 0.03$ & 2005 Feb 24 \\
\hline \multirow[t]{2}{*}{ J04323176+2420029 } & FZ Tau & $6.30 \pm 0.02$ & $5.66 \pm 0.02$ & $5.23 \pm 0.03$ & $4.48 \pm 0.03$ & 2005 Feb 20 \\
\hline & & $6.28 \pm 0.02$ & $5.73 \pm 0.02$ & $5.24 \pm 0.03$ & $4.50 \pm 0.03$ & 2005 Feb 24 \\
\hline \multirow[t]{3}{*}{ J04323205+2257266 } & IRAS $04295+2251$ & $8.60 \pm 0.02$ & $7.70 \pm 0.02$ & $6.78 \pm 0.03$ & $5.49 \pm 0.03$ & 2005 Feb 20 \\
\hline & & $8.56 \pm 0.02$ & $7.70 \pm 0.02$ & $6.81 \pm 0.03$ & $5.50 \pm 0.03$ & 2005 Feb 24 \\
\hline & & out & $7.62 \pm 0.02$ & out & $5.06 \pm 0.03$ & 2007 Apr 3 \\
\hline \multirow[t]{2}{*}{ J04324282+2552314 } & UZ Tau Ba+Bb & $7.68 \pm 0.10$ & $7.55 \pm 0.10$ & $7.17 \pm 0.10$ & $6.50 \pm 0.10$ & 2004 Mar 7 \\
\hline & & $7.71 \pm 0.07$ & $7.38 \pm 0.07$ & $7.12 \pm 0.07$ & $6.41 \pm 0.07$ & 2005 Feb 24 \\
\hline \multirow{2}{*}{ J04324303+2552311 } & UZ Tau A & $5.91 \pm 0.02$ & $5.39 \pm 0.02$ & $5.00 \pm 0.03$ & $4.20 \pm 0.03$ & 2004 Mar 7 \\
\hline & & $6.41 \pm 0.02$ & $5.87 \pm 0.02$ & $5.45 \pm 0.03$ & $4.59 \pm 0.03$ & 2005 Feb 24 \\
\hline J04324373+1802563 & L1551-55 & $9.19 \pm 0.02$ & $9.16 \pm 0.02$ & $9.12 \pm 0.03$ & $9.10 \pm 0.03$ & 2005 Feb 19 \\
\hline \multirow[t]{3}{*}{$\mathrm{J} 04324911+2253027$} & JH 112 & $7.42 \pm 0.02$ & $7.11 \pm 0.02$ & $6.90 \pm 0.04$ & $5.91 \pm 0.04$ & 2005 Feb 20 \\
\hline & & $7.39 \pm 0.02$ & out & $6.88 \pm 0.04$ & out & 2005 Feb 24 \\
\hline & & $7.39 \pm 0.02$ & $7.13 \pm 0.03$ & $6.74 \pm 0.04$ & $5.82 \pm 0.03$ & 2007 Apr 3 \\
\hline \multirow{3}{*}{ J04324938+2253082 } & $\cdots$ & $8.45 \pm 0.02$ & $8.19 \pm 0.03$ & $7.74 \pm 0.04$ & $7.00 \pm 0.04$ & 2005 Feb 20 \\
\hline & & $8.49 \pm 0.02$ & out & $7.80 \pm 0.05$ & out & 2005 Feb 24 \\
\hline & & $8.52 \pm 0.02$ & $8.17 \pm 0.02$ & $7.76 \pm 0.04$ & $7.08 \pm 0.04$ & 2007 Apr 3 \\
\hline J04325026+2422115 & $\cdots$ & $10.42 \pm 0.02$ & $10.19 \pm 0.02$ & $10.08 \pm 0.03$ & $10.05 \pm 0.03$ & 2004 Oct 7 \\
\hline & & $10.44 \pm 0.02$ & $10.18 \pm 0.02$ & $10.09 \pm 0.03$ & $10.01 \pm 0.03$ & 2005 Feb 20 \\
\hline & & $10.42 \pm 0.02$ & $10.22 \pm 0.02$ & $10.07 \pm 0.03$ & $10.05 \pm 0.03$ & 2005 Feb 24 \\
\hline J04325119+1730092 & LH $0429+17$ & out & $12.90 \pm 0.02$ & out & $12.75 \pm 0.08$ & 2004 Sep 7 \\
\hline $\mathrm{J} 04330197+2421000$ & MHO 8 & sat & $9.16 \pm 0.02$ & $9.13 \pm 0.03$ & $9.07 \pm 0.03$ & 2004 Oct 7 \\
\hline & & $9.30 \pm 0.02$ & $9.16 \pm 0.02$ & $9.12 \pm 0.03$ & $9.08 \pm 0.03$ & 2005 Feb 20 \\
\hline & & $9.26 \pm 0.02$ & $9.27 \pm 0.02$ & $9.09 \pm 0.03$ & $9.10 \pm 0.03$ & 2005 Feb 24 \\
\hline & & $9.30 \pm 0.02$ & out & $9.12 \pm 0.03$ & out & 2006 Mar 26 \\
\hline J04330622+2409339 & GH Tau A+B & $7.01 \pm 0.02$ & $6.67 \pm 0.02$ & $6.43 \pm 0.03$ & $5.92 \pm 0.03$ & 2005 Feb 20 \\
\hline & & $7.06 \pm 0.02$ & $6.70 \pm 0.02$ & $6.57 \pm 0.03$ & $5.99 \pm 0.03$ & 2005 Feb 24 \\
\hline J04330664+2409549 & V807 Tau A+B & $6.39 \pm 0.02$ & $6.13 \pm 0.02$ & $5.85 \pm 0.03$ & $5.41 \pm 0.03$ & 2005 Feb 20 \\
\hline & & $6.45 \pm 0.02$ & $6.15 \pm 0.02$ & $5.89 \pm 0.03$ & $5.50 \pm 0.03$ & 2005 Feb 24 \\
\hline J04330781+2616066 & KPNO 14 & $9.78 \pm 0.02$ & $9.60 \pm 0.02$ & $9.59 \pm 0.03$ & $9.53 \pm 0.03$ & 2004 Mar 7 \\
\hline & & $9.72 \pm 0.02$ & $9.64 \pm 0.02$ & $9.59 \pm 0.03$ & $9.58 \pm 0.03$ & 2005 Feb 24 \\
\hline J04330945+2246487 & $\cdots$ & $10.77 \pm 0.02$ & $10.54 \pm 0.02$ & $10.31 \pm 0.03$ & $9.93 \pm 0.03$ & 2005 Feb 20 \\
\hline & & $10.80 \pm 0.02$ & $10.53 \pm 0.02$ & $10.27 \pm 0.03$ & $9.80 \pm 0.03$ & 2007 Mar 29 \\
\hline & & $10.78 \pm 0.02$ & $10.56 \pm 0.02$ & $10.32 \pm 0.03$ & $9.95 \pm 0.03$ & 2007 Apr 3 \\
\hline $\mathrm{J} 04331003+2433433$ & V830 Tau & $8.39 \pm 0.02$ & $8.37 \pm 0.02$ & $8.32 \pm 0.03$ & $8.30 \pm 0.03$ & 2005 Feb 20 \\
\hline & & $8.34 \pm 0.02$ & $8.40 \pm 0.02$ & $8.32 \pm 0.03$ & $8.31 \pm 0.03$ & 2005 Feb 24 \\
\hline J04331435+2614235 & IRAS $04301+2608$ & $11.84 \pm 0.02$ & $11.44 \pm 0.02$ & $11.02 \pm 0.03$ & $9.45 \pm 0.03$ & 2004 Mar 7 \\
\hline & & $12.04 \pm 0.02$ & $11.67 \pm 0.02$ & $11.27 \pm 0.03$ & $9.55 \pm 0.03$ & 2005 Feb 24 \\
\hline J04331650+2253204 & IRAS $04302+2247$ & $10.09 \pm 0.03$ & $9.68 \pm 0.02$ & $9.53 \pm 0.03$ & $9.62 \pm 0.03$ & 2005 Feb 20 \\
\hline & & $10.11 \pm 0.02$ & out & $9.55 \pm 0.03$ & out & 2005 Feb 24 \\
\hline & & out & $9.97 \pm 0.03$ & out & $9.66 \pm 0.03$ & 2007 Mar 29 \\
\hline & & $10.23 \pm 0.02$ & $9.85 \pm 0.03$ & $9.61 \pm 0.03$ & $9.63 \pm 0.03$ & 2007 Apr 3 \\
\hline $\mathrm{J} 04331907+2246342$ & IRAS $04303+2240$ & $6.16 \pm 0.02$ & $5.36 \pm 0.02$ & $4.68 \pm 0.03$ & $3.96 \pm 0.03$ & 2005 Feb 20 \\
\hline & & sat & sat & $4.33 \pm 0.03$ & $3.67 \pm 0.03$ & 2007 Apr 3 \\
\hline $\mathrm{J} 04332621+2245293$ & XEST 17-036 & $9.53 \pm 0.02$ & $9.44 \pm 0.02$ & $9.31 \pm 0.03$ & $9.35 \pm 0.03$ & 2005 Feb 20 \\
\hline & & $9.54 \pm 0.02$ & $9.39 \pm 0.02$ & $9.32 \pm 0.03$ & $9.31 \pm 0.03$ & 2007 Apr 3 \\
\hline J04333278+1800436 & $\cdots$ & out & $8.71 \pm 0.02$ & out & $7.33 \pm 0.03$ & 2005 Feb 19 \\
\hline J04333297+1801004 & HD 28867 B & out & $6.72 \pm 0.04$ & out & $6.19 \pm 0.04$ & 2005 Feb 19 \\
\hline J04333297+1801004 & HD $28867 \mathrm{~A}+\mathrm{C}$ & out & $6.15 \pm 0.04$ & out & $6.13 \pm 0.04$ & 2005 Feb 19 \\
\hline $\mathrm{J} 04333405+2421170$ & GI Tau & $6.80 \pm 0.02$ & $6.23 \pm 0.02$ & $5.72 \pm 0.03$ & $4.76 \pm 0.03$ & 2005 Feb 20 \\
\hline & & $6.80 \pm 0.02$ & $6.22 \pm 0.02$ & $5.74 \pm 0.03$ & $4.78 \pm 0.03$ & 2005 Feb 24 \\
\hline J04333456+2421058 & GK Tau & $6.43 \pm 0.02$ & $6.05 \pm 0.02$ & $5.77 \pm 0.03$ & $4.84 \pm 0.03$ & 2005 Feb 20 \\
\hline & & $6.43 \pm 0.02$ & $6.05 \pm 0.02$ & $5.72 \pm 0.03$ & $4.81 \pm 0.03$ & 2005 Feb 24 \\
\hline J04333678+2609492 & IS Tau $\mathrm{A}+\mathrm{B}$ & $7.68 \pm 0.02$ & $7.23 \pm 0.02$ & $6.83 \pm 0.03$ & $6.03 \pm 0.03$ & 2004 Mar 7 \\
\hline & & $7.85 \pm 0.02$ & $7.41 \pm 0.02$ & $6.95 \pm 0.03$ & $6.00 \pm 0.03$ & 2005 Feb 24 \\
\hline $\mathrm{J} 04333905+2227207$ & & $9.97 \pm 0.02$ & $9.72 \pm 0.02$ & $9.54 \pm 0.03$ & $9.17 \pm 0.03$ & 2007 Apr 3 \\
\hline J04333906+2520382 & DL Tau & $6.67 \pm 0.02$ & $6.10 \pm 0.02$ & $5.60 \pm 0.03$ & $4.83 \pm 0.03$ & 2004 Mar 7 \\
\hline & & $7.02 \pm 0.02$ & $6.30 \pm 0.02$ & $5.84 \pm 0.03$ & $5.07 \pm 0.03$ & 2005 Feb 24 \\
\hline J04333935+1751523 & HN Tau A+B & $6.92 \pm 0.02$ & $6.22 \pm 0.02$ & $5.62 \pm 0.03$ & $4.70 \pm 0.03$ & 2005 Feb 19 \\
\hline J04334171+1750402 & $\ldots$ & $9.97 \pm 0.02$ & $9.79 \pm 0.02$ & $9.56 \pm 0.03$ & $9.07 \pm 0.03$ & 2005 Feb 19 \\
\hline $\mathrm{J} 04334291+2526470$ & & out & $12.60 \pm 0.02$ & out & $12.56 \pm 0.05$ & 2004 Mar 7 \\
\hline & & $12.69 \pm 0.02$ & $12.56 \pm 0.02$ & $12.47 \pm 0.04$ & $12.45 \pm 0.05$ & 2005 Feb 24 \\
\hline J04334465+2615005 & $\cdots$ & $8.67 \pm 0.02$ & $8.22 \pm 0.02$ & $7.83 \pm 0.03$ & $7.20 \pm 0.03$ & 2004 Mar 7 \\
\hline & & $8.80 \pm 0.02$ & $8.25 \pm 0.02$ & $7.76 \pm 0.03$ & $7.14 \pm 0.03$ & 2007 Oct 16 \\
\hline J04334871+1810099 & DM Tau & $9.35 \pm 0.02$ & $9.30 \pm 0.02$ & $9.25 \pm 0.03$ & $8.70 \pm 0.03$ & 2005 Feb 19 \\
\hline $\mathrm{J} 04335200+2250301$ & CI Tau & $6.76 \pm 0.02$ & $6.27 \pm 0.02$ & $5.82 \pm 0.03$ & $4.92 \pm 0.03$ & 2005 Feb 20 \\
\hline & & $7.03 \pm 0.02$ & $6.38 \pm 0.02$ & $6.09 \pm 0.03$ & $5.37 \pm 0.03$ & 2007 Apr 3 \\
\hline J04335245+2612548 & $\cdots$ & $13.13 \pm 0.02$ & $12.67 \pm 0.02$ & $12.24 \pm 0.04$ & $11.49 \pm 0.05$ & 2004 Mar 7 \\
\hline & & $13.07 \pm 0.02$ & $12.64 \pm 0.02$ & $12.25 \pm 0.04$ & $11.42 \pm 0.04$ & 2005 Feb 22 \\
\hline & & $13.18 \pm 0.02$ & $12.70 \pm 0.02$ & $12.28 \pm 0.04$ & $11.52 \pm 0.04$ & 2007 Oct 16 \\
\hline J04335252+2256269 & XEST 17-059 & out & $8.65 \pm 0.02$ & out & $8.62 \pm 0.03$ & 2005 Feb 20 \\
\hline & & $8.77 \pm 0.02$ & out & $8.59 \pm 0.03$ & out & 2005 Feb 24 \\
\hline & & $8.80 \pm 0.02$ & $8.62 \pm 0.02$ & $8.61 \pm 0.03$ & $8.57 \pm 0.03$ & 2007 Apr 3 \\
\hline $\mathrm{J} 04335470+2613275$ & IT Tau B & $9.25 \pm 0.10$ & $8.68 \pm 0.10$ & $8.26 \pm 0.10$ & $7.46 \pm 0.10$ & 2004 Mar 7 \\
\hline
\end{tabular}


TABLE $4-$ Continued

\begin{tabular}{|c|c|c|c|c|c|c|}
\hline $2 \mathrm{MASS}^{\mathrm{a}}$ & Name & {$[3.6]$} & {$[4.5]$} & {$[5.8]$} & {$[8.0]$} & Date \\
\hline \multirow{5}{*}{ J04335470+2613275 } & \multirow{5}{*}{ IT Tau A } & $9.21 \pm 0.10$ & $8.63 \pm 0.10$ & $8.09 \pm 0.10$ & $7.41 \pm 0.10$ & 2005 Feb 23 \\
\hline & & $8.95 \pm 0.10$ & $8.61 \pm 0.10$ & $8.08 \pm 0.10$ & $7.46 \pm 0.10$ & 2007 Oct 16 \\
\hline & & $7.25 \pm 0.02$ & $6.89 \pm 0.02$ & $6.57 \pm 0.03$ & $5.96 \pm 0.03$ & 2004 Mar 7 \\
\hline & & $7.22 \pm 0.02$ & $6.84 \pm 0.02$ & $6.53 \pm 0.03$ & $5.96 \pm 0.03$ & 2005 Feb 22 \\
\hline & & $7.27 \pm 0.02$ & $6.88 \pm 0.02$ & $6.50 \pm 0.03$ & $5.91 \pm 0.03$ & 2007 Oct 16 \\
\hline J04335546+1838390 & J2-2041 & $9.39 \pm 0.02$ & $9.37 \pm 0.02$ & $9.32 \pm 0.03$ & $9.29 \pm 0.03$ & 2005 Feb 19 \\
\hline \multirow{3}{*}{ J04341099+2251445 } & JH 108 & $9.28 \pm 0.02$ & $9.22 \pm 0.02$ & $9.20 \pm 0.03$ & $9.14 \pm 0.03$ & 2005 Feb 20 \\
\hline & & $9.29 \pm 0.02$ & out & $9.10 \pm 0.03$ & out & 2005 Feb 24 \\
\hline & & $9.25 \pm 0.02$ & $9.25 \pm 0.02$ & $9.18 \pm 0.03$ & $9.17 \pm 0.03$ & 2007 Apr 3 \\
\hline \multirow[t]{2}{*}{ J04341527+2250309 } & CFHT 1 & $11.22 \pm 0.02$ & $11.07 \pm 0.02$ & $10.95 \pm 0.03$ & $10.98 \pm 0.03$ & 2005 Feb 20 \\
\hline & & $11.17 \pm 0.02$ & $11.04 \pm 0.02$ & $10.96 \pm 0.03$ & $10.99 \pm 0.03$ & 2007 Apr 3 \\
\hline J04341803+1830066 & HBC 407 & $9.82 \pm 0.02$ & $9.79 \pm 0.02$ & $9.81 \pm 0.03$ & $9.76 \pm 0.03$ & 2005 Feb 19 \\
\hline J04344544+2308027 & & $11.24 \pm 0.02$ & $11.19 \pm 0.02$ & $11.09 \pm 0.03$ & $11.08 \pm 0.03$ & 2005 Feb 24 \\
\hline \multirow[t]{2}{*}{ J04345542+2428531 } & AA Tau & $7.23 \pm 0.02$ & $6.76 \pm 0.02$ & $6.36 \pm 0.03$ & $5.61 \pm 0.03$ & 2005 Feb 20 \\
\hline & & $7.27 \pm 0.02$ & $6.78 \pm 0.02$ & $6.40 \pm 0.03$ & $5.58 \pm 0.03$ & 2005 Feb 21 \\
\hline \multirow[t]{2}{*}{ J04345693+2258358 } & XEST 08-003 & $9.06 \pm 0.02$ & out & $8.89 \pm 0.03$ & out & 2005 Feb 24 \\
\hline & & $9.05 \pm 0.02$ & $8.99 \pm 0.02$ & $8.94 \pm 0.03$ & $8.94 \pm 0.03$ & 2007 Apr 3 \\
\hline J04350850+2311398 & & $11.19 \pm 0.02$ & $11.06 \pm 0.02$ & $11.02 \pm 0.03$ & $10.98 \pm 0.03$ & 2005 Feb 24 \\
\hline \multirow{2}{*}{$\mathrm{J} 04352020+2232146$} & HO Tau & $8.97 \pm 0.02$ & $8.58 \pm 0.02$ & $8.46 \pm 0.03$ & $7.82 \pm 0.03$ & 2005 Feb 20 \\
\hline & & $8.88 \pm 0.02$ & $8.49 \pm 0.02$ & $8.36 \pm 0.03$ & $7.72 \pm 0.03$ & 2007 Apr 3 \\
\hline J04352089+2254242 & FF Tau A+B & $8.41 \pm 0.02$ & $8.37 \pm 0.02$ & $8.35 \pm 0.03$ & $8.31 \pm 0.03$ & 2005 Feb 20 \\
\hline & & $8.31 \pm 0.02$ & out & $8.21 \pm 0.03$ & out & 2005 Feb 24 \\
\hline & & $8.37 \pm 0.02$ & $8.37 \pm 0.02$ & $8.36 \pm 0$ & $8.35 \pm 0.03$ & 2007 Apr 3 \\
\hline J04352450+1751429 & HBC $412 \mathrm{~A}+\mathrm{B}$ & $8.91 \pm 0.02$ & $8.88 \pm 0.02$ & $8.83 \pm 0.03$ & $8.80 \pm 0.03$ & 2005 Feb 19 \\
\hline J04352737+2414589 & DN Tau & $7.47 \pm 0.02$ & $7.14 \pm 0.02$ & $6.74 \pm 0.03$ & $6.00 \pm 0.03$ & 2005 Feb 20 \\
\hline & & $7.43 \pm 0.02$ & $7.16 \pm 0.02$ & $6.71 \pm 0.03$ & $6.00 \pm 0.03$ & 2005 Feb 21 \\
\hline & & sat & sat & $6.54 \pm 0.03$ & sat & 2006 Mar 25 \\
\hline$\ldots$ & IRAS $04325+2402 \mathrm{C}$ & $13.50 \pm 0.30$ & $13.10 \pm 0.20$ & $12.60 \pm 0.20$ & $12.00 \pm 0.20$ & 2005 Feb 20 \\
\hline & & $13.50 \pm 0.30$ & $12.90 \pm 0.20$ & $12.60 \pm 0.20$ & $12.00 \pm 0.20$ & 2005 Feb 21 \\
\hline & & $13.40 \pm 0.30$ & $12.90 \pm 0.20$ & $12.40 \pm 0.20$ & $11.70 \pm 0.20$ & 2006 Mar 25 \\
\hline J04353539+2408194 & IRAS $04325+2402 \mathrm{~A}+\mathrm{B}$ & $9.89 \pm 0.02$ & $9.26 \pm 0.02$ & $8.99 \pm 0.03$ & $8.49 \pm 0.03$ & 2005 Feb 20 \\
\hline & & $9.85 \pm 0.02$ & $9.18 \pm 0.02$ & $8.96 \pm 0.03$ & $8.46 \pm 0.03$ & 2005 Feb 21 \\
\hline & & $9.90 \pm 0.02$ & $9.34 \pm 0.02$ & 9.12 & $8.52 \pm 0.03$ & 2006 Mar 25 \\
\hline J04354093+2411087 & CoKu Tau 3 A+B & $7.28 \pm 0.02$ & $6.83 \pm 0.02$ & $6.33 \pm 0.03$ & $5.59 \pm 0.03$ & 2005 Feb 20 \\
\hline & & $7.35 \pm 0.02$ & $6.87 \pm 0.02$ & $6.42 \pm 0.03$ & $5.57 \pm 0.03$ & 2005 Feb 21 \\
\hline J04354183+2234115 & KPNO 8 & $11.60 \pm 0.02$ & $11.46 \pm 0.02$ & $11.44 \pm 0.03$ & $11.39 \pm 0.03$ & 2005 Feb 20 \\
\hline & & $11.60 \pm 0.02$ & $11.47 \pm 0.02$ & $11.41 \pm 0.03$ & $11.45 \pm 0.04$ & 2007 Apr 3 \\
\hline J04354203+2252226 & XEST 08-033 & $9.67 \pm 0.02$ & out & $9.54 \pm 0.03$ & out & 2005 Feb 21 \\
\hline & & $9.63 \pm 0.02$ & $9.48 \pm 0.02$ & & $9.50 \pm 0.03$ & 2007 Apr 3 \\
\hline J04354526+2737130 & & $13.12 \pm 0.02$ & $13.04 \pm 0.02$ & $12.89 \pm 0.04$ & $13.04 \pm 0.07$ & 2005 Feb 22 \\
\hline J04354733+2250216 & HQ Tau & $6.73 \pm 0.02$ & $6.26 \pm 0.02$ & $5.74 \pm 0.03$ & $4.60 \pm 0.03$ & 2005 Feb 20 \\
\hline & & $6.81 \pm 0.02$ & out & & out & 2005 Feb 21 \\
\hline & & $6.42 \pm 0.02$ & $5.84 \pm 0.02$ & $5.36 \pm 0.03$ & $4.38 \pm 0.03$ & 2007 Apr 3 \\
\hline J04355109+2252401 & KPNO 15 & $9.73 \pm 0.02$ & $9.74 \pm 0.02$ & $9.60 \pm 0.03$ & $9.67 \pm 0.03$ & 2005 Feb 20 \\
\hline & & $9.74 \pm 0.02$ & out & $9.66 \pm 0.03$ & out & 2005 Feb 21 \\
\hline & & $9.76 \pm 0.02$ & $9.69 \pm 0.02$ & $9.64 \pm 0$ & $9.63 \pm 0.03$ & 2007 Apr 3 \\
\hline J04355143+2249119 & KPNO 9 & $13.61 \pm 0.02$ & $13.53 \pm 0.02$ & $13.44 \pm 0.05$ & $13.48 \pm 0.08$ & 2005 Feb 20 \\
\hline & & $13.63=$ & out & out & out & 2005 Feb 21 \\
\hline & & $13.60=$ & $13.47 \pm 0.03$ & $13.56 \pm 0.07$ & $13.35 \pm 0.10$ & 2007 Apr 3 \\
\hline J04355209+2255039 & XEST 08-047 & $9.54 \pm 0.02$ & $9.44 \pm 0.02$ & $9.38=$ & $9.37 \pm 0.03$ & 2005 Feb 20 \\
\hline & & $9.53 \pm 0.02$ & out & & out & 2005 Feb 21 \\
\hline & & $9.52 \pm 0.02$ & $9.43 \pm 0.02$ & $9.39 \pm 0.03$ & $9.36 \pm 0.03$ & 2007 Apr 3 \\
\hline J04355277+2254231 & HP Tau & $6.51 \pm 0.02$ & $6.03 \pm 0.02$ & $5.58 \pm 0.03$ & $4.59 \pm 0.03$ & 2005 Feb 20 \\
\hline & & & out & & out & 2005 Feb 21 \\
\hline & & $6.58 \pm 0.02$ & $6.13 \pm 0.02$ & $5.73 \pm 0.03$ & $4.80 \pm 0.03$ & 2007 Apr 3 \\
\hline J04355286+2250585 & XEST 08-049 & $9.43 \pm 0.02$ & $9.31 \pm 0.02$ & $9.33 \pm 0.03$ & $9.27 \pm 0.03$ & 2005 Feb 20 \\
\hline & & $9.41 \pm 0.02$ & out & $9.28 \pm 0.03$ & out & 2005 Feb 21 \\
\hline & & $9.42 \pm 0.02$ & $9.29 \pm 0.02$ & $9.24 \pm 0.03$ & $9.29 \pm 0.03$ & 2007 Apr 3 \\
\hline J04355349+2254089 & HP Tau/G3 & $8.58 \pm 0.02$ & $8.53 \pm 0.02$ & & $8.48 \pm 0.03$ & 2005 Feb 20 \\
\hline & & $8.56 \pm 0.02$ & out & $8.51 \pm 0.03$ & out & 2005 Feb 21 \\
\hline & & $8.61 \pm 0.02$ & $8.52 \pm 0.02$ & $8.47 \pm 0.03$ & $8.48 \pm 0.03$ & 2007 Apr 3 \\
\hline J04355415+2254134 & HP Tau/G2 & $7.14 \pm 0.02$ & $7.11 \pm 0.02$ & $7.04 \pm 0.03$ & $7.03 \pm 0.03$ & 2005 Feb 20 \\
\hline & & & out & & out & 2005 Feb 21 \\
\hline & & $7.16 \pm 0.02$ & $7.14 \pm 0.02$ & $7.05 \pm 0.03$ & $7.00 \pm 0.03$ & 2007 Apr 3 \\
\hline J04355684+2254360 & Haro 6-28 A+B & $8.70 \pm 0.02$ & $8.23 \pm 0.02$ & & $7.15 \pm 0.03$ & 2005 Feb 20 \\
\hline & & & out & & out & 2005 Feb 21 \\
\hline & & $8.63 \pm 0.02$ & $8.17 \pm 0.02$ & $7.79 \pm 0.03$ & $7.14 \pm 0.03$ & 2007 Apr 3 \\
\hline & XEST 09-042 & $8.11 \pm 0.02$ & $8.18 \pm 0.02$ & & $8.06 \pm 0.03$ & 2007 Apr 3 \\
\hline J04361030+2159364 & & $12.96 \pm 0.02$ & $12.66 \pm 0.02$ & $12.35 \pm 0.03$ & $11.72 \pm 0.03$ & 2007 Mar 28 \\
\hline & & $12.99 \pm 0.02$ & $12.70 \pm 0.02$ & $12.37 \pm 0.04$ & $11.79 \pm 0.04$ & 2007 Apr 3 \\
\hline J04361038+2259560 & CFHT 2 & $11.57 \pm 0.02$ & $11.38 \pm 0.02$ & $11.37 \pm 0.03$ & $11.36 \pm 0.03$ & 2005 Feb 20 \\
\hline & & $11.62 \pm 0.02$ & $11.39 \pm 0.02$ & $11.30 \pm 0.03$ & $11.32 \pm 0.03$ & 2005 Feb 21 \\
\hline & & & $11.37 \pm 0.02$ & $11.38 \pm 0.03$ & $11.31 \pm 0.04$ & 2007 Apr 3 \\
\hline J04361909+2542589 & $\mathrm{LkCa} 14$ & $8.53 \pm 0.02$ & $8.51 \pm 0.02$ & $8.47 \pm 0.03$ & $8.45 \pm 0.03$ & 2004 Mar 7 \\
\hline & & & $8.47 \pm 0.02$ & & $8.44 \pm 0.03$ & 2005 Feb 22 \\
\hline J04362151+2351165 & & $11.87 \pm 0.02$ & $11.47 \pm 0.02$ & $11.12 \pm 0.03$ & $10.55 \pm 0.03$ & 2005 Feb 21 \\
\hline J04363893+2258119 & CFHT 3 & $11.77 \pm 0.02$ & $11.62 \pm 0.02$ & $11.56 \pm 0.03$ & $11.54 \pm 0.03$ & 2005 Feb 20 \\
\hline
\end{tabular}


TABLE $4-$ Continued

\begin{tabular}{|c|c|c|c|c|c|c|}
\hline $2 \mathrm{MASS}^{\mathrm{a}}$ & Name & {$[3.6]$} & {$[4.5]$} & {$[5.8]$} & {$[8.0]$} & Date \\
\hline & & $11.77 \pm 0.02$ & $11.65 \pm 0.02$ & $11.68 \pm 0.03$ & $11.59 \pm 0.04$ & 2005 Feb 21 \\
\hline & & $11.77 \pm 0.02$ & $11.78 \pm 0.02$ & $11.53 \pm 0.03$ & $11.53 \pm 0.04$ & 2007 Apr 3 \\
\hline J04373705+2331080 & & $14.16 \pm 0.02$ & $13.88 \pm 0.03$ & $13.59 \pm 0.05$ & $13.30 \pm 0.08$ & 2005 Feb 21 \\
\hline J04375670+2546229 & ITG 1 & $11.95 \pm 0.02$ & $11.47 \pm 0.02$ & $10.71 \pm 0.03$ & $9.89 \pm 0.03$ & 2005 Feb 22 \\
\hline J04380083+2558572 & ITG 2 & $9.57 \pm 0.02$ & $9.42 \pm 0.02$ & $9.33 \pm 0.03$ & $9.34 \pm 0.03$ & 2005 Feb 22 \\
\hline J04381486+2611399 & $\ldots$ & $10.79 \pm 0.02$ & $10.14 \pm 0.02$ & $9.61 \pm 0.03$ & $8.91 \pm 0.03$ & 2005 Feb 22 \\
\hline $\mathrm{J} 04381630+2326402$ & $\ldots$ & out & out & out & out & ... \\
\hline \multirow[t]{2}{*}{ J04382134+2609137 } & GM Tau & $9.44 \pm 0.02$ & $8.95 \pm 0.02$ & $8.64 \pm 0.03$ & $7.97 \pm 0.03$ & 2004 Mar 7 \\
\hline & & $9.23 \pm 0.02$ & $8.73 \pm 0.02$ & $8.40 \pm 0.03$ & $7.83 \pm 0.03$ & 2005 Feb 22 \\
\hline \multirow[t]{2}{*}{ J04382858+2610494 } & DO Tau & $6.11 \pm 0.02$ & $5.50 \pm 0.02$ & $5.00 \pm 0.03$ & $4.23 \pm 0.03$ & 2004 Mar 7 \\
\hline & & $6.19 \pm 0.02$ & $5.60 \pm 0.02$ & $5.14 \pm 0.03$ & $4.39 \pm 0.03$ & 2005 Feb 22 \\
\hline \multirow[t]{2}{*}{ J04383528+2610386 } & HV Tau A+B & $7.65 \pm 0.02$ & $7.54 \pm 0.02$ & $7.46 \pm 0.03$ & $7.43 \pm 0.03$ & 2004 Mar 7 \\
\hline & & $7.71 \pm 0.02$ & $7.55 \pm 0.02$ & $7.44 \pm 0.03$ & $7.43 \pm 0.03$ & 2005 Feb 22 \\
\hline \multirow[t]{2}{*}{$\cdots$} & HV Tau C & $11.37 \pm 0.05$ & $10.74 \pm 0.05$ & $10.20 \pm 0.05$ & $9.27 \pm 0.05$ & 2004 Mar 7 \\
\hline & & $11.29 \pm 0.05$ & $10.73 \pm 0.05$ & $10.21 \pm 0.05$ & $9.35 \pm 0.05$ & 2005 Feb 23 \\
\hline J04385859+2336351 & $\ldots$ & $10.49 \pm 0.02$ & out & $9.84 \pm 0.03$ & out & 2005 Feb 21 \\
\hline J04385871+2323595 & $\ldots$ & out & out & out & out & $\ldots$ \\
\hline J04390163+2336029 & $\ldots$ & $9.72 \pm 0.02$ & out & $9.17 \pm 0.03$ & out & 2005 Feb 21 \\
\hline J04390396+2544264 & $\ldots$ & $10.78 \pm 0.02$ & $10.37 \pm 0.02$ & $10.03 \pm 0.03$ & $9.16 \pm 0.03$ & 2005 Feb 22 \\
\hline J04390525+2337450 & $\ldots$ & $10.54 \pm 0.02$ & $10.14 \pm 0.02$ & $9.73 \pm 0.03$ & $9.02 \pm 0.03$ & 2005 Feb 21 \\
\hline $\mathrm{J} 04390637+2334179$ & $\ldots$ & $10.79 \pm 0.02$ & out & $10.69 \pm 0.03$ & out & 2005 Feb 21 \\
\hline \multirow[t]{2}{*}{ J04391389+2553208 } & IRAS $04361+2547$ & $8.54 \pm 0.02$ & $7.62 \pm 0.02$ & $7.14 \pm 0.03$ & $5.88 \pm 0.03$ & 2004 Mar 7 \\
\hline & & $7.95 \pm 0.02$ & $7.01 \pm 0.02$ & $6.42 \pm 0.03$ & $4.77 \pm 0.03$ & 2005 Feb 22 \\
\hline $\mathrm{J} 04391741+2247533$ & VY Tau A+B & $8.61 \pm 0.02$ & $8.42 \pm 0.02$ & $8.10 \pm 0.03$ & $7.42 \pm 0.03$ & 2005 Feb 20 \\
\hline \multirow{2}{*}{ J04391779+2221034 } & $\mathrm{LkCa} 15$ & $7.57 \pm 0.02$ & $7.34 \pm 0.02$ & $7.18 \pm 0.03$ & $6.50 \pm 0.03$ & 2005 Feb 20 \\
\hline & & $7.70 \pm 0.02$ & $7.35 \pm 0.02$ & $7.28 \pm 0.03$ & $6.59 \pm 0.03$ & 2007 Apr 3 \\
\hline \multirow[t]{2}{*}{ J04392090+2545021 } & GN Tau A+B & $7.09 \pm 0.02$ & $6.61 \pm 0.02$ & $6.30 \pm 0.03$ & $5.59 \pm 0.03$ & 2004 Mar 7 \\
\hline & & $7.00 \pm 0.02$ & $6.50 \pm 0.02$ & $6.20 \pm 0.03$ & $5.38 \pm 0.03$ & 2005 Feb 22 \\
\hline J04393364+2359212 & & $9.61 \pm 0.02$ & $9.16 \pm 0.02$ & $8.82 \pm 0.03$ & $7.91 \pm 0.03$ & 2005 Feb 21 \\
\hline J04393519+2541447 & IRAS $04365+2535$ & $7.54 \pm 0.02$ & $6.07 \pm 0.02$ & $5.09 \pm 0.03$ & $4.37 \pm 0.03$ & 2004 Mar 7 \\
\hline & & $7.16 \pm 0.02$ & $5.73 \pm 0.02$ & $4.81 \pm 0.03$ & $4.08 \pm 0.03$ & 2005 Feb 22 \\
\hline J04394488+2601527 & ITG 15 & $8.41 \pm 0.02$ & $8.14 \pm 0.02$ & $7.72 \pm 0.03$ & $7.08 \pm 0.03$ & 2004 Mar 7 \\
\hline & & $8.45 \pm 0.02$ & $8.12 \pm 0.02$ & $7.69 \pm 0.03$ & $7.11 \pm 0.03$ & 2005 Feb 22 \\
\hline J04394748+2601407 & CFHT 4 & $9.51 \pm 0.02$ & $9.08 \pm 0.02$ & $8.62 \pm 0.03$ & $7.85 \pm 0.03$ & 2004 Mar 7 \\
\hline & & $9.47 \pm 0.02$ & $9.04 \pm 0.02$ & $8.59 \pm 0.03$ & $7.80 \pm 0.03$ & 2005 Feb 22 \\
\hline$\ldots$ & IRAS $04368+2557$ & $13.23 \pm 0.09$ & $10.87 \pm 0.04$ & $9.67 \pm 0.04$ & $9.34 \pm 0.04$ & 2004 Mar 7 \\
\hline & & $13.51 \pm 0.10$ & $11.15 \pm 0.05$ & $9.95 \pm 0.04$ & $9.56 \pm 0.04$ & 2005 Feb 22 \\
\hline J04395574+2545020 & IC2087IR & sat & sat & $3.33 \pm 0.03$ & $2.76 \pm 0.03$ & 2004 Mar 7 \\
\hline & & sat & sat & $3.43 \pm 0.03$ & $2.75 \pm 0.03$ & 2005 Feb 22 \\
\hline J04400067+2358211 & $\ldots$ & $10.90 \pm 0.02$ & $10.61 \pm 0.02$ & $10.35 \pm 0.03$ & $9.69 \pm 0.03$ & 2005 Feb 21 \\
\hline J04400174+2556292 & $\cdots$ & $10.07 \pm 0.02$ & out & $9.81 \pm 0.03$ & out & 2004 Mar 7 \\
\hline & & $10.13 \pm 0.02$ & $9.91 \pm 0.02$ & $9.84 \pm 0.03$ & $9.81 \pm 0.03$ & 2005 Feb 22 \\
\hline J04400800+2605253 & IRAS $04370+2559$ & $7.87 \pm 0.02$ & $7.28 \pm 0.02$ & $6.84 \pm 0.03$ & $5.84 \pm 0.03$ & 2004 Mar 7 \\
\hline & & $7.92 \pm 0.02$ & $7.30 \pm 0.02$ & $6.92 \pm 0.03$ & $5.91 \pm 0.03$ & 2005 Feb 22 \\
\hline J04403979+2519061 & $\ldots$ & $9.79 \pm 0.02$ & $9.61 \pm 0.02$ & $9.62 \pm 0.03$ & $9.57 \pm 0.03$ & 2005 Feb 21 \\
\hline J04404950+2551191 & JH 223 & $8.87 \pm 0.02$ & $8.55 \pm 0.02$ & $8.23 \pm 0.03$ & $7.72 \pm 0.03$ & 2004 Feb 9 \\
\hline & & sat & $8.58 \pm 0.02$ & $8.35 \pm 0.03$ & $7.72 \pm 0.03$ & 2004 Oct 8 \\
\hline & & $8.86 \pm 0.02$ & $8.47 \pm 0.02$ & $8.24 \pm 0.03$ & $7.62 \pm 0.03$ & 2005 Feb 23 \\
\hline & & $8.85 \pm 0.02$ & $8.50 \pm 0.02$ & $8.28 \pm 0.03$ & $7.75 \pm 0.03$ & 2007 Oct 16 \\
\hline J04410424+2557561 & Haro 6-32 & $9.57 \pm 0.02$ & $9.49 \pm 0.02$ & $9.42 \pm 0.03$ & $9.52 \pm 0.03$ & 2004 Sep 8 \\
\hline & & $9.60 \pm 0.02$ & $9.49 \pm 0.02$ & $9.47 \pm 0.03$ & $9.47 \pm 0.03$ & 2004 Oct 8 \\
\hline & & $9.58 \pm 0.02$ & $9.47 \pm 0.02$ & $9.48 \pm 0.03$ & $9.44 \pm 0.03$ & 2005 Feb 23 \\
\hline J04410470+2451062 & IW Tau A+B & $8.11 \pm 0.02$ & $8.09 \pm 0.02$ & $8.02 \pm 0.03$ & $7.99 \pm 0.03$ & 2004 Feb 9 \\
\hline & & $8.10 \pm 0.02$ & $8.12 \pm 0.02$ & $8.06 \pm 0.03$ & $8.03 \pm 0.03$ & 2007 Oct 16 \\
\hline J04410826+2556074 & ITG 33A & $9.79 \pm 0.02$ & $9.19 \pm 0.02$ & $8.62 \pm 0.03$ & $7.82 \pm 0.03$ & 2004 Sep 8 \\
\hline & & $9.67 \pm 0.02$ & $9.07 \pm 0.02$ & $8.54 \pm 0.03$ & $7.77 \pm 0.03$ & 2004 Oct 8 \\
\hline & & $9.61 \pm 0.02$ & $9.04 \pm 0.02$ & $8.48 \pm 0.03$ & $7.65 \pm 0.03$ & 2005 Feb 23 \\
\hline J04411078+2555116 & ITG 34 & out & $10.23 \pm 0.02$ & out & $9.14 \pm 0.03$ & 2004 Feb 9 \\
\hline & & $10.70 \pm 0.02$ & $10.21 \pm 0.02$ & $9.90 \pm 0.03$ & $9.20 \pm 0.03$ & 2004 Sep 8 \\
\hline & & $10.82 \pm 0.02$ & $10.29 \pm 0.02$ & $9.91 \pm 0.03$ & $9.15 \pm 0.03$ & 2004 Oct 8 \\
\hline & & $10.75 \pm 0.02$ & $10.28 \pm 0.02$ & $9.89 \pm 0.03$ & $9.25 \pm 0.03$ & 2005 Feb 23 \\
\hline J04411267+2546354 & IRAS $04381+2540$ & $9.18 \pm 0.02$ & $7.85 \pm 0.02$ & $6.84 \pm 0.03$ & $5.83 \pm 0.03$ & 2004 Feb 9 \\
\hline & & $9.11 \pm 0.02$ & $7.77 \pm 0.02$ & $6.74 \pm 0.03$ & $5.67 \pm 0.03$ & 2005 Feb 23 \\
\hline $\mathrm{J} 04411681+2840000$ & $\mathrm{CoKu} \mathrm{Tau} / 4$ & $8.47 \pm 0.02$ & $8.39 \pm 0.02$ & $8.34 \pm 0.03$ & $8.13 \pm 0.03$ & 2004 Feb 9 \\
\hline J04412464+2543530 & ITG 40 & $10.37 \pm 0.02$ & $9.83 \pm 0.02$ & $9.33 \pm 0.03$ & $8.88 \pm 0.03$ & 2004 Oct 8 \\
\hline & & $10.48 \pm 0.02$ & $9.78 \pm 0.02$ & $9.40 \pm 0.03$ & $8.87 \pm 0.03$ & 2005 Feb 23 \\
\hline J04413882+2556267 & IRAS $04385+2550$ & $8.12 \pm 0.02$ & $7.54 \pm 0.02$ & $7.04 \pm 0.03$ & $6.03 \pm 0.03$ & 2004 Feb 9 \\
\hline & & & & $6.69 \pm 0.03$ & $5.58 \pm 0.03$ & 2004 Oct 8 \\
\hline & & $8.21 \pm 0.02$ & $7.74 \pm 0.02$ & $7.09 \pm 0.03$ & $6.09 \pm 0.03$ & 2005 Feb 23 \\
\hline J04414489+2301513 & $\ldots$ & $12.26 \pm 0.02$ & $11.88 \pm 0.02$ & $11.53 \pm 0.03$ & $11.00 \pm 0.03$ & 2007 Mar 28 \\
\hline J04414565+2301580 & $\ldots$ & sat & $9.48 \pm 0.02$ & $9.37 \pm 0.03$ & $9.22 \pm 0.03$ & 2007 Mar 28 \\
\hline J04414825+2534304 & & out & $10.87 \pm 0.02$ & out & $9.59 \pm 0.03$ & 2004 Oct 8 \\
\hline & & $11.38 \pm 0.02$ & $10.87 \pm 0.02$ & $10.47 \pm 0.03$ & $9.54 \pm 0.03$ & 2005 Feb 23 \\
\hline J04420548+2522562 & LkHa $332 / G 2 A+B$ & $7.94 \pm 0.02$ & $7.87 \pm 0.02$ & $7.75 \pm 0.03$ & $7.69 \pm 0.03$ & 2004 Feb 9 \\
\hline & & $7.91 \pm 0.02$ & $7.83 \pm 0.02$ & $7.73 \pm 0.03$ & $7.69 \pm 0.03$ & 2005 Feb 23 \\
\hline J04420732+2523032 & LkHa $332 / \mathrm{G} 1 \mathrm{~A}+\mathrm{B}$ & $7.66 \pm 0.02$ & $7.58 \pm 0.02$ & $7.50 \pm 0.03$ & $7.44 \pm 0.03$ & 2004 Feb 9 \\
\hline & & $7.59 \pm 0.02$ & $7.56 \pm 0.02$ & $7.48 \pm 0.03$ & $7.50 \pm 0.03$ & 2005 Feb 23 \\
\hline
\end{tabular}


Disk Population of Taurus

TABLE 4 - Continued

\begin{tabular}{|c|c|c|c|c|c|c|}
\hline $2 \mathrm{MASS}^{\mathrm{a}}$ & Name & {$[3.6]$} & {$[4.5]$} & {$[5.8]$} & {$[8.0]$} & Date \\
\hline \multirow[t]{2}{*}{ J04420777+2523118 } & V955 Tau A+B & $6.90 \pm 0.02$ & $6.46 \pm 0.02$ & $6.05 \pm 0.03$ & $5.34 \pm 0.03$ & 2004 Feb 9 \\
\hline & & $6.93 \pm 0.02$ & $6.53 \pm 0.02$ & $6.06 \pm 0.03$ & $5.33 \pm 0.03$ & 2005 Feb 23 \\
\hline \multirow[t]{2}{*}{ J04422101+2520343 } & CIDA 7 & $9.51 \pm 0.02$ & $9.11 \pm 0.02$ & $8.66 \pm 0.03$ & $7.81 \pm 0.03$ & 2004 Feb 9 \\
\hline & & $9.52 \pm 0.02$ & $9.18 \pm 0.02$ & $8.63 \pm 0.03$ & $7.79 \pm 0.03$ & 2005 Feb 23 \\
\hline \multirow[t]{2}{*}{ J04423769+2515374 } & DP Tau & $7.58 \pm 0.02$ & $6.89 \pm 0.02$ & $6.34 \pm 0.03$ & $5.34 \pm 0.03$ & 2004 Feb 9 \\
\hline & & $7.50 \pm 0.02$ & $6.85 \pm 0.02$ & $6.33 \pm 0.03$ & $5.36 \pm 0.03$ & 2005 Feb 23 \\
\hline \multirow[t]{2}{*}{ J04430309+2520187 } & GO Tau & $8.98 \pm 0.02$ & $8.62 \pm 0.02$ & $8.20 \pm 0.03$ & $7.40 \pm 0.03$ & 2004 Feb 9 \\
\hline & & $9.02 \pm 0.02$ & $8.61 \pm 0.02$ & $8.31 \pm 0.03$ & $7.42 \pm 0.03$ & 2005 Feb 23 \\
\hline \multirow[t]{2}{*}{ J04432023+2940060 } & CIDA 14 & $8.94 \pm 0.02$ & $8.64 \pm 0.02$ & $8.32 \pm 0.03$ & $7.65 \pm 0.03$ & 2004 Feb 9 \\
\hline & & $8.90 \pm 0.02$ & $8.60 \pm 0.02$ & $8.25 \pm 0.03$ & $7.53 \pm 0.03$ & 2004 Mar 11 \\
\hline \multirow[t]{2}{*}{ J04442713+2512164 } & IRAS $04414+2506$ & $9.75 \pm 0.02$ & $9.34 \pm 0.02$ & $8.87 \pm 0.03$ & $7.89 \pm 0.03$ & 2004 Feb 9 \\
\hline & & $9.52 \pm 0.02$ & $8.96 \pm 0.02$ & $8.33 \pm 0.03$ & $7.43 \pm 0.03$ & 2005 Feb 23 \\
\hline J04455129+1555496 & HD 30171 & $7.28 \pm 0.02$ & $7.24 \pm 0.02$ & $7.29 \pm 0.03$ & $7.24 \pm 0.03$ & 2004 Sep 7 \\
\hline J04455134+1555367 & IRAS $04429+1550$ & $8.55 \pm 0.02$ & $8.20 \pm 0.02$ & $7.80 \pm 0.03$ & $6.73 \pm 0.03$ & 2004 Sep 7 \\
\hline \multirow[t]{2}{*}{$\mathrm{J} 04464260+2459034$} & RXJ $04467+2459$ & $10.02 \pm 0.02$ & $9.92 \pm 0.02$ & $9.87 \pm 0.03$ & $9.89 \pm 0.03$ & 2004 Feb 9 \\
\hline & & $10.03 \pm 0.02$ & $9.92 \pm 0.02$ & $9.97 \pm 0.03$ & $9.91 \pm 0.03$ & 2005 Feb 24 \\
\hline J04465305+1700001 & DQ Tau & $7.05 \pm 0.02$ & $6.58 \pm 0.02$ & $6.07 \pm 0.03$ & $5.20 \pm 0.03$ & 2004 Mar 6 \\
\hline J04465897+1702381 & Haro 6-37 A & $6.64 \pm 0.04$ & $6.14 \pm 0.04$ & $5.80 \pm 0.05$ & $5.24 \pm 0.05$ & $2004 \operatorname{Mar} 6$ \\
\hline J04465897+1702381 & Haro 6-37 B & $7.85 \pm 0.07$ & $7.28 \pm 0.07$ & $7.12 \pm 0.07$ & $5.99 \pm 0.07$ & 2004 Mar 6 \\
\hline J04470620+1658428 & DR Tau & sat & $5.05 \pm 0.02$ & $4.47 \pm 0.03$ & $3.80 \pm 0.03$ & 2004 Mar 6 \\
\hline J04474859+2925112 & DS Tau & $7.36 \pm 0.02$ & $6.91 \pm 0.02$ & $6.65 \pm 0.03$ & $5.91 \pm 0.03$ & 2004 Feb 9 \\
\hline J04484189+1703374 & & $12.06 \pm 0.02$ & $11.94 \pm 0.02$ & $11.89 \pm 0.03$ & $11.88 \pm 0.03$ & 2006 Oct 26 \\
\hline J04514737+3047134 & UY Aur A+B & $6.08 \pm 0.02$ & $5.53 \pm 0.02$ & $4.98 \pm 0.03$ & $3.92 \pm 0.03$ & 2004 Feb 14 \\
\hline J04520668+3047175 & IRAS $04489+3042$ & $8.42 \pm 0.02$ & $7.68 \pm 0.02$ & $7.06 \pm 0.03$ & $6.17 \pm 0.03$ & 2004 Feb 14 \\
\hline J04551098+3021595 & GM Aur & $8.04 \pm 0.02$ & $7.88 \pm 0.02$ & $7.68 \pm 0.03$ & $7.07 \pm 0.03$ & 2004 Feb 14 \\
\hline \multirow[t]{3}{*}{ J04552333+3027366 } & & $11.49 \pm 0.02$ & out & $11.30 \pm 0.03$ & out & 2004 Feb 15 \\
\hline & & $11.51 \pm 0.02$ & $11.39 \pm 0.02$ & $11.37 \pm 0.03$ & $11.33 \pm 0.03$ & 2005 Feb 20 \\
\hline & & $11.51 \pm 0.02$ & $11.39 \pm 0.02$ & $11.34 \pm 0.03$ & bad & 2007 Mar 29 \\
\hline \multirow[t]{2}{*}{ J04553695+3017553 } & LkCa 19 & $8.12 \pm 0.02$ & $8.11 \pm 0.02$ & $8.06 \pm 0.03$ & $8.04 \pm 0.03$ & 2004 Feb 14 \\
\hline & & sat & sat & $8.03 \pm 0.03$ & $8.05 \pm 0.03$ & 2005 Feb 20 \\
\hline J04554046+3039057 & $\ldots$ & out & $11.31 \pm 0.02$ & out & $11.30 \pm 0.03$ & 2004 Feb 14 \\
\hline & & $11.45 \pm 0.02$ & $11.31 \pm 0.02$ & $11.27 \pm 0.03$ & $11.16 \pm 0.03$ & 2005 Feb 20 \\
\hline & & $11.44 \pm 0.02$ & out & $11.27 \pm 0.03$ & out & 2008 Nov 1 \\
\hline J04554535+3019389 & .. & $9.99 \pm 0.02$ & $9.63 \pm 0.02$ & $9.37 \pm 0.03$ & $8.77 \pm 0.03$ & 2004 Feb 14 \\
\hline & & $9.92 \pm 0.02$ & $9.62 \pm 0.02$ & $9.29 \pm 0.03$ & $8.74 \pm 0.03$ & 2005 Feb 20 \\
\hline & & out & $9.43 \pm 0.02$ & out & $8.65 \pm 0.03$ & 2008 Nov 1 \\
\hline J04554582+3033043 & AB Aur & sat & sat & $2.64 \pm 0.03$ & sat & $2004 \mathrm{Feb} 14$ \\
\hline J04554757+3028077 & & $9.63 \pm 0.02$ & out & $9.49 \pm 0.03$ & out & 2004 Feb 14 \\
\hline & & $9.60 \pm 0.02$ & $9.51 \pm 0.02$ & $9.48 \pm 0.03$ & $9.43 \pm 0.03$ & 2005 Feb 20 \\
\hline & & $9.65 \pm 0.02$ & $9.52 \pm 0.02$ & $9.50 \pm 0.03$ & $9.46 \pm 0.03$ & 2008 Nov 1 \\
\hline J04554801+3028050 & .. & $11.43 \pm 0.02$ & out & $10.78 \pm 0.03$ & out & 2004 Feb 14 \\
\hline & & $11.38 \pm 0.02$ & $11.00 \pm 0.02$ & $10.68 \pm 0.03$ & $10.02 \pm 0.03$ & 2005 Feb 20 \\
\hline & & $11.42 \pm 0.02$ & $11.09 \pm 0.02$ & $10.75 \pm 0.03$ & $10.09 \pm 0.03$ & 2008 Nov 1 \\
\hline J04554820+3030160 & XEST 26-052 & $10.64 \pm 0.02$ & $10.53 \pm 0.02$ & $10.52 \pm 0.03$ & $10.52 \pm 0.03$ & 2005 Feb 20 \\
\hline & & $10.67 \pm 0.02$ & $10.55 \pm 0.02$ & $10.56 \pm 0.03$ & $10.38 \pm 0.03$ & 2008 Nov 1 \\
\hline J04554969+3019400 & $\ldots$ & out & $11.21 \pm 0.02$ & out & out & 2004 Feb 14 \\
\hline & & $11.36 \pm 0.02$ & $11.15 \pm 0.02$ & $10.95 \pm 0.03$ & $10.62 \pm 0.03$ & 2005 Feb 20 \\
\hline & & out & $11.11 \pm 0.02$ & out & $10.66 \pm 0.03$ & 2008 Nov 1 \\
\hline J04555288+3006523 & & $10.39 \pm 0.02$ & $10.31 \pm 0.02$ & $10.26 \pm 0.03$ & $10.24 \pm 0.03$ & 2005 Feb 20 \\
\hline J04555605+3036209 & XEST 26-062 & $8.68 \pm 0.02$ & $8.36 \pm 0.02$ & $8.01 \pm 0.03$ & $7.16 \pm 0.03$ & $2004 \mathrm{Feb} 14$ \\
\hline & & out & $8.41 \pm 0.02$ & out & $7.18 \pm 0.03$ & 2005 Feb 20 \\
\hline & & $8.69 \pm 0.02$ & $8.37 \pm 0.02$ & $7.99 \pm 0.03$ & $7.20 \pm 0.03$ & 2008 Nov 1 \\
\hline J04555636+3049374 & & $10.75 \pm 0.02$ & $10.65 \pm 0.02$ & $10.58 \pm 0.03$ & $10.58 \pm 0.03$ & 2005 Feb 20 \\
\hline J04555938+3034015 & SU Aur & sat & $5.03 \pm 0.02$ & $4.58 \pm 0.03$ & $3.75 \pm 0.03$ & 2004 Feb 14 \\
\hline & & sat & sat & $4.52 \pm 0.03$ & $3.79 \pm 0.03$ & 2008 Nov 1 \\
\hline J04560118+3026348 & XEST26-071 & $9.42 \pm 0.02$ & $9.08 \pm 0.02$ & $8.68 \pm 0.03$ & $7.99 \pm 0.03$ & 2004 Feb 14 \\
\hline & & out & $9.02 \pm 0.02$ & out & $8.07 \pm 0.03$ & 2008 Nov 1 \\
\hline J04560201+3021037 & HBC 427 & $8.09 \pm 0.02$ & $8.10 \pm 0.02$ & $8.06 \pm 0.03$ & $8.02 \pm 0.03$ & 2004 Feb 14 \\
\hline & & sat & out & $7.98 \pm 0.03$ & out & 2005 Feb 20 \\
\hline & & out & $8.05 \pm 0.02$ & out & $8.02 \pm 0.03$ & 2008 Nov 1 \\
\hline J04574903+3015195 & $\cdots$ & $13.87 \pm 0.02$ & $13.76 \pm 0.02$ & $13.67 \pm 0.05$ & $13.69 \pm 0.10$ & 2005 Feb 20 \\
\hline & & $13.88 \pm 0.02$ & $13.76 \pm 0.02$ & $13.63 \pm 0.04$ & $13.82 \pm 0.06$ & 2006 Oct 26 \\
\hline J04584626+2950370 & MWC 480 & out & out & out & out & \\
\hline J05030659+2523197 & V836 Tau & $8.30 \pm 0.02$ & $7.93 \pm 0.02$ & $7.53 \pm 0.03$ & $6.82 \pm 0.03$ & 2004 Sep 7 \\
\hline J05044139+2509544 & CIDA 8 & $9.21 \pm 0.02$ & $8.88 \pm 0.02$ & $8.65 \pm 0.03$ & $8.00 \pm 0.03$ & $2004 \mathrm{Feb} 14$ \\
\hline & & $9.15 \pm 0.02$ & $8.86 \pm 0.02$ & $8.61 \pm 0.03$ & $7.96 \pm 0.03$ & 2004 Feb 16 \\
\hline J05052286+2531312 & CIDA 9 & $9.17 \pm 0.02$ & $8.56 \pm 0.02$ & $7.95 \pm 0.03$ & $7.02 \pm 0.03$ & 2004 Feb 14 \\
\hline & CIDA 10 & $9.59 \pm 0.02$ & $9.45 \pm 0.02$ & $9.44 \pm 0.03$ & $9.41 \pm 0.03$ & 2004 Feb 14 \\
\hline J05062332+2432199 & CIDA 11 & $9.10 \pm 0.02$ & $8.79 \pm 0.02$ & $8.47 \pm 0.03$ & $7.83 \pm 0.03$ & 2004 Feb 14 \\
\hline J05064662+2104296 & & $10.73 \pm 0.02$ & $10.64 \pm 0.02$ & $10.59 \pm 0.03$ & $10.57 \pm 0.03$ & 2008 Oct 31 \\
\hline J05071206+2437163 & RXJ $05072+243$ & $9.23 \pm 0.02$ & $9.24 \pm 0.02$ & $9.18 \pm 0.03$ & $9.14 \pm 0.03$ & 2004 Feb 14 \\
\hline J05074953+3024050 & RW Aur $A+B$ & $6.30 \pm 0.02$ & $5.70 \pm 0.02$ & $5.23 \pm 0.03$ & $4.32 \pm 0.03$ & 2004 Feb 12 \\
\hline J05075496+2500156 & CIDA 12 & $10.06 \pm 0.02$ & $9.84 \pm 0.02$ & $9.51 \pm 0.03$ & $8.72 \pm 0.03$ & 2004 Feb 14 \\
\hline
\end{tabular}


TABLE 4 - Continued

\begin{tabular}{lllllll}
\hline \hline $2 \mathrm{MASS}^{\mathrm{a}}$ & Name & {$[3.6]$} & {$[4.5]$} & {$[5.8]$} & {$[8.0]$} & Date \\
\hline
\end{tabular}

Note. - Entries of "...", "sat", "out", and "bad" indicate measurements that are absent because of non-detection, saturation, a position outside the field of view of the camera, and contamination by a cosmic ray, respectively.

a MASS Point Source Catalog.

TABLE 5

IRAC Photometry for Members of Taurus with Extended Emission

\begin{tabular}{|c|c|c|c|c|c|c|c|}
\hline $2 \mathrm{MASS}^{\mathrm{a}}$ & Name & Aperture $^{\mathrm{b}}$ & {$[3.6]$} & {$[4.5]$} & {$[5.8]$} & {$[8.0]$} & Date \\
\hline \multirow[t]{2}{*}{$\cdots$} & IRAS $04111+2800 \mathrm{G}$ & 15 & $12.60 \pm 0.05$ & $11.39 \pm 0.05$ & $10.79 \pm 0.05$ & $10.18 \pm 0.05$ & 2005 Feb 19 \\
\hline & & 15 & $12.74 \pm 0.05$ & $11.47 \pm 0.05$ & $10.70 \pm 0.05$ & $10.12 \pm 0.05$ & 2007 Mar 29 \\
\hline \multirow[t]{3}{*}{$\cdots$} & IRAS $04166+2706$ & 30 & $11.15 \pm 0.1$ & $10.07 \pm 0.1$ & $9.58 \pm 0.1$ & $9.17 \pm 0.1$ & 2005 Feb 20 \\
\hline & & 30 & $11.22 \pm 0.1$ & $10.07 \pm 0.1$ & $9.76 \pm 0.1$ & $9.13 \pm 0.1$ & 2005 Feb 21 \\
\hline & & 30 & $11.25 \pm 0.1$ & $10.13 \pm 0.1$ & $9.73 \pm 0.1$ & $9.37 \pm 0.1$ & 2007 Oct 17 \\
\hline \multirow[t]{3}{*}{ J04195844+2709570 } & IRAS $04169+2702$ & 30 & $8.32 \pm 0.05$ & $7.13 \pm 0.05$ & $\ldots$ & $\ldots$ & 2005 Feb 20 \\
\hline & & 30 & $8.29 \pm 0.05$ & out & $\ldots$ & $\ldots$ & 2005 Feb 21 \\
\hline & & 30 & $8.19 \pm 0.05$ & $7.03 \pm 0.05$ & $\ldots$ & $\ldots$ & 2007 Oct 17 \\
\hline \multirow[t]{2}{*}{$\ldots$} & IRAM $04191+1522$ & 15 & $13.79 \pm 0.05$ & $12.30 \pm 0.05$ & $11.74 \pm 0.1$ & $12.17 \pm 0.15$ & 2004 Sep 10 \\
\hline & & 15 & $14.06 \pm 0.05$ & $12.62 \pm 0.05$ & $12.14 \pm 0.05$ & $12.39 \pm 0.1$ & 2005 Sep 17 \\
\hline \multirow[t]{2}{*}{ J04275730+2619183 } & IRAS $04248+2612$ & 30 & $9.02 \pm 0.05$ & $8.38 \pm 0.05$ & $\ldots$ & $\ldots$ & 2004 Mar 7 \\
\hline & & 30 & $9.19 \pm 0.05$ & $8.65 \pm 0.05$ & $\ldots$ & $\ldots$ & 2005 Feb 22 \\
\hline \multirow[t]{3}{*}{$\cdots$} & L1521F-IRS & 15 & $13.95 \pm 0.1$ & $12.91 \pm 0.1$ & $12.68 \pm 0.2$ & $12.37 \pm 0.2$ & 2004 Sep 9 \\
\hline & & 15 & $14.25 \pm 0.1$ & $13.20 \pm 0.1$ & $13.13 \pm 0.3$ & $12.13 \pm 0.2$ & 2005 Feb 24 \\
\hline & & 15 & $14.08 \pm 0.1$ & $13.16 \pm 0.1$ & $12.62 \pm 0.1$ & $12.03 \pm 0.1$ & 2006 Mar 25 \\
\hline J04313407+1808049 & L1551/IRS5 & 30 & $6.74 \pm 0.05$ & $5.32 \pm 0.05$ & $\ldots$ & $\begin{array}{c}12.00+1 \\
\ldots\end{array}$ & 2004 Oct 7 \\
\hline J04314444+1808315 & L1551NE & 30 & $8.39 \pm 0.05$ & $6.94 \pm 0.05$ & $\ldots$ & $\ldots$ & 2004 Oct 7 \\
\hline \multirow{3}{*}{$\mathrm{J} 04331650+2253204$} & IRAS $04302+2247$ & 30 & $9.51 \pm 0.05$ & $9.21 \pm 0.05$ & $\ldots$ & $\ldots$ & 2005 Feb 20 \\
\hline & & 30 & $9.52 \pm 0.05$ & out & $\ldots$ & $\ldots$ & 2005 Feb 24 \\
\hline & & 30 & $9.63 \pm 0.05$ & $9.31 \pm 0.05$ & $\ldots$ & $\ldots$ & 2007 Apr 3 \\
\hline \multirow[t]{3}{*}{ J04353539+2408194 } & IRAS $04325+2402 \mathrm{~A}+\mathrm{B}+\mathrm{C}$ & 30 & $8.98 \pm 0.05$ & $8.55 \pm 0.05$ & $8.49 \pm 0.05$ & $8.16 \pm 0.05$ & 2005 Feb 20 \\
\hline & & 30 & $9.00 \pm 0.05$ & $8.47 \pm 0.05$ & $8.45 \pm 0.05$ & $8.17 \pm 0.05$ & 2005 Feb 21 \\
\hline & & 30 & $9.07 \pm 0.05$ & $8.61 \pm 0.05$ & $8.56 \pm 0.05$ & $8.24 \pm 0.05$ & 2006 Mar 25 \\
\hline \multirow[t]{2}{*}{ J04391389+2553208 } & IRAS $04361+2547$ & 30 & $8.27 \pm 0.05$ & $7.40 \pm 0.05$ & $\ldots$ & $\ldots$ & 2004 Mar 7 \\
\hline & & 30 & $7.69 \pm 0.05$ & $6.80 \pm 0.05$ & & & 2005 Feb 22 \\
\hline \multirow[t]{2}{*}{$\cdots$} & IRAS $04368+2557$ & 70 & $8.35 \pm 0.2$ & $7.35 \pm 0.2$ & $7.16 \pm 0.2$ & $7.40 \pm 0.2$ & 2004 Mar 7 \\
\hline & & 70 & $8.41 \pm 0.2$ & $7.50 \pm 0.2$ & $7.32 \pm 0.2$ & $7.76 \pm 0.2$ & 2005 Feb 22 \\
\hline
\end{tabular}

Nоте. - Entries of "out" indicate measurements that are absent because of a position outside the field of view of the camera. We have not measured photometry with a large aperture if a source does not exhibit significant extended emission in a given band $(\cdots)$. Meausrements with the default 4-pixel apertures are available in Table 4

a 2MASS Point Source Catalog.

b Aperture radius in pixels $(1$ pixel $=0$ ". 86$)$.

TABLE 6

MIPS $24 \mu \mathrm{M}$ Photometry For Members of TAurus

\begin{tabular}{clrl}
\hline \hline \multicolumn{1}{c}{ 2MASS $^{\mathrm{a}}$} & \multicolumn{1}{c}{ Name } & \multicolumn{1}{c}{$[24]$} & \multicolumn{1}{c}{ Date } \\
\hline J04034930+2610520 & HBC 358 A+B+C & $8.98 \pm 0.08$ & 2005 Feb 26 \\
& & $8.67 \pm 0.13$ & 2005 Mar 6 \\
J04034997+2620382 & XEST 06-006 & out & $\ldots$ \\
J04035084+2610531 & HBC 359 & $9.23 \pm 0.08$ & 2005 Feb 26 \\
J04043936+2158186 & HBC 360 & out & $\ldots$ \\
J04043984+2158215 & HBC 361 & out & $\ldots$ \\
J04044307+2618563 & IRAS 04016+2610 & sat & $\ldots$ \\
J04053087+2151106 & HBC 362 & $9.40 \pm 0.06$ & 2004 Sep 25 \\
J04080782+2807280 & $\ldots$ & out & $\ldots$ \\
J04131414+2819108 & LkCa 1 & $8.13 \pm 0.07$ & 2005 Feb 26 \\
& & $8.21 \pm 0.07$ & 2005 Mar 1 \\
& & $8.07 \pm 0.05$ & 2007 Feb 26 \\
J04132722+2816247 & Anon 1 & $7.28 \pm 0.07$ & 2005 Feb 26 \\
& & $6.90 \pm 0.04$ & 2005 Mar 1 \\
& & $6.94 \pm 0.04$ & 2007 Feb 26 \\
J04135328+2811233 & IRAS 04108+2803 A & $3.54 \pm 0.04$ & 2005 Mar 1 \\
& & $3.46 \pm 0.04$ & 2007 Feb 26 \\
J04135471+2811328 & IRAS 04108+2803 B & $1.36 \pm 0.04$ & 2005 Mar 1 \\
& & $1.04 \pm 0.04$ & 2007 Feb 26 \\
J04135737+2918193 & IRAS 04108+2910 & $3.10 \pm 0.04$ & 2007 Feb 26 \\
J04141188+2811535 & $\ldots$ & $5.80 \pm 0.06$ & 2005 Mar 1 \\
& & $5.83 \pm 0.05$ & 2007 Feb 26 \\
$\ldots$ & IRAS 04111+2800G & $3.48 \pm 0.04$ & 2005 Mar 1 \\
& & $3.45 \pm 0.04$ & 2007 Feb 26 \\
J04141291+2812124 & V773 Tau A+B & $1.43 \pm 0.04$ & 2005 Mar 1 \\
\hline
\end{tabular}


TABLE 6 - Continued

\begin{tabular}{|c|c|c|c|}
\hline $2 \mathrm{MASS}^{\mathrm{a}}$ & Name & {$[24]$} & Date \\
\hline \multirow{3}{*}{ J04141358+2812492 } & \multirow{3}{*}{ FM Tau } & $1.56 \pm 0.04$ & 2007 Feb 26 \\
\hline & & $2.79 \pm 0.04$ & 2005 Mar 1 \\
\hline & & $2.91 \pm 0.04$ & 2007 Feb 26 \\
\hline \multirow[t]{2}{*}{ J04141458+2827580 } & \multirow[t]{2}{*}{ FN Tau } & $1.86 \pm 0.04$ & 2005 Mar 1 \\
\hline & & $2.00 \pm 0.04$ & 2007 Feb 26 \\
\hline \multirow[t]{2}{*}{ J04141700+2810578 } & \multirow[t]{2}{*}{ CW Tau } & $1.51 \pm 0.04$ & 2005 Mar 1 \\
\hline & & $1.61 \pm 0.04$ & 2007 Feb 26 \\
\hline \multirow{2}{*}{ J04141760+2806096 } & \multirow[t]{2}{*}{ CIDA 1} & $3.53 \pm 0.04$ & 2005 Mar 1 \\
\hline & & $3.50 \pm 0.04$ & 2007 Feb 26 \\
\hline J04142626+2806032 & MHO $1+$ MHO $2^{\mathrm{b}}$ & sat & $\cdots$ \\
\hline J04143054+2805147 & MHO 3 & sat & $\ldots$ \\
\hline J04144730+2646264 & FP Tau & $4.28 \pm 0.04$ & 2007 Feb 26 \\
\hline \multirow{2}{*}{ J04144739+2803055 } & XEST 20-066 & $9.14 \pm 0.10$ & 2005 Mar 1 \\
\hline & & $9.37 \pm 0.23$ & 2007 Feb 26 \\
\hline J04144786+2648110 & CX Tau & $3.38 \pm 0.04$ & 2007 Feb 26 \\
\hline J04144797+2752346 & LkCa $3 \mathrm{~A}+\mathrm{B}$ & $7.07 \pm 0.05$ & 2005 Feb 26 \\
\hline & & $7.17 \pm 0.04$ & 2005 Mar 1 \\
\hline & & $7.09 \pm 0.04$ & 2007 Feb 26 \\
\hline J04144928+2812305 & FO Tau $\mathrm{A}+\mathrm{B}$ & $2.77 \pm 0.04$ & 2005 Mar 1 \\
\hline & & $2.88 \pm 0.04$ & 2007 Feb 26 \\
\hline J04145234+2805598 & XEST 20-071 & $7.04 \pm 0.04$ & 2005 Mar 1 \\
\hline & & $7.15 \pm 0.05$ & 2007 Feb 26 \\
\hline J04150515+2808462 & CIDA 2 & $8.43 \pm 0.07$ & 2005 Mar 1 \\
\hline & & $8.50 \pm 0.10$ & 2007 Feb 26 \\
\hline J04151471+2800096 & KPNO 1 & $\ldots$ & $\ldots$ \\
\hline J04152409+2910434 & & & $\cdots$ \\
\hline J04153916+2818586 & $\cdots$ & $4.28 \pm 0.04$ & 2004 Sep 23 \\
\hline & & $4.20 \pm 0.04$ & 2007 Feb 23 \\
\hline J04154278+2909597 & IRAS $04125+2902$ & $4.50 \pm 0.04$ & 2007 Feb 23 \\
\hline J04155799+2746175 & $\ldots$ & $5.78 \pm 0.04$ & 2007 Feb 23 \\
\hline J04161210+2756385 & $\ldots$ & $5.44 \pm 0.04$ & 2004 Sep 23 \\
\hline & & $5.38 \pm 0.04$ & 2007 Feb 23 \\
\hline J04161885+2752155 & $\cdots$ & $\ldots$ & $\ldots$ \\
\hline J04162725+2053091 & & out & $\ldots$ \\
\hline J04162810+2807358 & LkCa 4 & $7.92 \pm 0.04$ & 2004 Sep 23 \\
\hline & & $7.96 \pm 0.06$ & 2007 Feb 23 \\
\hline J04163048+3037053 & $\ldots$ & out & \\
\hline J04163911+2858491 & & $7.25 \pm 0.05$ & 2007 Feb 23 \\
\hline J04173372+2820468 & CY Tau & $4.43 \pm 0.04$ & 2004 Sep 23 \\
\hline & & $4.44 \pm 0.04$ & 2005 Feb 28 \\
\hline & & $4.41 \pm 0.04$ & 2007 Feb 23 \\
\hline J04173893+2833005 & LkCa 5 & $8.44 \pm 0.07$ & 2004 Sep 19 \\
\hline & & $8.67 \pm 0.06$ & 2004 Sep 23 \\
\hline & & $8.60 \pm 0.08$ & 2007 Feb 23 \\
\hline J04174955+2813318 & KPNO 10 & $5.92 \pm 0.04$ & 2004 Sep 23 \\
\hline & & $5.99 \pm 0.04$ & 2005 Feb 28 \\
\hline & & $5.93 \pm 0.04$ & 2007 Feb 23 \\
\hline J04174965+2829362 & V410 X-ray 1 & $3.92 \pm 0.04$ & 2004 Sep 19 \\
\hline & & $3.96 \pm 0.04$ & 2004 Sep 23 \\
\hline & & $3.85 \pm 0.04$ & 2005 Feb 28 \\
\hline & & $3.81 \pm 0.04$ & 2007 Feb 23 \\
\hline J04180796+2826036 & V410 X-ray 3 & $9.19 \pm 0.06$ & 2004 Sep 23 \\
\hline & & $8.74 \pm 0.16$ & 2005 Feb 28 \\
\hline J04181078+2519574 & V409 Tau & $4.49 \pm 0.04$ & 2007 Feb 23 \\
\hline J04181710+2828419 & V410 Anon 13 & $6.07 \pm 0.04$ & 2004 Sep 23 \\
\hline & & $6.06 \pm 0.04$ & 2005 Feb 28 \\
\hline & & $6.03 \pm 0.04$ & 2007 Feb 23 \\
\hline J04182147+1658470 & HBC 372 & out & \\
\hline J04182909+2826191 & V410 Anon 25 & $8.18 \pm 0.05$ & 2004 Sep 23 \\
\hline & & $8.00 \pm 0.07$ & 2005 Feb 28 \\
\hline & & $8.18 \pm 0.09$ & 2007 Feb 23 \\
\hline $\mathrm{J} 04183030+2743208$ & KPNO 11 & & $\ldots$ \\
\hline J04183110+2827162 & V410 Tau A+B+C & $7.13 \pm 0.04$ & 2004 Sep 23 \\
\hline & & $7.12 \pm 0.05$ & 2005 Feb 28 \\
\hline & & $7.11 \pm 0.05$ & 2007 Feb 23 \\
\hline J04183112+2816290 & DD Tau A+B & $1.73 \pm 0.04$ & 2004 Sep 23 \\
\hline & & $1.67 \pm 0.04$ & 2005 Feb 28 \\
\hline J04183158+2816585 & CZ Tau A+B & $1.96 \pm 0.04$ & 2004 Sep 23 \\
\hline & & $1.94 \pm 0.04$ & 2005 Feb 28 \\
\hline J04183203+2831153 & IRAS $04154+2823$ & $1.90 \pm 0.04$ & 2004 Sep 23 \\
\hline & & $2.06 \pm 0.04$ & 2005 Feb 28 \\
\hline & & $1.82 \pm 0.04$ & 2007 Feb 23 \\
\hline J04183444+2830302 & V410 X-ray 2 & $3.42 \pm 0.04$ & 2004 Sep 23 \\
\hline & & $3.48 \pm 0.04$ & 2005 Feb 28 \\
\hline & & $3.34 \pm 0.04$ & 2007 Feb 23 \\
\hline J04184023+2824245 & V410 X-ray 4 & $8.07 \pm 0.06$ & 2004 Sep 23 \\
\hline
\end{tabular}


TABLE 6 - Continued

\begin{tabular}{|c|c|c|c|}
\hline $2 \mathrm{MASS}^{\mathrm{a}}$ & Name & {$[24]$} & Date \\
\hline & & $\begin{array}{l}7.90 \pm 0.08 \\
8.20 \pm 0.10\end{array}$ & $\begin{array}{l}2005 \text { Feb } 28 \\
2007 \text { Feb } 23\end{array}$ \\
\hline J04184061+2819155 & V892 Tau & sat & ... \\
\hline \multirow[t]{3}{*}{ J04184133+2827250 } & LR1 & $4.61 \pm 0.04$ & 2004 Sep 23 \\
\hline & & $4.63 \pm 0.04$ & 2005 Feb 28 \\
\hline & & $4.64 \pm 0.04$ & 2007 Feb 23 \\
\hline J04184250+2818498 & V410 X-ray 7 & & \\
\hline \multirow[t]{3}{*}{$\mathrm{J} 04184703+2820073$} & Hubble 4 & $6.99 \pm 0.23$ & 2004 Sep 23 \\
\hline & & $6.62 \pm 0.10$ & 2005 Feb 28 \\
\hline & & $6.60 \pm 0.15$ & $2007 \mathrm{Feb} 23$ \\
\hline J04185115+2814332 & KPNO 2 & & $\ldots$ \\
\hline \multirow{3}{*}{$\mathrm{J} 04185147+2820264$} & $\mathrm{CoKu} \mathrm{Tau} / 1$ & $0.83 \pm 0.04$ & 2004 Sep 23 \\
\hline & & $0.85 \pm 0.04$ & 2005 Feb 28 \\
\hline & & $0.84 \pm 0.04$ & $2007 \mathrm{Feb} 23$ \\
\hline J04185170+1723165 & HBC 376 & $8.95 \pm 0.08$ & 2004 Sep 25 \\
\hline \multirow[t]{3}{*}{ J04185813+2812234 } & IRAS $04158+2805$ & $2.71 \pm 0.04$ & 2004 Sep 23 \\
\hline & & $2.74 \pm 0.04$ & $2005 \mathrm{Feb} 28$ \\
\hline & & $2.73 \pm 0.04$ & $2007 \mathrm{Feb} 23$ \\
\hline \multirow{3}{*}{ J04190110+2819420 } & V410 X-ray 6 & $3.63 \pm 0.04$ & 2004 Sep 23 \\
\hline & & $3.62 \pm 0.04$ & 2005 Feb 28 \\
\hline & & $3.69 \pm 0.04$ & $2007 \mathrm{Feb} 23$ \\
\hline J04190126+2802487 & KPNO 12 & & \\
\hline \multirow[t]{3}{*}{ J04190197+2822332 } & V410 X-ray 5a & $8.84 \pm 0.07$ & 2004 Sep 23 \\
\hline & & $9.09 \pm 0.17$ & 2005 Feb 28 \\
\hline & & $9.08 \pm 0.24$ & 2007 Feb 23 \\
\hline \multirow[t]{3}{*}{$\mathrm{J} 04191281+2829330$} & FQ Tau A+B & $4.92 \pm 0.04$ & 2004 Sep 23 \\
\hline & & $4.84 \pm 0.04$ & 2005 Feb 28 \\
\hline & & $4.82 \pm 0.04$ & 2007 Feb 23 \\
\hline J04191583+2906269 & BP Tau & $2.51 \pm 0.04$ & 2007 Feb 23 \\
\hline \multirow{3}{*}{$\mathrm{J} 04192625+2826142$} & V819 Tau & $6.33 \pm 0.04$ & 2004 Sep 23 \\
\hline & & $6.30 \pm 0.04$ & 2005 Feb 28 \\
\hline & & $6.36 \pm 0.04$ & 2007 Feb 23 \\
\hline \multirow[t]{3}{*}{ J04193545+2827218 } & FR Tau & $4.79 \pm 0.04$ & 2004 Sep 23 \\
\hline & & $4.84 \pm 0.04$ & 2005 Feb 28 \\
\hline & & $4.82 \pm 0.04$ & 2007 Feb 23 \\
\hline J04194127+2749484 & $\mathrm{LkCa} 7 \mathrm{~A}+\mathrm{B}$ & $7.74 \pm 0.05$ & 2005 Feb 26 \\
\hline & & $7.71 \pm 0.06$ & 2005 Feb 28 \\
\hline & & $7.79 \pm 0.06$ & 2007 Oct 28 \\
\hline J04194148+2716070 & IRAS $04166+2708$ & $3.19 \pm 0.04$ & 2005 Feb 28 \\
\hline & & $3.25 \pm 0.04$ & 2007 Oct 28 \\
\hline & IRAS $04166+2706$ & $2.91 \pm 0.04$ & 2005 Feb 28 \\
\hline & & $2.90 \pm 0.04$ & 2007 Oct 28 \\
\hline J04194657+2712552 & [GKH94] 41 & $4.07 \pm 0.04$ & 2005 Feb 28 \\
\hline & & $4.07 \pm 0.04$ & 2007 Oct 28 \\
\hline J04195844+2709570 & IRAS $04169+2702$ & sat & $\ldots$ \\
\hline J04201611+2821325 & $\ldots$ & $8.16 \pm 0.06$ & 2005 Feb 28 \\
\hline & & $8.20 \pm 0.09$ & 2007 Feb 23 \\
\hline J04202144+2813491 & $\ldots$ & $8.63 \pm 0.15$ & $2005 \mathrm{Feb} 28$ \\
\hline & & $8.46 \pm 0.13$ & $2007 \mathrm{Feb} 23$ \\
\hline & & $8.68 \pm 0.09$ & 2007 Oct 29 \\
\hline J04202555+2700355 & $\cdots$ & $6.11 \pm 0.04$ & 2005 Feb 28 \\
\hline & & $6.07 \pm 0.04$ & 2007 Oct 28 \\
\hline J04202583+2819237 & IRAS $04173+2812$ & $3.73 \pm 0.04$ & 2005 Feb 28 \\
\hline & & $3.83 \pm 0.04$ & 2007 Feb 23 \\
\hline J04202606+2804089 & $\ldots$ & $3.25 \pm 0.04$ & 2005 Feb 28 \\
\hline J04203918+2717317 & XEST 16-045 & $9.06 \pm 0.13$ & 2005 Feb 28 \\
\hline & & $8.91 \pm 0.11$ & 2007 Oct 28 \\
\hline J04205273+1746415 & $\mathrm{J} 2-157$ & & $\ldots$ \\
\hline J04210795+2702204 & & $1.69 \pm 0.04$ & 2005 Feb 28 \\
\hline & & $1.50 \pm 0.04$ & 2007 Oct 28 \\
\hline J04210934+2750368 & $\ldots$ & $7.13 \pm 0.05$ & 2005 Feb 28 \\
\hline & & $7.17 \pm 0.05$ & 2007 Oct 28 \\
\hline J04211038+2701372 & IRAS $04181+2654$ B & $2.64 \pm 0.04$ & 2005 Feb 28 \\
\hline & & $2.67 \pm 0.04$ & 2007 Oct 28 \\
\hline J04211146+2701094 & IRAS $04181+2654 \mathrm{~A}$ & $1.65 \pm 0.04$ & 2005 Feb 28 \\
\hline & & $1.52 \pm 0.04$ & 2007 Oct 28 \\
\hline J04213459+2701388 & $\ldots$ & $7.18 \pm 0.05$ & 2005 Feb 28 \\
\hline & & $7.02 \pm 0.05$ & $2007 \mathrm{Feb} 28$ \\
\hline & & $7.19 \pm 0.05$ & 2007 Oct 28 \\
\hline J04214013+2814224 & XEST 21-026 & $\ldots$ & $\cdots$ \\
\hline J04214323+1934133 & IRAS $04187+1927$ & out & $\ldots$ \\
\hline J04214631+2659296 & & $7.21 \pm 0.05$ & 2005 Feb 28 \\
\hline & & $7.42 \pm 0.05$ & 2007 Feb 28 \\
\hline & & $7.23 \pm 0.05$ & 2007 Oct 28 \\
\hline J04215450+2652315 & $\ldots$ & & $\ldots$ \\
\hline J04215563+2755060 & DE Tau & $2.56 \pm 0.04$ & 2005 Feb 27 \\
\hline & & $2.58 \pm 0.04$ & 2007 Oct 28 \\
\hline
\end{tabular}


Disk Population of Taurus

TABLE 6 - Continued

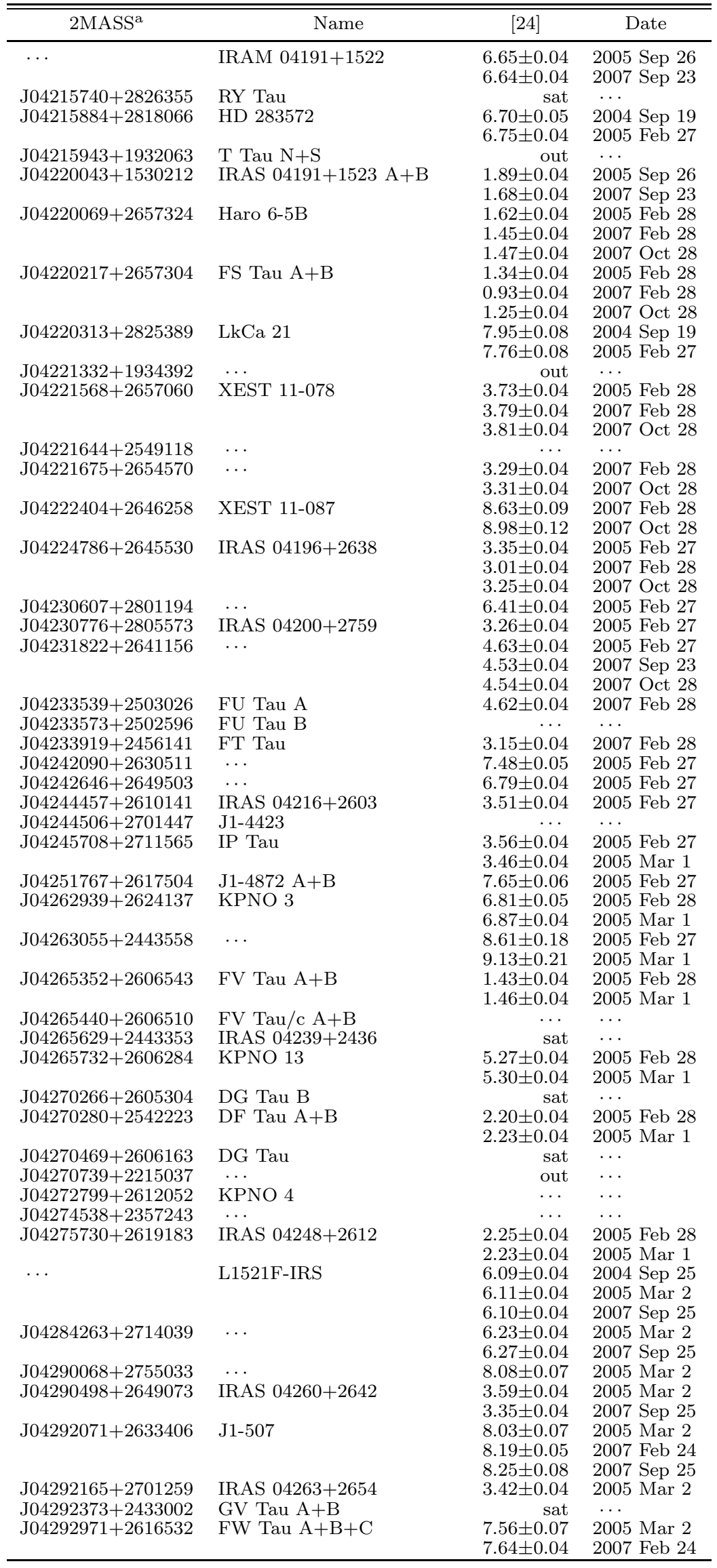


Luhman et al.

TABLE 6 - Continued

\begin{tabular}{|c|c|c|c|}
\hline $2 \mathrm{MASS}^{\mathrm{a}}$ & Name & [24] & Date \\
\hline J04293008+2439550 & IRAS $04264+2433$ & $\begin{array}{l}0.97 \pm 0.04 \\
1.09 \pm 0.04\end{array}$ & $\begin{array}{l}2005 \text { Mar } 2 \\
2007 \text { Sep } 23\end{array}$ \\
\hline J04293209+2430597 & $\cdots$ & $3.90 \pm 0.04$ & 2005 Mar 2 \\
\hline J04293606+2435556 & XEST 13-010 & $\begin{array}{l}4.15 \pm 0.04 \\
4.11 \pm 0.04\end{array}$ & 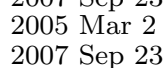 \\
\hline J04294155+2632582 & DH Tau A+B & $\begin{array}{l}3.32 \pm 0.04 \\
3.34 \pm 0.04 \\
3.21 \pm 0.04\end{array}$ & $\begin{array}{l}2004 \text { Sep } 25 \\
2005 \text { Mar } 2 \\
2007 \text { Feb } 24\end{array}$ \\
\hline $\begin{array}{l}\text { J04294247+2632493 } \\
\text { J04294568+2630468 }\end{array}$ & $\begin{array}{l}\text { DI Tau A+B } \\
\text { KPNO } 5\end{array}$ & & \\
\hline J04295156+2606448 & IQ Tau & $\begin{array}{l}2.81 \pm 0.04 \\
2.84 \pm 0.04\end{array}$ & $\begin{array}{l}2005 \text { Mar } 2 \\
2007 \text { Feb } 24\end{array}$ \\
\hline J04295422+1754041 & $\cdots$ & out & \\
\hline J04295950+2433078 & $\cdots$ & $\begin{array}{l}4.92 \pm 0.04 \\
4.86 \pm 0.04\end{array}$ & $\begin{array}{l}2005 \text { Mar } 2 \\
2007 \text { Sep } 23\end{array}$ \\
\hline J04300399+1813493 & UX Tau $\mathrm{A}+\mathrm{B}+\mathrm{C}$ & $1.83 \pm 0.04$ & 2004 Sep 25 \\
\hline J04300724+2608207 & KPNO 6 & $\begin{array}{l}9.10 \pm 0.22 \\
9.20 \pm 0.09\end{array}$ & $\begin{array}{l}2005 \text { Mar } 2 \\
2007 \text { Feb } 24\end{array}$ \\
\hline J04302365+2359129 & & & \\
\hline J04302961+2426450 & FX Tau A+B & $\begin{array}{l}3.04 \pm 0.04 \\
3.04 \pm 0.04 \\
2.88 \pm 0.04\end{array}$ & $\begin{array}{l}2005 \text { Feb } 26 \\
2005 \text { Mar } 2 \\
2007 \text { Sep } 23\end{array}$ \\
\hline $\begin{array}{l}\text { J04304425+2601244 } \\
\text { J04305028+2300088 }\end{array}$ & $\begin{array}{l}\text { DK Tau A+B } \\
\text { IRAS } 04278+2253 \mathrm{~A}+\mathrm{B}\end{array}$ & $\begin{array}{r}1.84 \pm 0.04 \\
\text { sat }\end{array}$ & 2005 Mar 2 \\
\hline J04305137+2442222 & ZZ Tau & $\begin{array}{l}4.42 \pm 0.04 \\
4.54 \pm 0.04\end{array}$ & $\begin{array}{l}2004 \text { Sep } 25 \\
2005 \text { Mar } 2\end{array}$ \\
\hline $\mathrm{J} 04305171+2441475$ & ZZ Tau IRS & $\begin{array}{l}2.06 \pm 0.04 \\
2.02 \pm 0.04\end{array}$ & $\begin{array}{l}2004 \text { Sep } 25 \\
2005 \text { Mar } 2\end{array}$ \\
\hline J04305718+2556394 & KPNO 7 & $8.44 \pm 0.09$ & 2005 Mar 2 \\
\hline J04311444+2710179 & JH 56 & $6.72 \pm 0.04$ & 2005 Mar 1 \\
\hline $\begin{array}{l}\text { J04311578+1820072 } \\
\text { J04311907+2335047 }\end{array}$ & $\begin{array}{l}\text { MHO } 9 \\
\ldots\end{array}$ & $9.80 \pm 0.15$ & $\begin{array}{c}2006 \text { Feb } 19 \\
\ldots\end{array}$ \\
\hline $\mathrm{J} 04312382+2410529$ & V927 Tau A+B & $\begin{array}{l}8.25 \pm 0.07 \\
8.20 \pm 0.08\end{array}$ & $\begin{array}{l}2004 \text { Sep } 25 \\
2005 \text { Mar } 2\end{array}$ \\
\hline $\begin{array}{l}\text { J04312405+1800215 } \\
\text { J04312669+2703188 }\end{array}$ & MHO 4 & $\begin{array}{c}9.62 \pm 0.12 \\
\ldots\end{array}$ & $\begin{array}{c}2006 \text { Feb } 19 \\
\ldots\end{array}$ \\
\hline J04313407+1808049 & L1551/IRS5 & sat & $\ldots$ \\
\hline J04313613+1813432 & LkHa 358 & $\begin{array}{l}2.48 \pm 0.04 \\
2.64 \pm 0.05\end{array}$ & $\begin{array}{l}2004 \text { Feb } 20 \\
2006 \text { Feb } 19\end{array}$ \\
\hline J04313747+1812244 & HH 30 & $\begin{array}{l}6.84 \pm 0.06 \\
6.40 \pm 0.04\end{array}$ & $\begin{array}{l}2004 \text { Feb } 20 \\
2006 \text { Feb } 19\end{array}$ \\
\hline $\begin{array}{l}\text { J04313843+1813576 } \\
\text { J04314007+1813571 }\end{array}$ & $\begin{array}{l}\text { HL Tau } \\
\text { XZ Tau A+B }\end{array}$ & $\begin{array}{l}\text { sat } \\
\text { sat }\end{array}$ & $\begin{array}{l}\cdots \\
\cdots\end{array}$ \\
\hline J04314444+1808315 & L1551NE & $0.55 \pm 0.04$ & 2006 Feb 19 \\
\hline J04315056+2424180 & HK Tau A+B & $\begin{array}{l}2.35 \pm 0.04 \\
2.31 \pm 0.04\end{array}$ & $\begin{array}{l}2004 \text { Sep } 25 \\
2005 \text { Mar } 2\end{array}$ \\
\hline & & $2.28 \pm 0.04$ & 2007 Sep 23 \\
\hline J04315779+1821380 & V710 Tau A+B & $\begin{array}{l}3.65 \pm 0.04 \\
3.64 \pm 0.04\end{array}$ & $\begin{array}{l}2004 \text { Feb } 20 \\
2004 \text { Sep } 25\end{array}$ \\
\hline & & $3.67 \pm 0.04$ & $2006 \mathrm{Feb} 19$ \\
\hline J04315844+2543299 & $\mathrm{J} 1-665$ & $9.26 \pm 0.18$ & 2005 Mar 1 \\
\hline J04315968+1821305 & LkHa 267 & $\begin{array}{l}4.51 \pm 0.04 \\
4.55 \pm 0.04\end{array}$ & $\begin{array}{l}2004 \text { Feb } 20 \\
2004 \text { Sep } 25\end{array}$ \\
\hline & & $4.50 \pm 0.04$ & 2006 Feb 19 \\
\hline J04320329+2528078 & & & \\
\hline J04320926+1757227 & L1551-51 & $\begin{array}{l}8.81 \pm 0.07 \\
8.56 \pm 0.08 \\
8.49 \pm 0.06\end{array}$ & $\begin{array}{l}2004 \text { Feb } 20 \\
2004 \text { Sep } 23 \\
2006 \text { Feb } 19\end{array}$ \\
\hline J04321456+1820147 & V827 Tau & $\begin{array}{l}7.95 \pm 0.06 \\
7.81 \pm 0.05\end{array}$ & $\begin{array}{l}2004 \text { Feb } 20 \\
2006 \text { Feb } 19\end{array}$ \\
\hline J04321540+2428597 & Haro 6-13 & sat & \\
\hline J04321583+1801387 & V826 Tau A+B & $\begin{array}{l}7.78 \pm 0.05 \\
7.88 \pm 0.04\end{array}$ & $\begin{array}{l}2004 \text { Feb } 20 \\
2006 \text { Feb } 19\end{array}$ \\
\hline J04321606+1812464 & MHO 5 & $\begin{array}{l}5.68 \pm 0.04 \\
5.64 \pm 0.04\end{array}$ & $\begin{array}{l}2004 \text { Feb } 20 \\
2006 \text { Feb } 19\end{array}$ \\
\hline J04321786+2422149 & & $9.69 \pm 0.10$ & 2007 Sep 23 \\
\hline $\mathrm{J} 04321885+2422271$ & V928 Tau A+B & $\begin{array}{l}7.59 \pm 0.05 \\
7.52 \pm 0.05\end{array}$ & $\begin{array}{l}2004 \text { Sep } 25 \\
2005 \text { Mar } 1\end{array}$ \\
\hline $\mathrm{J} 04322210+1827426$ & MHO 6 & $\begin{array}{l}7.58 \pm 0.04 \\
6.27 \pm 0.04 \\
6.43 \pm 0.04\end{array}$ & $\begin{array}{l}2007 \text { Sep } 23 \\
2004 \text { Feb } 20 \\
2006 \text { Feb } 19\end{array}$ \\
\hline J04322329+2403013 & $\cdots$ & & \\
\hline J04322415+2251083 & $\ldots$ & $6.12 \pm 0.04$ & 2005 Mar 2 \\
\hline J04322627+1827521 & MHO 7 & $9.82 \pm 0.24$ & 2006 Feb 19 \\
\hline J04323028+1731303 & GG Tau $\mathrm{Ba}+\mathrm{Bb}$ & out & $\ldots$ \\
\hline J04323034+1731406 & GG Tau $\mathrm{Aa}+\mathrm{Ab}$ & out & $\cdots$ \\
\hline
\end{tabular}


TABLE 6 - Continued

\begin{tabular}{|c|c|c|c|}
\hline $2 \mathrm{MASS}^{\mathrm{a}}$ & Name & {$[24]$} & Date \\
\hline \multirow[t]{2}{*}{ J04323058+2419572 } & FY Tau & $\begin{array}{l}3.79 \pm 0.04 \\
3.67 \pm 0.04\end{array}$ & $\begin{array}{l}2004 \text { Sep } 25 \\
2005 \text { Mar } 1\end{array}$ \\
\hline & & $3.75 \pm 0.04$ & 2007 Sep 23 \\
\hline \multirow[t]{2}{*}{ J04323176+2420029 } & FZ Tau & $1.78 \pm 0.04$ & 2004 Sep 25 \\
\hline & & $2.07 \pm 0.04$ & 2005 Mar 1 \\
\hline \multirow{2}{*}{ J04323205+2257266 } & IRAS $04295+2251$ & $\begin{array}{l}1.90 \pm 0.04 \\
1.25 \pm 0.04\end{array}$ & $\begin{array}{l}2007 \text { Sep } 23 \\
2005 \text { Mar } 2\end{array}$ \\
\hline & & $1.12 \pm 0.04$ & 2007 Feb 27 \\
\hline J04324303+2552311 & $\mathrm{UZ}$ Tau $\mathrm{A}+\mathrm{Ba}+\mathrm{Bb}$ & $1.57 \pm 0.04$ & 2005 Mar 1 \\
\hline \multirow{3}{*}{ J04324373+1802563 } & L1551-55 & $8.85 \pm 0.15$ & 2004 Feb 20 \\
\hline & & $9.11 \pm 0.10$ & 2004 Sep 25 \\
\hline & & $9.12 \pm 0.09$ & 2006 Feb 19 \\
\hline \multirow{2}{*}{ J04324911+2253027 } & JH 112 & $2.50 \pm 0.04$ & 2005 Mar 2 \\
\hline & & $2.54 \pm 0.04$ & 2007 Feb 26 \\
\hline J04324938+2253082 & $\cdots$ & & \\
\hline \multirow{3}{*}{$\mathrm{J} 04325026+2422115$} & $\ldots$ & $9.81 \pm 0.25$ & 2004 Sep 25 \\
\hline & & $9.40 \pm 0.07$ & 2005 Sep 25 \\
\hline & & $9.42 \pm 0.10$ & 2007 Sep 24 \\
\hline J04325119+1730092 & LH $0429+17$ & out & \\
\hline \multirow[t]{4}{*}{$\mathrm{J} 04330197+2421000$} & MHO 8 & $8.72 \pm 0.07$ & 2004 Sep 25 \\
\hline & & $9.24 \pm 0.15$ & 2005 Mar 1 \\
\hline & & $8.87 \pm 0.05$ & 2005 Sep 25 \\
\hline & & $8.83 \pm 0.09$ & 2007 Sep 24 \\
\hline \multirow{3}{*}{ J04330622+2409339 } & GH Tau A+B & $3.25 \pm 0.04$ & 2005 Feb 26 \\
\hline & & $3.19 \pm 0.04$ & 2005 Mar 1 \\
\hline & & $3.26 \pm 0.04$ & 2007 Sep 24 \\
\hline \multirow{3}{*}{ J04330664+2409549 } & V807 Tau A+B & $3.04 \pm 0.04$ & 2005 Feb 26 \\
\hline & & $2.95 \pm 0.04$ & 2005 Mar 1 \\
\hline & & $3.06 \pm 0.04$ & 2007 Sep 24 \\
\hline \multirow[t]{2}{*}{ J04330781+2616066 } & KPNO 14 & $9.27 \pm 0.25$ & 2005 Mar 1 \\
\hline & & $9.21 \pm 0.21$ & 2007 Oct 27 \\
\hline J04330945+2246487 & . & $8.25 \pm 0.08$ & 2005 Mar 1 \\
\hline & & $8.25 \pm 0.08$ & 2007 Feb 26 \\
\hline J04331003+2433433 & V830 Tau & $8.30 \pm 0.09$ & 2004 Sep 18 \\
\hline & & $8.25 \pm 0.08$ & $2005 \mathrm{Feb} 26$ \\
\hline & & $8.18 \pm 0.07$ & 2005 Mar 1 \\
\hline J04331435+2614235 & IRAS $04301+2608$ & $3.23 \pm 0.04$ & 2005 Mar 1 \\
\hline & & $3.35 \pm 0.04$ & 2007 Oct 27 \\
\hline J04331650+2253204 & IRAS $04302+2247$ & $3.56 \pm 0.04$ & 2005 Mar 1 \\
\hline & & $3.46 \pm 0.04$ & 2007 Feb 27 \\
\hline J04331907+2246342 & IRAS $04303+2240$ & $1.27 \pm 0.04$ & 2005 Mar 1 \\
\hline & & $1.44 \pm 0.04$ & 2007 Feb 26 \\
\hline J04332621+2245293 & XEST 17-036 & $8.34 \pm 0.09$ & 2005 Mar 1 \\
\hline & & $8.27 \pm 0.08$ & 2007 Feb 26 \\
\hline J04333278+1800436 & & out & \\
\hline J04333297+1801004 & HD $28867 \mathrm{~A}+\mathrm{B}+\mathrm{C}$ & out & $\ldots$ \\
\hline J04333405+2421170 & GI Tau & $1.93 \pm 0.04$ & 2004 Sep 18 \\
\hline & & $2.09 \pm 0.05$ & $2005 \mathrm{Feb} 26$ \\
\hline & & $2.05 \pm 0.04$ & 2005 Mar 1 \\
\hline J04333456+2421058 & GK Tau & $1.64 \pm 0.04$ & 2004 Sep 18 \\
\hline & & $1.69 \pm 0.04$ & 2005 Mar 1 \\
\hline J04333678+2609492 & IS Tau A+B & $3.69 \pm 0.04$ & 2005 Mar 1 \\
\hline J04333905+2227207 & & $4.89 \pm 0.04$ & 2007 Feb 26 \\
\hline $\mathrm{J} 04333906+2520382$ & DL Tau & $2.17 \pm 0.04$ & 2005 Mar 1 \\
\hline & & $2.12 \pm 0.04$ & 2007 Oct 27 \\
\hline J04333935+1751523 & HN Tau A+B & out & $\ldots$ \\
\hline J04334171+1750402 & & out & $\ldots$ \\
\hline $\mathrm{J} 04334291+2526470$ & $\cdots$ & & \\
\hline J04334465+2615005 & & $4.54 \pm 0.04$ & 2005 Mar 1 \\
\hline J04334871+1810099 & DM Tau & out & \\
\hline J04335200+2250301 & CI Tau & $2.12 \pm 0.04$ & 2005 Mar 1 \\
\hline J04335245+2612548 & $\ldots$ & $\begin{array}{l}2.15 \pm 0.04 \\
8.26 \pm 0.09\end{array}$ & $\begin{array}{l}2007 \text { Feb } 26 \\
2005 \text { Mar } 1\end{array}$ \\
\hline J04335252+2256269 & XEST 17-059 & $8.41 \pm 0.10$ & 2005 Mar 1 \\
\hline J04335470+2613275 & IT Tau A+B & $3.51 \pm 0.04$ & 2005 Mar 1 \\
\hline J04335546+1838390 & $\mathrm{J} 2-2041$ & out & $\ldots$ \\
\hline J04341099+2251445 & JH 108 & $8.75 \pm 0.12$ & 2005 Mar 1 \\
\hline & & $8.64 \pm 0.21$ & 2007 Feb 27 \\
\hline J04341527+2250309 & CFHT 1 & $\ldots$ & $\ldots$ \\
\hline J04341803+1830066 & НBC 407 & out & $\ldots$ \\
\hline J04344544+2308027 & & & \\
\hline J04345542+2428531 & AA Tau & $2.80 \pm 0.04$ & 2005 Mar 1 \\
\hline & & $2.78 \pm 0.04$ & 2006 Feb 26 \\
\hline J04345693+2258358 & XEST 08-003 & $8.34 \pm 0.07$ & 2005 Mar 1 \\
\hline & & $8.31 \pm 0.08$ & $2007 \mathrm{Feb} 26$ \\
\hline J04350850+2311398 & $\ldots$ & & \\
\hline J04352020+2232146 & HO Tau & $4.90 \pm 0.04$ & 2007 Feb 26 \\
\hline
\end{tabular}


TABLE 6 - Continued

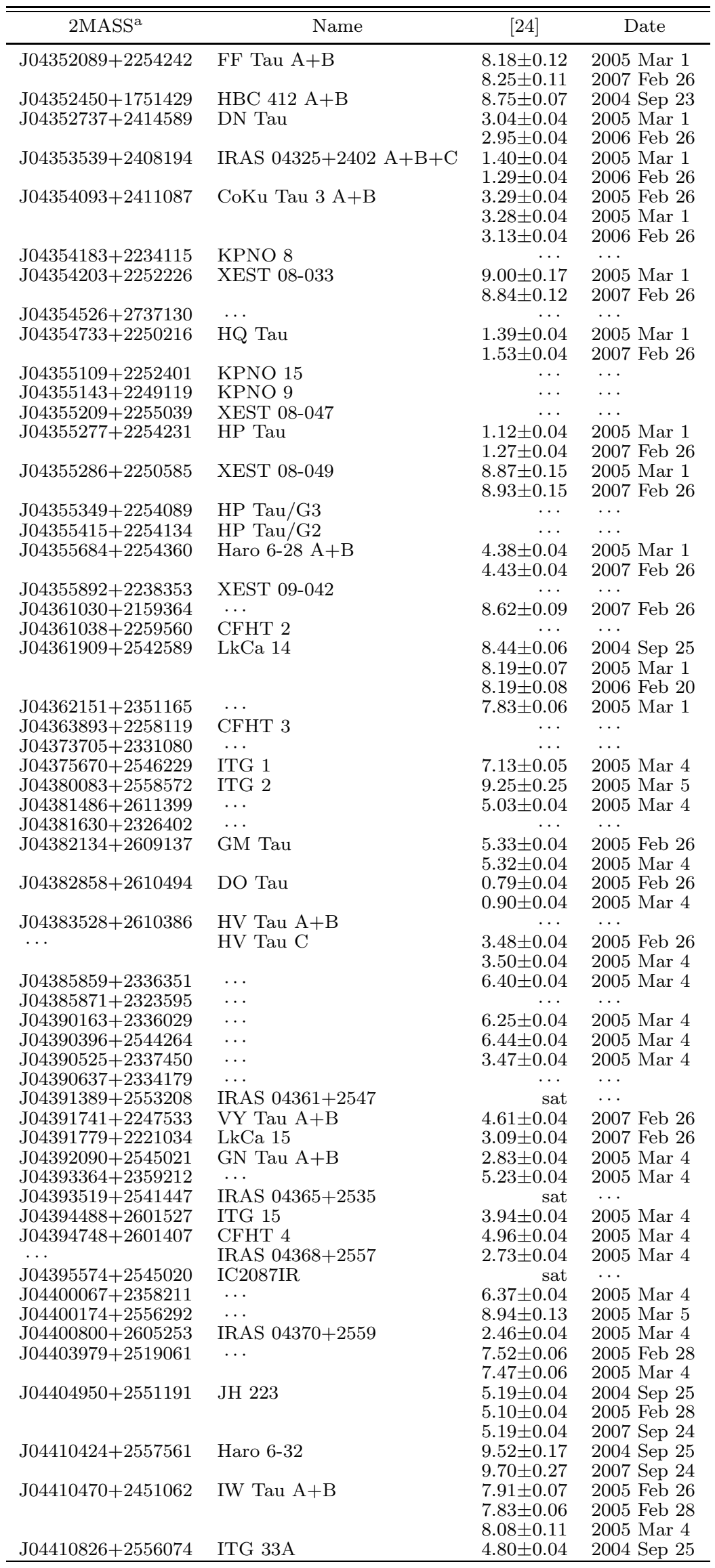


TABLE 6 - Continued

\begin{tabular}{|c|c|c|c|}
\hline $2 \mathrm{MASS}^{\mathrm{a}}$ & Name & {$[24]$} & Date \\
\hline & & $4.64 \pm 0.04$ & 2005 Feb 26 \\
\hline & & $4.65 \pm 0.04$ & $2005 \mathrm{Feb} 28$ \\
\hline & & $4.40 \pm 0.04$ & 2007 Sep 24 \\
\hline \multirow{4}{*}{ J04411078+2555116 } & ITG 34 & $6.53 \pm 0.04$ & 2004 Sep 25 \\
\hline & & $6.58 \pm 0.04$ & 2005 Feb 26 \\
\hline & & $6.46 \pm 0.04$ & 2005 Feb 28 \\
\hline & & $6.52 \pm 0.04$ & 2007 Sep 24 \\
\hline \multirow[t]{4}{*}{ J04411267+2546354 } & IRAS $04381+2540$ & $1.43 \pm 0.04$ & 2004 Sep 25 \\
\hline & & $1.37 \pm 0.04$ & 2005 Feb 26 \\
\hline & & $1.35 \pm 0.04$ & 2005 Feb 28 \\
\hline & & $1.33 \pm 0.04$ & 2007 Sep 24 \\
\hline $\mathrm{J} 04411681+2840000$ & $\mathrm{CoKu} \mathrm{Tau} / 4$ & $2.30 \pm 0.04$ & 2007 Sep 25 \\
\hline \multirow[t]{4}{*}{ J04412464+2543530 } & ITG 40 & $5.54 \pm 0.04$ & 2004 Sep 25 \\
\hline & & $5.70 \pm 0.04$ & 2005 Feb 26 \\
\hline & & $5.65 \pm 0.04$ & 2005 Feb 28 \\
\hline & & $5.52 \pm 0.04$ & 2007 Sep 25 \\
\hline \multirow[t]{3}{*}{ J04413882+2556267 } & IRAS $04385+2550$ & $1.81 \pm 0.04$ & 2004 Sep 25 \\
\hline & & $1.84 \pm 0.04$ & 2005 Feb 28 \\
\hline & & $1.91 \pm 0.04$ & 2007 Sep 25 \\
\hline J04414489+2301513 & $\ldots$ & out & $\ldots$ \\
\hline J04414565+2301580 & $\ldots$ & out & $\ldots$ \\
\hline \multirow[t]{3}{*}{ J04414825+2534304 } & $\ldots$ & $6.26 \pm 0.04$ & 2004 Sep 25 \\
\hline & & $6.32 \pm 0.04$ & 2005 Feb 28 \\
\hline & & $6.31 \pm 0.04$ & 2007 Sep 25 \\
\hline \multirow{3}{*}{ J04420548+2522562 } & LkHa $332 / G 2 A+B$ & $7.26 \pm 0.07$ & 2004 Sep 25 \\
\hline & & $7.09 \pm 0.05$ & 2005 Feb 28 \\
\hline & & $7.24 \pm 0.09$ & 2007 Sep 25 \\
\hline J04420732+2523032 & LkHa $332 / \mathrm{G} 1 \mathrm{~A}+\mathrm{B}$ & & $\ldots$ \\
\hline \multirow{3}{*}{ J04420777+2523118 } & V955 Tau A+B & $2.86 \pm 0.04$ & 2004 Sep 25 \\
\hline & & $2.78 \pm 0.04$ & 2005 Feb 28 \\
\hline & & $2.73 \pm 0.04$ & 2007 Sep 25 \\
\hline \multirow{2}{*}{$\mathrm{J} 04422101+2520343$} & CIDA 7 & $4.22 \pm 0.04$ & 2004 Sep 25 \\
\hline & & $4.26 \pm 0.04$ & 2005 Feb 28 \\
\hline J04423769+2515374 & DP Tau & $1.85 \pm 0.04$ & 2005 Feb 28 \\
\hline J04430309+2520187 & GO Tau & $4.35 \pm 0.04$ & 2005 Feb 28 \\
\hline J04432023+2940060 & CIDA 14 & $5.56 \pm 0.04$ & 2004 Sep 23 \\
\hline J04442713+2512164 & IRAS $04414+2506$ & $4.30 \pm 0.04$ & 2005 Feb 28 \\
\hline J04455129+1555496 & HD 30171 & & \\
\hline J04455134+1555367 & IRAS $04429+1550$ & $2.55 \pm 0.04$ & 2005 Mar 3 \\
\hline J04464260+2459034 & RXJ $04467+2459$ & $\ldots$ & $\ldots$ \\
\hline J04465305+1700001 & DQ Tau & out & $\ldots$ \\
\hline J04465897+1702381 & Haro 6-37 A+B & out & $\cdots$ \\
\hline J04470620+1658428 & DR Tau & out & $\cdots$ \\
\hline J04474859+2925112 & DS Tau & out & $\ldots$ \\
\hline J04484189+1703374 & & out & $\ldots$ \\
\hline J04514737+3047134 & UY Aur A+B & out & $\ldots$ \\
\hline J04520668+3047175 & IRAS $04489+3042$ & out & $\ldots$ \\
\hline J04551098+3021595 & GM Aur & $2.48 \pm 0.04$ & 2005 Mar 5 \\
\hline J04552333+3027366 & & & \\
\hline J04553695+3017553 & LkCa 19 & $6.99 \pm 0.04$ & 2004 Oct 12 \\
\hline & & $7.09 \pm 0.04$ & 2005 Mar 5 \\
\hline J04554046+3039057 & $\cdots$ & & \\
\hline J04554535+3019389 & $\cdots$ & $6.41 \pm 0.04$ & 2004 Oct 12 \\
\hline & & $6.49 \pm 0.04$ & 2005 Mar 5 \\
\hline J04554582+3033043 & AB Aur & sat & $\ldots$ \\
\hline J04554757+3028077 & $\ldots$ & & $\ldots$ \\
\hline $\mathrm{J} 04554801+3028050$ & $\ldots$ & $7.05 \pm 0.04$ & 2005 Mar 5 \\
\hline J04554820+3030160 & XEST 26-052 & & \\
\hline J04554969+3019400 & $\ldots$ & $8.16 \pm 0.08$ & 2004 Sep 25 \\
\hline & & $7.94 \pm 0.07$ & 2004 Oct 12 \\
\hline & & $8.19 \pm 0.08$ & 2005 Mar 5 \\
\hline J04555288+3006523 & $\ldots$ & out & \\
\hline J04555605+3036209 & XEST 26-062 & $3.74 \pm 0.04$ & 2004 Sep 25 \\
\hline & & $3.77 \pm 0.04$ & 2005 Mar 5 \\
\hline J04555636+3049374 & $\ldots$ & $\ldots$ & $\ldots$ \\
\hline J04555938+3034015 & SU Aur & sat & $\ldots$ \\
\hline J04560118+3026348 & XEST26-071 & $5.81 \pm 0.04$ & 2005 Mar 5 \\
\hline $\mathrm{J} 04560201+3021037$ & HBC 427 & $7.67 \pm 0.08$ & 2004 Sep 25 \\
\hline & & $7.55 \pm 0.11$ & 2005 Mar 5 \\
\hline J04574903+3015195 & $\ldots$ & out & $\ldots$ \\
\hline J04584626+2950370 & MWC 480 & out & $\ldots$ \\
\hline J05030659+2523197 & V836 Tau & $3.83 \pm 0.04$ & 2004 Sep 25 \\
\hline J05044139+2509544 & CIDA 8 & $5.18 \pm 0.04$ & 2008 Apr 17 \\
\hline J05052286+2531312 & CIDA 9 & out & $\ldots$ \\
\hline J05061674+2446102 & CIDA 10 & out & $\ldots$ \\
\hline J05062332+2432199 & CIDA 11 & out & $\ldots$ \\
\hline J05064662+2104296 & 年 & out & $\ldots$ \\
\hline
\end{tabular}


TABLE 6 - Continued

\begin{tabular}{clll}
\hline \hline 2MASS & \multicolumn{1}{c}{ Name } & \multicolumn{1}{c}{ N24] } & \multicolumn{2}{c}{ Date } \\
\hline J05071206+2437163 & RXJ 05072+2437 & out & $\ldots$ \\
J05074953+3024050 & RW Aur A+B & out & $\ldots$ \\
J05075496+2500156 & CIDA 12 & out & $\ldots$
\end{tabular}

Note. - Entries of "...", "sat", and "out" indicate measurements that are absent because of non-detection, saturation, and a position outside the field of view of the camera, respectively.

a 2MASS Point Source Catalog.

b 2MASS J04142639+2805597.

TABLE 7

Spectral Slopes for Members of Taurus

\begin{tabular}{|c|c|c|c|c|c|c|c|}
\hline $2 \mathrm{MASS}^{\mathrm{a}}$ & Name & $\begin{array}{l}\text { Spectral } \\
\text { Type }\end{array}$ & $\alpha(2-8 \mu \mathrm{m})$ & $\alpha(2-24 \mu \mathrm{m})$ & $\alpha(3.6-8 \mu \mathrm{m})$ & $\alpha(3.6-24 \mu \mathrm{m})$ & $\begin{array}{l}\text { SED } \\
\text { Class }\end{array}$ \\
\hline J04034930+2610520 & $\mathrm{HBC} 358 \mathrm{~A}+\mathrm{B}+\mathrm{C}$ & M3.5 & $-2.49 /-2.49$ & $-2.68 /-2.68$ & $-2.74 /-2.74$ & $-2.83 /-2.83$ & III \\
\hline J04034997+2620382 & XEST 06-006 & M5.25 & & & & & III? \\
\hline J04035084+2610531 & HBC 359 & M2 & $-2.58 /-2.58$ & $-2.77 /-2.77$ & $-2.74 /-2.74$ & $-2.88 /-2.88$ & III \\
\hline J04043936+2158186 & HBC 360 & M3.5 & $-2.54 /-2.55$ & $\ldots$ & $-2.75 /-2.75$ & $\ldots$ & III \\
\hline J04043984+2158215 & HBC 361 & M3 & $-2.55 /-2.55$ & $\ldots$ & $-2.73 /-2.73$ & $\ldots$ & III \\
\hline J04044307+2618563 & IRAS $04016+2610$ & K3 & 1.74 & $\ldots$ & 0.85 & $\ldots$ & I \\
\hline J04053087+2151106 & HBC 362 & M2 & $-2.58 /-2.58$ & $-2.63 /-2.63$ & $-2.77 /-2.77$ & $-2.73 /-2.73$ & III \\
\hline J04080782+2807280 & & M3.75 & & & & & III? \\
\hline J04131414+2819108 & $\mathrm{LkCa} 1$ & M4 & $-2.64 /-2.71$ & $-2.69 /-2.73$ & $-2.76 /-2.79$ & $-2.76 /-2.77$ & III \\
\hline J04132722+2816247 & Anon 1 & M0 & $-2.51 /-2.65$ & $-2.71 /-2.79$ & $-2.69 /-2.76$ & $-2.84 /-2.87$ & III \\
\hline J04135328+2811233 & IRAS $04108+2803 \mathrm{~A}$ & M3.5-M6 & -0.09 & -0.23 & -0.13 & -0.28 & I \\
\hline J04135471+2811328 & IRAS $04108+2803 \mathrm{~B}$ & $\mathrm{~K} 6-\mathrm{M} 3.5^{\mathrm{c}}$ & 0.96 & 0.91 & 1.14 & 0.97 & I \\
\hline J04135737+2918193 & IRAS $04108+2910$ & M0 & $-0.23 /-0.43$ & $-0.47 /-0.57$ & $-0.61 /-0.70$ & $-0.68 /-0.72$ & II \\
\hline \multirow[t]{2}{*}{ J04141188+2811535 } & & M6.25 & $-0.85 /-0.94$ & $-0.63 /-0.68$ & $-0.55 /-0.60$ & $-0.45 /-0.47$ & II \\
\hline & IRAS $04111+2800 \mathrm{G}$ & $\mathrm{K} 6-\mathrm{M} 3.5^{\mathrm{c}}$ & & & 0.22 & 1.74 & I \\
\hline J04141291+2812124 & V773 Tau A+B & K3 & $-1.48 /-1.62$ & $-1.06 /-1.14$ & $-0.99 /-1.05$ & $-0.75 /-0.78$ & II \\
\hline J04141358+2812492 & FM Tau & M0 & $-1.03 /-1.13$ & $-0.60 /-0.65$ & $-0.92 /-0.97$ & $-0.44 /-0.46$ & II \\
\hline J04141458+2827580 & FN Tau & M5 & $-1.03 /-1.11$ & $-0.47 /-0.51$ & $-0.81 /-0.84$ & $-0.23 /-0.25$ & II \\
\hline $\mathrm{J} 04141700+2810578$ & CW Tau & K3 & $-0.80 /-1.01$ & $-0.73 /-0.84$ & $-1.11 /-1.21$ & $-0.85 /-0.89$ & II \\
\hline J04141760+2806096 & CIDA 1 & M5.5 & $-0.40 /-0.62$ & $-0.43 /-0.55$ & $-0.52 /-0.62$ & $-0.48 /-0.53$ & II \\
\hline J04142626+2806032 & MHO 1 & $\mathrm{M} 2.5$ & $-0.31 /-1.09$ & $\ldots$ & $-0.41 /-0.77$ & $\ldots$ & II \\
\hline J04142639+2805597 & MHO 2 & $\mathrm{M} 2.5$ & $-0.79 /-1.35$ & $\ldots$ & $-0.10 /-0.37$ & $\ldots$ & II \\
\hline $\mathrm{J} 04143054+2805147$ & MHO 3 & K7 & $-0.41 /-0.92$ & $\ldots$ & $-0.10 /-0.34$ & & II \\
\hline J04144730+2646264 & FP Tau & M4 & $-1.63 /-1.72$ & $-1.11 /-1.15$ & $-1.63 /-1.66$ & $-0.97 /-0.99$ & II \\
\hline J04144739+2803055 & XEST 20-066 & M5.25 & $-2.50 /-2.50$ & $-2.60 /-2.60$ & $-2.64 /-2.64$ & $-2.68 /-2.68$ & III \\
\hline J04144786+2648110 & CX Tau & M2.5 & $-1.22 /-1.26$ & $-0.79 /-0.81$ & $-0.73 /-0.75$ & $-0.47 /-0.48$ & II \\
\hline $\mathrm{J} 04144797+2752346$ & $\mathrm{LkCa} 3 \mathrm{~A}+\mathrm{B}$ & M1 & $-2.65 /-2.68$ & $-2.77 /-2.78$ & $-2.76 /-2.77$ & $-2.84 /-2.85$ & III \\
\hline J04144928+2812305 & FO Tau A+B & M3.5 & $-1.16 /-1.31$ & $-0.84 /-0.91$ & $-0.87 /-0.93$ & $-0.63 /-0.66$ & II \\
\hline J04145234+2805598 & XEST 20-071 & M3.25 & $-2.44 /-2.56$ & $-2.65 /-2.71$ & $-2.64 /-2.70$ & $-2.78 /-2.81$ & III \\
\hline J04150515+2808462 & CIDA 2 & M5.5 & $-2.52 /-2.56$ & $-2.64 /-2.66$ & $-2.72 /-2.74$ & $-2.76 /-2.76$ & III \\
\hline J04151471+2800096 & KPNO 1 & M8.5 & $-2.19 /-2.21$ & . & $-2.55 /-2.56$ & & III \\
\hline J04152409+2910434 & $\ldots$ & M7 & $-2.22 /-2.30$ & & $-2.54 /-2.58$ & & III \\
\hline J04153916+2818586 & $\ldots$ & M3.75 & $-1.56 /-1.65$ & $-0.95 /-1.00$ & $-1.49 /-1.53$ & $-0.77 /-0.79$ & II \\
\hline J04154278+2909597 & IRAS $04125+2902$ & M1.25 & $-2.56 /-2.64$ & $-1.00 /-1.04$ & $-2.82 /-2.85$ & $-0.71 /-0.73$ & II \\
\hline J04155799+2746175 & ... & M5.5 & $-1.24 /-1.24$ & $-1.05 /-1.05$ & $-1.29 /-1.29$ & $-1.03 /-1.03$ & II \\
\hline $\mathrm{J} 04161210+2756385$ & $\ldots$ & M4.75 & $-1.32 /-1.40$ & $-0.98 /-1.03$ & $-1.63 /-1.67$ & $-1.03 /-1.04$ & II \\
\hline J04161885+2752155 & $\ldots$ & M6.25 & $-2.31 /-2.35$ & $\ldots$ & $-2.57 /-2.59$ & $\ldots$ & III \\
\hline J04162725+2053091 & $\ldots$ & M5 & $-2.42 /-2.42$ & $\ldots$ & $-2.65 /-2.65$ & $\ldots$ & III \\
\hline $\mathrm{J} 04162810+2807358$ & $\mathrm{LkCa} 4$ & $\mathrm{~K} 7$ & $-2.57 /-2.59$ & $-2.74 /-2.75$ & $-2.60 /-2.61$ & $-2.79 /-2.80$ & III \\
\hline J04163048+3037053 & $\ldots$ & M4.5 & $-2.51 /-2.54$ & & $-2.78 /-2.80$ & & III \\
\hline J04163911+2858491 & $\ldots$ & M5.5 & $-1.45 /-1.57$ & $-1.33 /-1.39$ & $-1.65 /-1.70$ & $-1.38 /-1.40$ & II \\
\hline J04173372+2820468 & CY Tau & M1.5 & $-1.39 /-1.46$ & $-1.27 /-1.31$ & $-1.44 /-1.47$ & $-1.26 /-1.28$ & II \\
\hline J04173893+2833005 & $\mathrm{LkCa} 5$ & M2 & $-2.58 /-2.59$ & $-2.71 /-2.71$ & $-2.71 /-2.71$ & $-2.79 /-2.79$ & III \\
\hline J04174955+2813318 & KPNO 10 & M5 & $-1.38 /-1.44$ & $-1.01 /-1.05$ & $-0.58 /-0.61$ & $-0.58 /-0.60$ & II \\
\hline J04174965+2829362 & V410 X-ray 1 & M4 & $-0.88 /-1.14$ & $-0.88 /-1.02$ & $-0.67 /-0.79$ & $-0.79 /-0.84$ & II \\
\hline J04180796+2826036 & V410 X-ray 3 & M6 & $-2.34 /-2.38$ & $-2.39 /-2.41$ & $-2.66 /-2.68$ & $-2.54 /-2.55$ & III \\
\hline J04181078+2519574 & V409 Tau & M1.5 & $-0.83 /-1.02$ & $-1.13 /-1.23$ & $-0.83 /-0.92$ & $-1.21 /-1.25$ & II \\
\hline $\mathrm{J} 04181710+2828419$ & V410 Anon 13 & M5.75 & $-1.24 /-1.51$ & $-0.99 /-1.13$ & $-1.22 /-1.34$ & $-0.91 /-0.96$ & II \\
\hline J04182147+1658470 & HBC 372 & K5 & $-2.70 /-2.70$ & $\ldots$ & $-2.79 /-2.79$ & $\ldots$ & III \\
\hline J04182909+2826191 & V410 Anon 25 & M1 & $-1.68 /-2.69$ & $-2.19 /-2.73$ & $-2.27 /-2.74$ & $-2.57 /-2.76$ & III \\
\hline $\mathrm{J} 04183030+2743208$ & KPNO 11 & M5.5 & $-2.42 /-2.45$ & & $-2.65 /-2.66$ & & III \\
\hline $\mathrm{J} 04183110+2827162$ & V410 Tau $\mathrm{A}+\mathrm{B}+\mathrm{C}$ & K7 & $-2.50 /-2.50$ & $-2.69 /-2.69$ & $-2.69 /-2.69$ & $-2.82 /-2.82$ & III \\
\hline J04183112+2816290 & DD Tau A+B & M3.5 & $-0.30 /-0.54$ & $-0.50 /-0.62$ & $-0.45 /-0.56$ & $-0.61 /-0.65$ & II \\
\hline J04183158+2816585 & CZ Tau A+B & M3 & $0.37 / 0.32$ & $-0.02 /-0.05$ & $1.18 / 1.16$ & $0.22 / 0.21$ & II \\
\hline J04183203+2831153 & IRAS $04154+2823$ & M2.5 & $0.65 /-0.20$ & $0.34 /-0.12$ & $-0.23 /-0.62$ & $-0.11 /-0.27$ & I \\
\hline J04183444+2830302 & V410 X-ray 2 & M0 & $-1.62 /-2.56$ & $-0.64 /-1.15$ & $-2.07 /-2.50$ & $-0.58 /-0.77$ & II \\
\hline J04184023+2824245 & V410 X-ray 4 & M4 & $-1.88 /-2.68$ & $-2.25 /-2.69$ & $-2.36 /-2.74$ & $-2.56 /-2.71$ & III \\
\hline J04184061+2819155 & V892 Tau & B9 & & & & & II \\
\hline J04184133+2827250 & LR1 & K4.5 & $-0.56 /-1.65$ & $-0.40 /-0.98$ & $-1.18 /-1.68$ & $-0.62 /-0.83$ & II \\
\hline
\end{tabular}


Disk Population of Taurus

TABLE $7-$ Continued

\begin{tabular}{|c|c|c|c|c|c|c|c|}
\hline $2 \mathrm{MASS}^{\mathrm{a}}$ & Name & $\begin{array}{l}\text { Spectral } \\
\text { Type }\end{array}$ & $\alpha(2-8 \mu \mathrm{m})$ & $\alpha(2-24 \mu \mathrm{m})$ & $\alpha(3.6-8 \mu \mathrm{m})$ & $\alpha(3.6-24 \mu \mathrm{m})$ & $\begin{array}{l}\text { SED } \\
\text { Class }\end{array}$ \\
\hline J04184250+2818498 & V410 X-ray 7 & M0.75 & $-1.93 /-2.38$ & & $-1.99 /-2.20$ & & II \\
\hline J04184703+2820073 & Hubble 4 & K7 & $-2.53 /-2.64$ & $-2.65 /-2.71$ & $-2.61 /-2.67$ & $-2.71 /-2.74$ & III \\
\hline J04185115+2814332 & KPNO 2 & M7.5 & $-2.18 /-2.23$ & $\ldots$ & $-2.51 /-2.53$ & $\ldots$ & III \\
\hline J04185147+2820264 & $\mathrm{CoKu} \mathrm{Tau/1}$ & M0 & $0.94 / 0.67$ & $1.03 / 0.89$ & $2.17 / 2.05$ & $1.58 / 1.52$ & I \\
\hline J04185170+1723165 & HBC 376 & K7 & $-2.69 /-2.69$ & $-2.76 /-2.76$ & $-2.73 /-2.73$ & $-2.79 /-2.79$ & III \\
\hline J04185813+2812234 & IRAS $04158+2805$ & M5.25 & $0.30 /-0.03$ & $0.38 / 0.21$ & $-0.13 /-0.28$ & $0.22 / 0.16$ & I \\
\hline J04190110+2819420 & V410 X-ray 6 & M5.5 & $-2.16 /-2.32$ & $-0.76 /-0.85$ & $-2.23 /-2.30$ & $-0.44 /-0.47$ & II \\
\hline J04190126+2802487 & KPNO 12 & M9 & $-1.12 /-1.15$ & & $-1.33 /-1.34$ & & II \\
\hline J04190197+2822332 & V410 X-ray 5a & M5.5 & $-2.28 /-2.53$ & $-2.40 /-2.54$ & $-2.62 /-2.74$ & $-2.57 /-2.62$ & III \\
\hline J04191281+2829330 & FQ Tau A+B & M3 & $-1.42 /-1.47$ & $-1.16 /-1.19$ & $-1.35 /-1.38$ & $-1.07 /-1.08$ & II \\
\hline J04191583+2906269 & $\mathrm{BP} \mathrm{Tau}$ & K7 & $-1.30 /-1.36$ & $-0.86 /-0.90$ & $-0.97 /-1.00$ & $-0.62 /-0.63$ & II \\
\hline J04192625+2826142 & V819 Tau & K7 & $-2.53 /-2.59$ & $-2.08 /-2.11$ & $-2.69 /-2.72$ & $-2.03 /-2.04$ & II \\
\hline J04193545+2827218 & FR Tau & M5.25 & $-0.84 /-0.84$ & $-0.89 /-0.89$ & $-0.36 /-0.36$ & $-0.70 /-0.70$ & II \\
\hline J04194127+2749484 & $\mathrm{LkCa} 7 \mathrm{~A}+\mathrm{B}$ & M0 & $-2.57 /-2.59$ & $-2.69 /-2.70$ & $-2.73 /-2.74$ & $-2.79 /-2.79$ & III \\
\hline J04194148+2716070 & IRAS $04166+2708$ & $\mathrm{~K} 6-\mathrm{M} 3.5^{\mathrm{c}}$ & -0.39 & 0.75 & -0.64 & 0.93 & I \\
\hline & IRAS $04166+2706$ & $\mathrm{~K} 6-\mathrm{M} 3.5^{\mathrm{c}}$ & & & & & I \\
\hline J04194657+2712552 & [GKH94] 41 & M7.5 & $-0.07 /-1.20$ & $0.14 /-0.47$ & $-0.52 /-1.05$ & $0.00 /-0.22$ & $\mathrm{I} ?$ \\
\hline J04195844+2709570 & İRAS $04169+2702$ & $\mathrm{~K} 6-\mathrm{M} 3.5^{\mathrm{c}}$ & 1.72 & & 0.71 & & I \\
\hline J04201611+2821325 & $\ldots$ & M6.5 & $-1.36 /-1.36$ & $-1.19 /-1.19$ & $-1.40 /-1.40$ & $-1.17 /-1.17$ & II \\
\hline J04202144+2813491 & $\ldots$ & M1 & $-1.00 /-1.08$ & $-0.78 /-0.83$ & $-1.52 /-1.56$ & $-0.95 /-0.97$ & II \\
\hline J04202555+2700355 & & M5.25 & $-1.52 /-1.60$ & $-0.79 /-0.83$ & $-1.46 /-1.50$ & $-0.58 /-0.60$ & II \\
\hline J04202583+2819237 & IRAS $04173+2812$ & mid-M & $0.51 / 0.51$ & $0.18 / 0.18$ & $-0.48 /-0.48$ & $-0.32 /-0.32$ & II \\
\hline J04202606+2804089 & & M3.5 & $-0.87 /-0.87$ & $-0.39 /-0.39$ & $-0.23 /-0.23$ & $0.00 / 0.00$ & II \\
\hline J04203918+2717317 & XEST 16-045 & M4.5 & $-2.62 /-2.62$ & $-2.66 /-2.66$ & $-2.77 /-2.77$ & $-2.74 /-2.74$ & III \\
\hline J04205273+1746415 & $\mathrm{J} 2-157$ & M5.5 & $-2.44 /-2.44$ & & $-2.68 /-2.68$ & & III \\
\hline J04210795+2702204 & $\cdots$ & M5.25 & $0.92 / 0.44$ & $0.57 / 0.32$ & $0.08 /-0.14$ & $0.13 / 0.04$ & II \\
\hline J04210934+2750368 & $\ldots$ & M5.25 & $-1.96 /-1.96$ & $-1.65 /-1.65$ & $-1.94 /-1.94$ & $-1.55 /-1.55$ & II \\
\hline J04211038+2701372 & IRAS $04181+2654 \mathrm{~B}$ & K7 & 0.26 & 0.38 & -0.35 & 0.15 & I \\
\hline J04211146+2701094 & IRAS $04181+2654 \mathrm{~A}$ & M3 & 0.63 & 0.50 & 0.55 & 0.43 & I \\
\hline J04213459+2701388 & & M5.5 & $-1.74 /-1.81$ & $-1.61 /-1.65$ & $-1.77 /-1.80$ & $-1.59 /-1.60$ & II \\
\hline J04214013+2814224 & XEST 21-026 & M5.75 & $-2.43 /-2.43$ & $\ldots$ & $-2.73 /-2.73$ & $\ldots$ & III \\
\hline J04214323+1934133 & IRAS $04187+1927$ & M0 & $-0.16 /-0.45$ & $\ldots$ & $0.15 / 0.02$ & $\ldots$ & II \\
\hline J04214631+2659296 & $\ldots$ & M5.75 & $-1.62 /-1.71$ & $-1.01 /-1.06$ & $-1.68 /-1.71$ & $-0.88 /-0.90$ & II \\
\hline J04215450+2652315 & $\ldots$ & M8.5 & $-2.08 /-2.12$ & & $-2.55 /-2.57$ & & III \\
\hline J04215563+2755060 & DE Tau & M1 & $-1.27 /-1.33$ & $-0.86 /-0.89$ & $-1.32 /-1.34$ & $-0.78 /-0.79$ & II \\
\hline & IRAM 04191+1522 & & & & -0.16 & 1.17 & 0 \\
\hline J04215740+2826355 & RY Tau & K1 & $\ldots$ & $\cdots$ & & & II \\
\hline J04215884+2818066 & HD 283572 & G5 & $-2.71 /-2.74$ & $-2.83 /-2.85$ & $-2.77 /-2.79$ & $-2.89 /-2.89$ & III \\
\hline J04215943+1932063 & $\mathrm{T}$ Tau N+S & K0 & & $\ldots$ & & $\ldots$ & II \\
\hline J04220007+1530248 & IRAS $04191+1523$ B & $\mathrm{M} 6-\mathrm{M} 8^{\mathrm{c}}$ & 0.33 & $\ldots$ & -0.08 & $\ldots$ & I \\
\hline $\mathrm{J} 04220043+1530212$ & IRAS $04191+1523 \mathrm{~A}$ & $\mathrm{~K} 6-\mathrm{M} 3.5^{\mathrm{c}}$ & 1.47 & $1.09^{\mathrm{b}}$ & 0.82 & $0.77^{\mathrm{b}}$ & I \\
\hline J04220069+2657324 & Haro $6-5 \mathrm{~B}$ & K5 & 1.42 & 1.07 & 1.38 & 0.97 & I \\
\hline J04220217+2657304 & FS Tau A+B & M0 & $-0.53 /-0.84$ & $-0.19 /-0.36$ & $-0.79 /-0.93$ & $-0.21 /-0.27$ & II \\
\hline J04220313+2825389 & $\mathrm{LkCa} 21$ & M3 & $-2.54 /-2.56$ & $-2.66 /-2.68$ & $-2.70 /-2.71$ & $-2.76 /-2.77$ & III \\
\hline J04221332+1934392 & & M8 & $-2.12 /-2.12$ & & $-2.35 /-2.35$ & & III \\
\hline J04221568+2657060 & XEST 11-078 & M1 & $-1.14 /-1.18$ & $0.31 / 0.28$ & $-1.33 /-1.35$ & $0.59 / 0.59$ & $\mathrm{I} ?$ \\
\hline J04221644+2549118 & $\ldots$ & M7.75 & $-2.29 /-2.31$ & r & $-2.62 /-2.63$ & & III \\
\hline J04221675+2654570 & $\ldots$ & M1.5 & $-0.76 /-0.92$ & $-0.68 /-0.76$ & $-1.08 /-1.16$ & $-0.79 /-0.82$ & II \\
\hline J04222404+2646258 & XEST 11-087 & M4.75 & $-2.48 /-2.52$ & $-2.51 /-2.53$ & $-2.72 /-2.74$ & $-2.62 /-2.62$ & III \\
\hline J04224786+2645530 & IRAS $04196+2638$ & M1 & $-0.61 /-0.80$ & $-0.54 /-0.64$ & $-0.73 /-0.82$ & $-0.57 /-0.61$ & II \\
\hline J04230607+2801194 & $\ldots$ & M6 & $-1.46 /-1.48$ & $-1.03 /-1.04$ & $-1.42 /-1.43$ & $-0.90 /-0.91$ & II \\
\hline J04230776+2805573 & IRAS $04200+2759$ & $\mathrm{M} 3.5-\mathrm{M} 6^{\mathrm{c}}$ & $0.06 /-0.28$ & $-0.12 /-0.30$ & $-0.57 /-0.72$ & $-0.42 /-0.49$ & II \\
\hline J04231822+2641156 & & M3.5 & $-1.31 /-1.71$ & $-0.71 /-0.93$ & $-1.39 /-1.57$ & $-0.59 /-0.67$ & II \\
\hline J04233539+2503026 & FU Tau A & M7.25 & $-0.83 /-0.91$ & $-1.06 /-1.11$ & $-0.83 /-0.87$ & $-1.13 /-1.14$ & II \\
\hline J04233573+2502596 & FU Tau B & M9.25 & $-0.99 /-0.99$ & $\ldots$ & $-0.87 /-0.87$ & $\ldots$ & II \\
\hline J04233919+2456141 & FT Tau & $\mathrm{K} 6-\mathrm{M} 3.5^{\mathrm{c}}$ & $-1.03 /-1.16$ & $-0.78 /-0.85$ & $-0.96 /-1.02$ & $-0.69 /-0.71$ & II \\
\hline J04242090+2630511 & & M6.5 & $-1.31 /-1.31$ & $-0.97 /-0.97$ & $-1.20 /-1.20$ & $-0.84 /-0.84$ & II \\
\hline J04242646+2649503 & & M5.75 & $-1.41 /-1.43$ & $-0.96 /-0.97$ & $-1.39 /-1.40$ & $-0.84 /-0.84$ & II \\
\hline J04244457+2610141 & IRAS $04216+2603$ & M0.5 & $-0.70 /-0.89$ & $-0.74 /-0.85$ & $-0.75 /-0.84$ & $-0.78 /-0.81$ & II \\
\hline J04244506+2701447 & $\mathrm{J} 1-4423$ & M5 & $-2.50 /-2.52$ & $\ldots$ & $-2.71 /-2.71$ & $\ldots$ & III \\
\hline J04245708+2711565 & IP Tau & M0 & $-1.52 /-1.61$ & $-1.02 /-1.07$ & $-1.54 /-1.58$ & $-0.90 /-0.91$ & II \\
\hline $\mathrm{J} 04251767+2617504$ & J1-4872 B & M1 & $-2.13 /-2.13$ & & $-2.66 /-2.66$ & & III \\
\hline $\mathrm{J} 04251767+2617504$ & $\mathrm{~J} 1-4872 \mathrm{~A}$ & K7 & $-2.25 /-2.25$ & $-2.54 /-2.54^{\mathrm{b}}$ & $-2.71 /-2.71$ & $-2.83 /-2.83^{b}$ & III \\
\hline J04262939+2624137 & KPNO 3 & M6 & $-1.06 /-1.13$ & $-0.86 /-0.90$ & $-0.89 /-0.92$ & $-0.74 /-0.75$ & II \\
\hline J04263055+2443558 & $\ldots$ & M8.75 & $-1.11 /-1.11$ & $-1.15 /-1.15$ & $-1.11 /-1.11$ & $-1.16 /-1.16$ & II \\
\hline J04265352+2606543 & FV Tau A+B & K5 & $-0.53 /-0.87$ & $-0.56 /-0.75$ & $-0.61 /-0.77$ & $-0.61 /-0.68$ & II \\
\hline J04265440+2606510 & FV Tau/c A+B & M2.5 & $-0.94 /-1.21$ & & $-0.81 /-0.93$ & & II \\
\hline J04265629+2443353 & IRAS $04239+2436$ & $\mathrm{~K} 6-\mathrm{M} 3.5^{\mathrm{c}}$ & 1.18 & & 0.76 & & I \\
\hline J04265732+2606284 & KPNO 13 & M5 & $-1.11 /-1.22$ & $-1.22 /-1.28$ & $-1.18 /-1.23$ & $-1.28 /-1.30$ & II \\
\hline J04270266+2605304 & DG Tau B & $<\mathrm{K} 6^{\mathrm{c}}$ & $\ldots$ & $\ldots$ & 1.87 & $\ldots$ & I \\
\hline J04270280+2542223 & DF Tau A+B & M2 & $-1.20 /-1.28$ & $-1.14 /-1.18$ & $-1.11 /-1.14$ & $-1.08 /-1.10$ & II \\
\hline J04270469+2606163 & DG Tau & K6 & $-0.33 /-0.49$ & $\ldots$ & & $\ldots$ & II \\
\hline J04270739+2215037 & & M6.75 & $-2.39 /-2.40$ & $\cdots$ & $-2.66 /-2.67$ & $\cdots$ & III \\
\hline J04272799+2612052 & KPNO 4 & M9.5 & $-1.96 /-1.96$ & $\ldots$ & $-2.35 /-2.35$ & $\ldots$ & III \\
\hline
\end{tabular}


TABLE $7-$ Continued

\begin{tabular}{|c|c|c|c|c|c|c|c|}
\hline $2 \mathrm{MASS}^{\mathrm{a}}$ & Name & $\begin{array}{c}\text { Spectral } \\
\text { Type }\end{array}$ & $\alpha(2-8 \mu \mathrm{m})$ & $\alpha(2-24 \mu \mathrm{m})$ & $\alpha(3.6-8 \mu \mathrm{m})$ & $\alpha(3.6-24 \mu \mathrm{m})$ & $\begin{array}{l}\text { SED } \\
\text { Class }\end{array}$ \\
\hline J04274538+2357243 & & M8.25 & $-2.30 /-2.30$ & & $-2.63 /-2.63$ & & III \\
\hline J04275730+2619183 & IRAS $04248+2612$ & M4.5 & $0.10 /-0.21$ & $0.51 / 0.34$ & $0.24 / 0.09$ & $0.67 / 0.61$ & I \\
\hline & L1521F-IRS & $\mathrm{M} 6-\mathrm{M} 8^{\mathrm{c}}$ & & & & & \\
\hline J04284263+2714039 & & M5.25 & $-1.63 /-1.65$ & $-1.26 /-1.27$ & $-1.86 /-1.87$ & $-1.26 /-1.27$ & II \\
\hline J04290068+2755033 & $\cdots$ & M8.25 & $-1.43 /-1.43$ & $-1.04 /-1.04$ & $-1.36 /-1.36$ & $-0.91 /-0.91$ & II \\
\hline J04290498+2649073 & IRAS $04260+2642$ & K5.5 & -0.04 & 0.36 & -0.58 & 0.24 & II \\
\hline J04292071+2633406 & $\mathrm{J} 1-507$ & M4 & $-2.54 /-2.59$ & $-2.64 /-2.67$ & $-2.75 /-2.78$ & $-2.76 /-2.77$ & III \\
\hline J04292165+2701259 & IRAS $04263+2654$ & M5.25 & $-1.29 /-1.45$ & $-0.83 /-0.92$ & $-1.20 /-1.28$ & $-0.68 /-0.71$ & II \\
\hline J04292373+2433002 & GV Tau A+B & K5 & $\ldots$ & & & & I \\
\hline J04292971+2616532 & FW Tau A+B+C & M5.5 & $-2.42 /-2.46$ & $-2.20 /-2.22$ & $-2.64 /-2.66$ & $-2.24 /-2.25$ & III \\
\hline J04293008+2439550 & IRAS $04264+2433$ & M1 & 0.35 & 1.02 & 1.08 & 1.50 & I \\
\hline J04293209+2430597 & & $\mathrm{K} 6-\mathrm{M} 3.5^{\mathrm{c}}$ & 1.82 & 0.90 & 0.71 & 0.21 & $\mathrm{I} ?$ \\
\hline J04293606+2435556 & XEST 13-010 & M3 & $-1.60 /-1.72$ & $-1.13 /-1.20$ & $-1.71 /-1.77$ & $-1.06 /-1.08$ & II \\
\hline J04294155+2632582 & $\mathrm{DH}$ Tau A+B & M1 & $-1.79 /-1.89$ & $-1.00 /-1.05$ & $-1.97 /-2.02$ & $-0.87 /-0.89$ & II \\
\hline J04294247+2632493 & DI Tau A+B & M0 & $-2.59 /-2.60$ & $\ldots$ & $-2.76 /-2.76$ & $\ldots$ & III \\
\hline J04294568+2630468 & KPNO 5 & M7.5 & $-2.30 /-2.33$ & $\ldots$ & $-2.62 /-2.64$ & & III \\
\hline J04295156+2606448 & IQ Tau & M0.5 & $-1.17 /-1.29$ & $-0.97 /-1.04$ & $-1.32 /-1.38$ & $-0.98 /-1.01$ & II \\
\hline J04295422+1754041 & & M4 & & & & & III? \\
\hline J04295950+2433078 & $\ldots$ & M5 & $-1.35 /-1.44$ & $-0.98 /-1.03$ & $-1.43 /-1.47$ & $-0.92 /-0.94$ & II \\
\hline J04300357+1813494 & UX Tau B & M2 & $-2.60 /-2.64$ & & $-2.75 /-2.77$ & & III \\
\hline J04300399+1813493 & UX Tau A+C & K5 & $-1.63 /-1.69$ & $-0.78 /-0.81^{b}$ & $-2.01 /-2.04$ & $-0.66 /-0.68^{\mathrm{b}}$ & II \\
\hline J04300724+2608207 & KPNO 6 & M8.5 & $-1.36 /-1.39$ & $-1.14 /-1.16$ & $-1.26 /-1.27$ & $-1.05 /-1.05$ & II \\
\hline J04302365+2359129 & & M8.25 & $-2.31 /-2.31$ & & $-2.64 /-2.64$ & & III \\
\hline $\mathrm{J} 04302961+2426450$ & FX Tau A+B & M1 & $-1.35 /-1.45$ & $-0.98 /-1.03$ & $-1.38 /-1.43$ & $-0.90 /-0.92$ & II \\
\hline $\mathrm{J} 04304425+2601244$ & DK Tau A & $\mathrm{K} 7$ & $-0.77 /-0.90$ & $-0.81 /-0.88^{\mathrm{b}}$ & $-1.25 /-1.31$ & $-0.99 /-1.02^{\mathrm{b}}$ & II \\
\hline J04304425+2601244 & DK Tau B & $\mathrm{K} 6-\mathrm{M} 3.5^{\mathrm{c}}$ & -1.08 & $\ldots$ & -1.35 & $\ldots$ & II \\
\hline J04305028+2300088 & IRAS $04278+2253 \mathrm{~A}+\mathrm{B}$ & G8 & $\ldots$ & $\ldots$ & $\ldots$ & $\ldots$ & I \\
\hline J04305137+2442222 & ZZ Tau & M3 & $-1.74 /-1.81$ & $-1.36 /-1.39$ & $-1.58 /-1.61$ & $-1.19 /-1.21$ & II \\
\hline J04305171+2441475 & ZZ Tau IRS & M5 & $0.50 / 0.18$ & $0.31 / 0.14$ & $-0.11 /-0.26$ & $0.01 /-0.06$ & II \\
\hline J04305718+2556394 & KPNO 7 & M8.25 & $-1.34 /-1.36$ & $-1.02 /-1.03$ & $-1.31 /-1.32$ & $-0.92 /-0.93$ & II \\
\hline J04311444+2710179 & JH 56 & M0.5 & $-2.67 /-2.67$ & $-2.08 /-2.08$ & $-2.72 /-2.72$ & $-1.96 /-1.96$ & II \\
\hline J04311578+1820072 & MHO 9 & M4.25 & $-2.51 /-2.52$ & $-2.69 /-2.70$ & $-2.71 /-2.72$ & $-2.82 /-2.82$ & III \\
\hline J04311907+2335047 & & M7.75 & $-2.27 /-2.29$ & $\ldots$ & $-2.65 /-2.66$ & & III \\
\hline J04312382+2410529 & V927 Tau A+B & M4.75 & $-2.52 /-2.55$ & $-2.68 /-2.69$ & $-2.70 /-2.71$ & $-2.79 /-2.80$ & III \\
\hline $\mathrm{J} 04312405+1800215$ & MHO 4 & M7 & $-2.34 /-2.38$ & $-2.52 /-2.54$ & $-2.64 /-2.67$ & $-2.69 /-2.70$ & III \\
\hline J04312669+2703188 & & M7.5 & $-2.24 /-2.26$ & $\ldots$ & $-2.64 /-2.65$ & $\ldots$ & III \\
\hline J04313407+1808049 & L1551/IRS5 & $<\mathrm{K} 6^{\mathrm{c}}$ & 2.27 & $\ldots$ & 2.06 & $\ldots$ & $\mathrm{I}$ \\
\hline J04313613+1813432 & LkHa 358 & $\mathrm{~K} 8$ & $-0.45 /-0.92$ & $-0.13 /-0.39$ & $-0.52 /-0.74$ & $-0.08 /-0.17$ & I \\
\hline J04313747+1812244 & HH 30 & M0 & -1.27 & -0.28 & -1.55 & -0.15 & I \\
\hline J04313843+1813576 & HL Tau & $\mathrm{K} 7$ & $0.43 / 0.10$ & $\ldots$ & $\ldots$ & $\ldots$ & I \\
\hline J04314007+1813571 & XZ Tau A+B & M2 & $-0.11 /-0.28$ & $\ldots$ & $\ldots$ & & II \\
\hline J04314444+1808315 & L1551NE & $<\mathrm{K} 6^{\mathrm{c}}$ & 1.80 & 1.31 & 1.34 & 1.00 & $\mathrm{I}$ \\
\hline $\mathrm{J} 04315056+2424180$ & HK Tau A+B & M0.5 & $-1.33 /-1.54$ & $-0.46 /-0.57$ & $-1.50 /-1.60$ & $-0.31 /-0.35$ & II \\
\hline $\mathrm{J} 04315779+1821380$ & V710 Tau A & M0.5 & $-1.07 /-1.07$ & $-1.26 /-1.26^{\mathrm{b}}$ & $-1.01 /-1.01$ & $-1.13 /-1.13^{\mathrm{b}}$ & II \\
\hline J04315779+1821350 & V710 Tau B & M2 & -2.37 & & -2.36 & & II? \\
\hline J04315844+2543299 & $\mathrm{J} 1-665$ & M5.5 & $-2.56 /-2.64$ & $-2.77 /-2.81$ & $-2.78 /-2.81$ & $-2.91 /-2.92$ & III \\
\hline J04315968+1821305 & LkHa 267 & M1.5 & $-0.83 /-1.04$ & $-0.75 /-0.86$ & $-1.15 /-1.25$ & $-0.87 /-0.91$ & II \\
\hline J04320329+2528078 & $\ldots$ & M6.25 & $-2.34 /-2.34$ & $\ldots$ & $-2.64 /-2.64$ & $\ldots$ & III \\
\hline J04320926+1757227 & L1551-51 & K7 & $-2.65 /-2.66$ & $-2.81 /-2.81$ & $-2.70 /-2.70$ & $-2.86 /-2.86$ & III \\
\hline J04321456+1820147 & V827 Tau & K7 & $-2.64 /-2.67$ & $-2.75 /-2.76$ & $-2.74 /-2.75$ & $-2.81 /-2.82$ & III \\
\hline J04321540+2428597 & Haro 6-13 & M0 & $-0.35 /-0.76$ & & $-1.05 /-1.24$ & & II \\
\hline J04321583+1801387 & V826 Tau A+B & K7 & $-2.59 /-2.60$ & $-2.73 /-2.73$ & $-2.77 /-2.77$ & $-2.84 /-2.84$ & III \\
\hline J04321606+1812464 & MHO 5 & M6 & $-1.20 /-1.25$ & $-1.18 /-1.21$ & $-1.18 /-1.20$ & $-1.17 /-1.18$ & II \\
\hline J04321786+2422149 & & M5.75 & $-2.32 /-2.32$ & $-2.62 /-2.62$ & $-2.61 /-2.61$ & $-2.82 /-2.82$ & III \\
\hline J04321885+2422271 & V928 Tau A+B & M0.5 & $-2.46 /-2.62$ & $-2.68 /-2.77$ & $-2.63 /-2.70$ & $-2.80 /-2.83$ & III \\
\hline J04322210+1827426 & MHO 6 & M4.75 & $-1.65 /-1.69$ & $-1.23 /-1.25$ & $-1.71 /-1.73$ & $-1.14 /-1.15$ & II \\
\hline J04322329+2403013 & $\ldots$ & M7.75 & $-2.33 /-2.33$ & & $-2.58 /-2.58$ & & III \\
\hline $\mathrm{J} 04322415+2251083$ & $\ldots$ & M4.5 & $-1.31 /-1.39$ & $-1.18 /-1.22$ & $-1.09 /-1.12$ & $-1.05 /-1.06$ & II \\
\hline J04322627+1827521 & MHO 7 & M5.25 & $-2.48 /-2.51$ & $-2.75 /-2.77$ & $-2.70 /-2.71$ & $-2.91 /-2.92$ & III \\
\hline J04323028+1731303 & GG Tau $\mathrm{Ba}+\mathrm{Bb}$ & M5.5 & $-1.10 /-1.13$ & $\ldots$ & $-1.02 /-1.04$ & $\ldots$ & II \\
\hline J04323034+1731406 & GG Tau $\mathrm{Aa}+\mathrm{Ab}$ & K7 & $-1.11 /-1.20$ & $\ldots$ & $-1.01 /-1.06$ & $\ldots$ & II \\
\hline J04323058+2419572 & FY Tau & K5 & $-1.31 /-1.61$ & $-1.22 /-1.38$ & $-1.51 /-1.64$ & $-1.27 /-1.33$ & II \\
\hline J04323176+2420029 & FZ Tau & M0 & $-0.74 /-1.05$ & $-0.79 /-0.96$ & $-0.78 /-0.92$ & $-0.83 /-0.89$ & II \\
\hline J04323205+2257266 & IRAS $04295+2251$ & K7 & 0.62 & 0.58 & 0.82 & 0.65 & $\mathrm{I}$ \\
\hline $\mathrm{J} 04324282+2552314$ & UZ Tau $\mathrm{Ba}+\mathrm{Bb}$ & M2 & $-1.45 /-1.57$ & & $-1.40 /-1.46$ & & II \\
\hline $\mathrm{J} 04324303+2552311$ & UZ Tau A & M1 & $-0.53 /-0.80$ & $-0.73 /-0.87^{\mathrm{b}}$ & $-0.78 /-0.90$ & $-0.79 /-0.84^{\mathrm{b}}$ & II \\
\hline J04324373+1802563 & L1551-55 & $\mathrm{K} 7$ & $-2.64 /-2.67$ & $-2.80 /-2.81$ & $-2.74 /-2.76$ & $-2.88 /-2.89$ & III \\
\hline $\mathrm{J} 04324911+2253027$ & JH 112 & K6 & $-1.13 /-1.40$ & $-0.70 /-0.84$ & $-1.08 /-1.20$ & $-0.57 /-0.62$ & II \\
\hline J04324938+2253082 & $\ldots$ & M4.25 & $-1.24 /-1.37$ & $\ldots$ & $-1.18 /-1.24$ & $\ldots$ & II \\
\hline J04325026+2422115 & $\ldots$ & M7.5 & $-1.90 /-2.36$ & $-2.17 /-2.42$ & $-2.40 /-2.62$ & $-2.46 /-2.55$ & III \\
\hline J04325119+1730092 & LH $0429+17$ & M8.25 & $-2.21 /-2.21$ & & & & III \\
\hline J04330197+2421000 & MHO 8 & M6 & $-2.33 /-2.41$ & $-2.55 /-2.59$ & $-2.62 /-2.66$ & $-2.73 /-2.74$ & III \\
\hline J04330622+2409339 & GH Tau A+B & M2 & $-1.47 /-1.53$ & $-1.12 /-1.16$ & $-1.60 /-1.63$ & $-1.09 /-1.10$ & II \\
\hline J04330664+2409549 & V807 Tau A+B & K5 & $-1.71 /-1.76$ & $-1.36 /-1.39$ & $-1.74 /-1.76$ & $-1.28 /-1.29$ & II \\
\hline
\end{tabular}


Disk Population of Taurus

TABLE 7 - Continued

\begin{tabular}{|c|c|c|c|c|c|c|c|}
\hline $2 \mathrm{MASS}^{\mathrm{a}}$ & Name & $\begin{array}{l}\text { Spectral } \\
\text { Type }\end{array}$ & $\alpha(2-8 \mu \mathrm{m})$ & $\alpha(2-24 \mu \mathrm{m})$ & $\alpha(3.6-8 \mu \mathrm{m})$ & $\alpha(3.6-24 \mu \mathrm{m})$ & $\begin{array}{l}\text { SED } \\
\text { Class }\end{array}$ \\
\hline J04330781+2616066 & KPNO 14 & M6 & $-2.28 /-2.41$ & $-2.49 /-2.55$ & $-2.63 /-2.69$ & $-2.68 /-2.71$ & III \\
\hline J04330945+2246487 & & M6 & $-1.61 /-1.72$ & $-1.61 /-1.67$ & $-1.83 /-1.88$ & $-1.70 /-1.73$ & II \\
\hline J04331003+2433433 & V830 Tau & K7 & $-2.71 /-2.74$ & $-2.81 /-2.83$ & $-2.79 /-2.80$ & $-2.87 /-2.88$ & III \\
\hline J04331435+2614235 & IRAS $04301+2608$ & M0 & $-0.64 /-0.89$ & $0.67 / 0.54$ & $-0.02 /-0.14$ & $1.27 / 1.22$ & II \\
\hline $\mathrm{J} 04331650+2253204$ & IRAS $04302+2247$ & $\mathrm{~K} 6-\mathrm{M} 3.5^{\mathrm{c}}$ & -1.29 & 0.29 & -2.26 & 0.29 & I \\
\hline $\mathrm{J} 04331907+2246342$ & IRAS $04303+2240$ & M0.5 & $-0.04 /-0.54$ & $-0.44 /-0.72$ & $-0.18 /-0.42$ & $-0.61 /-0.71$ & II \\
\hline J04332621+2245293 & XEST 17-036 & M4 & $-2.37 /-2.53$ & $-2.26 /-2.35$ & $-2.62 /-2.69$ & $-2.34 /-2.37$ & III \\
\hline J04333278+1800436 & & M1 & $-1.16 /-1.16$ & $\ldots$ & … & $\ldots$ & II \\
\hline J04333297+1801004 & HD 28867 B & B9.5 & -2.35 & $\ldots$ & $\ldots$ & $\ldots$ & II \\
\hline J04333297+1801004 & HD $28867 \mathrm{~A}+\mathrm{C}$ & B9 & -2.77 & $\ldots$ & $\ldots$ & & III \\
\hline $\mathrm{J} 04333405+2421170$ & GI Tau & K7 & $-0.55 /-0.66$ & $-0.62 /-0.68$ & $-0.50 /-0.56$ & $-0.62 /-0.64$ & II \\
\hline J04333456+2421058 & GK Tau & K7 & $-0.89 /-0.99$ & $-0.64 /-0.70$ & $-1.00 /-1.04$ & $-0.62 /-0.64$ & II \\
\hline J04333678+2609492 & IS Tau $\mathrm{A}+\mathrm{B}$ & M0 & $-0.90 /-1.04$ & $-0.97 /-1.04$ & $-0.82 /-0.88$ & $-0.95 /-0.98$ & II \\
\hline J04333905+2227207 & & M1.75 & $-1.68 /-1.74$ & $-0.63 /-0.66$ & $-1.93 /-1.95$ & $-0.47 /-0.48$ & II \\
\hline $\mathrm{J} 04333906+2520382$ & DL Tau & K7 & $-0.64 /-0.77$ & $-0.64 /-0.70$ & $-0.64 /-0.70$ & $-0.64 /-0.66$ & II \\
\hline J04333935+1751523 & HN Tau A+B & K5 & $-0.14 /-0.41$ & $\ldots$ & $-0.29 /-0.41$ & $\ldots$ & II \\
\hline J04334171+1750402 & .. & M4 & $-1.90 /-1.91$ & $\ldots$ & $-1.81 /-1.81$ & $\ldots$ & II \\
\hline $\mathrm{J} 04334291+2526470$ & $\ldots$ & M8.75 & $-2.20 /-2.20$ & $\ldots$ & $-2.64 /-2.64$ & $\ldots$ & III \\
\hline J04334465+2615005 & $\ldots$ & M4.75 & $-0.94 /-1.07$ & $-0.87 /-0.94$ & $-1.04 /-1.10$ & $-0.89 /-0.92$ & II \\
\hline J04334871+1810099 & DM Tau & M1 & $-2.20 /-2.23$ & & $-2.11 /-2.12$ & & II \\
\hline $\mathrm{J} 04335200+2250301$ & CI Tau & K7 & $-0.93 /-1.03$ & $-0.70 /-0.75$ & $-0.87 /-0.92$ & $-0.61 /-0.63$ & II \\
\hline $\mathrm{J} 04335245+2612548$ & & M8.5 & $-0.98 /-1.12$ & $-0.67 /-0.74$ & $-0.94 /-1.01$ & $-0.57 /-0.60$ & II \\
\hline J04335252+2256269 & XEST 17-059 & M5.75 & $-2.42 /-2.42$ & $-2.61 /-2.61$ & $-2.63 /-2.63$ & $-2.75 /-2.75$ & III \\
\hline J04335470+2613275 & IT Tau B & $\mathrm{M} 3.5-\mathrm{M} 6^{\mathrm{c}}$ & -0.94 & $\ldots$ & -0.87 & $\ldots$ & II \\
\hline $\mathrm{J} 04335470+2613275$ & IT Tau A & $\mathrm{K} 2$ & $-1.31 /-1.63$ & $-1.21 /-1.38^{\mathrm{b}}$ & $-1.35 /-1.50$ & $-1.20 /-1.27^{\mathrm{b}}$ & II \\
\hline J04335546+1838390 & $\mathrm{J} 2-2041$ & M3.5 & $-2.56 /-2.58$ & & $-2.74 /-2.75$ & & III \\
\hline J04341099+2251445 & JH 108 & M1 & $-2.60 /-2.67$ & $-2.62 /-2.65$ & $-2.72 /-2.75$ & $-2.67 /-2.68$ & III \\
\hline J04341527+2250309 & CFHT 1 & M7 & $-2.17 /-2.41$ & & $-2.61 /-2.72$ & . & III \\
\hline J04341803+1830066 & HBC 407 & G8 & -2.70 & $\ldots$ & -2.79 & $\ldots$ & III \\
\hline J04344544+2308027 & $\ldots$ & M5.25 & $-2.35 /-2.42$ & $\ldots$ & $-2.67 /-2.70$ & $\ldots$ & III \\
\hline J04345542+2428531 & AA Tau & K7 & $-1.03 /-1.10$ & $-0.85 /-0.89$ & $-0.94 /-0.97$ & $-0.77 /-0.78$ & II \\
\hline J04345693+2258358 & XEST 08-003 & M1.5 & $-2.56 /-2.64$ & $-2.52 /-2.57$ & $-2.72 /-2.76$ & $-2.58 /-2.60$ & III \\
\hline J04350850+2311398 & & M6 & $-2.35 /-2.35$ & $\ldots$ & $-2.61 /-2.61$ & & III \\
\hline J04352020+2232146 & HO Tau & M0.5 & $-1.39 /-1.45$ & $-1.02 /-1.05$ & $-1.52 /-1.55$ & $-0.98 /-0.99$ & II \\
\hline J04352089+2254242 & FF Tau A+B & K7 & $-2.61 /-2.69$ & $-2.74 /-2.79$ & $-2.81 /-2.85$ & $-2.86 /-2.88$ & III \\
\hline $\mathrm{J} 04352450+1751429$ & HBC $412 \mathrm{~A}+\mathrm{B}$ & M2 & $-2.59 /-2.61$ & $-2.76 /-2.77$ & $-2.73 /-2.73$ & $-2.86 /-2.86$ & III \\
\hline $\mathrm{J} 04352737+2414589$ & DN Tau & MO & $-1.34 /-1.39$ & $-0.94 /-0.97$ & $-1.18 /-1.20$ & $-0.77 /-0.78$ & II \\
\hline$\ldots$ & IRAS $04325+2402 \mathrm{C}$ & $\mathrm{M} 6-\mathrm{M} 8^{\mathrm{c}}$ & -0.86 & $\ldots$ & -1.07 & $\ldots$ & II \\
\hline J04353539+2408194 & IRAS $04325+2402 \mathrm{~A}+\mathrm{B}$ & $\mathrm{K} 6-\mathrm{M} 3.5^{\mathrm{c}}$ & -0.56 & $1.08^{\mathrm{b}}$ & -1.25 & $1.19^{\mathrm{b}}$ & I \\
\hline J04354093+2411087 & CoKu Tau $3 \mathrm{~A}+\mathrm{B}$ & M1 & $-0.75 /-1.11$ & $-0.89 /-1.08$ & $-0.84 /-1.01$ & $-0.96 /-1.03$ & II \\
\hline J04354183+2234115 & KPNO 8 & M5.75 & $-2.38 /-2.40$ & $\ldots$ & $-2.65 /-2.66$ & $\ldots$ & III \\
\hline J04354203+2252226 & XEST 08-033 & M4.75 & $-2.44 /-2.51$ & $-2.46 /-2.50$ & $-2.68 /-2.71$ & $-2.57 /-2.59$ & III \\
\hline $\mathrm{J} 04354526+2737130$ & & M9.25 & $-2.31 /-2.31$ & & $-2.76 /-2.76$ & & III \\
\hline J04354733+2250216 & HQ Tau & $\mathrm{K} 2$ & $-0.90 /-1.08$ & $-0.69 /-0.79$ & $-0.33 /-0.42$ & $-0.40 /-0.43$ & II \\
\hline J04355109+2252401 & KPNO 15 & M2.75 & $-2.54 /-2.62$ & $\ldots$ & $-2.74 /-2.78$ & $\ldots$ & III \\
\hline $\mathrm{J} 04355143+2249119$ & KPNO 9 & M8.5 & $-2.26 /-2.33$ & $\ldots$ & $-2.65 /-2.69$ & $\ldots$ & III \\
\hline $\mathrm{J} 04355209+2255039$ & XEST 08-047 & M4.5 & $-2.47 /-2.55$ & $\ldots$ & $-2.66 /-2.70$ & 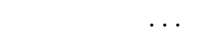 & III \\
\hline J04355277+2254231 & HP Tau & K3 & $-0.70 /-0.95$ & $-0.40 /-0.54$ & $-0.76 /-0.88$ & $-0.35 /-0.40$ & II \\
\hline J04355286+2250585 & XEST 08-049 & M4.25 & $-2.45 /-2.50$ & $-2.56 /-2.59$ & $-2.69 /-2.71$ & $-2.68 /-2.69$ & III \\
\hline J04355349+2254089 & HP Tau/G3 & K7 & $-2.57 /-2.67$ & $\ldots$ & $-2.74 /-2.78$ & $\ldots$ & III \\
\hline $\mathrm{J} 04355415+2254134$ & HP Tau/G2 & G0 & $-2.64 /-2.76$ & & $-2.69 /-2.75$ & & III \\
\hline J04355684+2254360 & Haro 6-28 A+B & M3 & $-1.08 /-1.25$ & $-0.90 /-1.00$ & $-1.10 /-1.19$ & $-0.87 /-0.91$ & II \\
\hline J04355892+2238353 & XEST 09-042 & M0 & $-2.57 /-2.59$ & & $-2.81 /-2.81$ & & III \\
\hline J04361030+2159364 & $\ldots$ & M8.5 & $-1.43 /-1.43$ & $-0.94 /-0.94$ & $-1.44 /-1.44$ & $-0.82 /-0.82$ & II \\
\hline J04361038+2259560 & CFHT 2 & M7.5 & $-2.19 /-2.27$ & & $-2.55 /-2.59$ & & III \\
\hline J04361909+2542589 & LkCa 14 & M0 & $-2.70 /-2.70$ & $-2.78 /-2.78$ & $-2.82 /-2.82$ & $-2.86 /-2.86$ & III \\
\hline J04362151+2351165 & & M5.25 & $-1.58 /-1.63$ & $-1.18 /-1.21$ & $-1.33 /-1.36$ & $-0.98 /-0.99$ & II \\
\hline J04363893+2258119 & CFHT 3 & M7.75 & $-2.21 /-2.28$ & $\ldots$ & $-2.60 /-2.64$ & $\ldots$ & III \\
\hline J04373705+2331080 & & Lo & $-1.26 /-1.26$ & & $-1.87 /-1.87$ & & III \\
\hline J04375670+2546229 & ITG 1 & $\mathrm{M} 3.5-\mathrm{M} 6^{\mathrm{c}}$ & $-0.77 /-0.77$ & $-0.73 /-0.73$ & $-0.47 /-0.47$ & $-0.60 /-0.60$ & II \\
\hline J04380083+2558572 & ITG 2 & M7.25 & $-2.25 /-2.39$ & $-2.56 /-2.63$ & $-2.59 /-2.65$ & $-2.78 /-2.81$ & III \\
\hline J04381486+2611399 & $\ldots$ & M7.25 & $0.14 / 0.14$ & $0.19 / 0.19$ & $-0.68 /-0.68$ & $-0.14 /-0.14$ & II \\
\hline $\mathrm{J} 04381630+2326402$ & $\ldots$ & M4.75 & & & & & III \\
\hline J04382134+2609137 & GM Tau & M6.5 & $-0.83 /-1.08$ & $-0.83 /-0.97$ & $-1.19 /-1.30$ & $-0.98 /-1.03$ & II \\
\hline J04382858+2610494 & DO Tau & M0 & $-0.65 /-0.86$ & $-0.39 /-0.50$ & $-0.74 /-0.83$ & $-0.36 /-0.40$ & II \\
\hline J04383528+2610386 & HV Tau A+B & M1 & $-2.45 /-2.56$ & $\ldots$ & $-2.56 /-2.61$ & $\ldots$ & III \\
\hline ... & HV Tau C & $\mathrm{K} 6$ & $\ldots$ & & -0.53 & 0.87 & I? \\
\hline J04385859+2336351 & & M4.25 & $\ldots$ & $-1.09 /-1.09$ & $\ldots$ & $-0.95 /-0.95$ & II \\
\hline J04385871+2323595 & $\ldots$ & M6.5 & $\ldots$ & $\ldots$ & $\ldots$ & $\ldots$ & III \\
\hline J04390163+2336029 & $\ldots$ & M6 & $\ldots$ & $-1.36 /-1.40$ & $\ldots$ & $-1.25 /-1.26$ & II \\
\hline J04390396+2544264 & $\ldots$ & M7.25 & $-1.20 /-1.27$ & $-0.98 /-1.01$ & $-0.98 /-1.01$ & $-0.83 /-0.84$ & II \\
\hline $\mathrm{J} 04390525+2337450$ & $\ldots$ & $\mathrm{M} 3.5-\mathrm{M} 6^{\mathrm{c}}$ & $-0.97 /-0.97$ & $0.24 / 0.24$ & $-1.10 /-1.10$ & $0.50 / 0.50$ & $\mathrm{I}$ ? \\
\hline J04390637+2334179 & $\ldots$ & M7.5 & $\ldots$ & $\ldots$ & $\ldots$ & $\ldots$ & III \\
\hline J04391389+2553208 & IRAS $04361+2547$ & $<\mathrm{K} 6^{\mathrm{c}}$ & 0.83 & $\ldots$ & 0.24 & $\ldots$ & $\mathrm{I}$ \\
\hline
\end{tabular}


TABLE $7-$ Continued

\begin{tabular}{|c|c|c|c|c|c|c|c|}
\hline $2 \mathrm{MASS}^{\mathrm{a}}$ & Name & $\begin{array}{c}\text { Spectral } \\
\text { Type }\end{array}$ & $\alpha(2-8 \mu \mathrm{m})$ & $\alpha(2-24 \mu \mathrm{m})$ & $\alpha(3.6-8 \mu \mathrm{m})$ & $\alpha(3.6-24 \mu \mathrm{m})$ & $\begin{array}{l}\text { SED } \\
\text { Class }\end{array}$ \\
\hline J04391741+2247533 & VY Tau A+B & M0 & $-1.69 /-1.71$ & $-1.20 /-1.22$ & $-1.48 /-1.49$ & $-0.99 /-1.00$ & II \\
\hline J04391779+2221034 & LkCa 15 & K5 & $-1.63 /-1.68$ & $-0.92 /-0.95$ & $-1.59 /-1.62$ & $-0.73 /-0.74$ & II \\
\hline J04392090+2545021 & GN Tau A+B & M2.5 & $-0.95 /-1.20$ & $-0.86 /-1.00$ & $-1.07 /-1.18$ & $-0.89 /-0.94$ & II \\
\hline J04393364+2359212 & & M5 & $-1.09 /-1.14$ & $-0.93 /-0.96$ & $-0.89 /-0.92$ & $-0.81 /-0.82$ & II \\
\hline J04393519+2541447 & IRAS $04365+2535$ & $<\mathrm{K} 6^{\mathrm{c}}$ & 1.95 & & 0.78 & & I \\
\hline J04394488+2601527 & ITG 15 & M5 & $-1.46 /-1.65$ & $-0.95 /-1.05$ & $-1.31 /-1.40$ & $-0.76 /-0.79$ & II \\
\hline \multirow[t]{2}{*}{ J04394748+2601407 } & CFHT 4 & M7 & $-0.99 /-1.21$ & $-0.81 /-0.93$ & $-0.93 /-1.03$ & $-0.74 /-0.78$ & II \\
\hline & IRAS $04368+2557$ & & & & 1.66 & 2.23 & 0 \\
\hline J04395574+2545020 & IC2087IR & $<\mathrm{K} 6^{\mathrm{c}}$ & -0.26 & & & & I \\
\hline $\mathrm{J} 04400067+2358211$ & $\ldots$ & M6 & $-1.50 /-1.50$ & $-0.91 /-0.91$ & $-1.46 /-1.46$ & $-0.74 /-0.74$ & II \\
\hline J04400174+2556292 & $\ldots$ & M5.5 & $-2.11 /-2.32$ & $-2.18 /-2.29$ & $-2.52 /-2.62$ & $-2.37 /-2.41$ & III \\
\hline J04400800+2605253 & IRAS $04370+2559$ & $\mathrm{~K} 6-\mathrm{M} 3.5^{\mathrm{c}}$ & $-0.64 /-1.27$ & $-0.41 /-0.75$ & $-0.52 /-0.81$ & $-0.30 /-0.42$ & II \\
\hline J04403979+2519061 & & M5.25 & $-2.32 /-2.42$ & $-1.83 /-1.88$ & $-2.61 /-2.65$ & $-1.82 /-1.84$ & II \\
\hline J04404950+2551191 & JH 223 & M2 & $-1.51 /-1.58$ & $-1.21 /-1.25$ & $-1.52 /-1.55$ & $-1.14 /-1.16$ & II \\
\hline J04410424+2557561 & Haro 6-32 & M5 & $-2.45 /-2.50$ & $-2.74 /-2.77$ & $-2.73 /-2.76$ & $-2.93 /-2.94$ & III \\
\hline J04410470+2451062 & IW Tau A+B & K7 & $-2.60 /-2.66$ & $-2.74 /-2.77$ & $-2.75 /-2.77$ & $-2.84 /-2.85$ & III \\
\hline J04410826+2556074 & ITG 33A & M3 & $-0.39 /-0.78$ & $-0.40 /-0.60$ & $-0.61 /-0.78$ & $-0.49 /-0.56$ & II \\
\hline J04411078+2555116 & ITG 34 & M5.5 & $-1.17 /-1.27$ & $-0.98 /-1.04$ & $-1.04 /-1.08$ & $-0.88 /-0.90$ & II \\
\hline J04411267+2546354 & IRAS $04381+2540$ & $\mathrm{~K} 6-\mathrm{M} 3.5^{\mathrm{c}}$ & 1.37 & 1.05 & 1.07 & 0.84 & I \\
\hline J04411681+2840000 & $\mathrm{CoKu} \mathrm{Tau/4}$ & M1.5 & $-2.42 /-2.58$ & $-0.43 /-0.52$ & $-2.46 /-2.54$ & $0.06 / 0.03$ & II \\
\hline J04412464+2543530 & ITG 40 & M3.5 & $-0.72 /-1.84$ & $-0.51 /-1.11$ & $-1.06 /-1.58$ & $-0.60 /-0.81$ & II \\
\hline J04413882+2556267 & IRAS $04385+2550$ & M0 & $-0.46 /-0.83$ & $-0.05 /-0.25$ & $-0.27 /-0.45$ & $0.14 / 0.07$ & II \\
\hline J04414489+2301513 & $\ldots$ & M8.5 & $-1.24 /-1.28$ & $\ldots$ & $-1.40 /-1.41$ & $\ldots$ & II \\
\hline J04414565+2301580 & $\ldots$ & M4.5 & $-2.33 /-2.35$ & $\ldots$ & $\ldots$ & $\ldots$ & III \\
\hline J04414825+2534304 & $\ldots$ & M7.75 & $-0.88 /-0.99$ & $-0.60 /-0.66$ & $-0.76 /-0.81$ & $-0.47 /-0.49$ & II \\
\hline J04420548+2522562 & LkHa $332 / G 2 ~ A+B$ & M0 & $-2.41 /-2.59$ & $-2.48 /-2.57$ & $-2.59 /-2.67$ & $-2.57 /-2.61$ & III \\
\hline J04420732+2523032 & LkHa $332 / G 1 A+B$ & M1 & $-2.45 /-2.65$ & & $-2.67 /-2.76$ & & III \\
\hline J04420777+2523118 & V955 Tau A+B & K7 & $-0.92 /-1.16$ & $-0.90 /-1.02$ & $-1.03 /-1.14$ & $-0.94 /-0.98$ & II \\
\hline J04422101+2520343 & CIDA 7 & M4.75 & $-1.09 /-1.13$ & $-0.59 /-0.62$ & $-0.88 /-0.90$ & $-0.38 /-0.39$ & II \\
\hline J04423769+2515374 & DP Tau & M0.5 & $-0.34 /-0.55$ & $-0.21 /-0.33$ & $-0.32 /-0.41$ & $-0.17 /-0.21$ & II \\
\hline J04430309+2520187 & GO Tau & M0 & $-1.41 /-1.49$ & $-0.96 /-1.00$ & $-1.01 /-1.05$ & $-0.68 /-0.69$ & II \\
\hline J04432023+2940060 & CIDA 14 & M5 & $-1.49 /-1.52$ & $-1.40 /-1.41$ & $-1.32 /-1.34$ & $-1.30 /-1.31$ & II \\
\hline J04442713+2512164 & IRAS $04414+2506$ & M7.25 & $-0.61 /-0.70$ & $-0.38 /-0.43$ & $-0.63 /-0.67$ & $-0.33 /-0.35$ & II \\
\hline J04455129+1555496 & HD 30171 & G5 & $-2.72 /-2.75$ & $\cdots$ & $-2.80 /-2.81$ & & III \\
\hline J04455134+1555367 & IRAS $04429+1550$ & M2.5 & $-1.09 /-1.16$ & $-0.35 /-0.39$ & $-0.76 /-0.79$ & $-0.03 /-0.04$ & II \\
\hline J04464260+2459034 & RXJ 04467+2459 & M4 & $-2.48 /-2.48$ & $\ldots$ & $-2.71 /-2.71$ & $\ldots$ & III \\
\hline J04465305+1700001 & DQ Tau & M0 & $-0.79 /-0.90$ & $\ldots$ & $-0.70 /-0.76$ & $\ldots$ & II \\
\hline J04465897+1702381 & Haro 6-37 A & $\mathrm{K} 7$ & $-1.11 /-1.35$ & $\ldots$ & $-1.23 /-1.34$ & $\cdots$ & II \\
\hline J04465897+1702381 & Haro 6-37 B & M1 & -0.85 & $\cdots$ & -0.70 & $\cdots$ & II \\
\hline J04470620+1658428 & DR Tau & K5 & $-0.58 /-0.74$ & $\cdots$ & $\ldots$ & $\cdots$ & II \\
\hline J04474859+2925112 & DS Tau & K5 & $-1.26 /-1.38$ & $\cdots$ & $-1.17 /-1.23$ & $\cdots$ & II \\
\hline J04484189+1703374 & & M7 & $-2.36 /-2.36$ & $\ldots$ & $-2.65 /-2.65$ & $\ldots$ & III \\
\hline J04514737+3047134 & UY Aur A+B & M0 & $-0.41 /-0.58$ & $\cdots$ & $-0.36 /-0.44$ & $\cdots$ & II \\
\hline J04520668+3047175 & IRAS $04489+3042$ & M4 & $0.24 /-0.49$ & $\ldots$ & $-0.25 /-0.59$ & $\ldots$ & I \\
\hline J04551098+3021595 & GM Aur & K7 & $-1.92 /-1.94$ & $-0.64 /-0.65$ & $-1.73 /-1.74$ & $-0.24 /-0.24$ & II \\
\hline J04552333+3027366 & & M6.25 & $-2.33 /-2.39$ & $\cdots$ & $-2.65 /-2.68$ & & III \\
\hline J04553695+3017553 & LkCa 19 & K0 & $-2.72 /-2.78$ & $-2.46 /-2.49$ & $-2.77 /-2.79$ & $-2.41 /-2.42$ & III \\
\hline J04554046+3039057 & $\ldots$ & M5.25 & $-2.41 /-2.42$ & $\ldots$ & $-2.61 /-2.62$ & & III \\
\hline J04554535+3019389 & $\ldots$ & M4.75 & $-1.54 /-1.54$ & $-1.33 /-1.33$ & $-1.43 /-1.43$ & $-1.23 /-1.23$ & II \\
\hline J04554582+3033043 & AB Aur & B9 & & $\ldots$ & & $\ldots$ & II \\
\hline J04554757+3028077 & $\ldots$ & M4.75 & $-2.40 /-2.40$ & $\ldots$ & $-2.64 /-2.64$ & & III \\
\hline J04554801+3028050 & $\ldots$ & M5.6 & $-1.28 /-1.28$ & $-0.91 /-0.91$ & $-1.28 /-1.28$ & $-0.82 /-0.82$ & II \\
\hline J04554820+3030160 & XEST 26-052 & M4.5 & $-2.44 /-2.44$ & $\ldots$ & $-2.63 /-2.63$ & $\ldots$ & III \\
\hline J04554969+3019400 & & M6 & $-1.91 /-1.91$ & $-1.43 /-1.43$ & $-2.02 /-2.02$ & $-1.35 /-1.35$ & II \\
\hline J04555288+3006523 & $\cdots$ & M5.25 & $-2.44 /-2.47$ & $\ldots$ & $-2.68 /-2.70$ & $\ldots$ & III \\
\hline J04555605+3036209 & XEST 26-062 & M4 & $-1.29 /-1.37$ & $-0.75 /-0.80$ & $-1.12 /-1.16$ & $-0.54 /-0.56$ & II \\
\hline J04555636+3049374 & $\ldots$ & M5 & $-2.43 /-2.44$ & $\ldots$ & $-2.66 /-2.67$ & $\ldots$ & III \\
\hline J04555938+3034015 & SU Aur & G2 & $-1.20 /-1.33$ & $\ldots$ & & $\ldots$ & II \\
\hline J04560118+3026348 & XEST26-071 & M3.5 & $-1.34 /-1.39$ & $-1.25 /-1.28$ & $-1.24 /-1.27$ & $-1.18 /-1.20$ & II \\
\hline J04560201+3021037 & HBC 427 & K5 & $-2.72 /-2.75$ & $-2.69 /-2.71$ & $-2.77 /-2.79$ & $-2.71 /-2.72$ & III \\
\hline J04574903+3015195 & & M9.25 & $-2.29 /-2.29$ & $\ldots$ & $-2.76 /-2.76$ & $\ldots$ & III \\
\hline J04584626+2950370 & MWC 480 & $\mathrm{~A} 2$ & $\ldots$ & $\ldots$ & $\ldots$ & $\ldots$ & II \\
\hline J05030659+2523197 & V836 Tau & K7 & $-1.51 /-1.55$ & $-1.04 /-1.06$ & $-1.14 /-1.16$ & $-0.76 /-0.77$ & II \\
\hline J05044139+2509544 & CIDA 8 & M3.5 & $-1.63 /-1.74$ & $-1.18 /-1.24$ & $-1.47 /-1.52$ & $-0.99 /-1.01$ & II \\
\hline J05052286+2531312 & CIDA 9 & K8 & 0.19 & $\ldots$ & -0.37 & $\ldots$ & II \\
\hline J05061674+2446102 & CIDA 10 & M4 & $-2.50 /-2.54$ & $\ldots$ & $-2.66 /-2.67$ & $\ldots$ & III \\
\hline J05062332+2432199 & CIDA 11 & M3.5 & $-1.62 /-1.66$ & $\ldots$ & $-1.38 /-1.40$ & $\ldots$ & II \\
\hline J05064662+2104296 & $\cdots$ & M5.25 & $-2.40 /-2.42$ & $\cdots$ & $-2.67 /-2.68$ & $\cdots$ & III \\
\hline J05071206+2437163 & RXJ $05072+2437$ & K6 & $-2.68 /-2.71$ & $\cdots$ & $-2.76 /-2.77$ & $\ldots$ & III \\
\hline J05074953+3024050 & RW Aur A+B & K3 & $-0.85 /-0.96$ & $\ldots$ & $-0.56 /-0.61$ & $\cdots$ & II \\
\hline J05075496+2500156 & CIDA 12 & M4 & $-1.59 /-1.62$ & $\cdots$ & $-1.31 /-1.32$ & $\cdots$ & II \\
\hline
\end{tabular}


TABLE 7 - Continued

\begin{tabular}{|c|c|c|c|c|c|c|c|}
\hline $2 \mathrm{MASS}^{\mathrm{a}}$ & Name & $\begin{array}{c}\text { Spectral } \\
\text { Type }\end{array}$ & $\alpha(2-8 \mu \mathrm{m})$ & $\alpha(2-24 \mu \mathrm{m})$ & $\alpha(3.6-8 \mu \mathrm{m})$ & $\alpha(3.6-24 \mu \mathrm{m})$ & $\begin{array}{l}\text { SED } \\
\text { Class }\end{array}$ \\
\hline
\end{tabular}

Note. - Entries consist of observed and dereddened values, respectively. Only the observed values are listed if extinction estimates are unavailable.

a 2 MASS Point Source Catalog.

b Because this multiple system is unresolved at $24 \mu \mathrm{m}$, this slope was computed with the total flux of the system in the band at shorter wavelengths.

c The spectral type bin in which this source was placed in Table 8 based on its bolometric luminosity.

TABLE 8

Statistics of SED Classes

\begin{tabular}{lrrrrrrr}
\hline \hline & \multicolumn{3}{c}{ All of Taurus } & & \multicolumn{3}{c}{ XEST Fields } \\
\cline { 2 - 3 } \cline { 6 - 8 } Spectral Type & \multicolumn{1}{c}{ I } & \multicolumn{1}{c}{ II } & III & & I & II & III \\
\hline$<$ K6 & 10 & 23 & 8 & & 9 & 14 & 3 \\
K6 and $\leq$ M3.5 & 22 & 90 & 40 & & 19 & 56 & 23 \\
$>$ M3.5 and $<$ M6 & 5 & 44 & 42 & & 2 & 20 & 23 \\
M 6 and $\leq$ M8 & 3 & 18 & 25 & & 1 & 7 & 12 \\
$>$ M8 & 0 & 9 & 11 & & 0 & 3 & 3 \\
Total & 42 & 184 & 126 & & 31 & 100 & 64
\end{tabular}

a Spectral types have been estimated from bolometric luminosities for stars that lack spectral classifications, most of which are class I sources. The spectral type bins in which these sources appear are indicated in Table 7

TABLE 9

IRAC Photometry for Probable Members of $\eta$ Cha

\begin{tabular}{lrrrrc}
\hline \hline $\mathrm{ID}^{\mathrm{a}}$ & \multicolumn{1}{c}{$[3.6]$} & \multicolumn{1}{c}{$[4.5]$} & {$[5.8]$} & {$[8.0]$} & Date \\
\hline 1 & $7.18 \pm 0.02$ & $7.20 \pm 0.02$ & $7.13 \pm 0.03$ & $7.09 \pm 0.03$ & 2004 Jun 10 \\
2 & out & out & $5.63 \pm 0.03$ & out & 2004 Jun 10 \\
3 & $9.28 \pm 0.02$ & $9.23 \pm 0.02$ & $9.11 \pm 0.03$ & $9.18 \pm 0.03$ & 2004 Jun 10 \\
4 & $8.46 \pm 0.02$ & $8.44 \pm 0.02$ & $8.37 \pm 0.03$ & $8.37 \pm 0.03$ & 2004 Jun 10 \\
5 & $9.62 \pm 0.02$ & $9.51 \pm 0.02$ & $9.36 \pm 0.03$ & $8.95 \pm 0.03$ & 2004 Jun 10 \\
6 & $9.19 \pm 0.02$ & $9.11 \pm 0.02$ & $9.09 \pm 0.03$ & $9.04 \pm 0.03$ & 2004 Jun 10 \\
7 & $7.53 \pm 0.02$ & $7.54 \pm 0.02$ & $7.48 \pm 0.03$ & $7.48 \pm 0.03$ & 2004 Jun 10 \\
8 & sat & sat & $5.38 \pm 0.03$ & $5.41 \pm 0.03$ & 2004 Jun 10 \\
9 & $8.98 \pm 0.02$ & $8.79 \pm 0.02$ & $8.55 \pm 0.03$ & $7.99 \pm 0.03$ & 2004 Jun 10 \\
10 & $8.59 \pm 0.02$ & $8.60 \pm 0.02$ & $8.53 \pm 0.03$ & $8.52 \pm 0.03$ & 2004 Jun 10 \\
11 & $7.10 \pm 0.02$ & $6.85 \pm 0.02$ & $6.56 \pm 0.03$ & $5.93 \pm 0.03$ & 2004 Jun 10 \\
12 & $8.19 \pm 0.02$ & $8.15 \pm 0.02$ & $8.10 \pm 0.03$ & $8.10 \pm 0.03$ & 2004 Jun 10 \\
13 & $6.98 \pm 0.02$ & $6.96 \pm 0.02$ & $6.95 \pm 0.03$ & $6.94 \pm 0.03$ & 2004 Jun 10 \\
14 & $10.53 \pm 0.02$ & $10.28 \pm 0.02$ & $10.04 \pm 0.03$ & $9.51 \pm 0.03$ & 2004 Jun 10 \\
15 & $8.40 \pm 0.02$ & $7.90 \pm 0.02$ & $7.41 \pm 0.03$ & $6.54 \pm 0.03$ & 2004 Jun 10 \\
16 & $11.02 \pm 0.02$ & $10.74 \pm 0.02$ & $10.44 \pm 0.03$ & $9.79 \pm 0.03$ & 2004 Jun 10 \\
17 & $10.09 \pm 0.02$ & $9.97 \pm 0.02$ & $9.93 \pm 0.03$ & $9.92 \pm 0.03$ & 2004 Jun 10 \\
18 & $10.57 \pm 0.02$ & $10.44 \pm 0.02$ & $10.41 \pm 0.03$ & $10.35 \pm 0.03$ & 2004 Jun 10
\end{tabular}

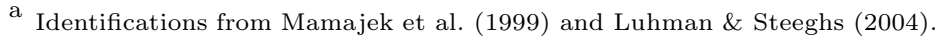

TABLE 10

MiPS Photometry for Probable Members of $\eta$ Cha

\begin{tabular}{lcl}
\hline \hline ID $^{\mathrm{a}}$ & {$[24]$} & \multicolumn{1}{c}{ Date } \\
\hline 1 & $7.07 \pm 0.05$ & 2005 Mar 9 \\
& $7.01 \pm 0.04$ & 2005 Apr 8 \\
2 & $4.37 \pm 0.04$ & 2004 Feb 21 \\
& $4.40 \pm 0.04$ & 2005 Apr 8 \\
3 & $8.68 \pm 0.05$ & 2005 Mar 9 \\
& $8.65 \pm 0.07$ & 2005 Apr 8 \\
4 & $7.62 \pm 0.05$ & 2005 Mar 8 \\
& $7.73 \pm 0.04$ & 2005 Apr 8 \\
& $7.62 \pm 0.04$ & 2008 May 18 \\
5 & $5.18 \pm 0.04$ & 2004 Feb 21 \\
& $5.10 \pm 0.04$ & 2005 Apr 8 \\
6 & $8.84 \pm 0.05$ & 2005 Apr 8 \\
7 & $7.47 \pm 0.05$ & 2005 Mar 8 \\
& $7.39 \pm 0.04$ & 2005 Apr 8 \\
& $7.36 \pm 0.04$ & 2008 May 18 \\
\hline
\end{tabular}


TABLE $10-$ Continued

\begin{tabular}{lcl}
\hline \hline ID $^{\mathrm{a}}$ & {$[24]$} & Date \\
\hline 8 & $5.50 \pm 0.04$ & 2005 Mar 8 \\
& $5.39 \pm 0.04$ & 2005 Apr 8 \\
& $5.41 \pm 0.04$ & 2008 May 18 \\
9 & $5.40 \pm 0.04$ & 2005 Apr 8 \\
10 & $8.40 \pm 0.05$ & 2005 Apr 8 \\
11 & $3.81 \pm 0.05$ & 2004 Feb 21 \\
& $3.61 \pm 0.04$ & 2004 Sep 18 \\
& $3.75 \pm 0.04$ & 2005 Apr 8 \\
& $3.68 \pm 0.04$ & 2008 May 18 \\
12 & $7.95 \pm 0.05$ & 2004 Jun 22 \\
& $7.91 \pm 0.04$ & 2005 Apr 8 \\
13 & $6.99 \pm 0.04$ & 2005 Mar 9 \\
& $7.00 \pm 0.04$ & 2005 Apr 8 \\
14 & $7.18 \pm 0.04$ & 2005 Apr 8 \\
15 & $3.40 \pm 0.04$ & 2005 Mar 8 \\
& $3.53 \pm 0.04$ & 2005 Apr 8 \\
& $3.47 \pm 0.04$ & 2008 May 18 \\
16 & $7.17 \pm 0.04$ & 2005 Apr 8 \\
17 & $9.81 \pm 0.17$ & 2008 May 18 \\
18 & out & . \\
& & \\
\hline
\end{tabular}

${ }^{\text {a }}$ Identifications from Mamajek et al. (1999) and Luhman \& Steeghs (2004).

TABLE 11

MiPs Photometry for Probable Members of $\epsilon$ Cha

\begin{tabular}{lrc}
\hline \hline \multicolumn{1}{c}{ ID $^{\mathrm{a}}$} & \multicolumn{1}{c}{$[24]$} & \multicolumn{2}{c}{ Date } \\
\hline 1 & $\ldots$ & $\ldots$ \\
2 & $4.99 \pm 0.04$ & 2006 Feb 22 \\
$3+4+5+6$ & sat & $\ldots$ \\
7 & $2.1 \pm 0.2$ & 2006 Feb 22 \\
8 & $6.69 \pm 0.04$ & 2006 Feb 27 \\
9 & $9.9 \pm 0.2$ & 2006 Feb 27 \\
10 & $6.68 \pm 0.04$ & 2006 Feb 27 \\
11 & $5.30 \pm 0.04$ & 2006 Feb 27 \\
12 & $9.8 \pm 0.2$ & 2006 Feb 27 \\
& & \\
\hline
\end{tabular}

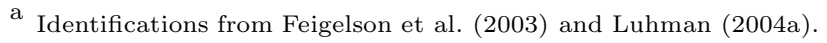

TABLE 12

Spitzer Photometry for Probable Members of TWA

\begin{tabular}{|c|c|c|c|c|c|c|c|}
\hline TWA & {$[3.6]$} & {$[4.5]$} & {$[5.8]$} & [8.0] & Date & {$[24]$} & Date \\
\hline 1 & $7.14 \pm 0.02$ & $7.04 \pm 0.02$ & $6.90 \pm 0.03$ & $5.98 \pm 0.03$ & 2003 Dec 20 & $1.24 \pm 0.04$ & 2004 Feb 2 \\
\hline 2 & out & out & out & out & & $6.37 \pm 0.04$ & 2004 Jan 30 \\
\hline 3 & $6.49 \pm 0.02$ & $6.37 \pm 0.02$ & $6.15 \pm 0.03$ & $5.15 \pm 0.03$ & 2004 May 27 & $1.62 \pm 0.04$ & 2004 Feb 2 \\
\hline 4 & $5.59 \pm 0.02$ & $5.54 \pm 0.02$ & $5.43 \pm 0.03$ & $4.79 \pm 0.03$ & 2003 Dec 20 & sat & $\ldots$ \\
\hline 5 & out & out & out & out & $\ldots$ & $6.35 \pm 0.04$ & 2004 Feb 2 \\
\hline 6 & out & out & out & out & $\ldots$ & $7.66 \pm 0.04$ & 2004 May 9 \\
\hline 7 & out & out & out & out & $\cdots$ & $5.97 \pm 0.04$ & $2004 \operatorname{Jan} 30$ \\
\hline $8 \mathrm{~A}$ & out & out & out & out & $\cdots$ & $6.98 \pm 0.04$ & 2004 Jan 31 \\
\hline $8 \mathrm{~B}$ & out & out & out & out & $\ldots$ & $8.17 \pm 0.05$ & 2004 Jan 31 \\
\hline 9 & out & out & out & out & $\ldots$ & $7.21 \pm 0.04$ & 2004 Feb 2 \\
\hline 10 & out & out & out & out & $\ldots$ & $7.75 \pm 0.04$ & 2004 Feb 20 \\
\hline $11 \mathrm{~A}+\mathrm{B}$ & out & out & out & out & $\ldots$ & $0.97 \pm 0.04$ & 2004 Jan 31 \\
\hline $11 \mathrm{C}$ & out & $8.61 \pm 0.02$ & out & $8.52 \pm 0.03$ & 2007 Aug 7 & $8.23 \pm 0.08$ & 2004 Jan 31 \\
\hline 13 & out & out & out & out & $\ldots$ & $6.55 \pm 0.05$ & 2004 Jun 21 \\
\hline 14 & out & out & out & out & $\ldots$ & $8.11 \pm 0.05$ & 2004 Feb 20 \\
\hline 15 & out & out & out & out & $\ldots$ & $8.41 \pm 0.06$ & 2004 Feb 20 \\
\hline 16 & out & out & out & out & $\ldots$ & $7.84 \pm 0.05$ & 2004 Feb 20 \\
\hline 20 & out & out & out & out & $\ldots$ & $8.01 \pm 0.05$ & 2006 Feb 20 \\
\hline 21 & out & out & out & out & $\ldots$ & $7.09 \pm 0.04$ & 2005 Jan 31,2006 Feb 27 \\
\hline 23 & out & out & out & out & $\ldots$ & $7.35 \pm 0.04$ & 2005 Feb 1 \\
\hline 25 & out & out & out & out & $\ldots$ & $7.07 \pm 0.05$ & 2005 Jun 26 \\
\hline 26 & $10.93 \pm 0.02$ & $10.81 \pm 0.02$ & $10.71 \pm 0.03$ & $10.61 \pm 0.03$ & 2005 Jun 10 & $10.31 \pm 0.07$ & 2005 Feb 1 \\
\hline 27 & $11.30 \pm 0.02$ & $10.98 \pm 0.02$ & $10.70 \pm 0.03$ & $10.18 \pm 0.03$ & 2005 Jun 14 & $8.10 \pm 0.04$ & 2005 Jan 27 \\
\hline 28 & $11.19 \pm 0.02$ & $10.80 \pm 0.02$ & $10.45 \pm 0.03$ & $9.95 \pm 0.03$ & 2007 Jul 4 & $8.09 \pm 0.04$ & 2007 Jul 12 \\
\hline 29 & $12.72 \pm 0.02$ & $12.64 \pm 0.02$ & $12.56 \pm 0.03$ & $12.51 \pm 0.03$ & 2009 Mar 19 & out & $\ldots$ \\
\hline
\end{tabular}


TABLE 13 - Continued

\begin{tabular}{llllllll}
\hline \hline Spectral Type & $J-H$ & $H-K_{s}$ & $K_{s}-[3.6]$ & {$[3.6]-[4.5]$} & {$[4.5]-[5.8]$} & {$[5.8]-[8.0]$} & {$[8.0]-[24]$} \\
\hline
\end{tabular}

TABLE 13

Colors of Young Stellar Photospheres

\begin{tabular}{lccccccc}
\hline \hline Spectral Type & $J-H$ & $H-K_{s}$ & $K_{s}-[3.6]$ & {$[3.6]-[4.5]$} & {$[4.5]-[5.8]$} & {$[5.8]-[8.0]$} & {$[8.0]-[24]$} \\
\hline K4 & 0.53 & 0.10 & 0.06 & 0.00 & 0.04 & 0.00 & 0.04 \\
K5 & 0.61 & 0.12 & 0.08 & 0.00 & 0.04 & 0.00 & 0.06 \\
K6 & 0.66 & 0.14 & 0.10 & 0.00 & 0.04 & 0.00 & 0.08 \\
K7 & 0.68 & 0.15 & 0.10 & 0.00 & 0.04 & 0.00 & 0.11 \\
M0 & 0.70 & 0.16 & 0.10 & 0.00 & 0.04 & 0.00 & 0.13 \\
M1 & 0.71 & 0.18 & 0.11 & 0.01 & 0.04 & 0.00 & 0.15 \\
M2 & 0.70 & 0.20 & 0.14 & 0.03 & 0.04 & 0.00 & 0.17 \\
M3 & 0.68 & 0.22 & 0.17 & 0.05 & 0.04 & 0.00 & 0.19 \\
M4 & 0.63 & 0.26 & 0.20 & 0.07 & 0.04 & 0.00 & 0.21 \\
M5 & 0.56 & 0.29 & 0.28 & 0.09 & 0.04 & 0.00 & 0.23 \\
M6 & 0.55 & 0.33 & 0.39 & 0.11 & 0.04 & 0.00 & 0.25 \\
M7 & 0.59 & 0.37 & 0.42 & 0.12 & 0.05 & 0.00 & 0.27 \\
M8 & 0.65 & 0.44 & 0.52 & 0.12 & 0.07 & 0.03 & 0.29 \\
M9 & 0.72 & 0.55 & 0.63 & 0.14 & 0.13 & 0.12 & 0.31 \\
L0 & 0.98 & 0.66 & 0.72 & 0.16 & 0.22 & 0.25 & 0.33 \\
& & & & & & & \\
\hline
\end{tabular}




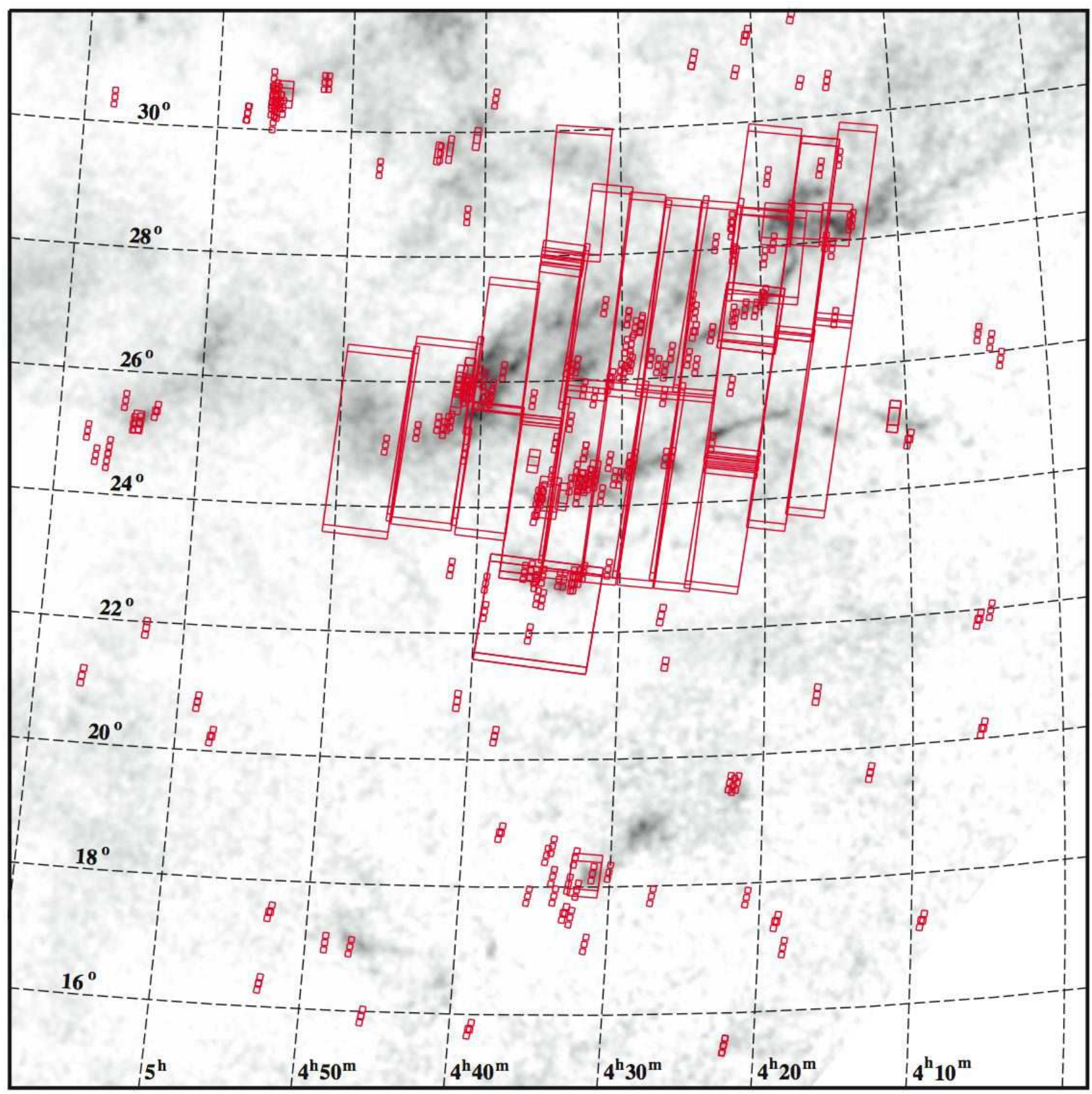

FIG. 1. - Fields in the Taurus star-forming region that have been imaged at 3.6-8.0 $\mu \mathrm{m}$ by IRAC on the Spitzer Space Telescope (Table1). The dark clouds in Taurus are displayed with a map of extinction (grayscale, Dobashi et al. 2005). 


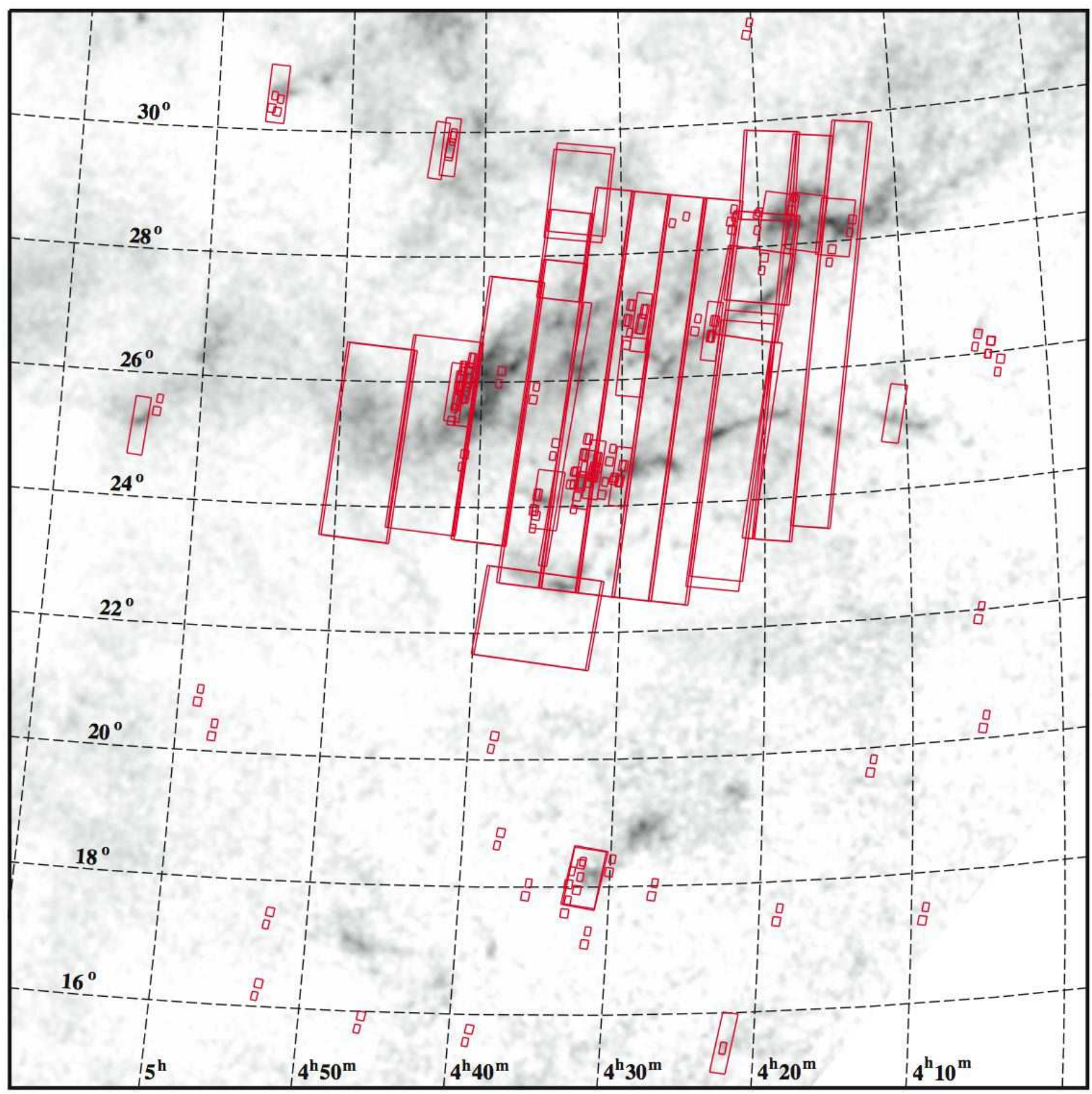

FIG. 2. - Fields in the Taurus star-forming region that have been imaged at $24 \mu \mathrm{m}$ with MIPS on the Spitzer Space Telescope (Table 22). 

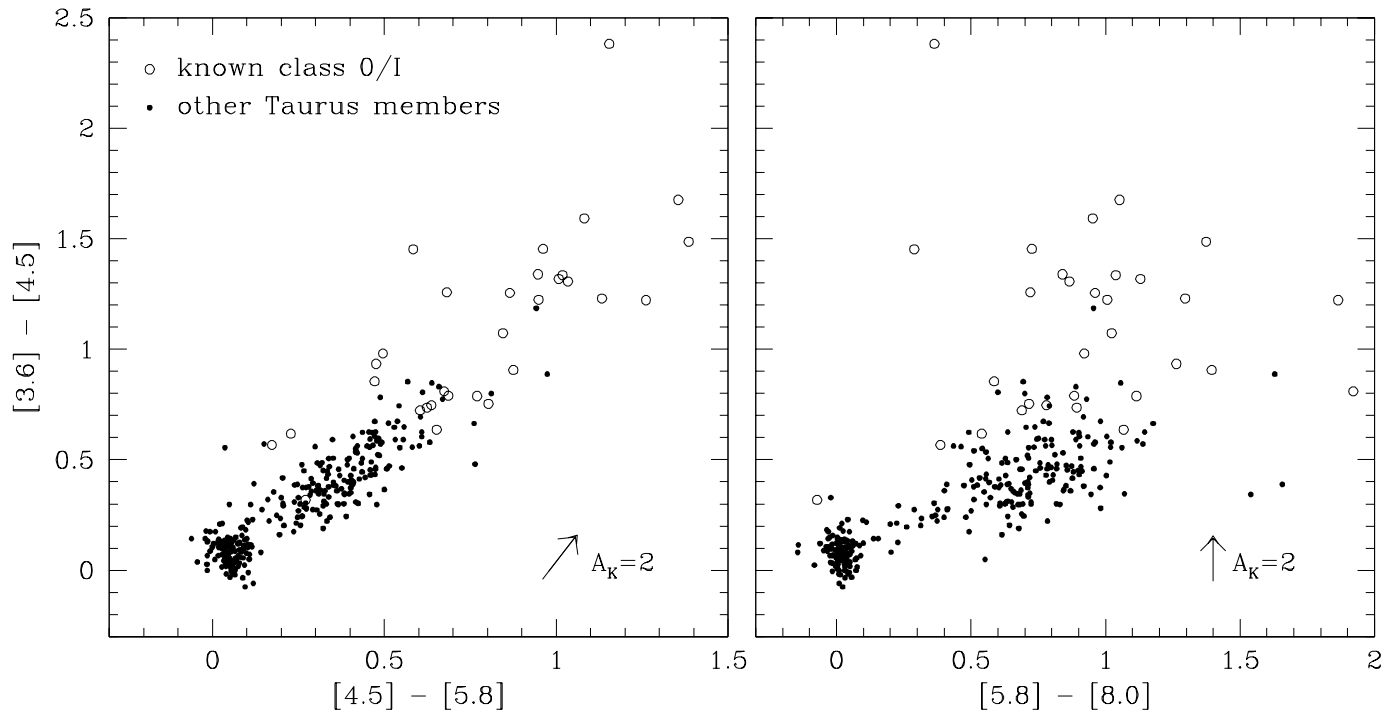

FIG. 3. - Spitzer IRAC color-color diagrams for members of the Taurus star-forming region. Stars that have been previously identified as class 0 and class I sources through mid-IR spectroscopy and other diagnostics are indicated (open circles, $\S$ 3.1). The reddening vectors are based on the extinction law from Flaherty et al. (2007). 

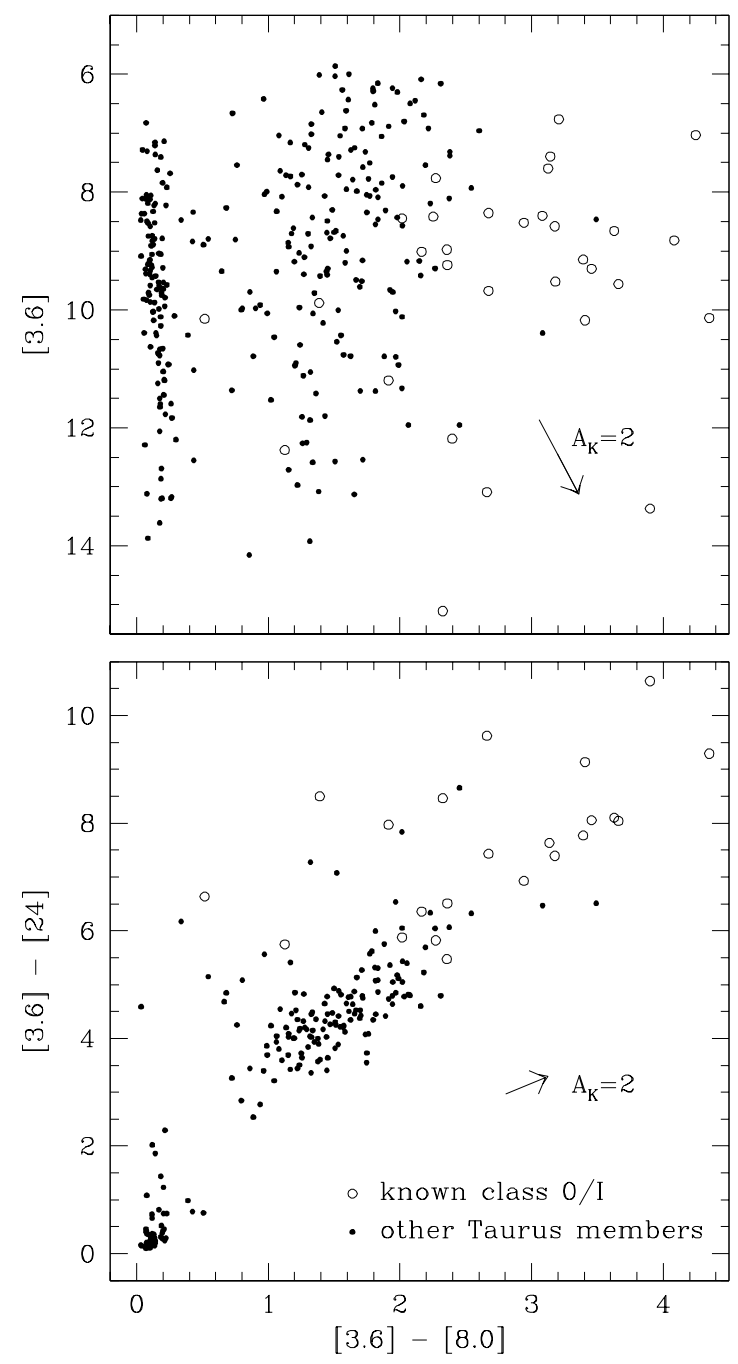

FIG. 4.- Spitzer color-magnitude and color-color diagrams for members of the Taurus star-forming region. Stars that have been previously identified as class 0 and class I sources through mid-IR spectroscopy and other diagnostics are indicated (open circles, $\S 3.1$ ). The reddening vectors are based on the extinction law from Flaherty et al. (2007). 


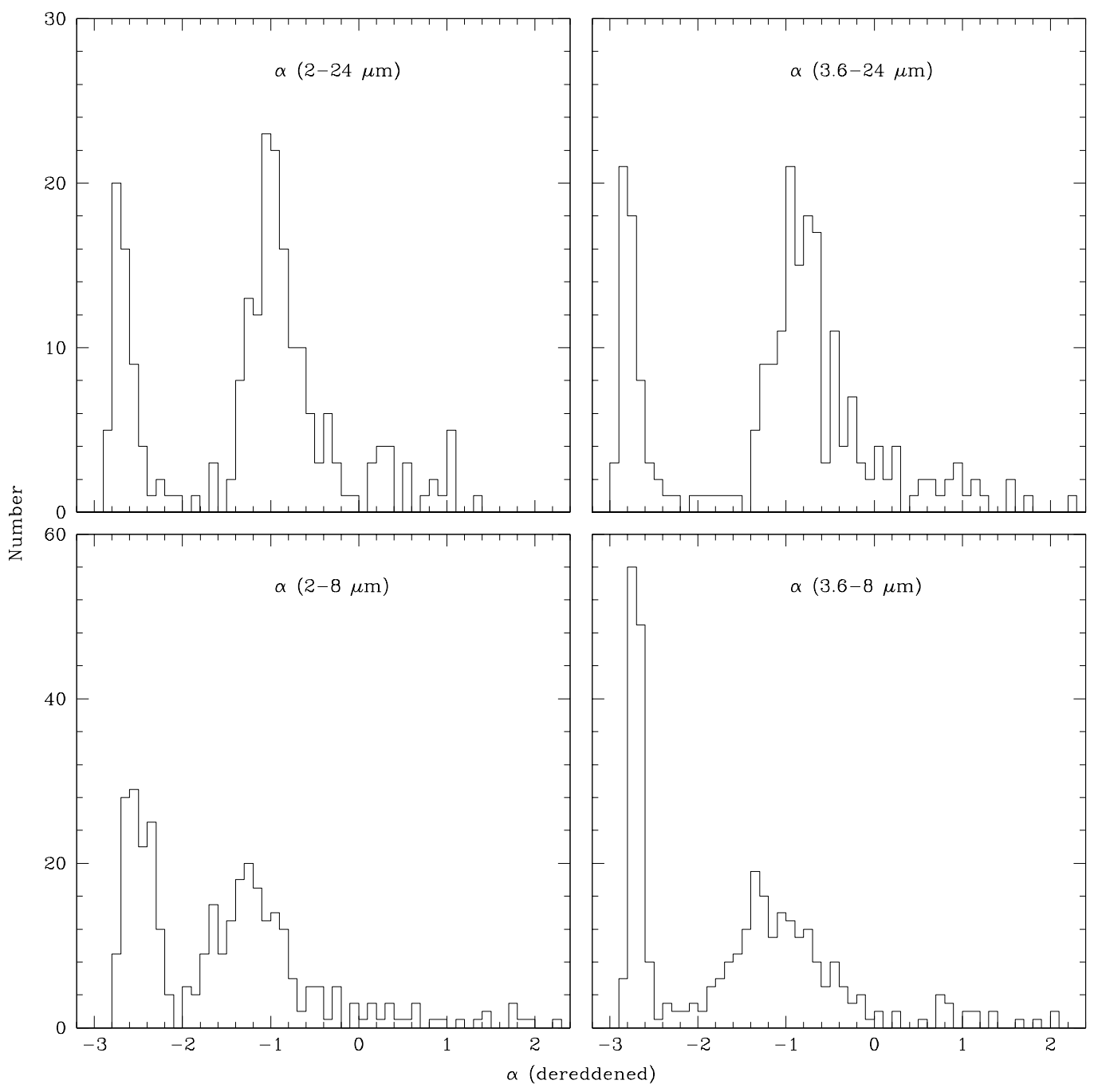

FiG. 5.- Distributions of spectral slopes for members of Taurus (Table 7). 


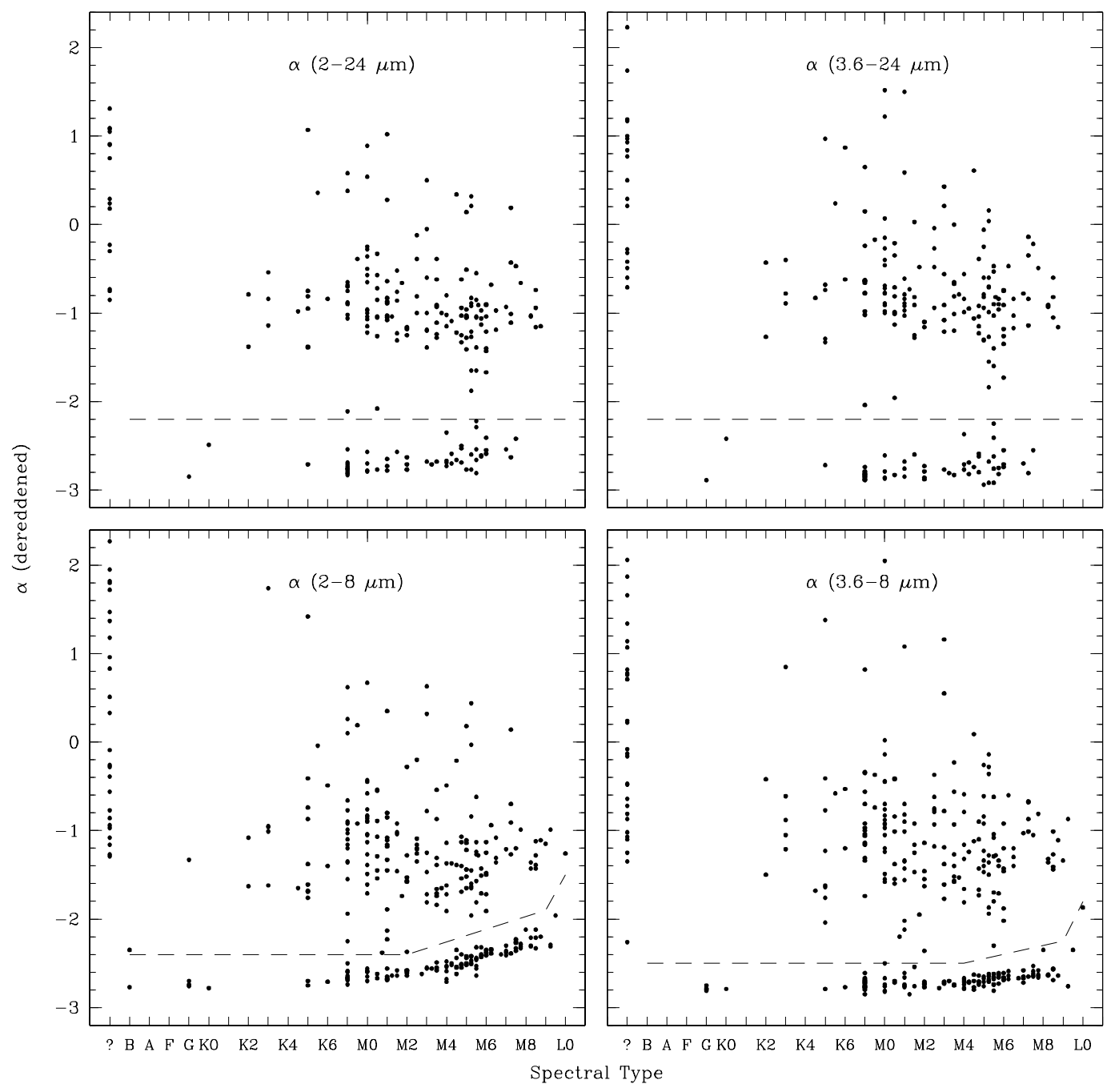

FIG. 6.- Spectral slopes as function of spectral type for members of Taurus (Table 77). Our adopted boundaries for separating classes II and III are indicated (dashed lines). 


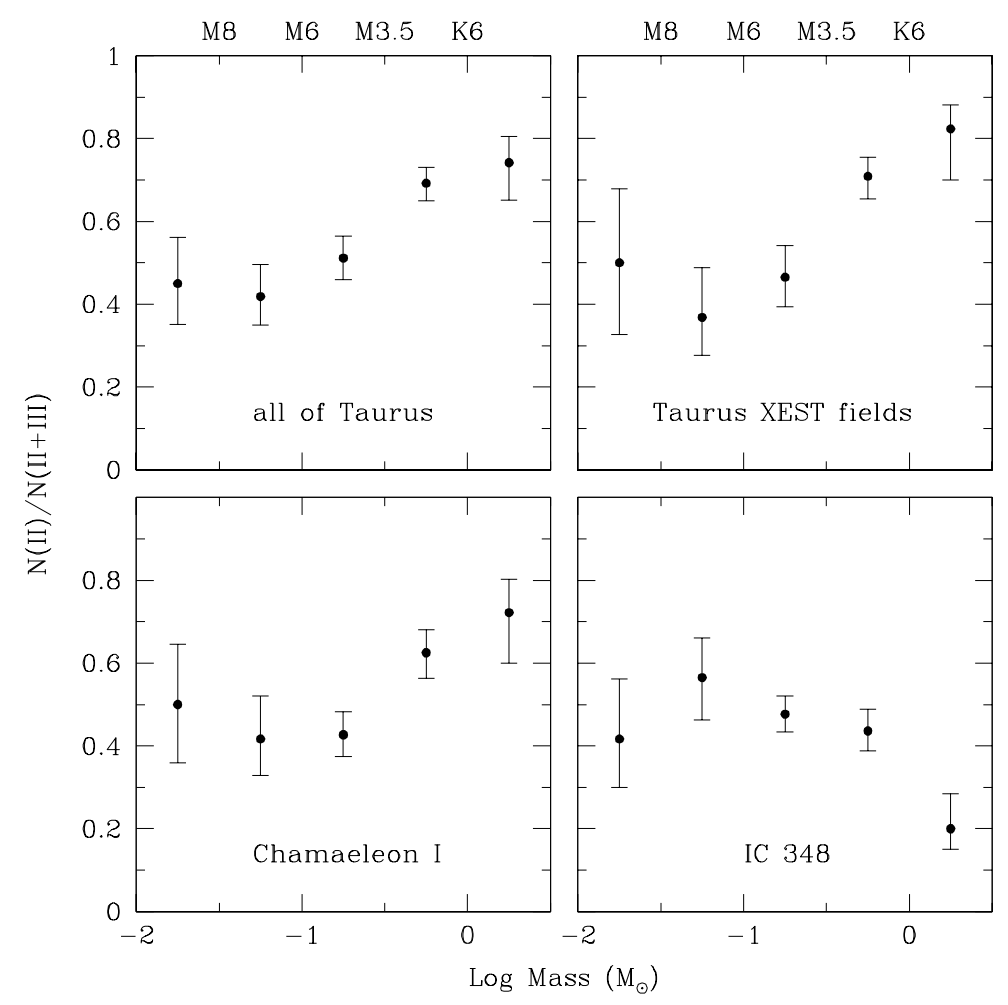

FIG. 7.- Disk fraction as a function of stellar mass and spectral type among all known members of Taurus and the members within the XEST fields. Similar measurements for Chamaeleon I and IC 348 are included for comparison (Lada et al., 2006; Muench et al. 2007; Luhman et al. 2005, 2008a). 


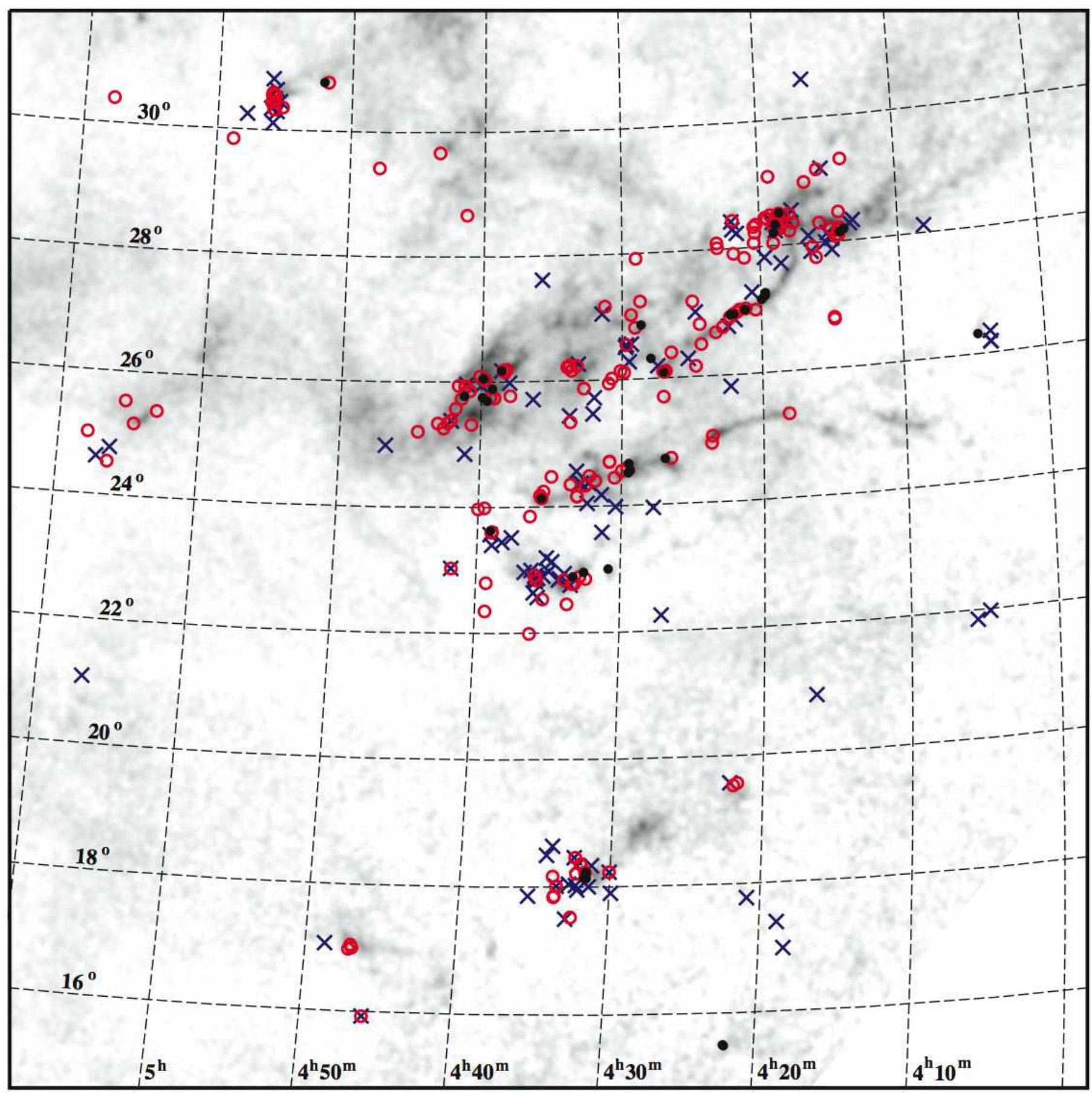

FIG. 8.- Spatial distributions of classes 0/I (black filled circles), II (red open circles), and III (blue crosses) in the Taurus star-forming region. 


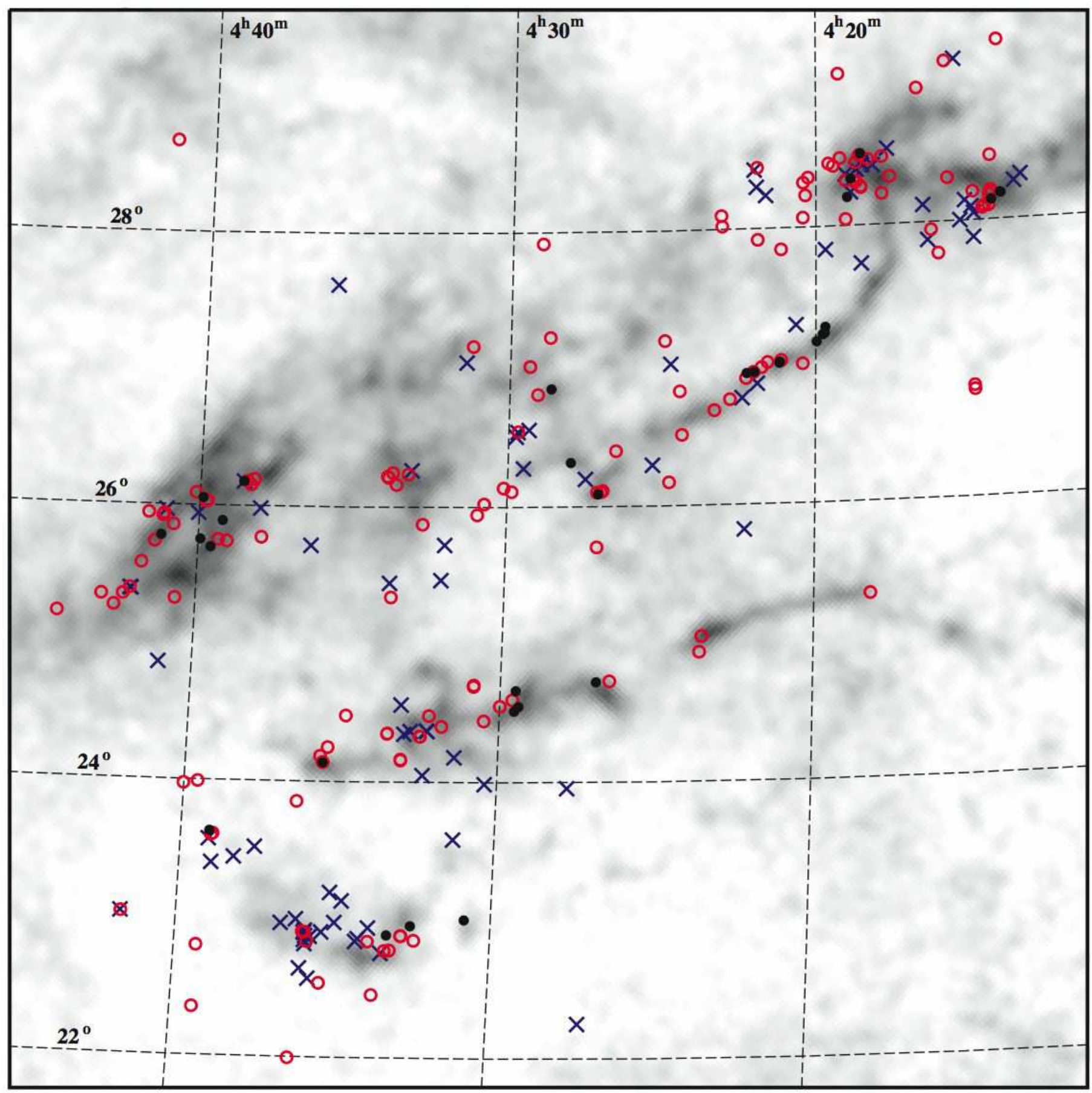

FIG. 9. - Same as Figure 8 but for the central clouds in Taurus. 

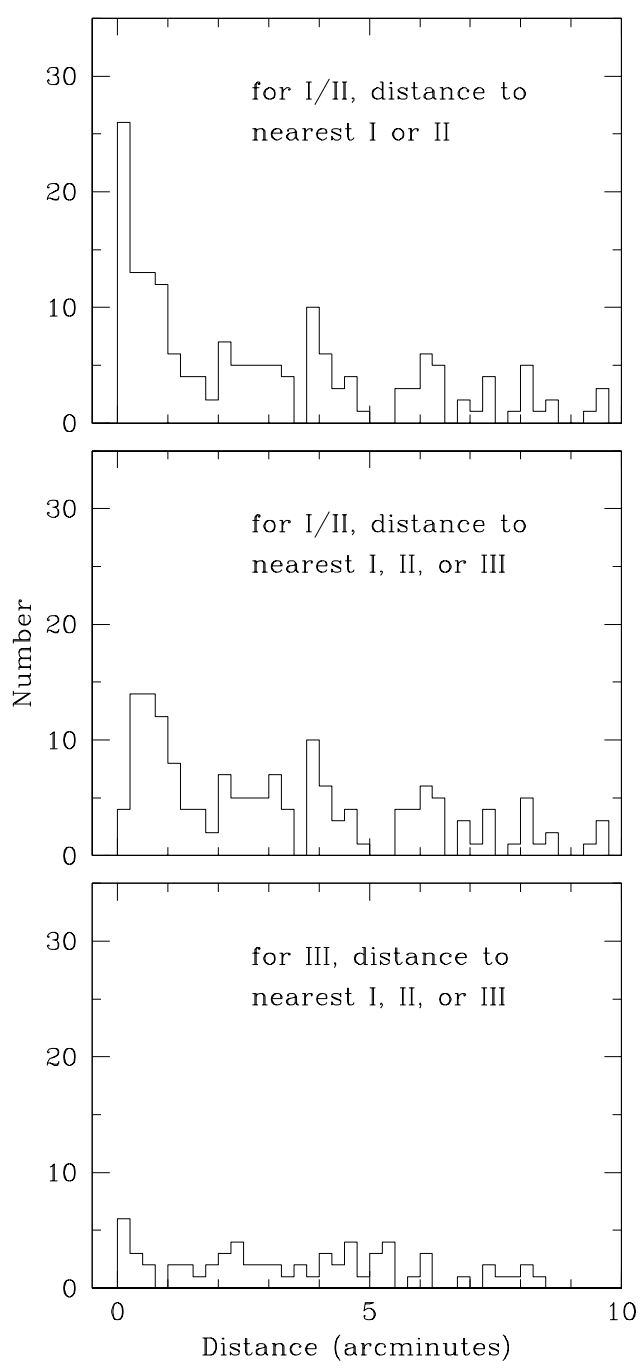

FIG. 10.- Distributions of projected angular distances to the nearest neighbor for different SED classes in Taurus. The bin size is $15^{\prime \prime}$, corresponding to $\sim 0.01 \mathrm{pc}$ at the distance of Taurus. 


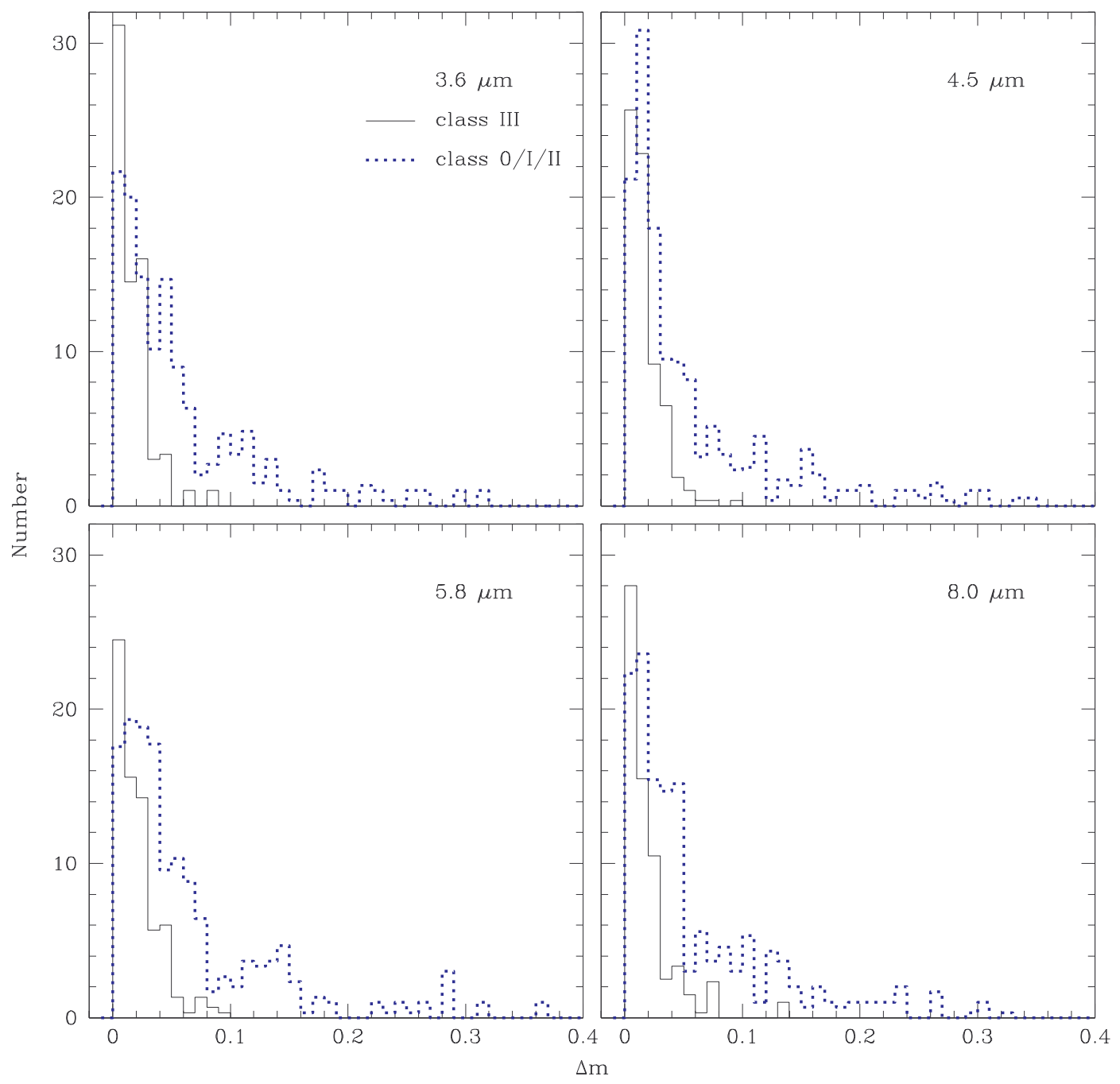

FIG. 11. - Variability of IRAC magnitudes for members of Taurus that have disks (classes 0, I, and II, dotted histograms) and members without disks (class III, solid histograms). The histograms represent distributions of differences between a magnitude and the average magnitude for a given source and band. Each magnitude difference is weighted by the inverse of the number of measurements so that all members contribute equally. 


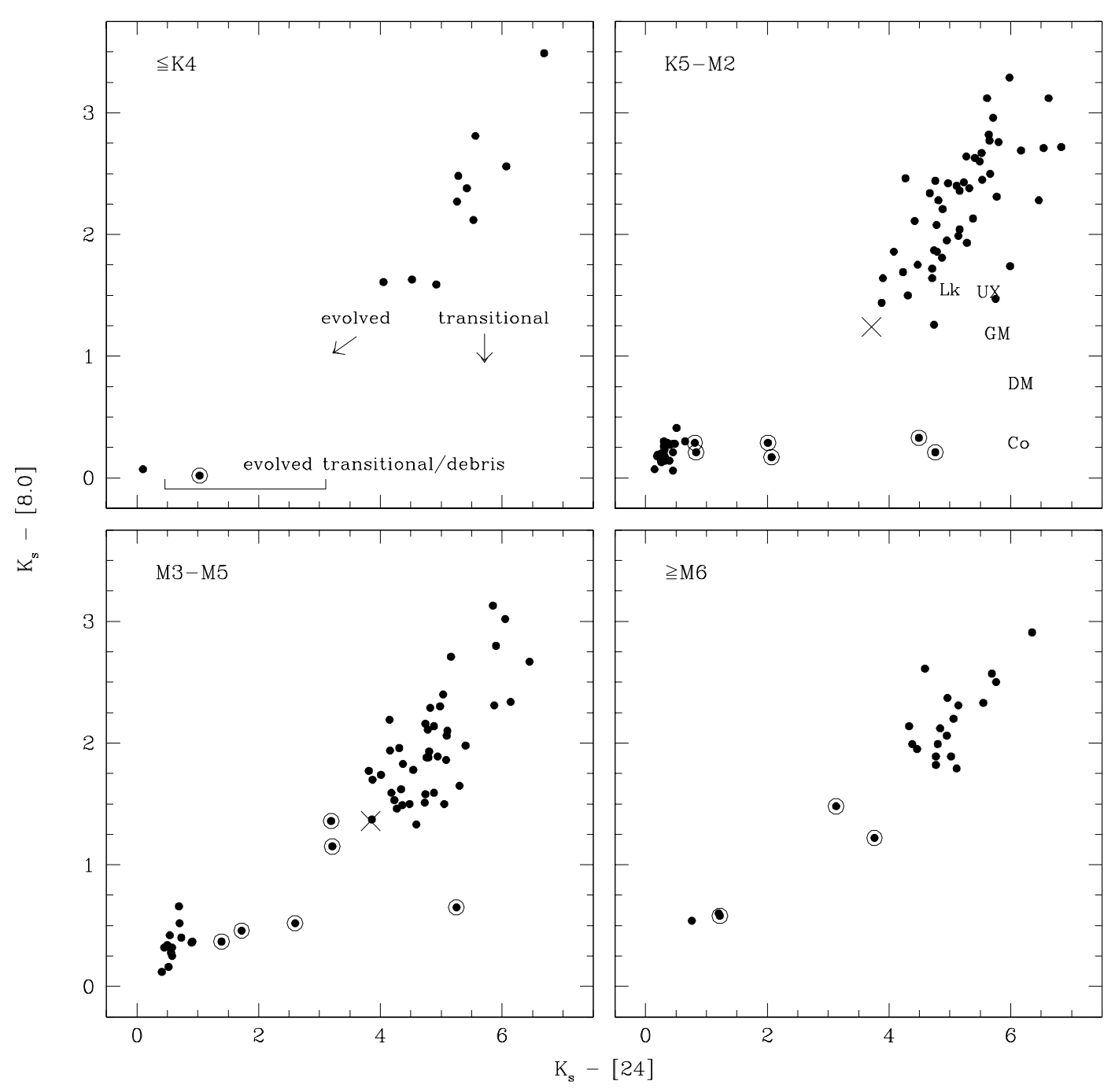

FIG. 12.- $K_{s}-[8.0]$ versus $K_{s}-[24]$ for class II and class III members of Taurus in four ranges of spectral types. The colors predicted by a model of an optically thick disk with a low accretion rate and a high degree of dust settling are shown for stellar masses of 0.2 and $0.7 M_{\odot}(\mathrm{M} 3-\mathrm{M} 5$ and K5-M2, crosses). These models demonstrate that the large, continuous population of red colors can be explained in terms of optically thick disks, which we refer to as primordial disks. In the upper left panel, we indicate the colors expected for optically thick disks that have developed inner holes (transitional), disks that are becoming optically thin throughout their inner regions (evolved), optically thin disks with inner holes (evolved transitional), and disks of second-generation dust (debris). The SEDs of systems that appear to be in these stages according to this diagram (circles) are shown in Figs. 15 16, and 17 For reference, abbreviated names are used as the symbols for the pre-transitional and transitional systems UX Tau A, LkCa 15, GM Aur, DM Tau, and CoKu Tau/4. 


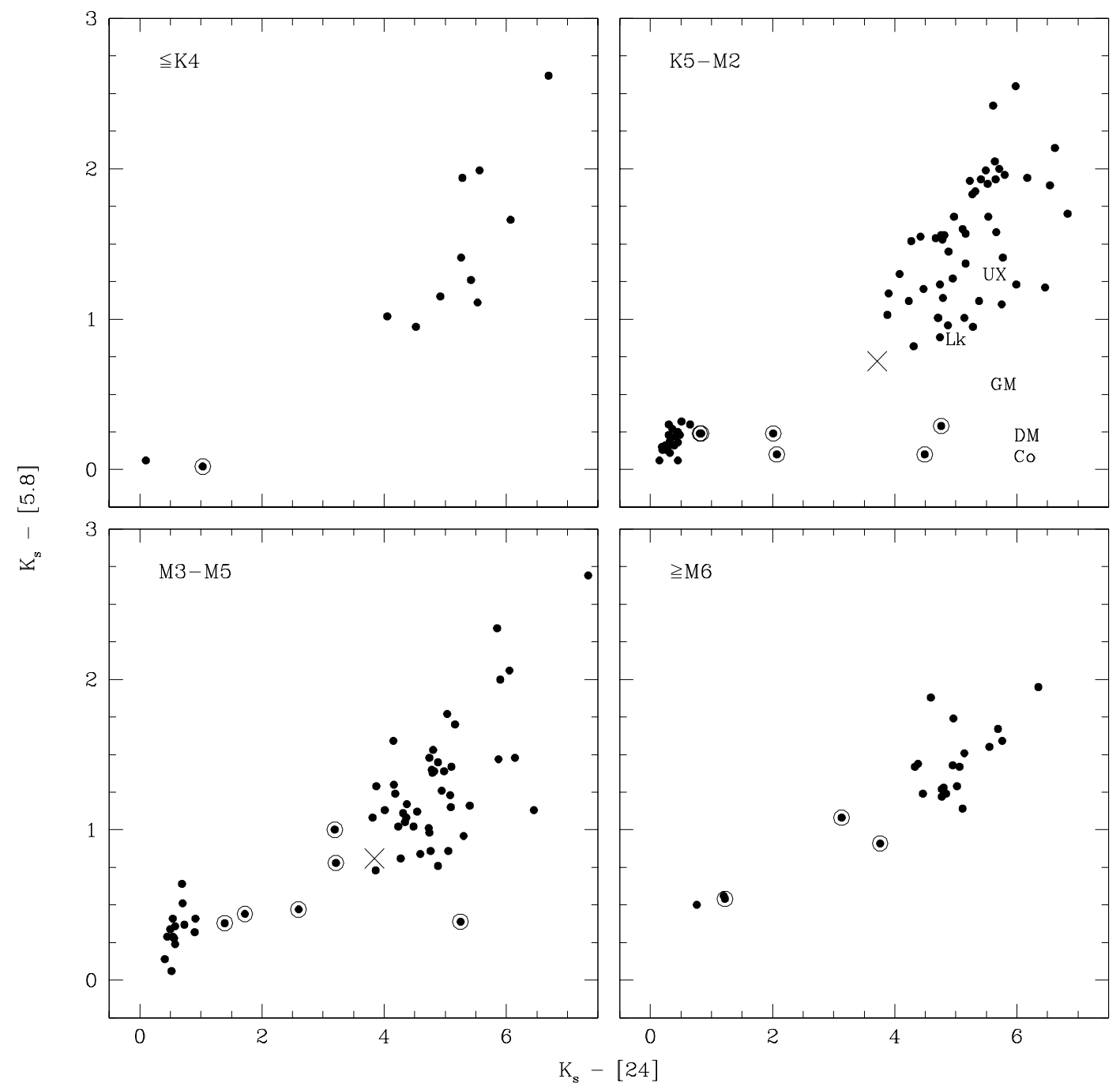

Fig. 13. - Same as Figure 12 but for $K_{s}-[5.8]$ versus $K_{s}-[24]$. 


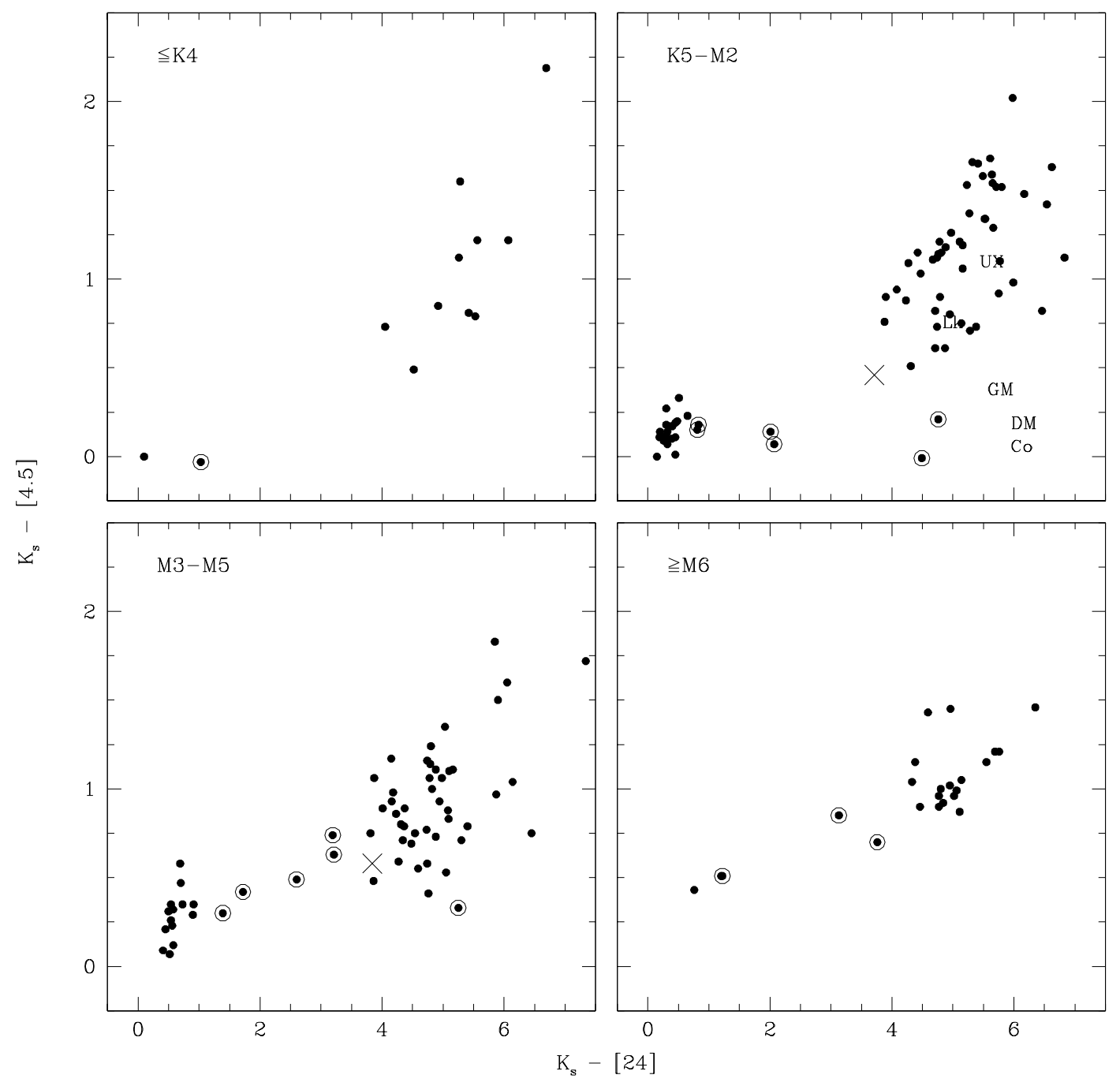

FiG. 14. - Same as Figure 12 but for $K_{s}-[4.5]$ versus $K_{s}-[24]$. 


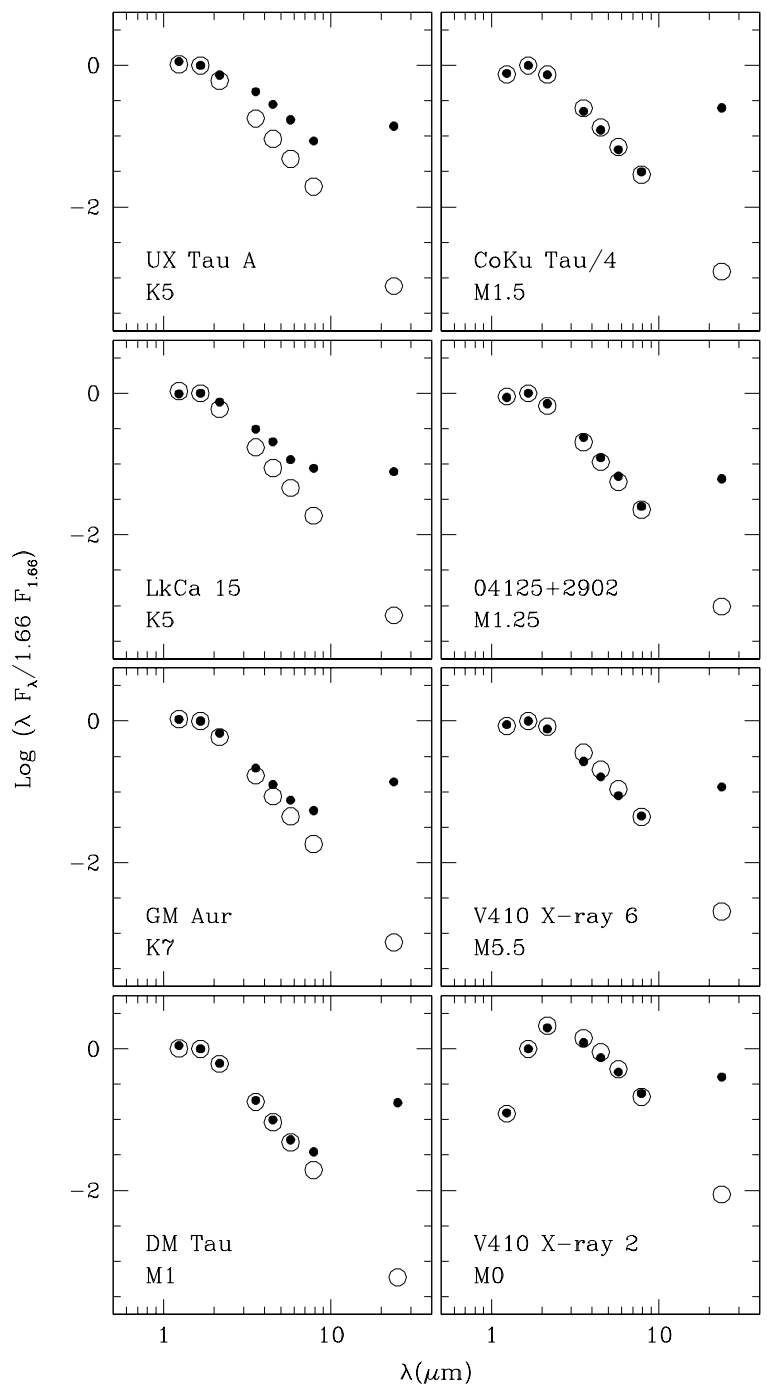

FIG. 15. - SEDs in Taurus that exhibit large $24 \mu \mathrm{m}$ excesses but less excess emission at shorter wavelengths, which indicate the presence of disks with gaps and inner holes (pre-transitional and transitional disks). Each SED is compared to the SED of a stellar photosphere at the same spectral type (circles), which has been reddened by the extinction of the Taurus source and scaled to its $H$-band flux. 


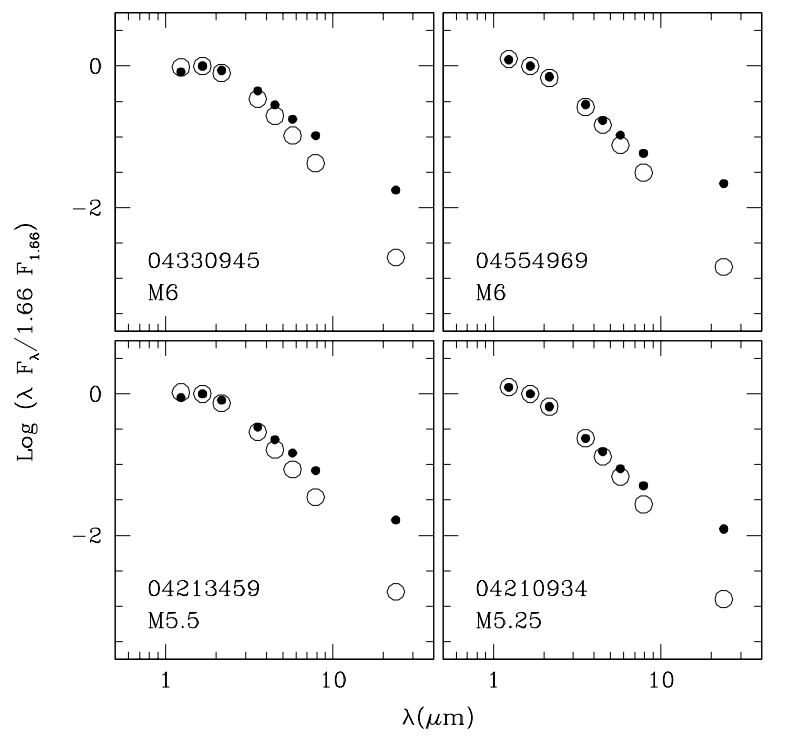

FIG. 16.- SEDs in Taurus that exhibit small excesses at 8 and $24 \mu \mathrm{m}$, which indicate the presence of disks that are becoming optically thin at these wavelengths (evolved disks). Each SED is compared to the SED of a stellar photosphere at the same spectral type (circles), which has been reddened by the extinction of the Taurus source and scaled to its $H$-band flux. 


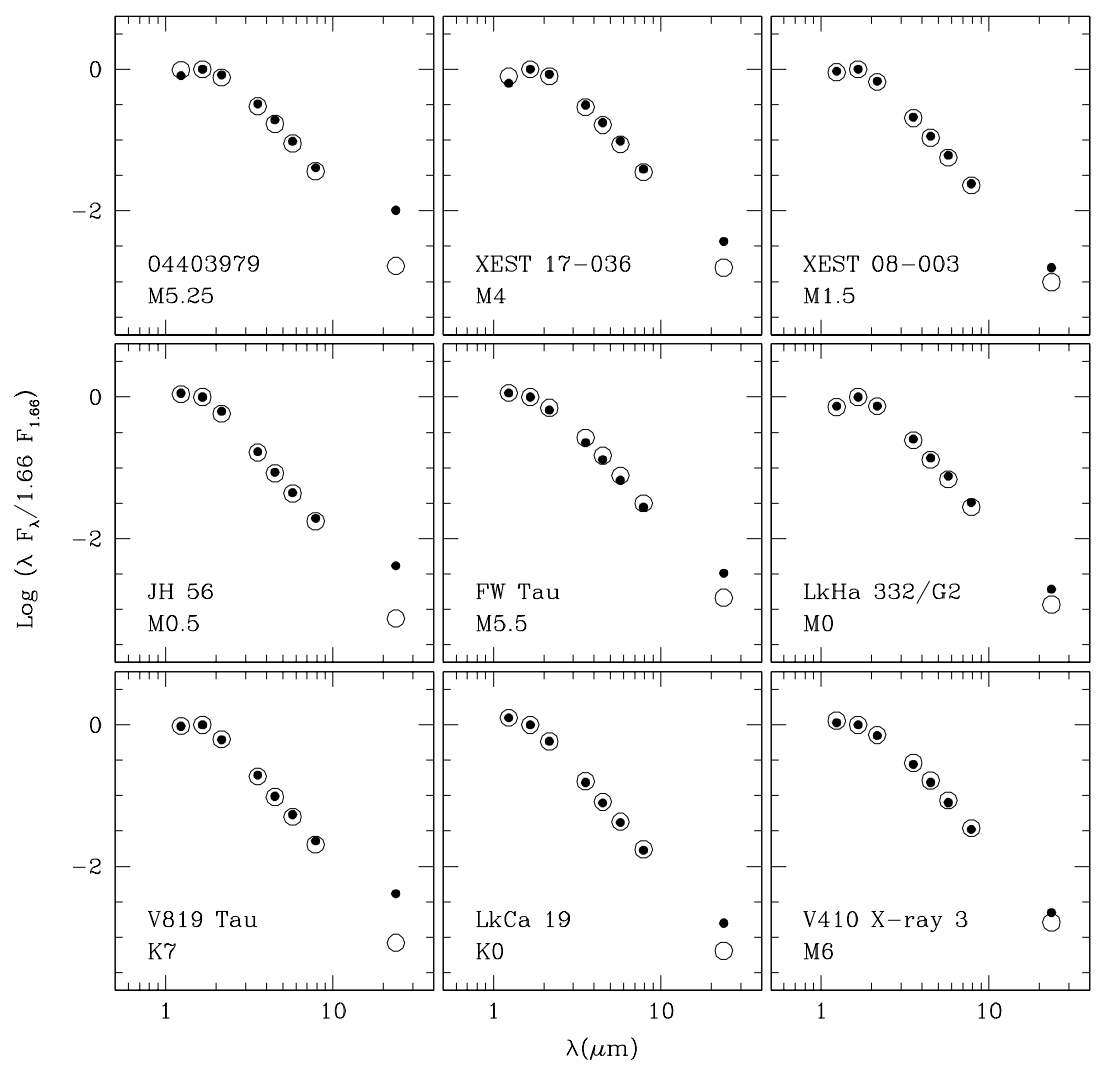

FIG. 17. - SEDs in Taurus that exhibit no excess emission at $\lambda<10 \mu \mathrm{m}$ and small excesses at $24 \mu \mathrm{m}$, which may indicate the presence of disks that have inner holes and are becoming optically thin at $24 \mu \mathrm{m}$ (evolved transitional disks) or disks that are composed of secondgeneration dust from collisions among planetesimals (debris disks). Each SED is compared to the SED of a stellar photosphere at the same spectral type (circles), which has been reddened by the extinction of the Taurus source and scaled to its $H$-band flux. 

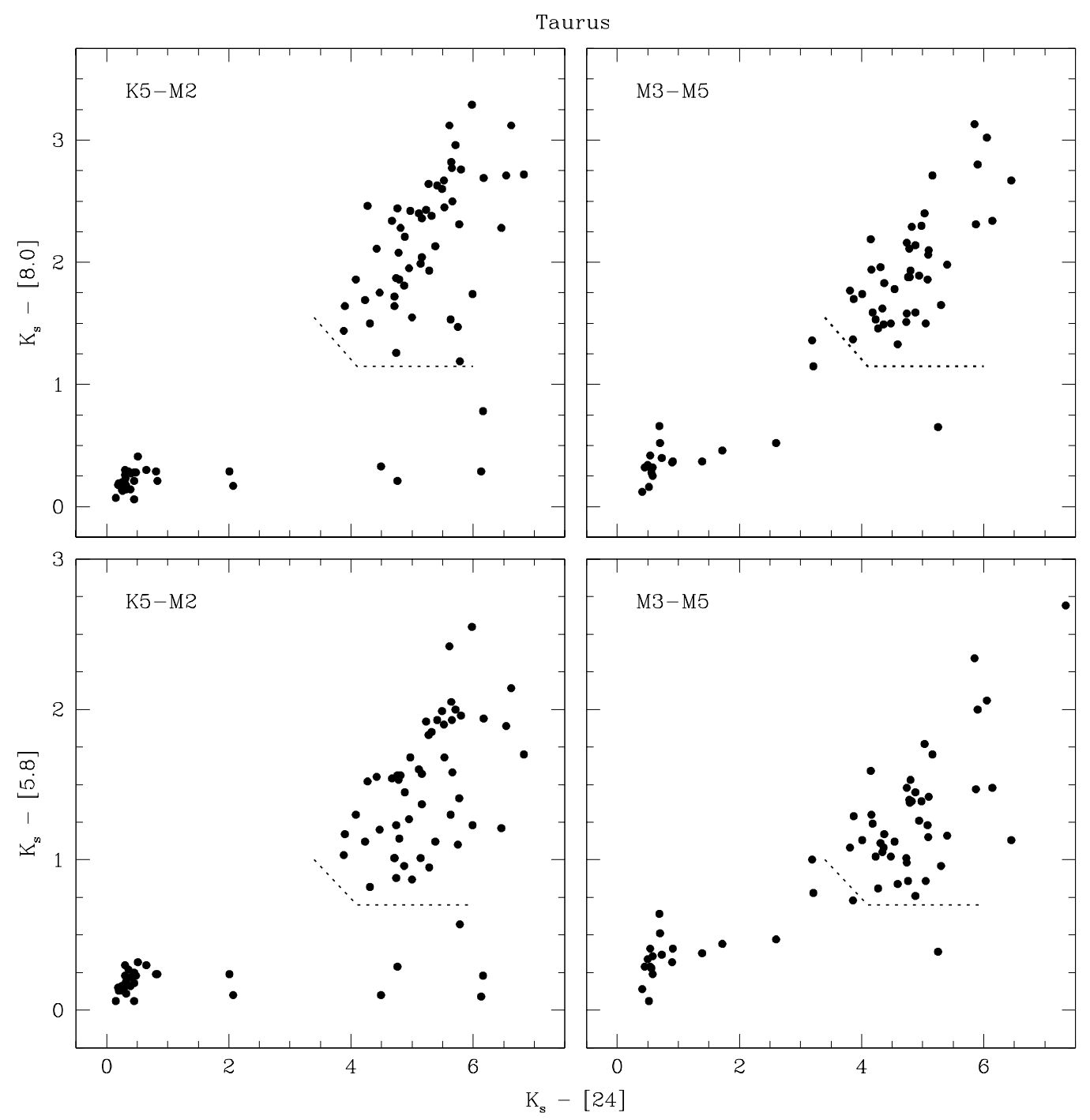

FIG. 18. - $K_{s}-[8.0]$ and $K_{s}-[5.8]$ versus $K_{s}-[24]$ for K5-M2 and M3-M5 members of Taurus $(\tau \sim 1$ Myr). We have marked the lower boundary of the primordial disks (dotted lines), which is plotted with data for other clusters in Figs. [19]25] 

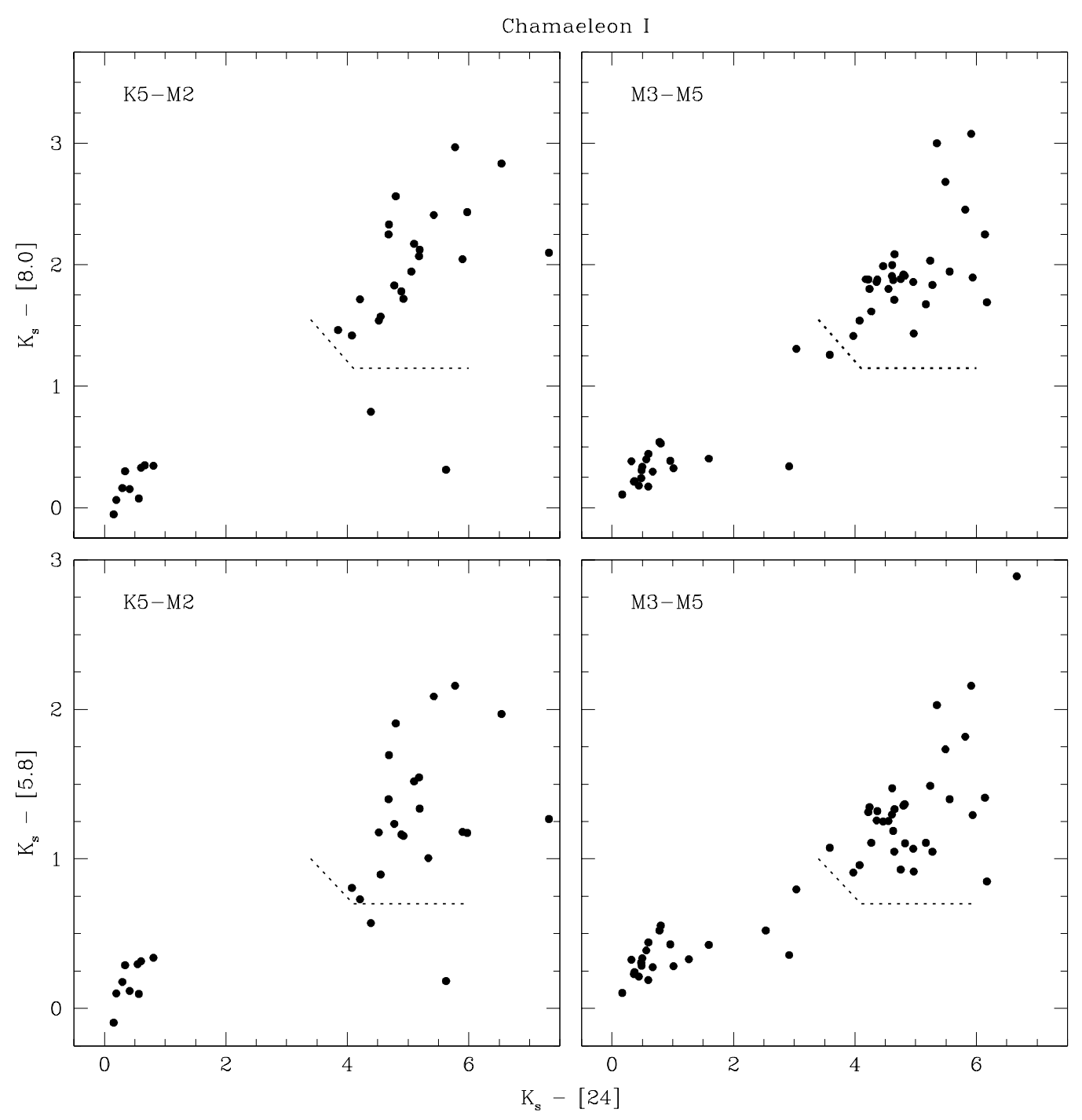

Fig. 19. $-K_{s}-[8.0]$ and $K_{s}-[5.8]$ versus $K_{s}-[24]$ for K5-M2 and M3-M5 members of Chamaeleon I $(\tau \sim 2-3$ Myr, Luhman et al. 2008a; Luhman \& Muench 2008). The lower boundary of the primordial disks in Taurus is indicated (dotted lines). 


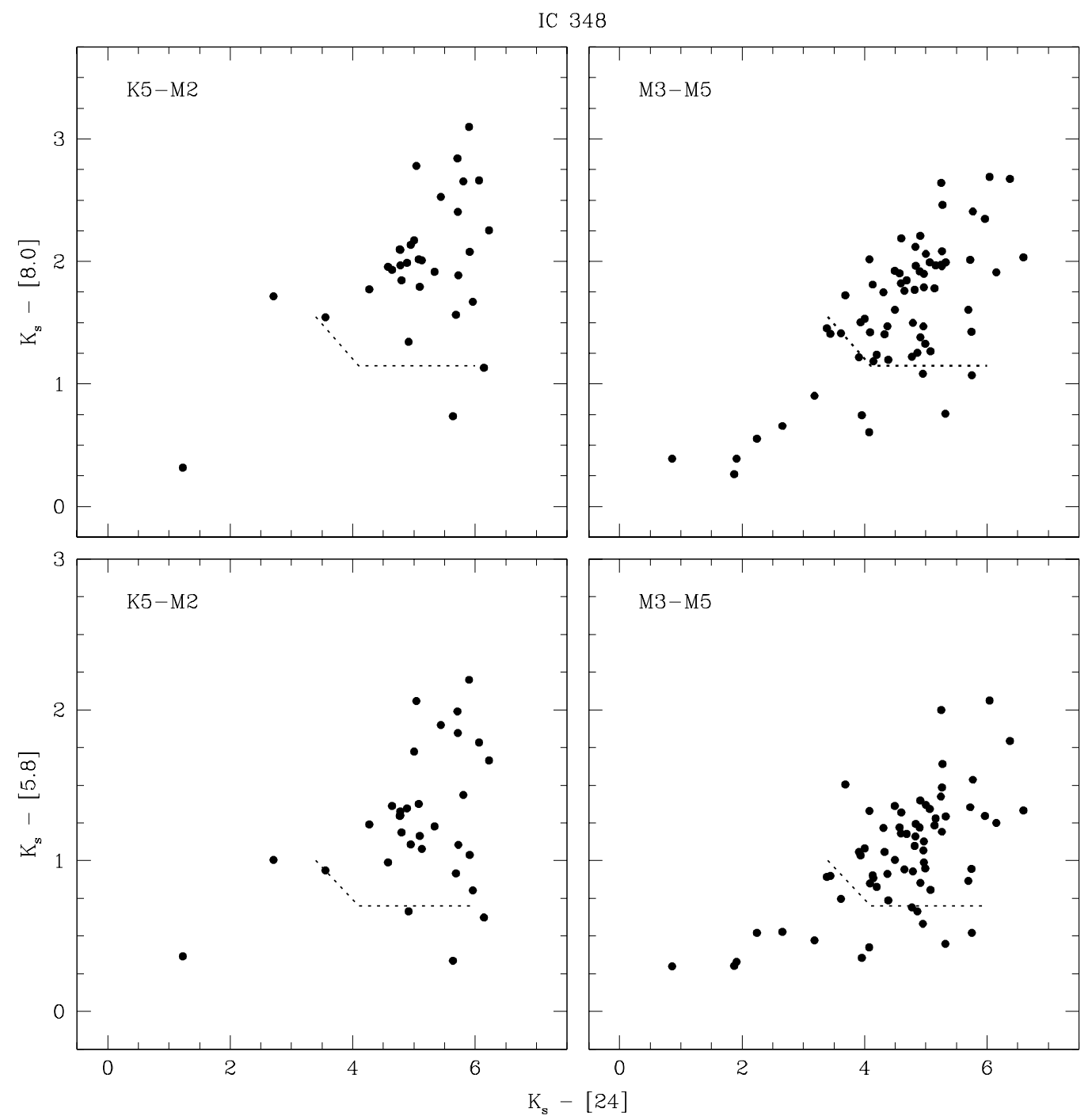

FIG. 20.- $K_{s}-[8.0]$ and $K_{s}-$ [5.8] versus $K_{s}-$ [24] for K5-M2 and M3-M5 members of IC $348(\tau \sim 2-3$ Myr, Lada et al. 2006; Muench et al. 2007). The lower boundary of the primordial disks in Taurus is indicated (dotted lines). 

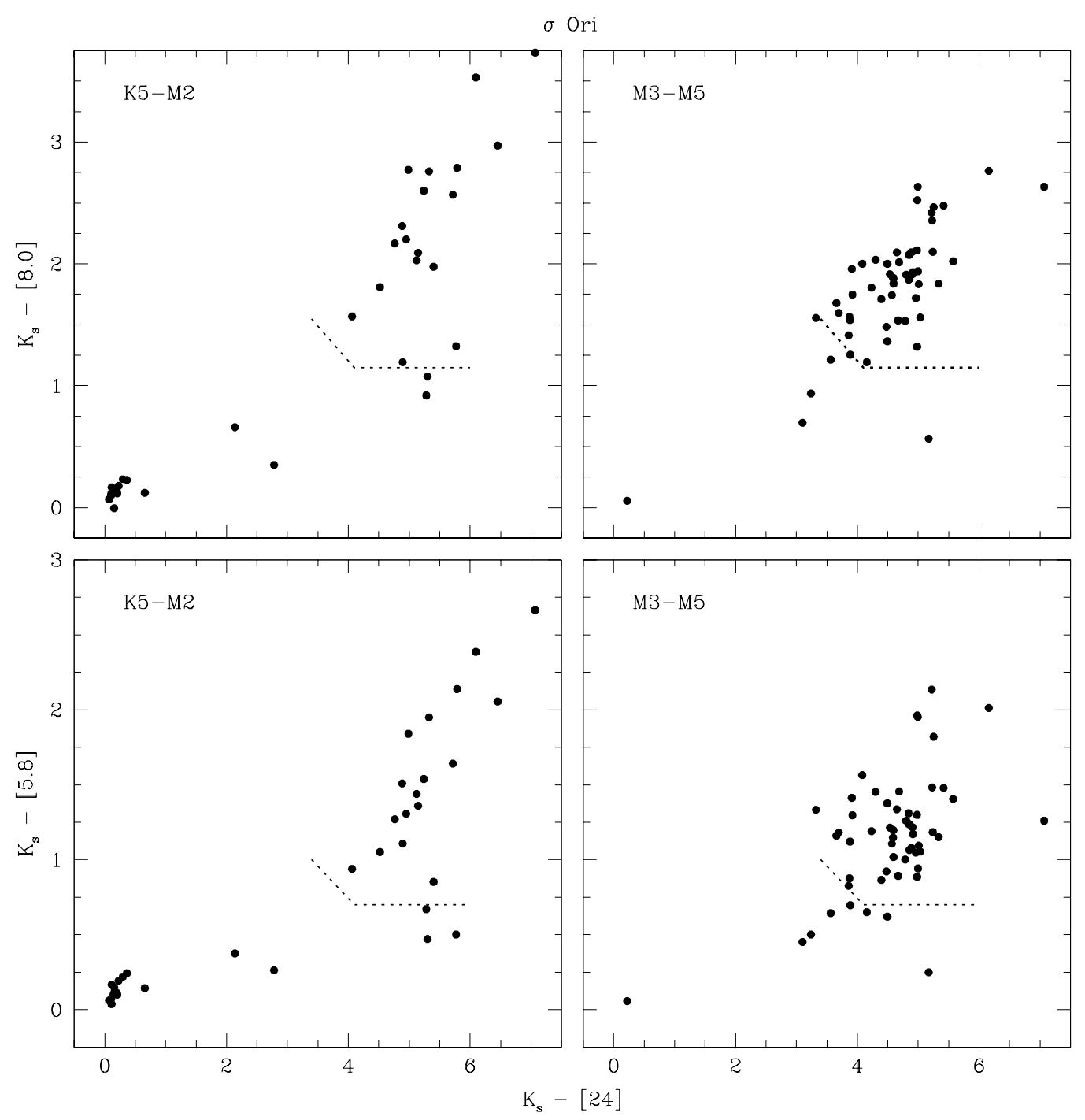

Fig. 21. $-K_{s}-[8.0]$ and $K_{s}-[5.8]$ versus $K_{s}-[24]$ for K5-M2 and M3-M5 members of $\sigma$ Ori $(\tau \sim 2-3$ Myr, Hernández et al. 2007a; Luhman et al. 2008b). The lower boundary of the primordial disks in Taurus is indicated (dotted lines). 
$\operatorname{Tr} 37$
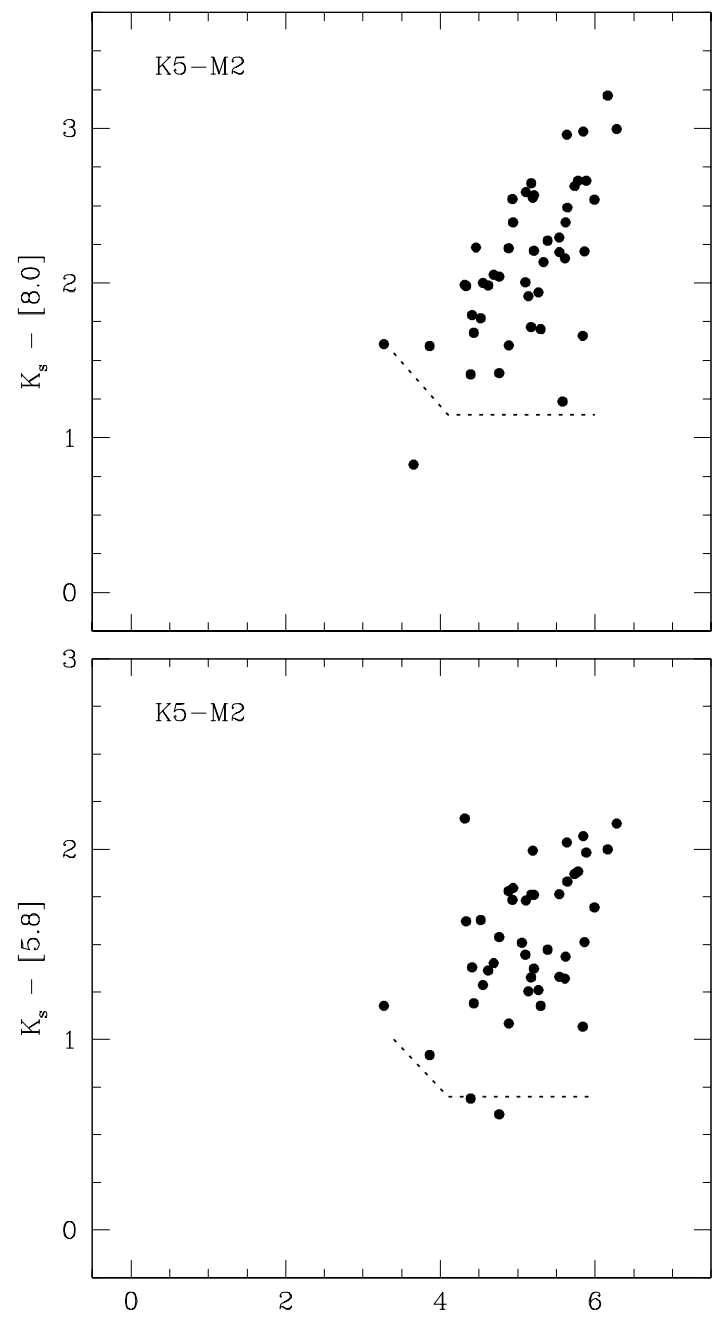

NGC 2362
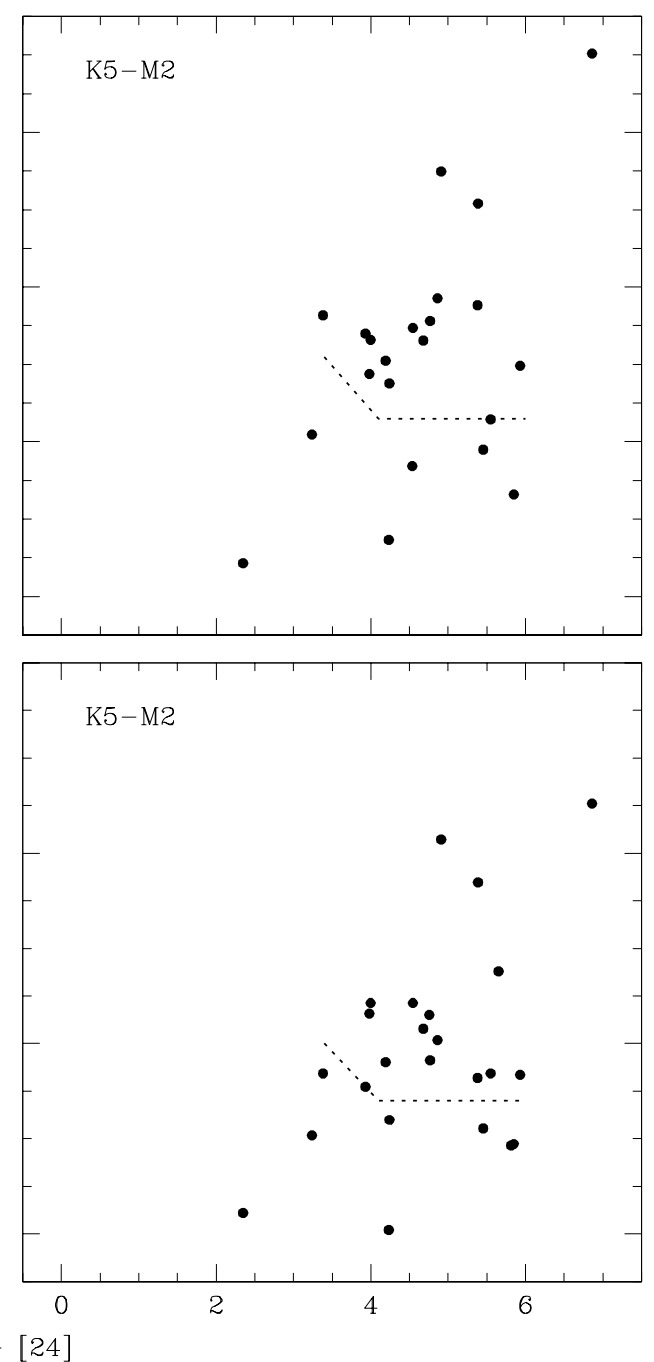

FIG. 22. $-K_{s}-[8.0]$ and $K_{s}-[5.8]$ versus $K_{s}-[24]$ for K5-M2 members of Tr 37 and NGC $2362(\tau \sim 4$ and 5 Myr, Sicilia-Aguilar et al. 2006; Dahm \& Hillenbrand 2007; Currie et al. 2009a). The lower boundary of the primordial disks in Taurus is indicated (dotted lines). 

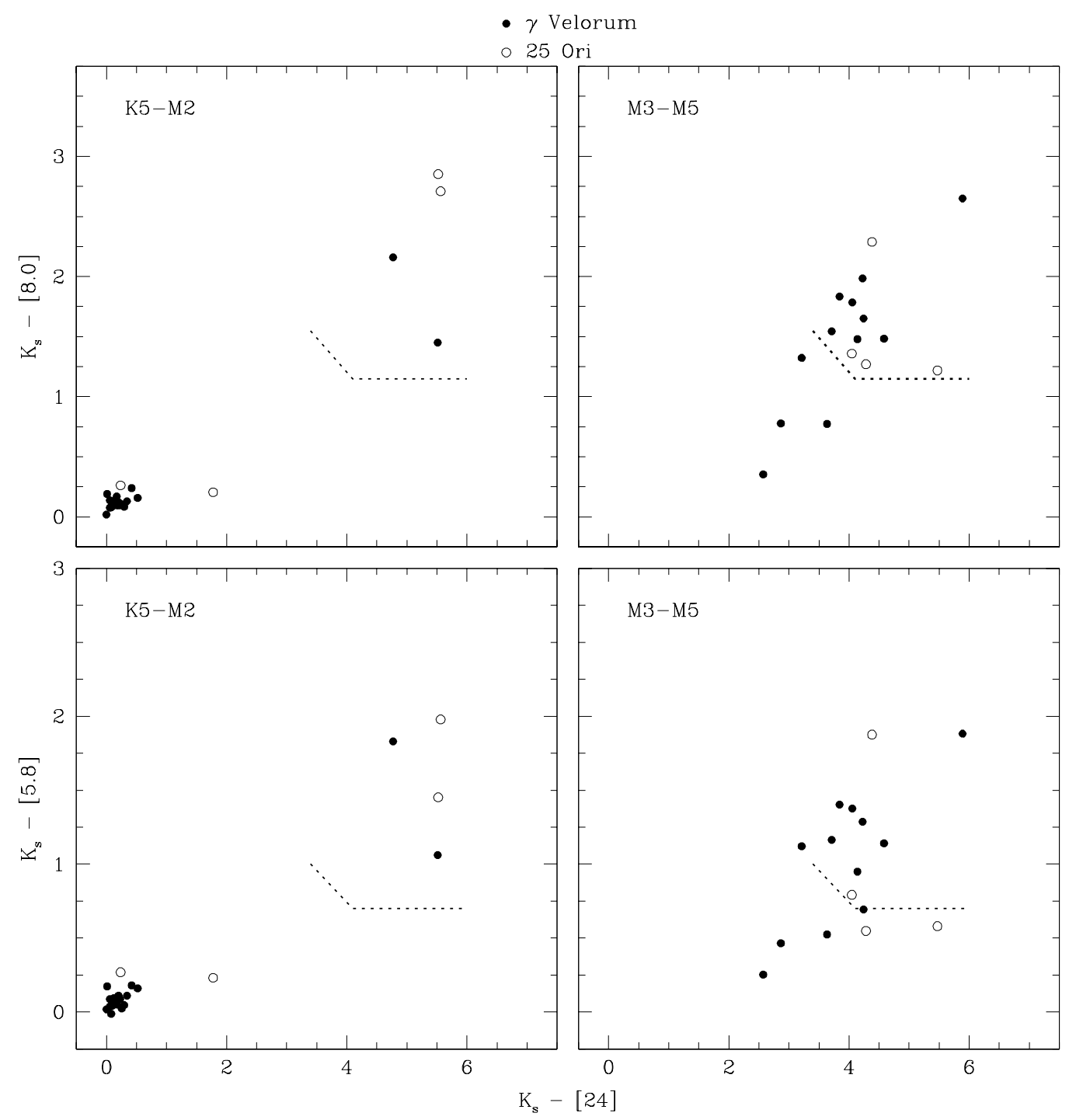

FIG. 23. - $K_{s}-[8.0]$ and $K_{s}-[5.8]$ versus $K_{s}-[24]$ for K5-M2 and M3-M5 members of $\gamma$ Velorum and 25 Ori $(\tau \sim 5$ and 10 Myr, Hernández et al. 2007b, 2008). The lower boundary of the primordial disks in Taurus is indicated (dotted lines). 

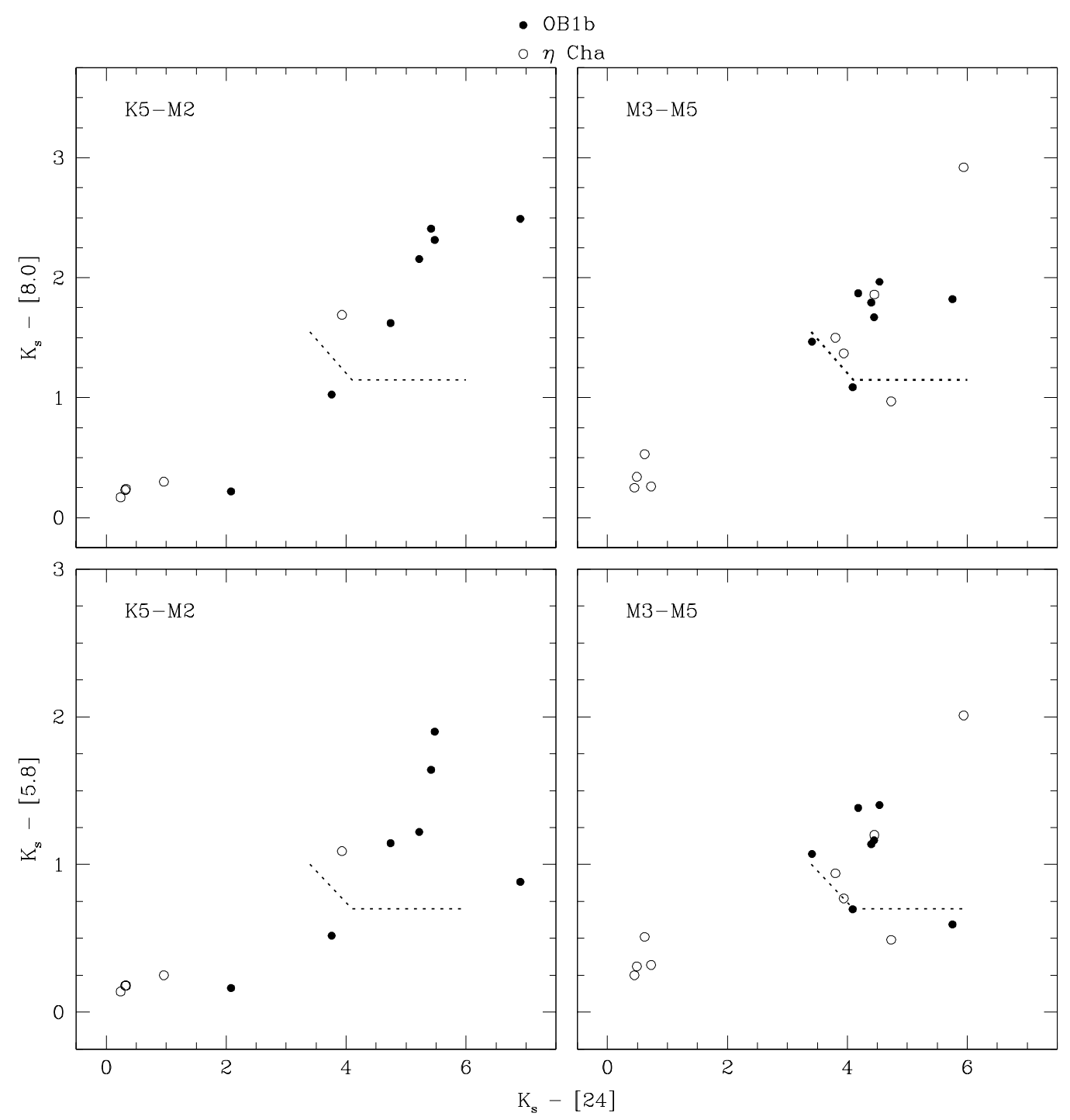

Fig. 24. $-K_{s}-[8.0]$ and $K_{s}-[5.8]$ versus $K_{s}-[24]$ for K5-M2 and M3-M5 members of Orion OB1b and $\eta$ Cha $(\tau \sim 5$ and 6 Myr, Megeath et al. 2005; Hernández et al. 2007b). The lower boundary of the primordial disks in Taurus is indicated (dotted lines). 


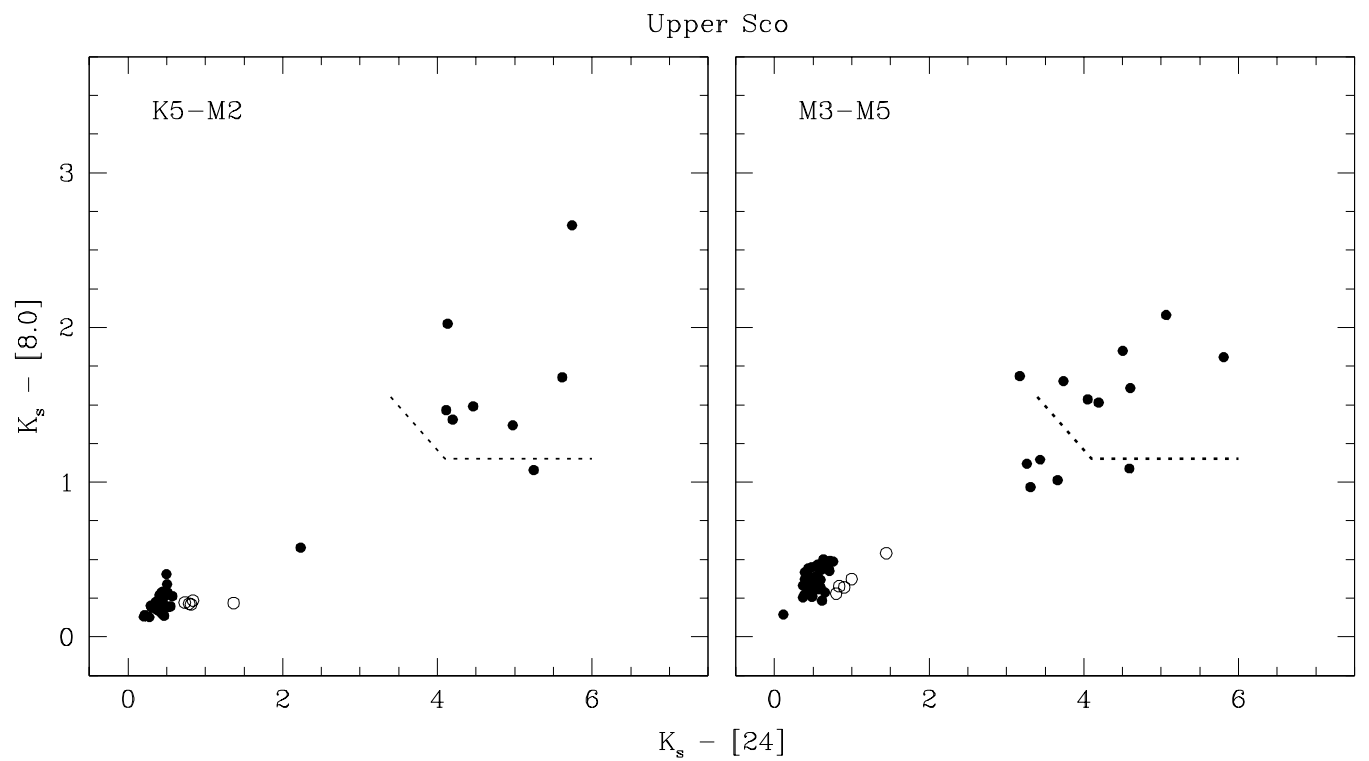

Fig. 25.- $K_{s}-[8.0]$ versus $K_{s}-[24]$ for K5-M2 and M3-M5 members of Upper Sco $(\tau \sim 5$ Myr, Carpenter et al. 2006, 2009). Candidate debris disks that were identified by Carpenter et al. (2009) are shown as open circles. The lower boundary of the primordial disks in Taurus is indicated (dotted lines). 


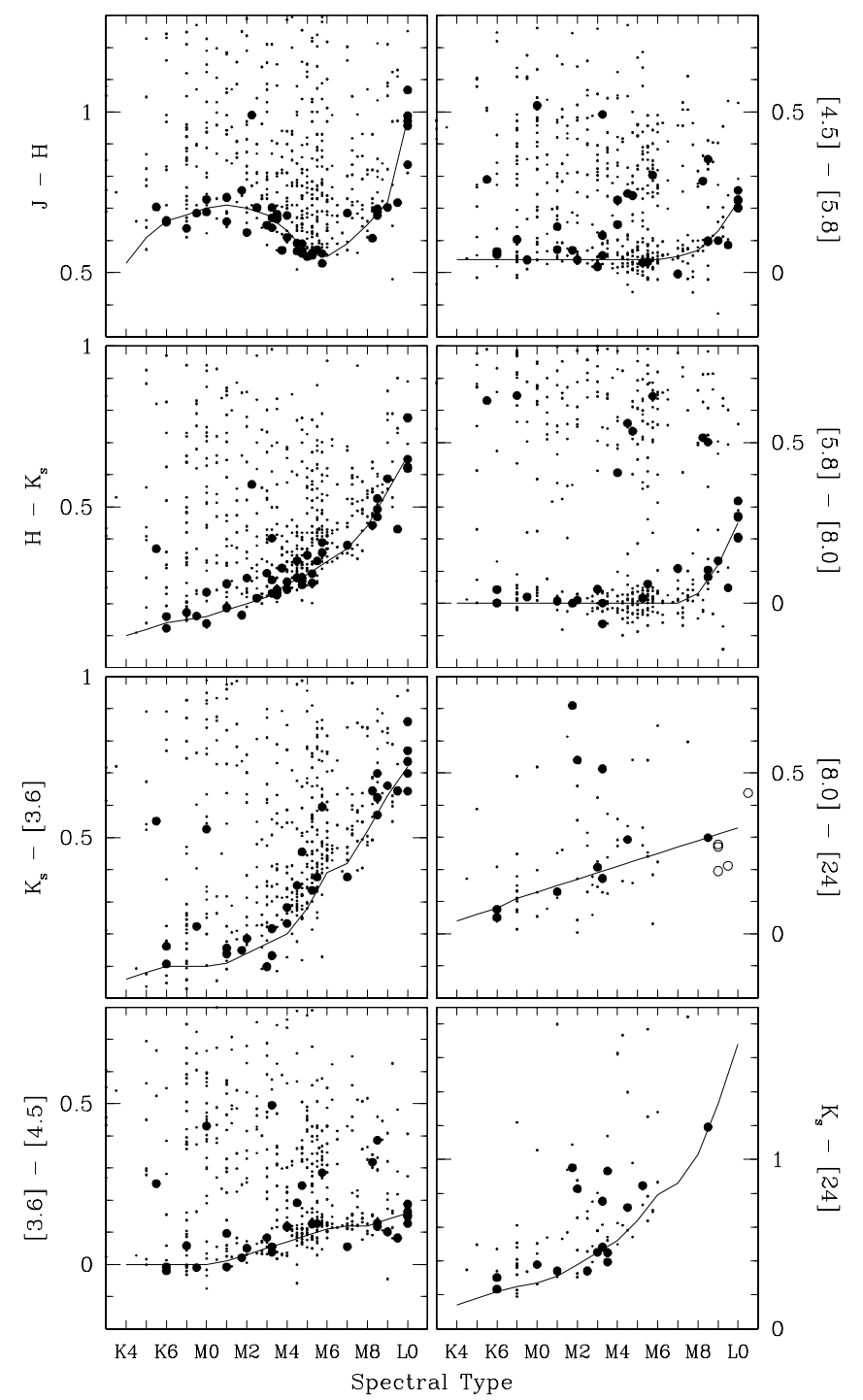

FIG. 26. - Infrared colors as a function of spectral type for members of the Taurus and Chamaeleon I star-forming regions (small filled circles) and young sources in the $\eta$ Cha, $\epsilon$ Cha, and TW Hya associations and in the solar neighborhood (large filled circles). The latter samples should have negligible extinction $\left(A_{V}<1\right)$. We have used these data to estimate the intrinsic colors of young stellar photospheres (solid lines, Table 13). Because few young late-type objects have been measured at $24 \mu \mathrm{m}$, we also show data for field dwarfs at M9-L0 in the diagram of $[8.0]-[24]$ colors (open circles). 